\title{
Psychosis and autism as diametrical disorders of the social brain
}

\author{
Bernard Crespi \\ Killam Research Professor, Department of Biosciences, Simon Fraser \\ University, Burnaby, BC V5A 1S6, Canada \\ crespi@sfu.ca http://www.sfu.ca/biology/faculty/crespi/ \\ Christopher Badcock \\ Department of Sociology, London School of Economics, London WC2A 2AE, \\ United Kingdom \\ C.Badcock@Ise.ac.uk \\ http://www.Ise.ac.uk/collections/sociology/whoswho/badcock.htm
}

\begin{abstract}
Autistic-spectrum conditions and psychotic-spectrum conditions (mainly schizophrenia, bipolar disorder, and major depression) represent two major suites of disorders of human cognition, affect, and behavior that involve altered development and function of the social brain. We describe evidence that a large set of phenotypic traits exhibit diametrically opposite phenotypes in autistic-spectrum versus psychotic-spectrum conditions, with a focus on schizophrenia. This suite of traits is inter-correlated, in that autism involves a general pattern of constrained overgrowth, whereas schizophrenia involves undergrowth. These disorders also exhibit diametric patterns for traits related to social brain development, including aspects of gaze, agency, social cognition, local versus global processing, language, and behavior. Social cognition is thus underdeveloped in autistic-spectrum conditions and hyper-developed on the psychotic spectrum.

We propose and evaluate a novel hypothesis that may help to explain these diametric phenotypes: that the development of these two sets of conditions is mediated in part by alterations of genomic imprinting. Evidence regarding the genetic, physiological, neurological, and psychological underpinnings of psychotic-spectrum conditions supports the hypothesis that the etiologies of these conditions involve biases towards increased relative effects from imprinted genes with maternal expression, which engender a general pattern of undergrowth. By contrast, autistic-spectrum conditions appear to involve increased relative bias towards effects of paternally expressed genes, which mediate overgrowth. This hypothesis provides a simple yet comprehensive theory, grounded in evolutionary biology and genetics, for understanding the causes and phenotypes of autistic-spectrum and psychotic-spectrum conditions.
\end{abstract}

Keywords: autism, cognition, genomic conflict, genomic imprinting, hyper-mentalism, psychosis, schizophrenia

People divide roughly, it seems to me, into two kinds, or rather a continuum is stretched between two extremes. There are people people and things people.

-W. D. Hamilton (2005, p. 205)

\section{Introduction}

We describe a new hypothesis that seeks to conceptually unify the analyses of psychosis and autism, two disorders of the human social brain (Burns 2004; 2006a; McAlonan et al. 2005). The core of this hypothesis is that psychosis and autism represent two extremes on a cognitive spectrum with normality at its center. Social cognition is thus underdeveloped in autism, but hyper-developed to dysfunction in psychosis. We also suggest that these forms of deviation from normal social brain development in either direction are mediated in part by alterations in developmental and metabolic systems affected by genomic imprinting, notably via effects of genes that are imprinted in the brain and in the placenta (Davies et al. 2005; Tycko \& Morison 2002). Genomic imprinting involves a developmental and physiological tug-of-war, in the growing fetus and child, between the effects of paternally expressed (maternally "imprinted," that is, maternally silenced)
Bernard Crespi, Professor of Evolutionary Biology at Simon Fraser University, has authored more than 100 publications and 4 books in the areas of social behavior, life-history evolution, and evolutionary genetics. He conducted his doctoral work with William Hamilton and Richard Alexander at the University of Michigan, followed by postdoctoral studies at Oxford and Cornell. Dr. Crespi has received the Theodosius Dobzhansky Prize and the E. O. Wilson Award from the Society for the Study of Evolution; and a Killam Fellowship from the Canada Council has allowed him to apply his expertise to the evolution of human cognition and psychiatric disorders.

Christopher BAdcock was born in 1946 and studied for his first degree and Ph.D. at the London School of Economics, where he is now a Reader in the Department of Sociology. Following a private didactic analysis with Anna Freud, he published a number of books and papers on Freud, evolutionary psychology, and genetics, and is now completing a book on the wider implications of the theory outlined in this paper. He is author of Evolutionary Psychology: A Critical Introduction (Polity Press, 2000), and currently teaches graduate and undergraduate courses on evolution and genetics. 
Crespi \& Badcock: Psychosis and autism as diametrical disorders of the social brain

genes, which favor enhanced growth as well as selfishness in interactions with the mother, and the effects of maternally expressed (paternally silenced) genes, which favor relatively constrained growth and other traits that tend to benefit mothers (Haig 2000b; 2004b). Relatively small genetic or epigenetic disruptions of this tug-of-war may increase the fitness of the child or mother, respectively, as in some disorders of placentation mediated by dysregulated imprinting (Haig 1999b; Oudejans et al. 2004); but larger alterations are pathological, and we hypothesize that they contribute to the development of either autistic-spectrum disorders (due to a paternal-gene bias) or psychotic-spectrum disorders (due to a maternal-gene bias) via their effects on growth, neurodevelopment, cognition, and behavior.

We unpack our hypothesis by first providing a brief background on the social brain, and how its development is altered in autism and psychosis. Second, we provide an overview of genomic imprinting and explain Haig's (2000b; 2004b) "conflict theory" for how imprinting has evolved. Third, we describe our nosological framework for conceptualizing autistic-spectrum conditions and what we call psychotic-spectrum conditions, and we explain how the conflict theory of imprinting provides an evolutionary basis for elucidating their genetic, epigenetic, and neurodevelopmental causes. Fourth, we describe how Prader-Willi syndrome and Angelman syndrome, which are caused by alterations of a region of chromosome 15 harboring imprinted genes, provide useful tests of the role of imprinted genes in autism and psychosis. Fifth, we contrast autistic-spectrum and psychotic-spectrum conditions for a wide range of anatomical, neurological, developmental, cognitive, behavioral, and epidemiological data. Our hypothesis predicts that to the degree that they represent opposite, generalized disorders, autistic- and psychoticspectrum conditions should exhibit diametric phenotypes for traits related to growth, development, and the social brain. Finally, we develop a conceptual model for how sex differences interact with genomic-imprinting effects, which can help to explain some key features of autisticand psychotic-spectrum epidemiology and symptoms.

We appreciate that autistic-spectrum and psychoticspectrum conditions are each highly heterogeneous, with myriad causes (Ronald et al. 2006; Ross et al. 2006a), and we are thus not proposing that these conditions are caused in any exclusive sense by alterations to genomic imprinting. We also stress that describing an evolutionary framework for understanding autism and psychosis does not in any way imply that these conditions should be considered as adaptive, even though the autistic and psychotic spectrums each involve a pattern of specialized cognitive strengths and impairments in relatively high-functioning individuals (Claridge 1997; Mottron et al. 2006). Our main goal instead is to integrate predictions from evolutionary theory and genetics with psychology, neuroscience, and psychiatry, to further our understanding of the major disorders of human cognition, affect, and behavior.

\section{The social brain}

A key initial insight into human evolution was the idea that the primary selective pressures shaping human cognitive development may be social rather than ecological (Emery 2000). This idea can be traced to Chance and Mead (1953), Jolly (1966), Humphrey (1976; 1983), Alexander (1989), and Brothers (1990), who have suggested that living in large, complex groups, under strong withingroup and between-group social competition for resources and mates, has selected for a "social brain," functionally designed by evolution mainly for solving social problems.

Recent studies have described how early development of components of the social brain is impaired in autism, which may lead to a cascade of social deficits, and how many of the core features of schizophrenia can also be understood in terms of dysregulation in multiple aspects of uniquely human social cognition (Arbib \& Mundhenk 2005; Baron-Cohen \& Belmonte 2005; Benes \& Berretta 2001; Burns 2004; 2006a). These advances have suggested that autism and schizophrenia are related to one another in some conceptual and etiological ways, because they both involve alterations in recently evolved human social behavior as central features (Burns 2006a). Although both disorders can be conceived as dimensional, grading more or less finely into normality (e.g., Frith \& Happé 2005; see also, Hill \& Frith 2003; Linney et al. 2003; Schürhoff et al. 2005), the relationship of autisticspectrum conditions with schizophrenia, and other conditions on the psychotic spectrum, has yet to be explicitly investigated in any detail. We do so here, in the context of evolutionary theory and genetics, with a focus on effects of genomic imprinting.

\section{Genomic imprinting}

Analysis of the social brain in contemporary neuroscience and psychology is yielding stunning insights into human cognition and psychiatric disorders. However, the conceptual social brain is not yet an evolutionary brain, because it has yet to fully incorporate central features of evolutionary biology, such as inclusive fitness theory (Foster et al. 2006; Hamilton 1964), intragenomic conflict (Burt \& Trivers 2006), social-behavioral ecology (e.g., Krebs \& Davies 1991), sex differences attributable to sex-differential selective pressures (e.g., Andersson 1994), and the genomic basis of recent human evolution (e.g., Crespi 2006; Voight et al. 2006). Each of these bodies of theory and data has important implications for understanding the evolution of the human social brain, its developmentalgenetic underpinnings, and its dysregulation.

Inclusive fitness theory forms a cornerstone of biology, in explaining how social interactions between genetically related individuals, such as mothers and offspring, have evolved (Alexander 1987; Hamilton 1964; Hrdy 1999). This theory predicts that under any degree of multiple paternity, genes subject to imprinting whose phenotypic effects lead to offspring extracting relatively high levels of limiting resources from mothers and other maternal kin are expected to be silenced in the maternal germ line (and thus paternally expressed in offspring) (Burt \& Trivers 2006; Haig 2000b; 2004b). In turn, these effects should be countered by selection for paternal silencing (and thus expression from only the maternally inherited chromosome) of genes whose phenotypic effects restrain the transfer of mother's resources, bringing maternal investment towards her own optimum level. This conflict theory 
for the evolution and patterns of genomic imprinting has been supported by a large body of evidence on the functions and expression patterns of imprinted genes (Cattanach et al. 2004; Haig 1996; 1999b; 2004a; 2004b; McMinn et al. 2006; Plagge et al. 2004; Smith et al. 2006), and it provides a robust theoretical framework for analyzing the roles of imprinted genes in human development and evolution. Although imprinted genes comprise only about $1 \%$ of the genome, they are disproportionately involved in growth, especially with regard to placental and brain development and function (Tycko \& Morison 2002); they are highly pleiotropic in their effects; and they can be dysregulated in more ways than non-imprinted genes. Thus, imprinted-gene expression can be affected by alterations in nucleotide sequence, by epigenetic variation (such as methylation and histone modification), by "imprinter" genes that regulate application, maintenance, and removal of imprints (Wilkins 2005), and by environmentally induced effects on imprinted gene expression (Dolinoy et al. 2006).

Most studies of genomic imprinting have focused on genes expressed during prenatal and neonatal development, where conflict is manifested in aspects of maternal-fetal interactions during placentation and neonatal growth (Angiolini et al. 2006; Crespi \& Semeniuk 2004; Haig 1993; 1996; 2004a; 2004b). The placenta has evolved as a focal point for genomic conflict due to its function in the transfer of resources between mutually dependent individuals that bear genes with partially divergent inclusive fitness interests (Coan et al. 2005; Haig 1993; 1996). Many of the common disorders of pregnancy, including gestational diabetes, pre-eclampsia, and fetal growth restriction, arise in part from breakdowns in the dynamically balanced, "tugof-war" nature of physiological systems subject to maternal-fetal conflict and imprinting effects (Cattanach et al. 2004; Haig 1993; 1996; 1999b; McMinn et al. 2006; Oudejans et al. 2004; Reik et al. 2003).

A considerable proportion of known imprinted genes are expressed exclusively or predominantly in the brain, where they influence aspects of behavior (Curley et al. 2004; Davies et al. 2001; 2005; 2006; Isles et al. 2006; Keverne 2001a; 2001b). The brain can be conceived as analogous to the placenta in that both organs mediate the transfer of fitness-limiting resources in networks of kin (Badcock \& Crespi 2006). As in the case of placentation, disruption in systems involving brain-expressed imprinted genes can lead to major neurological and physiological disorders (Badcock 2000; Davies et al. 2001; Haig \& Wharton 2003; Isles et al. 2006). Developmental systems regulated by imprinting effects are unusual in that they can be disrupted in two diametrically opposed ways, towards either paternalgene or maternal-gene bias. Disorders affected by imprinting should thus exhibit diametric phenotypes, as seen clearly, for example, in Beckwith-Wiedemann syndrome involving overgrowth versus the Silver-Russell undergrowth syndrome (Cerrato et al. 2005; Eggermann et al. 2005; 2006). We propose that such diametric effects extend to brain and behavior, and these effects help to account for some of the major features of human cognitive architecture.

\section{The imprinted brain}

In 2002, Badcock proposed that insights from autism research suggest that we have evolved two parallel cognitive systems, which he termed mentalistic and mechanistic cognition (see Badcock 2004). Mentalistic cognition (or simply mentalism, otherwise know as theory of mind, folk psychology, or mentalizing) evolved for interaction with other people in a psychological environment, whereas mechanistic cognition (folk physics) evolved in parallel for interaction with the physical environment (for a comparable view, see Kuhlmeier et al. 2004). Badcock also proposed that if, as is generally accepted, many symptoms of autism can be seen in terms of deficits in functions such as gazemonitoring, intentionality, shared attention, and theory of mind in general (Baron-Cohen 1995), then some common symptoms of paranoid schizophrenia, such as delusions of being watched or spied on, erotomania or delusions of persecution, conspiratorial delusions, and religious and magical delusions, could be seen as pathologically hypertrophied equivalents, or in general terms as hyper-mentalism (Badcock 2004; see also Abu-Akel 1999; Abu-Akel \& Bailey 2000 for supporting views). Badcock and Crespi (2006) suggested that evolutionary and genetic foundations of autism might be found in some combination of enhanced expression of paternally active genes and reduced expression of maternally active ones in brain development and behavior (Badcock \& Crespi 2006). Here, we extend these basic insights by showing how the two extremes of the mechanistic-mentalistic continuum - what we call autistic- and psychotic-spectrum conditions - can be represented as diametric opposites for a large suite of phenotypic traits, with the diametric nature understood in terms of the two possible directions, paternal and maternal, for imbalances in imprinted gene effects. The cognitive and behavioral effects of such imbalances are most clear for known syndromes mediated by imprinting effects, but can, we contend, be generalized and extended to major disorders of the social brain.

\subsection{Autistic-spectrum conditions}

Autism is a spectrum of conditions, all of which involve some combination of impairments in social interaction; language and communication; and repetitive, restricted behaviors or interests (Happé et al. 2006). This spectrum includes Kanner (infantile) autism, Asperger syndrome, and a set of other conditions including Rett syndrome (LaSalle et al. 2005), Fragile X syndrome (Belmonte \& Bourgeron 2006), and Turner syndrome (Skuse 2005), all of which involve autistic features in a substantial proportion of affected individuals. Conti-Ramsden et al. (2006), Herbert and Kenet (2007), and Smith (2007) also describe close links between autism and Specific Language Impairment, and obsessive-compulsive disorder (OCD) may exhibit a closer association with the autistic spectrum than with the psychotic spectrum (Abramson et al. 2005; Bejerot 2007; Bürgy 2007; Fineberg et al. 2007). We have therefore conceptualized the autistic spectrum in terms of its three main criteria and their main component phenotypes (Fig. 1), showing that these criteria partially overlap in their phenotypic expression, and by implication in their genetic underpinnings (Happé et al. 2006). By our hypothesis for the phenotypic structure of autistic conditions, at the core of these features we find a reduction in mentalistic cognition, affect, and behavior - a relatively underdeveloped social brain. 
Crespi \& Badcock: Psychosis and autism as diametrical disorders of the social brain

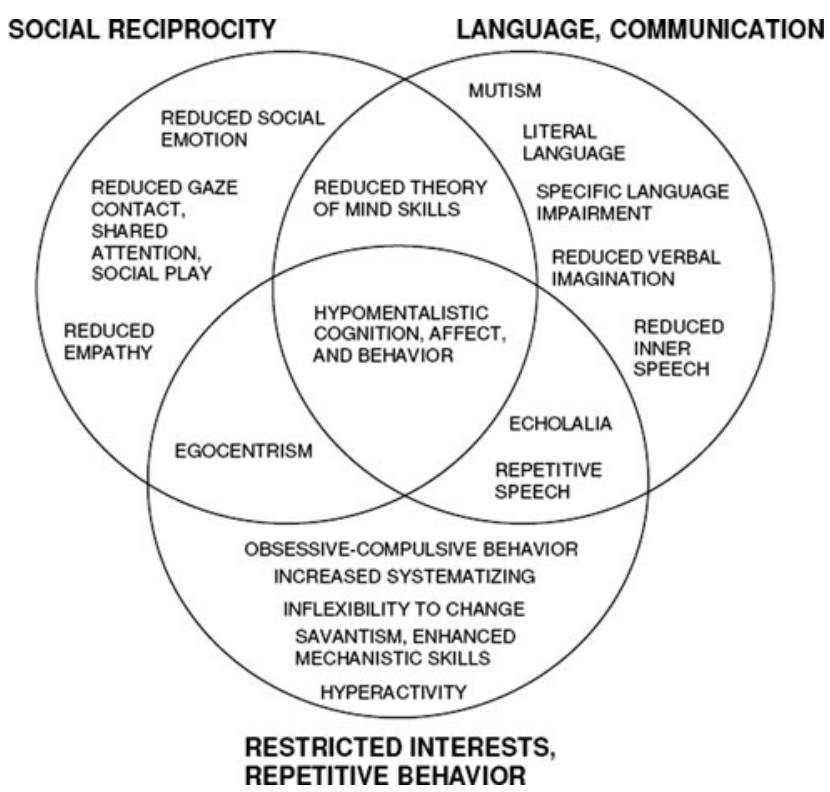

Figure 1. The autistic spectrum can be visualized in terms of three suites of traits that partially overlap in their phenotypic expression and genetic underpinnings, with each suite of traits grading more or less smoothly into each other and into normality. At the core of the autistic spectrum we find a reduction in mentalistic cognition, affect, and behavior, which can be mediated by effects on the development of social reciprocity, language and communication, and restrictive interests and activities, or by some combination of effects from these three domains. Recent studies suggest that the degree of genetic and phenotypic overlap between these three domains of the autistic spectrum appears similar in magnitude to the overlap between the three main conditions characterizing the psychotic spectrum, which are shown in Figure 2.

Previous theory for understanding the evolutionary and developmental bases of autism has focused on sex differences and how they relate to autistic phenotypes. Asperger (1991) thus suggested that "the autistic personality is an extreme variant of male intelligence," and Baron-Cohen (2002; 2003) has developed this idea into an "extreme male brain" theory of autism, which posits that the primary differences between autistic and normal cognition parallel the differences between the sexes. By this theory, autism can be "explained psychologically by an impaired capacity for empathizing, or modeling the mental states governing the behavior of people, along with a superior capacity for systemizing, or inferring the rules governing the behavior of objects" (Baron-Cohen \& Belmonte 2005, p. 109; see also Baron-Cohen 2002; Baron-Cohen et al. 2005). This hypothesis is consistent with a large body of evidence, including (1) a male-biased sex ratio in autism; (2) enhanced empathy, better ability to detect emotions, and faster language development in girls, whereas boys show increased ability and interests in activities related to systemizing (Baron-Cohen 2002; BaronCohen et al. 2005; McClure et al. 2004); (3) links between higher prenatal exposure to testosterone and autistic traits (Knickmeyer et al. 2005; Lutchmaya et al. 2002a; 2002b); and (4) higher scores for males on a test characterizing individuals along an autistic spectrum (Baron-Cohen et al. 2001; 2005).
Badcock and Crespi (2006) have described genetic, neurological, and behavioral evidence relevant to the hypothesis that important aspects of autism may represent not the extreme male brain per se, but rather the extreme paternally biased imprinted brain. Thus, autism disproportionately involves imbalances in development that lead to increased effects of paternally expressed genes at loci subject to imprinting, relative to maternally expressed ones. Such paternally expressed genes are expected to drive development and cognition towards a more resource-demanding phenotype, similar to a phenotype generally more characteristic of males than females (Badcock \& Crespi 2006). The imprinted brain theory for autism is consistent with Baron-Cohen's body of evidence, but it can also help explain other key features of autism, such as the much more male-biased sex ratio in Asperger syndrome and high-functioning autism than in severe, Kanner autism (Folstein \& Rosen-Sheidley 2001), and the observation that many factors other than sex and fetal testosterone are involved. Evidence for epigenetic dysregulation of imprinted genes in autism is also reviewed by Schanen (2006).

Badcock and Crespi (2006) also describe how some central aspects of the autistic spectrum may be explained by their hypothesis. Thus, extreme deficits in the socalled maternal brain (mainly the highly developed neocortex) (Keverne 2001a) but more or less normal function of the paternal brain (mainly the limbic system), may lead to the loss of language, mental retardation, and repetitive behavior typical of infantile (Kanner) autism, whereas increased paternal-brain effects, but relatively spared maternal-brain function, may lead to high-functioning autism or Asperger syndrome, which involves specific deficits in social cooperation and reciprocity (Badcock \& Crespi 2006; Constantino \& Todd 2005; Fitzgerald 2004, pp. 30-41; Rinehart et al. 2002a). In both cases, autism results in part from disrupted tension between neurodevelopmental and physiological agents of intragenomic conflict. As for imprinted gene effects in placental disorders and carcinogenesis (e.g., Angiolini et al. 2006; Lee 2003; McMinn et al. 2006), the resulting phenotype is more or less pathological, but the nature of the deviation from normality provides insight into its underlying genomic, physiological, and evolutionary causes. The main phenotypic feature of autism that may reflect the conflict theory of genomic imprinting is that autism involves increased "self-oriented" and indeed "selfish" behavior, expressed most clearly as deficits of cooperative social behavior and augmentation of mechanistic cognition (Badcock 2004; Badcock \& Crespi 2006). We use the term mechanistic (rather than systemizing) cognition because mechanistic refers more generally to the physical world, including aspects of sensation; cause-effect inference; mechanistic relationships of child with mother (Kanner 1949); and bottom-up, non-abstract, less centrally coherent processing of information (Vermeulen 2001, p. 28).

\subsection{Psychotic-spectrum conditions}

Psychosis is literally a disordering of the psyche, the Greek "soul." In schizophrenia, such disordering commonly involves delusions and auditory hallucinations, loss of coherence and logic in thought and discourse, and 
emotionality (affect) externally reduced or inappropriate to social context (Tamminga \& Holcomb 2005). Auditory hallucinations, a primary symptom found in over $60 \%$ of persons diagnosed with schizophrenia, are also common in persons with bipolar disorder or major depression (Baethge et al. 2005; Kempf et al. 2005; 2007; Tsuang et al. 2004), as well as in non-clinical settings (Bentall 2003a). Bipolar disorder and major depression often involve other psychotic symptoms such as delusions, as well as symptoms related to dysregulated emotionality (Boks et al. 2007b). Schizophrenia, bipolar disorder, and major depression thus exhibit broad phenotypic overlap, as shown in Figure 2; and they also partially overlap in their polygenic underpinnings (Blackwood et al. 2007; Craddock \& Forty 2006; Potash 2006; Van Den Bogaert et al. 2006). These so-called psychotic-spectrum conditions also include schizotypy (Claridge 1997), Klinefelter syndrome (Boks et al. 2007a), velocardiofacial syndrome (Feinstein et al. 2002), and dyslexia (Condray 2005), all of which exhibit a notably elevated incidence of schizophrenia or affective psychosis, or a suite of physiological and neurological phenotypes characteristic of these conditions. We have conceptualized schizophrenia, bipolar disorder, and major depression as exhibiting partial overlap in their phenotypic features, with psychosis and hyper-mentalistic cognition, affect, and behavior at their core (Fig. 2).

Like autism, schizophrenia, bipolar disorder, and major depression each grades more or less smoothly from disorder into normality (Claridge 1997; Happé et al. 2006).

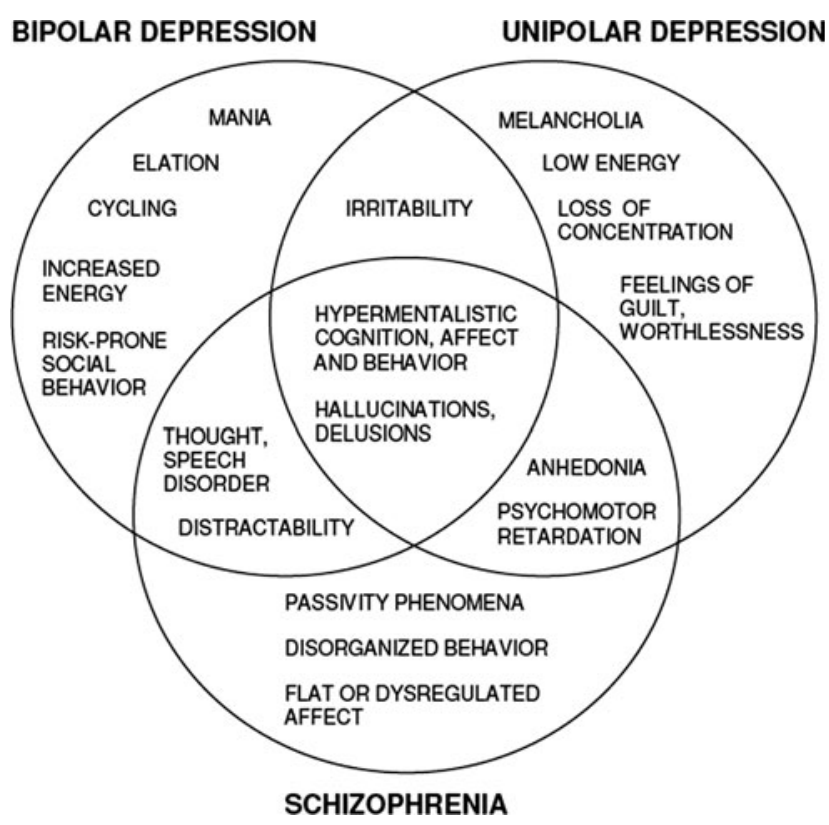

Figure 2. The psychotic spectrum can be visualized in terms of three main conditions - schizophrenia, bipolar disorder, and major depression - that grade into one another and exhibit partial overlap in their phenotypic expression and genetic underpinnings. These three conditions have historically been considered as largely separate, but recent genetic studies, and consideration of intermediate conditions, have demonstrated that they share a broad range of features and risk factors. At the core of the three conditions we find hyper-development in aspects of mentalistic cognition, affect and behavior, especially psychotic symptoms such as hallucinations and delusions.
Each of these conditions also exhibits a strong genetic component to its expression, but with many genes involved and different combinations of these genes underlying the phenotypes involved (Rapoport et al. 2005; Tamminga \& Holcomb 2005). Psychosis in schizophrenia, bipolar disorder, major depression, and schizotypy involves socalled positive, first-rank symptoms, which mainly include magical ideation, delusions, hallucinations, paranoia, thought disorder, and referential thinking. Such positive symptoms comprise a much higher proportion of the genetic liability to schizophrenia and schizotypy than do negative symptoms (Kremen et al. 1998; Vollema et al. 2002; Yaralian et al. 2000), and positive and negative symptoms appear to be independently heritable to a considerable degree (Fanous et al. 2001; Linney et al. 2003).

A logical consequence of the imprinted-brain hypothesis for the etiology of autism is that the converse disruption, towards stronger relative effects of maternally expressed imprinted genes, should also involve altered growth, development, and cognition. We describe evidence here that this direction of disrupted imprinting represents a contributing cause in the development of psychotic-spectrum conditions. By contrast with autism, imbalances towards increased effects of maternally expressed imprinted genes, or reduced effects from paternally expressed imprinted genes, should engender changes in physiology, morphology, and behavior that can be construed as more or less pathological manifestations of effects that are normally beneficial to mothers and other maternal relatives (Haig 2000a; 2000b; 2003; 2004b).

Our hypothesis is focused primarily on explaining phenotypes involved in psychosis, as these represent central traits exhibited in schizophrenia, schizotypy, bipolar disorder, and major depression (Fig. 2) (Crow 2004a; 2004b; 2004c; Keverne 1999). Negative symptoms such as social withdrawal, perseveration, apathy, and flat affect - as seen mainly in "deficit" schizophrenia - apparently involve a relatively large element of major neurophysiological pathology (such as grey matter loss) as well as altered function (e.g., Chua et al. 1997; Frith 1992). Such symptoms have been used as evidence for "autism" or "autistic traits" in schizophrenia, velocardiofacial syndrome, and Prader-Willi syndrome (Frith \& Frith 1991; Nylander \& Gillberg 2001; Sheitman et al. 2004; Veltman et al. 2004; 2005; Vorstman et al. 2006), but in each case these inferences of similarity have been based entirely on observation or data from questionnaires, interviews, scales, or checklists. By contrast, biological criteria, including neuroanatomy, neurophysiology, and genetics, demonstrate notable similarities of velocardiofacial syndrome, Klinefelter syndrome and Prader-Willi syndrome with disorders on the psychotic spectrum, especially schizophrenia (e.g., DeLisi et al. 2005; Eliez 2007; Eliez \& van Amelsvoort 2005; Holsen \& Thompson 2004; Lee et al. 2005).

The most useful information for evaluating our hypothesis comes from the relatively non-pathological points on the salient cognitive spectra: For autism, this is Asperger syndrome, high-functioning autism, and non-clinical individuals with autistic traits; and for psychosis, this is manifested most clearly in "healthy schizotypy" (Claridge 1997). However, we will consider all traits and conditions on the psychotic spectrum as potentially amenable to some degree of falsifiable explication by our hypothesis. Thus, 
Crespi \& Badcock: Psychosis and autism as diametrical disorders of the social brain

by analogy with hypothesized Kevernian maternal-brain and paternal-brain effects in autistic conditions (Badcock \& Crespi 2006), negative symptoms of schizophrenia and depression such as anhedonia, loss of will, flat affect, and psychomotor retardation may be associated with relatively decreased paternal-brain influences and a maternal brain that is either relatively unaffected, or that sends hypermentalistic outputs to the limbic system (e.g., paranoia eliciting fear, or feelings of guilt imposing anhedonia). In comparison, positive symptoms appear to be more a consequence of increased maternal-brain influences on cognition and behavior, with the paternal brain relatively unaltered.

As autism involves traits characteristic of an "extreme male brain" (Baron-Cohen et al. 2005), we predict that, in comparison, psychotic-spectrum disorders should reflect neuroanatomy, cognition, and behavior that are relatively more characteristic of females. We stress that the male-female axis, and the phenotypic axis defined by effects of paternally versus maternally expressed imprinted genes, are not the same: Both sexes exhibit effects from brain-expressed imprinted genes, and sex differences are driven by selection in diverse contexts. But the axes overlap; they may share mechanisms of development, and, as described later, the way that these axes interact may help to explain sex biases in the incidence and some major features of autistic- and psychotic-spectrum conditions.

\section{Prader-Willi and Angelman syndromes}

Prader-Willi and Angelman syndromes result from opposite disruptions (usually deletions or duplications) of a suite of imprinted genes on chromosome 15. PraderWilli syndrome is caused by the downstream developmental effects of imbalance towards increased relative expression of maternal genes in this region, and Angelman syndrome is due to imbalance towards less maternal gene expression (Bittel \& Butler 2005; Dan \& Boyd 2003; Whittington et al. 2004; Yamasaki et al. 2003). Both syndromes have major impacts on cognition, behavior, and psychopathology, and as a result, they provide useful tests of our hypothesis. If our hypothesis is correct, then Prader-Willi syndrome should involve increased rates of psychosis, and Angelman syndrome should involve a high incidence of autism. The power of such predictions is tempered primarily by the large magnitude of the perturbations that cause these syndromes: Reducing levels of imprinted gene expression to zero or doubling them (Bittel \& Butler 2005) probably leads to any number of purely pathological effects that may not be clearly indicative of the nature of the disrupted adaptive systems.

The phenotype of Prader-Willi syndrome can be divided into two main life-history stages. Prior to the usual age of weaning, this syndrome involves lack of appetite, poor suckling ability, a weak cry, inactivity, and sleepiness; by contrast, after this age, it involves extreme and unselective overeating (Dykens et al. 2000; Holland et al. 2003; Whittington \& Holland 2004). Haig and Wharton (2003) have suggested that these features of Prader-Willi syndrome reflect an extreme manifestations of traits that benefit the mother by making the baby less demanding on her resources, both before weaning (when food intake and energetic demands are reduced) and after weaning (when ingestion of any solid food available may ease provisioning). Prader-Willi syndrome also involves low birth weight and growth hormone deficiency (GillessenKaesbach et al. 1995; Goldstone 2004), which are consistent with increased relative developmental effects from maternally expressed imprinted genes.

Prader-Willi syndrome engenders a very high incidence of psychosis in adulthood (Verhoeven et al. 2003; Vogels et al. 2003; 2004). Such psychosis is found predominantly in cases of maternal uniparental disomy (UPD) (with 61\% of individuals exhibiting symptoms) compared to deletion (17\%) (Soni et al. 2007). The genetic differences between disomy and deletion include: (a) higher expression levels of maternally expressed genes in disomy, for genes in the PWS region; (b) haploinsufficiency of non-imprinted genes in this region, in deletion cases; and (c) loss of expression, in disomy, of any paternally expressed genes on chromosome 15 outside the Prader-Willi region (Bittel et al. 2003; Whittington et al. 2004). Thus, the UPD genotype exhibits a greater deviation towards increased relative expression of maternal genes. Biological similarities between Prader-Willi syndrome and psychotic-spectrum conditions include enlarged ventricles (Miller et al. 2007), altered serotoninergic and dopaminergic neurotransmission patterns (Akefeldt et al. 1998; Holsen \& Thompson 2004), impaired stereopsis (Chen et al. 2005; Fox et al. 1999), and a high pain threshold (Kuwako et al. 2005; Singh et al. 2006). Lee et al. (2005) postulated that "Prader-Willi syndrome is one of an emerging class of neurodevelopmental disorders that includes BBS [Bardet-Biedl syndrome], schizophrenia, and lissencephaly, which are in part caused by defects in centrosome function in cytoskeletal rearrangement during neurite extension" (p. 628). Neuroanatomically, PraderWilli syndrome is apparently mediated by impaired development of the hypothalamus (Goldstone 2004), the neurological nexus of the paternal brain.

Veltman et al. (2004; 2005) discuss the presence of autistic symptoms in Prader-Willi syndrome, which primarily involves obsessive behaviors and deficits in social interaction (e.g., social withdrawal), with language abilities largely intact. Such symptoms are about twice as common in uniparental disomy than deletion cases (Veltman et al. 2005), which is consistent with an alternative interpretation of these patterns as indicating expected aspects of a personality "premorbid" for schizophrenia, a condition which involves notable deficits in social and language development (Cannon et al. 1997; Sporn et al. 2004a; Vourdas et al. 2003). More generally, childhood diagnoses of autism in individuals with neurogenetic syndromes showing greatly increased rates of psychotic-spectrum disorders in adulthood, such as Klinefelter syndrome (Boks et al. 2007a; DeLisi et al. 2005; Jha et al. 2007) and velocardiofacial syndrome (Antshel et al. 2007; Gothelf 2007; Vorstman et al. 2006) may represent "false positives" (Feinsten \& Singh 2007), motivated by superficial childhood similarities between autism and "premorbid" psychotic-spectrum conditions (Eliez 2007) that are not underlain by genetic, neurological, or other biological criteria. Such considerations also apply to diagnoses of atypical autism in childhood, which Mouridsen et al. (2008) found to be followed in adulthood by diagnoses of "schizophrenia spectrum disorders" in 31 (35\%) of 89 cases. 
Symptoms of Angelman syndrome in childhood include prolonged suckling, frequent laughter, hyperactivity, and frequent waking (Badcock 2000; Cohen et al. 2005; Williams et al. 2006a). As in severe cases of autism, speech is often absent (Holm et al. 1993). Angelman syndrome also exhibits a disproportionately high rate of autistic traits that include deficits in reciprocal social behavior, poor eye contact, intolerance to change, and repetitive and stereotyped behaviors (Cohen et al. 2005; Peters et al. 2004; Schroer et al. 1998; Trillingsgaard \& Østergaard 2004). Peters et al. (2004) found that $42 \%$ of Angelman children in a long-term study met DSM-IV criteria for autism, and Sahoo et al. (2006) diagnosed 48\% as autistic, with a higher frequency (80\%) in cases of the larger, "Type 1" deletion at 15q11-q13. Angelman syndrome also involves mildly increased body weight in early childhood in three of the classes of genetic alteration that cause it (paternal UPD15, imprinting center alteration, and UBE3A mutation), as well as in some mouse models (Johnstone et al. 2006; Lossie et al. 2001). Further biological evidence for similarities between Angelman syndrome and autism includes high rates of seizures, an epileptiform EEG (electroencephalogram), and ataxia in both conditions (Williams et al. 2006a); genetic associations of UBE3A alleles with autism (Nurmi et al. 2001); and genetic models that posit a strong role for UBE3A dysregulation in autism (Jiang et al. 2004). An important contrast is macrocephaly in autism (Lainhart et al. 2006; Stanfield et al., in press), but acquired microcephaly in Angelman syndrome.

Taken together, the high rates of autistic-spectrum traits in Angelman syndrome, and psychotic-spectrum traits in Prader-Willi syndrome, suggest that diametric dysregulation of imprinted genes - towards increased paternal and maternal expression, respectively - mediates the expression of diametric behavioral and psychiatric phenotypes. By our hypothesis, individuals with Beckwith-Wiedemann syndrome should also show autistic features, and Silver-Russell syndrome should involve traits relatively characteristic of the psychotic spectrum.

\section{Diametric phenotypes of psychosis and autism}

The term autism was originally coined by Bleuler in the context of negative symptoms of schizophrenia, and Kanner (1965) struggled to establish autism as a disorder separate from childhood-onset schizophrenia until Kolvin's (1971) classic study showing bimodality in timing of onset for "childhood psychosis." The comorbidity of autism and schizophrenia is apparently low (Goussé et al. 2002). Leyfer et al. (2006) found no comorbid cases in a sample of 109 autistics, but they were ages 5-17, so few cases would be expected. (By contrast, depression, attentiondeficit/hyperactivity disorder [ADHD], and obsessive-compulsive disorder [OCD] were markedly elevated.) Volkmar and Cohen (1991) reported one case of schizophrenia in 163 adolescent and adult autistic individuals, which is at or below the general prevalence of about $1 \%$ in the overall population. Stahlberg et al. (2004) analyzed 129 adults (mean age 32) with autistic-spectrum disorders, and found no schizophrenia in 13 cases of autism, 1 case of schizophrenia, and 5 cases of "other psychotic disorder" in 49 Asperger syndrome cases, and 3 cases of schizophrenia and 1 case of "other psychotic disorder" in 67 cases of PDD-NOS (pervasive developmental disorder not otherwise specified). This latter association is of questionable salience to the hypothesis, given that Sporn et al. (2004a) have described a high incidence of PDD-NOS associated with later-onset schizophrenia.

The main complications of interpreting comorbidity studies are that apparent Asperger syndrome cases may involve "autistic" features expressed in some negative symptoms of schizophrenia and schizotypy (Konstantareas \& Hewitt 2001; Goldstein et al. 2002); the lack of communication skills in Kanner autism may make diagnosis of psychosis problematic; the presence of psychotic symptoms formally excludes an autism diagnosis by DSM criteria; and childhood "autism" may represent premorbidity for schizophrenia, as described earlier.

One of the strongest predictions of our hypothesis follows from the diametric nature of disruptions to systems affected by imprinted genes. Thus, the suite of phenotypic traits that characterize autistic- and psychotic-spectrum conditions should exhibit patterns of symmetrical and opposite phenotypes for traits related to growth and development, as well as aspects of social cognition and behavior. In this section, we describe evidence from studies of growth, development, neuroanatomy, cognition, behavior, and epidemiology for diametric phenotypes in autism and psychosis (Table 1). We focus on the most recent studies and comprehensive reviews, and we encourage neuroscientists, psychiatrists, and psychologists to consider the evidence as a convergent whole, constructively engage the core arguments and predictions, and suggest alternative possible explanations for the patterns that we describe.

\subsection{Growth and neuroanatomy}

6.1.1. Brain size, birth weight, growth, and placentation. Whole brain size is reduced in schizophrenia from birth onwards (Cannon et al. 2002; Gur et al. 2007; McIntosh et al. 2006; Tamminga \& Holcomb 2005), due to reductions in grey matter (neuronal tissue) (e.g., Narr et al. 2005; Woods et al. 2005), reduced and altered white matter (mainly brain fatty acids) (e.g., Kieseppä et al. 2003; McDonald et al. 2004; 2005), and lower cortical thickness (Goghari et al. 2007; Kuperberg et al. 2003). Moises et al. (2002) also noted that a considerable range of growth deficiencies, including low birth weight, late maturation, and small brain size, are found in schizophrenia. Reduced brain size may be due in part to slow brain maturation in individuals who develop psychosis (Crow 1995; Crow et al. 1996; James et al. 1999; Saugstad 1998; 1999).

In autism, cortical thickness is increased (Hardan et al. 2006), and increased head and brain size is one of the most consistent anatomical findings across studies (DiCiccoBloom et al. 2006; Dissanayake et al. 2006; Lainhart et al. 2006; Stanfield et al., in press). In autism, brain size undergoes a striking growth spurt between birth and age four (Cody et al. 2002; Courchesne \& Pierce 2005a; 2005b; Courchesne et al. 2004; Herbert 2005; Lainhart et al. 2006; Penn 2006; Redcay \& Courchesne 2005), an acceleration driven differentially by increases in (metabolically expensive) white matter volume (Herbert et al. 2004; Lainhart 2006; see also McAlonan et al. 2002). Remarkably, a recent study of Asperger syndrome 
Crespi \& Badcock: Psychosis and autism as diametrical disorders of the social brain

Table 1. Diametrically opposed phenotypes of autistic- and psychotic-spectrum conditions

[Note: Recent, salient references are indicated by number after each entry and collated at the bottom of the table. Full references are in the Consolidated References list, and discussion is provided in the target article main text.]

\begin{tabular}{|c|c|}
\hline Trait & Autistic Spectrum \\
\hline Placentation & Placental inclusions, associated with overgrowth \\
\hline Growth, anatomy & $\begin{array}{l}\text { High or same birth weight, length (mixed) }(4-6) \\
\text { Faster body growth in childhood }(5,10,11)\end{array}$ \\
\hline & $\begin{array}{l}\text { Lower } 2: 4 \text { digit ratio }(14,15) \\
\text { High levels of BDNF, IGF2 and other growth } \\
\text { factors }(18,19)\end{array}$ \\
\hline $\begin{array}{l}\text { Neuroanatomy and } \\
\text { neurological function }\end{array}$ & $\begin{array}{l}\text { Larger brain size, more gray and white matter, } \\
\quad \text { thicker cortex }(23-25) \\
\text { Lack of gray matter loss }(31) \\
\text { Larger amygdala (early development), more } \\
\quad \text { reactive }(33,34) \\
\text { Smaller corpus callosum }(34,36) \\
\text { Anomalous lateralization }(39,40) \\
\text { Relative right-hemisphere dysfunction }(43) \\
\text { Mirror neuron system underdeveloped }(46,47)\end{array}$ \\
\hline Cognition & $\begin{array}{l}\text { Deficits related to gaze }(49) \\
\text { Deficits in interpreting intention }(52) \\
\text { Deficits in shared attention }(55) \\
\text { Deficits in personal agency }(56,57) \\
\text { Theory of mind underdeveloped }(58,59)\end{array}$ \\
\hline
\end{tabular}

Hypoactivation in BA8,44,46 in functional imaging studies (64-67)

Reduced deactivation of resting network (71)

Literalness and inability to deceive (74)

Reduced imagination and symbolic play (76-78)

Abnormal filtering of sensory input, repetitive behavior, and focus on one or few aspects of environment $(81,82)$

No language in some Kanner autism cases; inner speech absent or reduced (86)

Enhanced visual-spatial skills relative to verbal skills $(88,89)$

Increased local processing (relative to global) (92)

Hyperlexia $(95,96)$

\section{Psychotic Spectrum}

Intrauterine growth restriction, placental undergrowth and fetal hypoxia $(2,3)$

Low birth weight, length (mixed) (7-9)

Slow growth, small size in childhood (mixed) $(2,12,13)$

Higher $2: 4$ digit ratio $(16,17)$

Low levels of BDNF and other growth factors $(20-22)$

Smaller brain size, less gray and white matter, thinner cortex $(26-30)$

Accelerated gray matter loss (32)

Smaller amygdala, less reactive (mixed) $(35,36)$

Larger corpus callosum (mixed) $(37,38)$

Less lateralized $(41,42)$

Relative left-hemisphere dysfunction $(44,45)$

Mirror neuron system dysregulated (48)

High gaze sensitivity, paranoia $(50,51)$

Delusions of persecution, erotomania $(53,54)$

Delusions of conspiracy (53)

Delusions of reference, megalomania (53)

Theory of mind overdeveloped in schizophrenia (60); enhanced theory of mind in healthy schizotypy (61-63)

Hyperactivation in BA8,44,46 (68-70)

Increased deactivation of resting network; more regions in network $(72,73)$

Delusional mentality, self-deception, magical ideation $(53,75)$

Complex social delusions; enhanced imagination $(79,80)$

Reduced filtering of stimulus relevance; "loose" associations between words and between aspects of the environment, enhanced creativity (83-85)

Auditory hallucination and thought insertion (87)

Deficits in some visual-spatial skills relative to verbal skills $(90,91)$

Increased global processing $(93,94)$

Dyslexia $(97,98)$

\footnotetext{
Key references: (1) Anderson et al. 2007; (2) Wahlbeck et al. 2001a; (3) Rees \& Inder (2005); (4) Sugie et al. 2005; (5) Mraz et al. 2007; (6) Dissanayake et al. 2006; (7) Nilsson et al. 2005; (8) Niemi et al. 2005; (9) Cannon et al. 2002; (10) Sacco et al. 2007; (11) Fukumoto et al., in press; (12) Gunnell et al. 2003; (13) Haukka et al., in press; (14) Manning et al. 2001; (15) Milne et al. 2006; (16) Arató et al. 2004; (17) Walder et al. 2006a; (18) Mills et al. 2007; (19) Connolly et al. 2006; (20) Moises et al. 2002; (21) Weickert et al. 2005; (22) Palomino et al. 2006; (23) Herbert et al. 2004; (24) Lainhart et al. 2006; (25) Hardan et al. 2006; (26) Kieseppä et al. 2003; (27) McDonald et al. 2005; (28) Tamminga \& Holcomb 2005; (29) McIntosh et al. 2006; (30) Goghari et al. 2007; (31) McAlonan et al. 2002; (32) Rapoport et al. 2005; (33) Schumann et al. 2004; (34) Stanfield et al., in press; (35) Aleman \& Kahn 2005; (36) Alexander et al. 2007; (37) Brambilla et al. 2005; (38) Tuncer et al. 2005; (39) Herbert et al. 2002; (40) Herbert et al. 2005; (41) Leask \& Crow 2005; (42) Weiss et al. 2006; (43) Gunter et al. 2002; (44) Mohr et al. 2001; (45) Hulshoff Pol et al. 2006; (46) Williams et al. 2001; (47) Hadjikhani et al. 2007; (48) Arbib \& Mundhenk 2005; (49) Ristic et al. 2005; (50) McKay et al. 2005; (51) Langdon et al. 2006b; (52) Gallese 2006; (53) Kimhy et al. 2005; (54) Kennedy et al. 2002; (55) Tomasello et al. 2005; (56) Toichi et al. 2002; (57) Grandin 2004; (58) Baron-Cohen \& Belmonte 2005; (59) Frith 2003; (60) Harrington et al. 2005a; 2005b; (61) Rim 1994; (62) Pilowsky et al. 2000; (63) Dinn et al. 2002; (64) Happé et al. 1996; (65) Luna et al. 2002; (66) Dapretto et al. 2006; (67) Silk et al. 2006; (68) Quintana et al. 2001; (69) Whalley et al. 2004; (70) Seidman et al. 2006; (71) Kennedy et al. 2006; (72) Garrity et al. 2007; (73) Harrison et al. 2007; (74) U. Frith 2004; (75) Camisa et al. 2005; (76) Losh \& Capps 2003; (77) Blanc et al. 2005; (78) Honey et al. 2006; (79) Claridge et al. 1990; (80) Nettle 2001; (81) Happé 1994; (82) Landry \& Bryson 2004; (83) Brugger \& Graves 1997a; (84) Brugger \& Graves 1997b; (85) Mathes et al. 2005; (86) Whitehouse et al. 2006; (87) Jones \& Fernyhough 2007; (88) Baron-Cohen et al. 2001; (89) Baron-Cohen et al. 2005; (90) Toulopoulou et al. 2005; (91) Kravariti et al. 2006; (92) Happé \& Frith 2006; (93) Bellgrove et al. 2003; (94) Sumich et al. 2005; (95) Just et al. 2004; (96) Turkeltaub et al. 2004; (97) Bersani et al. 2006; (98) Edgar et al. 2006.
} 
showed that grey matter volume did not decrease with age (from 15 to 50), as it does substantially in normal individuals (McAlonan et al. 2002; see also Ge et al. 2002; Woods et al. 2005); these data suggest that autism and schizophrenia exhibit divergent patterns of grey matter loss, with little to no loss in autism, moderate loss in normal development, and high rates of loss in schizophrenia.

These differences in brain size and development between autistic and schizophrenic individuals are broadly paralleled by differences in birth weight and growth. Thus, autism can involve higher birth weight compared to controls (Mraz et al. 2007; Sacco et al. 2007; Sugie et al. 2005) and faster body growth (Dissanayake et al. 2006; Fukumoto et al. 2008; Mraz et al. 2007), although some studies report a lack of birth weight difference (Cederlund \& Gillberg 2004; Juul-Dam et al. 2001; Larsson et al. 2005) or lower birth weight in autism (Kolevzon et al. 2007). By contrast, schizophrenia and major depression entail lower weight at birth with considerable consistency across studies (Cannon et al. 2002; Costello et al. 2007; Gale \& Martyn 2004; Gunnell \& Holly 2004; Niemi et al. 2005; Nilsson et al. 2005; Wahlbeck et al. 2001a). Imprinted genes are known to exert strong effects on birth weight and childhood weight in humans (Gorlova et al. 2003; Lindsay et al. 2002), and Svensson et al. (2006) have demonstrated familial aggregation of low birth weight, with effects from maternal, paternal, and fetal genes. The clearest evidence for enhanced growth in autism comes from Mills et al. (2007), who reported significantly increased head size, body weight, body mass index, and levels of growth hormones (including the key, paternally expressed growth factor IGF2) in autistic children compared to normal controls. Diametric patterns of growth can also help to explain the higher incidence of schizophrenia than autism, given that there should be many more genetic, epigenetic, and environmental ways to disrupt and reduce growth than to increase it. We also note that genomic conflicts and strong selection may contribute to the high heritabilities of autism and schizophrenia via such processes as antagonistic pleiotropy, evolutionary disequilibrium, and increased scope for mutation-selection balance via disruption of developmental tugs-of-war (Crespi 2006; Crespi et al. 2007).

Fetal development is critically dependent upon placentation, which in humans is highly invasive and has evolved in the context of constrained maternal-fetal conflict (Haig 1993). Anderson et al. (2007) recently described a highly significant, three-fold increase in placental inclusions in autism. Such inclusions are caused by increased proliferative growth of cytotrophoblast, the stem-cell-like component of the placenta. Placental inclusions are also found disproportionately in Beckwith-Wiedemann syndrome and hydatiform moles (molar pregnancies involving placental overgrowth), both of which represent disorders of genomic imprinting that involve excessive effects from paternal gene expression (Devriendt 2005; Fisher et al. 2002; Ohama et al. 1986; Saxena et al. 2003). Placental development in general is strongly regulated by imprinted genes, with paternally expressed genes favoring increased placental growth (Dunger et al. 2006; Fowden et al. 2006; McMinn et al. 2006; Reik et al. 2003).

Risk of schizophrenia decreases with increased placental weight (Wahlbeck et al. 2001a). Intrauterine growth restriction, which is caused predominantly by placental underdevelopment and engenders increased fetal hypoxia (Gagnon 2003), is also a strong risk factor for schizophrenia (Abel \& Allin 2006; Cannon et al. 2000; Rees \& Inder 2005). Effects of fetal hypoxia on brain development are also indicated by experiments in rats that link hypoxia with altered lateralization of the dopaminergic system (Brake et al. 2000). Finally, in humans, monozygotic twin concordance for schizophrenia is much higher when the twins share a placenta $(60 \%)$, than when they do not (11\%) (Davis et al. 1995). Abel (2004) describes additional evidence that fetal growth restriction, mediated by imprinting effects, contributes to the development of schizophrenia.

Placentation is crucial to brain development, and it represents a key arena for imprinted-gene conflict, because fetal brain growth, especially deposition of fatty acids, is one of the most metabolically costly processes during pregnancy, as well as exerting severe energetic costs in early postnatal development (Foley \& Lee 1991; Herrera 2002; Kuzawa 1998). Mothers bear virtually all of these costs, and indeed, during the late stages of pregnancy mothers metabolize their own brain fat for transfer to the fetus. We hypothesize that the contrasting patterns of brain size, growth, composition, and birth weight in psychosis and autism are mediated by effects of maternal versus paternal genes, with paternal genes driving the acquisition of increased brain fatty acids in particular. Directly parallel arguments have been made concerning intragenomic conflict over body fat in human babies (Haig 1999b): Human neonates exhibit by far the highest average body fat content of any mammal, which may represent an adaptation to sequester resources to fuel sustained brain growth in early childhood (Badcock 2000, pp. 208-212; Cunnane \& Crawford 2003; Kuzawa 1998). A role for imprinted genes in human fat metabolism is suggested by Silver-Russell syndrome, which is caused by a bias towards relative maternal gene expression and involves a striking lack of subcutaneous fat (Monk \& Moore 2004).

Patterns and predictions regarding early brain growth in autism and schizophrenia are critically important to the theory proposed here, because altered early brain growth rates are expected to strongly influence patterns of brain connectivity and cerebral lateralization, as described in detail in the next sections.

6.1.2. Hippocampus and amygdala size. The hippocampus is centrally involved in learning and the consolidation of memory, with the right side more involved in spatial cognition, and the left side dedicated more to aspects of memory (Burgess et al. 2002; Piefke \& Fink 2005). By contrast, the amygdala provides social and emotional valence to sensory perceptions, such as the recognition of fearful stimuli, providing input on emotional content to brain structures such as the hippocampus and neocortex (Adolphs et al. 2002; Sander et al. 2003; Skuse et al. 2003). Integrated activity of the amygdala, hippocampus, and social-brain components of the neocortex has been hypothesized as a core aspect of the social brain system, that processes the rapid, complex information flow involved in human interaction, especially interactions involving emotion, language, and facial expression. 
Autism and schizophrenia both involve alterations in structure and function of the interacting amygdala, hippocampus, and prefrontal cortex (Baron-Cohen et al. 2005; Berretta et al. 2001; Burns 2004; 2006a; Gisabella et al. 2005; J. D. Johnson 2005). The available evidence indicates that relative to brain size, the hippocampus and amygdala are larger in autism than in controls (at least during early development) (Schumann et al. 2004; Stanfield et al., in press), and (in most studies, and in adults) smaller in schizophrenia and schizotypy (Aleman \& Kahn 2005; Geuze et al. 2005; Gur et al. 2004; 2007; Kuroki et al. 2006; Lawrie et al. 2003; Narr et al. 2002; 2004; Suzuki et al. 2005; Tamminga \& Holcomb 2005; van Elst \& Trimble 2003). In schizophrenia, smaller size and altered shape of the hippocampus may be functionally related to positive symptoms such as paranoia and delusions, in that the hippocampus mediates the creation, maintenance, and updating of contextual social and spatial "worldviews" and beliefs, via interactions with the neocortex and amygdala (e.g., Gray 1998; J. D. Johnson 2005). In autism, increased hippocampus size may be related to enhanced visual-spatial, mathematical, and mechanistic aspects of cognition (Baron-Cohen et al. 2001; Minshew et al. 1997), as best seen in Asperger syndrome mechanistic skills, and the abilities of autistic savants at calculation and memory (Heaton \& Wallace 2004; Pring 2005; see also Young et al. 2004). Individuals with schizophrenia, and schizotypal individuals, exhibit cognitive profiles of impaired visual-spatial and arithmetic abilities, relative to verbal abilities, as described in more detail in the section on sex difference further on.

6.1.3. Cerebral lateralization. Schizophrenia involves reduced structural and functional brain asymmetry, as indicated by an increased incidence of mixed or inconsistent handedness, imaging studies of neuroanatomy with a focus on language-related regions such as the planum temporale, asymmetries in neurotransmitter activity, and higher impairments in verbal ability for individuals less lateralized for handedness (Chance et al. 2005; Collinson et al. 2003; Crow 1997; 1998; 2000; Crow et al. 1998; DeLisi et al. 2002; Honea et al. 2005; Leask \& Crow 2005; Mitchell \& Crow 2005; Schiffman et al. 2005; Shirakawa et al. 2001; Sommer et al. 2001; Weiss et al. 2006). This reduced brain lateralization and lower degree of torque in schizophrenia is apparently associated with slower brain development (Crow et al. 1996; 1998; Saugstad 1998; 1999), relatively increased dysfunction of components of the left hemisphere compared to the right (e.g., Honea et al. 2005; Kasai et al. 2003a; 2003b; Mucci et al. 2005) with diminished left-hemisphere specialization for language (Dollfus et al. 2005; Mitchell \& Crow 2005), and an increase in the extent of positive symptoms such as delusions (Verdoux et al. 2004). Similar patterns have been detected in healthy individuals, in whom the degree of schizotypal cognition is positively associated with mixed handedness and other evidence of reduced cerebral lateralization (Barnett \& Corballis 2002; Jaspers-Fayer \& Peters 2005; Preti et al. 2007; Shaw et al. 2001).

Impaired or reduced left-hemisphere language function in schizophrenia and schizotypy may result in greater reliance on right-hemisphere processing of some components of thought and language (Fisher et al. 2004;
Mohr et al. 2005; Taylor et al. 2002). A crucial result of such a shift may be more "coarse" semantic processing; generation of "loose," more-distant associations between events and thoughts (Pizzagalli et al. 2000); overestimation of meaningfulness of naturally occurring coincidences; increased paranormal ideation; and at the extreme, delusion, paranoia, and other positive symptoms of schizophrenia (Brugger 2001; Brugger \& Graves 1997a; 1997b; Leonhard \& Brugger 1998). This hypothesis is supported by a diverse range of additional evidence, from modelling of neural networks (Hoffman et al. 2004), to neurocognitive and psychological analyses of schizotypy (Claridge 1997), and the use of dopamine agonists to restore lefthemisphere language dominance (Mohr et al. 2005). The hypothesis also provides a relatively simple neuroanatomical and neurophysiological explanation for the links between creativity and psychosis as a cognitive style that involves more-distant and more-novel associations between components of thought (Barrantes-Vidal 2004; Brugger 2001; Gianotti et al. 2001). The general links of imagination and creativity with psychosis (Claridge et al. 1990; Nettle 2001; Sack et al. 2005) strongly contrast with the lower levels of pretend play and symbolic creativity, as well as increased repetitive and compulsive behavior, in autism (Atlas \& Lapidus 1987; Blanc et al. 2005; Boucher 2007; U. Frith 2004; Honey et al. 2006; Turner 1999).

Whereas schizophrenia is associated with reduced asymmetry, autism tends to involve increased size of some right-hemisphere cortical structures (i.e., reversed asymmetry compared to normal), and reversed lateralization of language (Bigler et al. 2007; De Fossé et al. 2004; Escalante-Mead et al. 2003; Flagg et al. 2005; Herbert et al. 2002; 2005; see also Rinehart et al. 2002b; Sutton et al. 2005). As the right hemisphere develops earlier than the left hemisphere in the fetal and neonatal stages (Chiron et al. 1995; see also Rinehart et al. 2002b), a pattern of rightward asymmetry in autism may be due simply to a faster, earlier pattern of brain development - a heterochronic shift opposite to a slower developmental profile in schizophrenia (Saugstad 1999). Accelerated early brain development in autism, and relatively slow brain development in schizophrenia, may thus both lead to anomalous lateralization of cognitive functions.

6.1.4. Corpus callosum size and brain connectivity. In autism, the corpus callosum is relatively small (for brain size) compared to control individuals (Cody et al. 2002; Egaas et al. 1995; Nydén et al. 2004; Piven et al. 1997; Sherr et al. 2005; Stanfield et al., in press; Waiter et al. 2005). A pattern of reduced interhemispheric brain connectivity in autistic individuals (Belmonte et al. 2004a; 2004b; Nydén et al. 2004) fits with one of the central hypotheses for the cognitive architecture of autism: that it involves increased local and decreased global information processing, reduced "central coherence," increased "bottom-up" relative to "top-down" processing, and decreased overall brain connectivity (Baron-Cohen \& Belmonte 2005; Belmonte et al. 2004a; 2004b; Happé \& Frith 2006; Just et al. 2004). Courchesne and Pierce (2005b) have referred to autistic neurocognition as the frontal cortex unconsciously "talking to itself," concomitant with loss of language and other elements of social cognition. 
Crespi \& Badcock: Psychosis and autism as diametrical disorders of the social brain

Findings regarding corpus callosum size in schizophrenia are mixed and inconsistent, apparently due to marked variation with age, sex, handedness, and clinical profile (Bachmann et al. 2003; Brambilla et al. 2005; Downhill et al. 2000; Highley et al. 2003; Luders et al. 2003; Tuncer et al. 2005). The issue of brain connectivity in schizophrenia has yet to reach a consensus among researchers, as it has for autism, perhaps due to the high clinical heterogeneity in schizophrenia. A considerable body of evidence suggests that schizophrenia involves altered intrahemispheric and interhemispheric connectivity, but such changes take particular forms, such as altered transfer of verbal information between hemispheres (Barnett \& Kirk 2005; Barnett et al. 2005; Endrass et al. 2002), that are difficult to interpret without a cognitive model for their significance. One possibility suggested by neural network models, and consistent with increased relative corpus callosum size, is that schizophrenia involves reduced local (relative to global) connections (Siekmeier \& Hoffman 2002), which would provide a contrast with the enhanced local connectivity and processing found in autism (Courchesne \& Pierce 2005a; 2005b; Happé \& Frith 2006). This hypothesis is supported by several findings, including: (1) the results of Whalley et al. (2005), who recorded increased connectivity of left parietal with left prefrontal regions in individuals at high risk of schizophrenia; (2) hyperconnectivity across brain regions for some (though not other) EEG frequencies in schizophrenia (Sritharan et al. 2005; Strelets et al. 2002; see also McCreery \& Claridge 1996); and (3) coactivation of inner speech and language regions normally activated in sequence (Hubl et al. 2004) and inferred wider-spreading activation of brain regions in schizophrenia (Chance et al. 2005) and schizotypy (Pizzagalli et al. 2001; see also Niebauer 2004). Sumich et al. (2005) provide MRI evidence that unreality symptoms and hallucinations in schizophrenia involve dysfunctions in Heschl's gyrus that impair bottom-up processing, "giving greater perceptual control to 'top-down' mechanisms" (p. 947); Seal et al. (2004) provide evidence for "heightened influence of top-down processing on perception" in auditory hallucinations of schizophrenics; and Carter et al. (1996), Granholm et al. (1999), and Bellgrove et al. (2003) have shown that schizophrenia involves greater impairments in local as opposed to global processing of stimuli, and exaggerated global-processing advantages for some tasks.

Finally, the neuroanatomical and cognitive correlates of dyslexia are notably similar to those found in schizophrenia and schizotypy (Bersani et al. 2006; Bradshaw \& Nettleton 1983, pp. 242-54; Edgar et al. 2006; Heim et al. 2004; Richardson 1994), and dyslexia has also been linked with "enhanced ability to process visual-spatial information globally (holistically) rather than locally (part by part)" (von Károlyi et al. 2003). By contrast, hyperlexia, the spontaneous and highly precocious mastery of reading in children (Grigorenko et al. 2003; Silberberg \& Silberberg 1967; 1971), is found almost exclusively in conjunction with autism (Burd \& Kerbeshian 1988; Just et al. 2004; Turkeltaub et al. 2004). The neural basis for hyperlexia was investigated in a single case study using fMRI: Hyperlexic reading was associated with hyperactivation of the left superior temporal cortex, a region that is hypoactivated in dyslexia (Turkeltaub et al. 2004).

\subsection{Neurodevelopment}

Many of the diverse traits in Table 1 are developmentally and functionally related, with a central role for coordinated allometric development of brain size, relative sizes of brain regions, brain grey and white matter composition, intra- versus inter-hemispheric connectivity, corpus callosum size, and cerebral asymmetry (Burns 2006a). Thus, autism commonly involves an acceleration of brain development, increased childhood brain size (especially in the frontal lobe), and relatively increased white matter (Carper et al. 2002; Courchesne 2004; Courchesne \& Pierce 2005a; 2005b; McCaffery \& Deutsch 2005; Schumann et al. 2004). In humans and other primates, increased overall brain size normally involves an increased relative proportion of white matter (Schoenemann et al. 2005), relatively increased intra-hemispheric (compared to inter-hemispheric) connectivity (see Burns 2006a), and a corpus callosum small relative to brain size (Rilling \& Insel 1999; Vidal et al. 2006). Brain-growth acceleration in autism may also engender faster growth of the developmentally leading right-hemisphere prior to age three (see Chi et al. 1977; Chiron et al. 1995; Pilcher et al. 2001; Seldon 2005), resulting in a higher frequency of reversed (rightward) asymmetry especially in language areas such as the planum temporale, and contributing to the general pattern in autism of apparent relative deficits in right-hemispheric and relatively global tasks (Gunter et al. 2002; Previc 2007). By contrast, as described earlier, schizophrenia apparently involves slower early brain development, smaller brain size, and relative reductions in white matter, which may lead to differentially impaired development of the slower-developing left hemisphere (e.g., Crow 1997; Flor-Henry 1969; Galaburda 1984; Harrison 1999; Hulshoff Pol et al. 2006; Mohr et al. 2001). This contrast between relative left hemisphere dysfunction in schizophrenia (involving relative deficits in sequential, serial, and more-local encoding and processing tasks) versus relative right-hemisphere impairment in autism (involving relative strengths in some local and linear processing tasks but relative deficits in gestalt, more-global, holistic tasks such as social interaction; see Bradshaw \& Nettleton 1983, pp. 162-72) is a considerable oversimplication, but it potentially helps to link dysregulated early brain growth rates with important aspects of lateralized cognition (Han et al. 2002). We stress that both autism and schizophrenia involve absolute deficits in functions ascribed to both hemispheres - for example, schizophrenics show impaired pragmatics of language (Langdon et al. 2002) - and that the "normal" functions of the two hemispheres may not always be unambiguously attributed to their usual cerebral locations in these two conditions.

The hypothesis that autism involves general effects of accelerated early brain development, and psychosis involves slower development, is supported by three additional lines of evidence.

First, autism involves overactivation of some growthsignalling pathways and some key brain growth factors such as BDNF (Carper et al. 2002; Connolly et al. 2006; McCaffery \& Deutsch 2005; Miyazaki et al. 2004; Nelson 2001; Nishimura et al. 2007a; Tsai 2005). In contrast to autism, schizophrenia involves deficiencies in growth factors (Gunnell \& Holly 2004; Hashimoto et al. 
2005; Klejbor et al. 2006; Moises et al. 2002; Niculescu 2005; Weickert et al. 2003; 2005), which can in some cases be traced directly to the disorder (e.g., Pieper et al. 2005).

Second, undergrowth versus overgrowth effects on human brain development are strongly mediated by the phosphatidylinositol 3-kinase (PI3K) signalling pathway (Brunet et al. 2001), which is strikingly downregulated in schizophrenia and bipolar disorder via alterations in growth-enhancing genes (Emamian et al. 2004; Kalkman 2006; Stopkova et al. 2004) but exhibits hyperactivation in some autistic conditions due to reduced expression or activity of genes that negatively regulate $\mathrm{PI} 3 \mathrm{~K}$ pathway activation, including PTEN, NF1, TSC1, TSC2, and PRKCB1 (Belmonte \& Bourgeron 2006; Butler et al. 2005; Crespi, under revision; Kwon et al. 2006; McCall et al. 2006; Philippi et al. 2005; Serajee et al. 2003). In turn, PI3K signalling is mediated in part by the growthregulating imprinted genes GRB10, IGF2, H19, and GNAS (Charalambous et al. 2003; Chen et al. 2004; Deng et al. 2003; Fults 2005; Hartmann et al. 2005; Mahmoud \& Grover 2006; Riedel 2004; Smith et al. 2006; Wick et al. 2003).

Third, brain development may also be strongly affected by altered thresholds for apoptosis and synaptic pruning, as excessive pruning during adolescence has been suggested as a strong risk factor for schizophrenia (Burns 2004; de la Fuente-Sandoval et al. 2005; Walker \& Bollini 2002; Woo \& Crowell 2005). By contrast, several studies suggest that decreased apoptosis may be related to the accelerated early neurodevelopment found in autism (Courchesne \& Pierce 2005a; Engstrom et al. 2003; Fatemi \& Halt 2001). We suggest that the pathology of negative symptoms of schizophrenia in particular may be mediated by excessive loss of synapses and grey matter, caused in part by a bias towards maternally expressed imprinted genes, many of which act as tumor suppressors that enhance apoptosis, whereas paternally expressed genes enhance cell survival and growth (e.g., Kurita et al. 2006; Margetts et al. 2005).

The hypothesis that neurodevelopment is accelerated in autism but slowed in schizophrenia is congruent with neurodevelopmental models of these conditions (Courchesne \& Pierce 2005a; 2005b; Kalkman 2006; Rapoport et al. 2005; Redcay \& Courchesne 2005). The hypothesis also fits with expectations from the conflict theory of imprinting: That autism, driven by imbalance towards increased effects of paternal gene expression, involves faster growth and increased demands on the mother, whereas psychosis engenders the reverse. We have illustrated this hypothesis in Figure 3, which depicts its continuity with classic neurodevelopmental models. Further analyses of this neurodevelopmental hypothesis require demonstrating effects of imprinted genes on early brain growth, altered lateralization (e.g., Francks et al. 2007; Sun et al. 2005), and position along a cognitive spectrum from autism to psychosis.

\subsection{Cognition}

Diverse hypotheses have been proposed to explain how neurological functions are altered in psychotic and autistic-spectrum conditions (e.g., Baron-Cohen et al. 2005; Brüne 2004; Burns 2004; Gray 1998; Happé \&
Frith 2006; J. D. Johnson 2005). A point of near-consensus in these ideas is that cognitive dysfunctions are driven by altered interactions between and within components of the human social brain, which comprises the amygdala, superior temporal sulcus, orbitofrontal cortex, anterior congulate cortex, temporoparietal junction, temporal poles, medial prefrontal cortex, and the mirror-neuron systems (Brüne 2004; Burns 2004, 2006a; Happé \& Frith 2006). Our hypothesis predicts that integrated social brain systems are disrupted in diametrically opposite ways in autistic- and psychotic-spectrum conditions. This hypothesis can be evaluated via analysis of how aspects of gaze, intentionality, agency, theory of mind, and the mirror-neuron systems are underdeveloped or dysregulated in autism and in schizophrenia, the condition with the most information available.

6.3.1. Gaze and intention. Humans are unique among primates in exhibiting a white sclera (the "whites of the eyes") (Emery 2000), which allows direct observation of the form and directionality of gaze (e.g., Whalen et al. 2004). Gaze is a fundamental component of social interactions, with most humans exquisitely cognizant of the gaze of others and perceptive of direction and form of gaze, and expressions around the eyes, as conveying a considerable amount of social information concerning mental state and intention (Emery 2000). Specific neural regions, in the neocortex and amygdala, are dedicated to perception and interpretation of gaze and facial expression in humans and other primates (Emery 2000; M. H. Johnson 2005). The amygdala in particular is highly activated when humans gaze upon one another, as emotional valence of the interaction is inferred from features of the eyes and surrounding face, with especially notable sensitivity to fear (Adolphs et al. 2005; Castelli 2005; Schwartz et al. 2003a; 2003b; Whalen et al. 2004).

Autistic individuals exhibit notable avoidance of gaze, and reduced or absent following of gaze (Asperger 1991; Emery 2000; Frith 2003, p. 105; Ristic et al. 2005). Such behavior has motivated the development of theories for autism based on impairment of the amygdala (as in cases of amygdala damage), with consequent reduction in ability to detect and assimilate social and emotional information from the eyes and faces of other humans (Adolphs et al. 2002; Baron-Cohen et al. 2000; Brambilla et al. 2004; Shaw et al. 2004). First-person accounts by autistic individuals (Lawson 1998, pp. 11-12; O’Neill 1999, p. 26) suggest that amygdala "impairment" may involve hyperactivation, such that direct gaze is sufficiently uncomfortable, overwhelming, and fear-inspiring to warrant avoidance. This inference is also supported by the larger size of the amygdala in autism (at least during early childhood) (Schumann et al. 2004), lower levels of the fearreducing neuropeptide oxytocin in autism (Green et al. 2001; Kirsch et al. 2005), the ability of autistic children to recognize basic emotions (though not social, self-conscious emotions) from eyes and facial features (Castelli 2005; see also Adolphs et al. 2005), reduced inhibition of the amygdala in autism (Rubenstein \& Merzenich 2003), a functional-imaging study that shows heightened amygdala activation in response to gaze fixation in autism (Dalton et al. 2005a), and relatively strong neurological links between the amygdala and the right hemisphere in normal subjects compared to autistics (Noesselt et al. 
Crespi \& Badcock: Psychosis and autism as diametrical disorders of the social brain

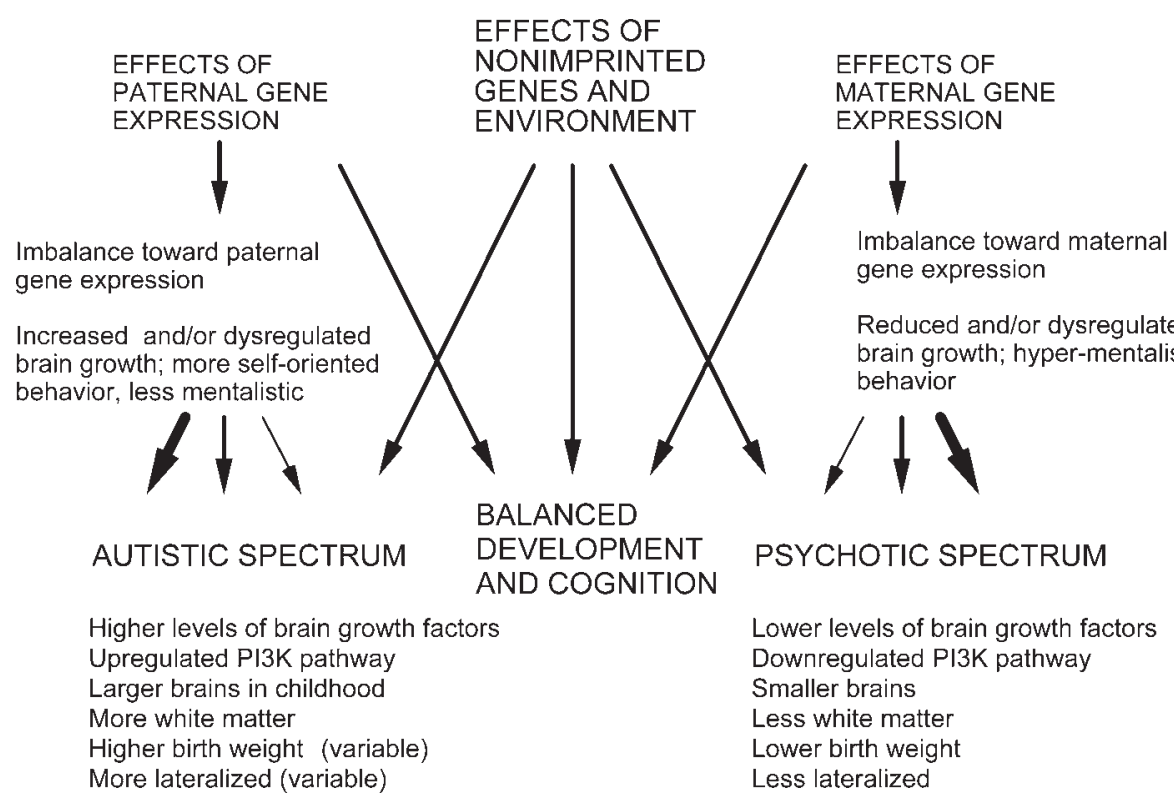

Figure 3. Our neurodevelopmental model of autism and psychosis is compatible with previous theory and data on dysregulated neurodevelopment in these two sets of conditions, which posits diverse effects from many genes as well as environmental effects (e.g., valproic acid; see Rinaldi et al. 2008), but it contrasts these conditions as diametric opposites modulated by differential brain growth and development, with notable effects from genetic and epigenetic alterations of imprinted genes. The degree to which imprinted and non-imprinted genes contribute to effects on brain growth and development remains unclear, but only imprinted genes are expected to exert effects on cognitive architecture that reflect dysregulated adaptations related to the conflict hypothesis of imprinting.

2005). Long-term avoidance of gaze and faces presumably reduces opportunities for autistic children to develop skills related to this core component of social behavior, via cognitive appraisal of eye and facial features during interactions (Gläscher \& Adolphs 2003; M. H. Johnson 2005; Skuse et al. 2005; see also Adolphs et al. 2005). Such deficits, as well as other components of the social brain, may be involved in the underdeveloped theory of mind found in Kanner autism and to a lesser extent in Asperger syndrome (Baron-Cohen 2002; 2003; Bowler 1992; Downs \& Smith 2004).

Langdon et al. (2006b) demonstrated that schizophrenia involves abnormal over-responsiveness in attentional orienting to gaze (see also Emery 2000; Franck et al. 1998), which is the opposite pattern to that observed in autism. Although impairments in tests of recognizing expression from eyes and faces have been described in schizophrenia (e.g., Kington et al. 2000) as for autism, such deficits appear to be a function of inaccurate inferences concerning gaze of others as directed towards them (Hooker \& Park 2005), and mistaken inference of mental states from gaze (Langdon et al. 2006a; 2006b), rather than the underdevelopment or absence of mental-state attribution ascribed to autistic cognition (Baron-Cohen 2002; Craig et al. 2004; Frith 2003). Paranoia, a prominent feature in the positive symptoms of psychosis, also commonly involves obsession with the gaze of others as directed towards one's self, often concomitant to delusions of persecution and conspiracy (e.g., Badcock 2004; Franck et al. 1998; Green \& Phillips 2004; LaRusso 1978; McKay et al. 2005).

We interpret these features of psychosis as forms of hyper-mentalizing, such that theory of mind is dysregulated via impaired, inflexible, or extreme inferences regarding social cues and over-attribution of mental states and intentions (Abu-Akel 1999; Abu-Akel \& Bailey 2000; Badcock 2004; Blackwood et al. 2001; C. D. Frith 2004; Harrington et al. 2005a; 2005b; Langdon \& Coltheart 1999; Langdon et al. 2006a; Phillips et al. 2000; Steiner 2004). Indeed, the evolutionary psychiatrist Randolph Nesse noted that "those who have worked with schizophrenics know the eerie feeling of being with someone whose intuitions are acutely tuned to the subtlest unintentional cues, even while the person is incapable of accurate empathic understanding" (Nesse 2004, p. 62), and Claridge et al. (1990, p. 221) noted that "anyone who has interacted with psychotics will know of their uncanny capacity to respond to subtle social cues, believed to have been concealed from them." These patterns contrast with the extreme literal-mindedness found in autistic individuals, in comparison to the common overinterpretation of linguistic and visual input in schizophrenia and to some degree in schizotypy (Langdon \& Coltheart 2004; Langdon et al. 2002; Russell et al. 2006), although schizophrenia can also involve deficits that engender impaired processing of figurative language (Thoma \& Daum 2006). Both autistic literal-mindedness and mechanistic cognition, and psychotic-spectrum overinterpretation can lead, though by different mechanisms, to deficits in theory of mind tasks and understanding of pragmatic language such as metaphor and humor (C. D. Frith 2004; Frith \& Allen 1988; Mitchell \& Crow 2005). Taken together, this evidence suggests that reaction to gaze, as well as tendency to attribute mental states and intentions to others, exhibit contrasting patterns of hyperdevelopment and underdevelopment in psychosis and autism (Table 1). 
Crespi \& Badcock: Psychosis and autism as diametrical disorders of the social brain

The neurophysiological and psychological mechanisms underlying hyperdevelopment of gaze, inference of intention, and theory of mind in schizophrenia require further study. As in autism, the amygdala plays an important role in the interactions that mediate theory of mind skills in schizophrenia (Benes \& Berretta 2001; Brüne 2004; Brüne \& Brüne-Cohrs 2006). Many (though by no means all) functional-imaging studies demonstrate that schizophrenia engenders underactivation of the amygdala compared to controls in tests of recognizing facial expression and emotional valence (Brunet-Gouet \& Decety 2006; Das et al. 2007; Gur et al. 2002; Holt et al. 2006; Kucharska-Pietura et al. 2003; Paradiso et al. 2003; Phillips et al. 1999; Schneider et al. 1998). These findings are consistent with the emotional dysregulation commonly found in schizophrenia (Aleman \& Kahn 2005), although the connections between emotion and cognition in this and other psychotic-spectrum conditions remain unclear. Underactivation of the amygdala may also be a manifestation of reduced cognitive effects from the paternal brain (Keverne 2001a; 2001b) in negative-symptom schizophrenia.

Finally, gaze and intentionality are intimately associated with the expression of emotion, which is generally recognized as reduced in schizophrenia and major depression (e.g., Gaebel \& Wölwer 2004; Troisi et al. 2007), although lack of facial expression need not indicate a lack of emotional experience (Trémeau et al. 2005); indeed, a relatively high level of positive schizotypy appears to be associated with more intense emotionality (Kerns 2005). Parental reports provide evidence that autistic children are highly expressive, especially for negative emotion, although they "communicated feeling states idiosyncratically, in a manner only their mother understood" (Capps et al. 1993) (p. 475). Losh and Capps (2006) also report that autistic children are no less emotionally expressive than controls for basic emotions, though not self-conscious ones. In autism, levels of basic emotionality may thus be as high or higher than normal (in keeping with relatively enhanced paternal-brain functions), but emotional reactions lack social context and sensitivity due to reductions in the ability of the neocortical, maternal brain to integrate social-cognitive understanding and control with emotion and behavior.

6.3.2. Agency and self. Monitoring of gaze and inference of intention involve application of theory of mind skills to other individuals (Emery 2000; Tomasello et al. 2005). The other domain for theory of mind is reflexive - that is, selfconsciousness, mentalized awareness of one's self as an agent acting upon the external world, and exhibiting spontaneous self-directed thought. Several lines of evidence suggest that autism involves a diminished or altered sense of self: (1) selective impairments of episodic and autobiographical memory - the mental constructs that generate and maintain a sense of self (Gardiner 2002; Gardiner et al. 2003) - while rote, factual memory is spared or enhanced (O'Shea et al. 2005; Toichi \& Kamio 2002; Williams et al. 2006b); (2) a tendency to think in visual pictures (Grandin 1995; Hurlburt et al. 1994; Kana et al. 2006), suggesting a shift to the right hemisphere for brain regions generating spontaneous thought (Christoff et al. 2004); (3) a reduced sense of self-consciousness and personal agency, perhaps due to reduced central coherence at this level (Ben Shalom 2000; Fitzgerald 2005, p. 78; Frith \& Happé 2005; Grandin 1995; 2004; Johnson 2003; Lawson 1998, p. i; Lombardo et al. 2007; Toichi et al. 2002); (4) a less developed imagination, as noted earlier (Craig \& Baron-Cohen 1999; Happé 1994, p. 37; Losh \& Capps 2003); and (5) less developed experience of social and self-conscious emotion (Heerey et al. 2003; Losh \& Capps 2006). Autism also involves cognition highly focused on few particular non-social aspects of the environment, which is expressed in repetitive behavior, intense preoccupations about specific and narrow topics, and difficulties in shifting attention (Courchesne et al. 1994; Goldstein et al. 2001a; Happé 1994; Landry \& Bryson 2004; Murray et al. 2005). These features may interact via positive feedback to reduce the degree to which these individuals become socially enculturated (Murray et al. 2005), a central process in human development.

In contrast to autism, schizotypy and schizophrenia are characterized by high levels of distractability, reduced filtering of sensory input, and "loose" associations between external stimuli and between components of thought (e.g., Bellgrove et al. 2003; Brugger \& Graves 1997a; 1997b; Claridge \& Beech 1995; Grossberg 2000b; Mathes et al. 2005; Nakamura et al. 2003; Pizzagalli et al. 2000). The positive symptoms of schizophrenia often involve delusions, such as persecution and grandiosity, that "reflect individuals' preoccupations about their position in the social universe" (Bentall 2003b, p. 293) in creating complex and fanciful mental autobiographies (Salazar-Fraile et al. 2004), and an increased awareness of self and intentionality is found in schizophrenic delusions of control (Frith 2005b). Social and reflexive preoccupations can be described in terms of exaggerated selfconsciousness (Sass \& Parnas 2003), and indeed, auditory hallucination, thought insertion, and passivity phenomena (e.g., Behrendt 2004; Crow 2004a; 2004b) can be interpreted as functions of a breakdown in cognitive distinction between self and other, as in some spiritual experiences of schizotypal individuals (Brugger 2001). Breakdowns in self-monitoring, such as interpreting inner speech as external, or delusions of control of hand movements, have an apparent neurological basis in impaired ability to selfmonitor the coordination of intended (premotor) actions (mainly speech and gesture) with one's current and predicted state (Frith et al. 2000; Jones \& Fernyhough 2007). By contrast, autism appears to involve absent or reduced use of inner speech (Whitehouse et al. 2006).

Finally, auditory hallucinations commonly involve social dialogue, commentary, and commands, with a focus on complex, self-conscious emotions such as shame, contempt, derogation, and embarrassment (e.g., Legg \& Gilbert 2006); Bentall (2003a, p. 354) notes how such hallucinations often involve the voices of "significant family members," and Birchwood et al. (2004) describes them as operating "like external social relationships." These patterns, and the common feelings of guilt, deserved punishment, and worthlessness in major depression (Keller et al. 2007), suggest that social emotions and interactions are often overdeveloped in psychotic-spectrum disorders, in contrast to their reduction in autism, as described earlier.

6.3.3. Functional imaging of the social brain. The neurological basis for dysregulation of gaze, intention, agency, 
and theory of mind in autism and psychosis provides a mechanistic level of analysis that should dovetail with psychological descriptions of these processes. Thus, autistic- and psychotic-spectrum disorders may be expected to exhibit contrasting patterns of brain activation in functional-imaging studies that involve analyzing specific components of the social brain, as described earlier for the amygdala. We have found evidence for specific contrasts involving four brain regions or networks, although differences in the tasks utilized limit the strength of inferences in some cases.

First, Greicius et al. (2003) have described the brain's "default mode" or "resting state" network, which includes the medial prefrontal cortex, posterior cingulate cortex, ventral anterior cingulate cortex, and precuneus. This midline-structure network exhibits high metabolic rate at rest, with functions related to self-referential processing, processing of information related to theory of mind, inner speech, retrieving and manipulating memories, and developing future plans (Garrity et al. 2007; Greicius et al. 2003; Kennedy et al. 2006). This network is then "deactivated" when attention is redirected to a specific cognitive task. Kennedy et al. (2006) have described a "failure to deactivate" in autistics, which they attribute to a lower level of baseline resting-state activity, and default-mode cognition directed more towards obsessive interests and sensory-environment processing than towards self-reflective, theory-of-mind, and memory-retrieval activities. Moreover, a higher level of social impairment was associated with a lower degree of deactivation among subjects, which suggests a direct link between social cognition and activation of the resting network. Ohnishi et al. (2000) and Castelli et al. (2002) also found reduced activation in autism for regions involved in theory of mind, notably the medial prefrontal cortex.

In contrast to these results for autism, Garrity et al. (2007) and Harrison et al. (2007) have described greater task-induced deactivation in schizophrenics than in controls, for some regions. Garrity et al. (2007) have also reported that stronger positive symptoms are associated with greater deactivation (due to increased activity at rest), and that schizophrenics included a larger area of the parahippocampal gyrus in the default mode than did controls. Higher deactivation has also been reported in anxiety disorder than in controls (Zhao et al. 2007); and in major depression, a specific, affect-processing region of the default network, the subgenual cingulate, exhibits increased metabolic rate and higher functional connectivity with the medial prefrontal cortex than in controls (Greicius et al. 2007), which the authors ascribe to increased self-referential and emotional processing in this condition. This set of studies suggests that psychoticspectrum conditions may involve alterations in restingstate network activation specific to increased mentalistic functions, and that they also show a pattern of greater deactivation of the default network than in controls or in autism.

Second, one of the primary areas involved in mental attribution and processing of agency is Brodmann's area (BA) 8 in the dorsomedial frontal cortex (Finger et al. 2006; Fletcher et al. 1995; Goel et al. 1995; Marjoram et al. 2006). Increased activity in this region has been associated with increased genetic risk of schizophrenia by Whalley et al. (2004), and Frith (1996) describes evidence that this region is involved in auditory hallucinations in schizophrenia. By contrast, Happé et al. (1996) found normal activity in this area among controls in a theory-of-mind task, but a complete lack of activation in an Asperger syndrome group.

Third, Luna et al. (2002) and Silk et al. (2006) described reduced activation in autism for BA 46 in the dorsolateral prefrontal cortex in a spatial working memory task, which the former authors interpreted in terms of reduced "topdown" executive function. By contrast, significantly increased activation of right-hemisphere BA 46 was found by Seidman et al. (2006) in adolescents at high risk for schizophrenia, and these authors describe parallel findings of exaggerated activity in this region from three previous studies. A role for this region in social cognition is indicated by Knoch et al. (2006), who found activation in this area during implementation of fairness-related behaviors in social-reciprocity tasks.

A final set of core regions of the social brain is the mirror neuron systems. These systems, comprising regions of the superior temporal sulcus, inferior prefrontal cortex, inferior parietal cortex, and other regions, provide neural substrates for inference of intention, theory of mind and empathy, via activation of the same premotor circuitry during observation and execution of specific facial, manual, or other movements (Iacoboni \& Dapretto 2006). Such matching of premotor to observed motor activations allows for automatic inferences regarding the intention, disposition, and emotional state of another individual, which can generate cognitive and emotional resonance during social interactions. In autism, a key component of this system, BA 44 (the pars opercularis), was hypoactivated in tasks involving imitation or viewing emotional expressions (Dapretto et al. 2006); by contrast, Quintana et al. (2001) found overactivation of BA 44 in schizophrenia compared to controls for tasks involving affective facial expression, which they described in terms of "increased mirror-like representational mechanisms . . . with cues of obvious social value" (p. 923).

Hadjikhani et al. (2007) and Dapretto et al. (2006) describe evidence that face- and gaze-processing deficits in autism are due to impairments in the integrated activity of the mirror-neuron system use in facial processing. Patterns of hypoactivation in mirror-system regions in autism are paralleled by evidence for cortical thinning of these areas in autism, which is directly related to the severity of autistic symptoms (Hadjikhani et al. 2006; Zilbovicius et al. 2006). Reduced activation of mirror neuron systems in autistics appears specific to tasks dependent upon mentalistic cognition, rather than representing a more general impairment that also includes conscious, deliberate imitation or inference of intention from functional gestures (Hamilton et al. 2007).

Taken together, these findings indicate that underdevelopment of the integrated social brain in general, and social aspects of the mirror neuron system in particular, are associated with some of the social-behavioral deficits found in autism (Williams et al. 2001; cf. Dapretto et al. 2006; Hadjikhani et al. 2006; 2007). Schizophrenia also clearly engenders impairments in theory-of-mind skills and mentalizing (Brunet-Gouet \& Decety 2006; Harrington et al. 2005a; 2005b; Pinkham et al. 2003), which commonly involve "over-mentalizing," such as delusional ideation, inferring false intentions, and a general pattern 
Crespi \& Badcock: Psychosis and autism as diametrical disorders of the social brain

of confabulation of subjective experience in the face of misinterpreted objective reality (C. D. Frith 1992; 2004; Frith \& Frith 1999). Arbib and Mundhenk (2005) link such impairments to the mirror neuron system, in suggesting that functional dissociations between action or speech imagination, and enactment of movement or speech, lead to misattribution of agency and consequent confabulation and rationalizing, which manifests as auditory hallucination, delusions, and paranoia. Similar considerations may apply to the mirror-neuron system underlying face perception and emotional resonance, which is also dysregulated in schizophrenia in the context of emotion inappropriate to social context and flat affect (e.g., Aleman \& Kahn 2005; van Rijn et al. 2005). A recent review of functionalimaging studies of social brain dysfunction in schizophrenia also suggests that two mirror-neuron regions - the inferior frontal cortex and the inferior parietal lobe (see Arbib \& Mundhenk 2005) - are selectively responsible for some core cognitive manifestations of this disorder, as well as strongly implicating the medial prefrontal cortex, anterior cingulate cortex, and amygdala (Brunet-Gouet \& Decety 2006).

These findings indicate that in contrast to autism, where the mirror-neuron system does not develop to full, integrated functional maturity, in schizophrenia this system develops but is subject to diverse forms of selective malfunction. Thus, aspects of theory of mind, and mirrorneuron system skills, are selectively impaired in both autism and schizophrenia (Brüne \& Brüne-Cohrs 2006; C. D. Frith 1992; 2004; Lee et al. 2004; Mazza et al. 2001; Pickup \& Frith 2001; Russell et al. 2006; ShamayTsoory et al. 2007; see also McCabe et al. 2004), but, we believe, for different reasons.

\subsection{Behavior and adaptive significance}

Conflicts involving imprinted genes, and mother-offspring conflict more generally, are most obvious during prenatal and early childhood development where fitnesslimiting resources from placenta and breast can be quantified. But how does one address and quantify costs imposed via cognition and behavior, after early infancy? One prediction of the imprinted brain hypothesis for autism and psychosis is that an increased tendency towards autistic traits entails higher costs imposed on mothers, and that healthy schizotypal behavior in offspring might benefit the mother and other salient maternal relatives, presuming that pathological effects of altered development are not too extreme.

The autistic spectrum, which literally refers to "selfishness" in cognition, involves a large suite of traits that can be interpreted as imposing additional costs on mothers (Badcock \& Crespi 2006). Indeed, each of the three main classes of behavior that define autism, (1) impaired social interaction, (2) impaired language development, and (3) repetitive behavior and insistence on sameness, are likely to engender increased demands. Some prevalent traits, such as tantrums, attempts to control others, lack of cooperative behavior, and the notable lack of empathy that characterizes Asperger syndrome (Arbelle et al. 1994; Asperger 1991; Baron-Cohen 2002; Lawson et al. 2004; Soderstrom et al. 2002), appear especially demanding of maternal time and other resources. Similar considerations are expected to apply to non-clinical individuals on the autistic spectrum, showing to some degree any of the traits in Figure 1.

The development of autistic behavior in early childhood means that increased parental costs extend over many years. Psychoses normally develop in late adolescence or early adulthood, but schizotypal individuals, and juveniles who later develop schizophrenia, exhibit distinctive cognitive profiles, and some features of these profiles can be interpreted to involve reduced demands on parents. We note first that research on the so-called premorbid personality of schizophrenics has focused almost exclusively on identifying cognitive deficits as predictors of later disease development (e.g., Ellison et al. 1998; Sorensen et al. 2006). Moreover, schizophrenia itself, like Kanner autism, involves a considerable degree of pathology, and as such it offers much less-direct insight into the dysregulated adaptive mechanisms that underlie psychosis than does healthy schizotypy (or the equivalents for bipolar disorder and major depression), which exhibits genetic and phenotypic continuity with disorder at one extreme and normality at the other.

Given that psychosis and autism can be characterized as cognitive spectra grading into normality, and that non-clinical ("healthy") individuals with autistic traits and relatively unimpaired individuals on the autistic spectrum exhibit clear patterns of relative cognitive strengths in some aspects of mechanistic and sensory cognition (e.g., Gernsbacher et al. 2006; Mottron et al. 2006; Wheelwright \& BaronCohen 2001), does "healthy schizotypy" also involve specific cognitive strengths (Claridge 1997)? We suggest, based on the overdeveloped mentalistic cognition commonly found in schizophrenia, that healthy schizotypy may often involve enhanced mentalistic abilities and empathy relative to normal individuals (Fig. 4). Enhanced theory of mind in schizotypy is generally consistent with four lines of evidence: (1) associations between positive schizotypy scores and measures of empathy (Dinn et al. 2002; Rim 1994; see also Sullivan \& Allen 1999); (2) better performance by schizophrenic children compared to controls in a task involving deception of others (Pilowsky et al. 2000); (3) more accurate recognition of genuine emotions in paranoid schizophrenia than in normal controls (LaRusso 1978), or in depressed patients (Davis \& Gibson 2000); and (4) enhanced social-emotional creativity and imagination in individuals with increased levels of schizotypal traits (reviewed in Claridge et al. 1990; Nettle 2001). In its usual young-adult study subjects, "healthy" positive schizotypy can also involve higher verbal fluency, "openness" to the environment, and more-developed empathy and altruistic feelings and behavior in the context of spirituality (Fisher et al. 2004; Jackson 1997; Tsakanikos \& Claridge 2005). By contrast, a number of studies have reported reduced or similar levels of empathic perspective-taking, or other theory-of-mind tasks, in schizotypy, schizophrenia, or first-order relatives of schizophrenics (Jahshan \& Sergi 2007; Janssen et al. 2003; Kelemen et al. 2004; Langdon et al. 2006a; Montag et al. 2007; Pickup 2006). Such diversity of results may be related to variation in the tasks, populations, schizotypy criteria utilized, and the positions of subjects on the mentalistic continuum (Fig. 4). An important question for future empirical work is determining the neurological, psychological, and behavioral correlates of theory of mind and empathy enhanced over "normal" levels in non-clinical populations. 
Crespi \& Badcock: Psychosis and autism as diametrical disorders of the social brain

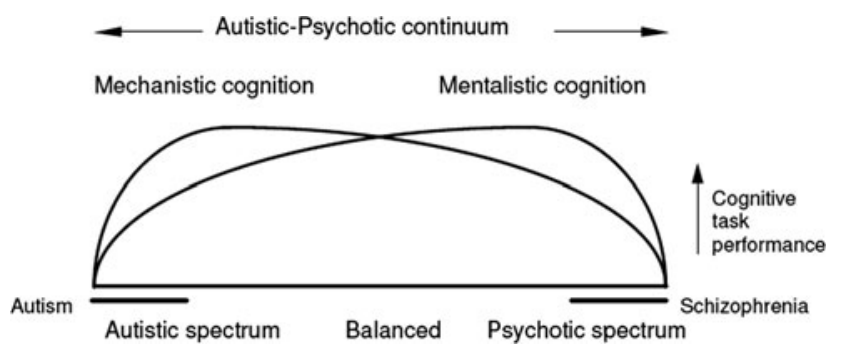

Figure 4. Autistic and psychotic spectrum conditions can be conceptualized as extremes on a continuum of cognitive architecture from mechanistic to mentalistic cognition. The heights of the curves represent relative performance within and between the two cognitive domains, for individuals at any point along the continuum. Autistic-spectrum cognition thus involves enhanced mechanistic cognition but reduced mentalistic skills, while psychotic-spectrum cognition engenders the converse. Schizophrenia is hypothesized to involve notably hyperdeveloped mentalistic cognition, which is associated with a suite of impairments, and autism can be characterized in terms of maladaptively hyper-mechanistic and hypo-mentalistic cognition. The actual shapes of the curves are unknown, but their relative orientation should be roughly as shown.

Positive associations of empathy, theory-of-mind skills, and other traits such as mirror-neuron system function with scores on scales of positive schizotypy in non-clinical populations represent a useful prediction of the hypothesis that the autistic and psychotic spectra represent broadly diametric sets of conditions, with the caveat that existing scales of schizotypy may be geared more towards characterizing impairments than analyzing cognitive-affective architecture.

We thus expect that especially in non-clinical populations of children, mildly above-average levels of positive schizotypy may be associated with easier enculturation (see Brown 2001) and fewer behavioral demands on the mother - traits that should reduce the costs of child rearing. This prediction fits with a central role for the Kevernian maternal brain in the evolution of human cognition and enculturation of offspring (Badcock 2000), and a generally heightened receptivity to the environment in schizotypy and schizophrenia (Claridge 1997; Dykes \& McGhie 1976; Park et al. 1996) compared to avoidance of novel stimuli in autism (Gomot et al. 2006).

An alternative to this "healthy positive schizotypy" hypothesis, which is not mutually exclusive, is that the relatively low birth weight and slow development typical of psychotic-spectrum children are beneficial to mothers (and maternal genes), but the later consequences of such relative energetic benefits in gestation and early childhood may impose costs on both mother and offspring (Fig. 5). By this hypothesis, deleterious behavioral aspects of psychotic-spectrum conditions represent, in part, maladaptive by-products of the tug-of-war between maternal and paternal imprinted-gene effects being disrupted towards a maternal-gene bias. Converse considerations apply to autistic conditions. Disorders of pregnancy provide clear and direct parallels here, in that gestational diabetes and pre-eclampsia, both of which are mediated by maternalfetal conflict, can involve benefits to the offspring (such as higher birth weight) in mild cases, but severe costs to both mother and child when the dysregulated
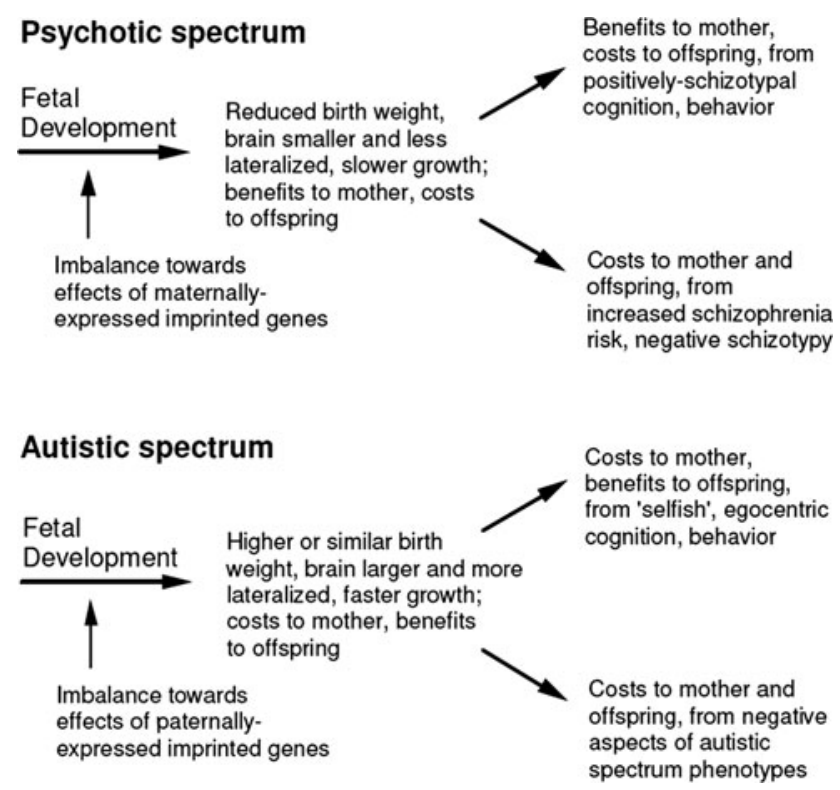

Figure 5. Alternative models for the costs and benefits to mothers and offspring of psychotic-spectrum and autisticspectrum phenotypes can help to explain a range of possible relationships between imprinted gene expression, fetal and child development, and behavior. The psychotic-spectrum case applies most closely to schizotypy and schizophrenia. Here, mothers may benefit from reduced early parental investment, but in later development they may either garnish fitness benefits or suffer costs, depending upon the nature and strength of the effects on offspring cognition and behavior, such as more-pronounced impairments in negative schizotypy. The autistic-spectrum case involves increased costs imposed on mothers in early offspring development; and in later development offspring may either benefit from more-egoistical cognition and behavior, or suffer relative costs, depending on the form and magnitude of the developmental disruptions. All of these costs and benefits should be considered in the context of fitness-mediating interactions between kin.

developmental tug-of-war is more severe (e.g., Catalano \& Kirwan 2001; Haig 1993; Oudejans et al. 2004)

There is currently little directly useful data on the nature of mother-offspring interactions, and the presumably reduced behavioral and energetic costs involved in child rearing, for individuals exhibiting healthy schizotypy. One line of evidence is increased fertility of individuals in one or more category of non-affected first-degree relatives of schizophrenics, which has been reported in multiple studies (Avila et al. 2001; Bassett et al. 1996; Fañanás \& Bertranpetit 1995; Haukka et al. 2003; McGlashen et al. 2006; Srinivasan \& Padmavati 1997; Waddington \& Youssef 1996), although other studies report a lack of such differences (Buck et al. 1975; Rimmer \& Jacobsen 1976). Thus, Fañanás and Bertranpetit (1995) reported that the mothers (but not fathers) of schizophrenics had significantly more siblings than did controls; Bassett et al. (1996) found no significant difference in number of children between siblings of schizophrenics and controls, except for female siblings exhibiting schizotypal traits, who had significantly more offspring; and Waddington and Youssef (1996) reported more siblings for male schizophrenics with a family history of the disorder, compared to those without a family history, and a higher risk of 
Crespi \& Badcock: Psychosis and autism as diametrical disorders of the social brain

schizophrenia in brothers of probands with more than seven siblings compared to fewer than seven. Srinivasan and Padmavati (1997) reported higher numbers of offspring in parents of schizophrenics compared to the general population, and a "non-significant trend" for higher numbers of offspring produced by siblings of schizophrenics, Avila et al. (2001) found a significantly higher number of siblings in schizophrenics, compared to a community sample, and Haukka et al. (2003) reported that sisters of schizophrenics produced significantly more offspring than controls, while brothers produced significantly fewer. Finally, McGlashan et al. (2006) showed with large samples that fertility in parents of individuals with schizophrenia (and with bipolar disorder) was "substantially and significantly" increased. These findings also suggest that effects of increased fertility in relatives of schizophrenics are relatively strong for female, compared to male, relatives (but see also Lane et al. 1995), which fits with schizotypy being more compatible with female than male development and cognition, as described further on. Schizophrenia itself engenders reduced fertility compared to healthy controls, especially for males (Fañanás \& Bertranpetit 1995; Haukka et al. 2003; McGrath et al. 1999), although the presence and strength of this effect varies culturally (Hutchinson et al. 1999).

Some of the epidemiological patterns described above may represent not increased fitness in first-order relatives of schizophrenics, but effects of family size on schizophrenia risk instead. Thus, Wahlbeck et al. (2001b) reported that increased schizophrenia risk involves higher numbers of siblings (controlling for maternal age), and low body mass index at age seven, and Westergaard et al. $(1999 ; 2001)$ found a similar pattern of schizophrenia being more common in larger sibships, with an additional effect from short intervals between siblings. These findings suggest that in some populations, larger families and shorter interbirth intervals engender physiological and metabolic stresses on mothers and offspring that increase schizophrenia risk. This mechanism cannot, however, explain reports of higher fitness in siblings of schizophrenics compared to controls.

Schizotypy may also be linked with other components of reproduction, given that Nettle and Clegg (2006) report an association in a non-clinical population between increased numbers of mating partners and measures of schizotypy. These authors provide evidence that this association is mediated by a positive correlation of the "unusual experiences" dimension of schizotypy with creative activity, and we suggest that enhanced theory-of-mind and language skills in healthy schizotypal individuals (especially relatively creative ones) may facilitate the efficacy of social interactions in determining mating success.

Our hypothesis also predicts that parents with moreautistic offspring should tend to have fewer children due to their increased costs. This prediction appears obvious for cases of Kanner autism due to its high level of impairment at an early age, but cases involving high-functioning autism or Asperger syndrome may provide useful tests. Males also appear to be physiologically more costly to gestate and rear than females (Gibson \& Mace 2003; Rickard et al. 2007; Tamimi et al. 2003), which is consistent with a relatively higher cost of autistic children for mothers, to the extent that such children exhibit relatively male-typical phenotypic traits. Conversely, relatively female phenotypes in children on the psychotic spectrum, as described in more detail later, may make them less costly to rear.

Whether behavioral aspects of the autistic-psychotic spectrum involve trade-offs between mentalistic and mechanistic cognition and abilities remains unclear. Jarrold et al. (2000) found negative correlations, for a non-clinical population and for autistics, between theoryof-mind skills and visual-spatial skills (block design and embedded-figures tests) when verbal mental age was controlled, Johnson and Bouchard (2007) found a negative association between verbal skills and spatial-imagery skills, when the effects of general intelligence were removed, and Nettle (2007) reported a significant negative correlation between Systemizing Quotient scores and Empathizing Quotient scores in a non-clinical population. By contrast, Carroll and Chiew (2006) found that Systemizing Quotient and Empathizing Quotient scores were not significantly related across individuals, with or without controlling for age or verbal ability. In mice, an apparent gain of function mutation in the autism-associated NLGN3 gene is associated with impaired social interaction but enhanced spatial learning ability (Tabuchi et al. 2007), which suggests that such trade-offs may be mediated by single loci and relatively simple mechanisms, such as ratios of excitatory to inhibitory synaptic transmission. The neurocognitive basis for links of mechanistic and spatial skills with relatively "selfish" cognition and behavior in autism also require investigation, although Chen et al. (2007) describe how enhanced spatial skills, and reduced linguistic and mentalistic skills, may be jointly mediated by the egocentric cognition characteristic of autism and Asperger syndrome. Similarly, Langdon et al. (2006a) describe preserved spatial perspectivetaking in autism but impairments in perspectives involving beliefs and intentions.

\section{Sex differences}

If psychosis represents a phenotypic and genomic converse to autism, then it may exhibit a pattern of covariation with sex opposite to the pattern observed in autism. Thus, as males score higher on tests of autistic tendencies (Baron-Cohen 2002; Baron-Cohen et al. 2005), females tend to score higher on standard indexes of schizotypal cognition, which quantify such traits as magical ideation and paranormal or spiritual experiences (Venables \& Bailes 1994; Williams \& Barry 2003; see also Claridge et al. 1997). Females also exhibit increased orienting to gaze cues compared to males (Bayliss et al. 2005), a more highly developed mirror-neuron system (Cheng et al. 2006), and enhanced autobiographic memory (Piefke \& Fink 2005). Positive symptoms are relatively more common in females than males with schizophrenia or schizotypy, and negative symptoms, more frequent in males, are also more recalcitrant to treatment (Caligiuri et al. 2005; Halbreich \& Kahn 2003; Leung \& Chue 2000; Maric et al. 2003; Mata et al. 2000; Moriarty et al. 2001; Räsänen et al. 2000; Salem \& Kring 1998; Sharma et al. 1999; Venables \& Bailes 1994; Williams \& Barry 2003). However, the overall sex ratio in schizophrenia is somewhat biased towards males (Aleman et al. 2003). Whereas autism tends to be much more severe when 
expressed in females (see Badcock \& Crespi 2006; Holtmann et al. 2007), schizophrenia is, on average, considerably more severe on average in males than females (Halbreich \& Kahn 2003; Maric et al. 2003; Mata et al. 2000; Moriarty et al. 2001; Räsänen et al. 2000; Sharma et al. 1999; Walder et al. 2006b; Williams \& Barry 2003).

These diverse findings suggest that sex differences in autism versus schizotypy and schizophrenia mirror some of the differences between males and females. BaronCohen (2003, p. 173) discussed the "extreme female brain" as exhibiting high empathy and low systemizing ability (in contrast to the reverse in autism), but he dismissed its role in psychological disorders on the presumption that hyperdeveloped theory-of-mind skills would be accurate and adaptive rather than pathological.

Sex differences in schizotypy and schizophrenia appear to be related to sex differences in neuroanatomy. Thus, normal females exhibit lower levels of cerebral asymmetry on average than males (Good et al. 2001; Kovalev et al. 2003; Shaywitz et al. 1995; Yücel et al. 2001), especially in language areas (Blanton et al. 2004; Josse \& TzourioMazoyer 2004; Kovalev et al. 2003), a robust pattern that matches the lower anatomical and functional asymmetry in schizotypy and schizophrenia compared to controls. Females also may exhibit a relatively large corpus callosum (for brain size) compared to males (especially in the splenia), although this difference is complex and disputed (e.g., Good et al. 2001; Highley et al. 2003; Mitchell et al. 2003; Schoenemann 2006). Amygdala size is notably associated with sex, psychosis, and autism in the expected pattern. Thus, normal males have a larger amygdala than females (Goldstein et al. 2001b; Good et al. 2001), and controlling for sex, the amygdala is larger in autism and smaller in schizophrenia, as described earlier. Gur et al. (2004) describe how, with regard to orbitofrontal cortex to amygdala ratios, schizophrenic men show "feminization," while women exhibit "masculinization." These patterns are especially telling given the importance of the amygdala and orbitofrontal cortex in functioning of the social brain (Burns 2004; Skuse et al. 2003), and the enhanced abilities of females in interpretation of some social cues, such as those related to fear (McClure et al. 2004). Finally, Mendrek 2007) describes a suite of evidence that schizophrenia engenders a pattern of reversed cerebral sexual dimorphism in structure and function, mainly involving more "female-typical" traits in males; and Nakayama et al. (2007) report significantly higher cortisol levels in males with (female-typical) high scores on the Empathy Quotient test, as well as in females with (male-typical) high scores on the Systemizing Quotient test.

Contrasts have also been documented between some visual-spatial skills (relative to verbal skills) being enhanced in autistic individuals and their first-order relatives (BaronCohen et al. 2001, 2005; Bertone et al. 2005; Bölte \& Poustka 2006), and specifically impaired in psychosis and schizotypy (Kravariti et al. 2006; Langdon \& Coltheart 2001; Toulopoulou et al. 2005), as well as velocardiofacial syndrome (Lajiness-O’Neill et al. 2006; Simon et al. 2002; Zinkstok \& van Amelsvoort 2005) and Prader-Willi syndrome disomy cases (Roof et al. 2000; Whittington \& Holland 2004). Parallel differences in visual-spatial versus verbal skills have been described between normal males and females (Baron-Cohen et al. 2005; Browne 2005; Crow et al. 1998; Gur et al. 2000; Kramer et al. 1996;
Lawrence 2006; Schoenemann 2006; Walder et al. 2006b). Some of these neuroanatomical and cognitive differences may be mediated by differential effects of testosterone and estradiol in fetal development (Lutchmaya et al. 2004; Mendrek 2007). Thus, autistic individuals exhibit a relatively more male-typical pattern of $2: 4$ digit length ratio, but schizophrenics and subjects with schizotypal personality disorder show a relatively more femaletypical pattern (Arató et al. 2004; Manning et al. 2001; 2002; 2004; Milne et al. 2006; Walder et al. 2006a); more-female digit lengths are also associated with higher scores on a depression scale in a non-clinical population (Bailey \& Hurd 2005).

Based on the evidence described above, we hypothesize that a maternal-paternal imprinting axis of cognition, and an axis based on male-female differences, jointly explain key aspects of the sex biases and differences found for autistic- and psychotic-spectrum conditions (Fig. 6). By this model, the two axes overlap partially but broadly with one another, such that the so-called male brain is relatively similar, neuroanatomically and cognitively, to a brain biased towards increased influence of paternally expressed imprinted genes. This overlap explains the male sex-ratio bias and male-typical traits found in autism, and the association between less-extreme impairment and a more male-biased sex ratio (in high-functioning autism and Asperger syndrome). Conversely, a "morefemale" brain is similar to a brain developing under a relatively strong influence of maternally expressed imprinted genes, which explains the female bias in positivesymptom schizotypy and schizophrenia. The most severe neurological and cognitive impairments are found, in both disorders, where the direction of genomic-imprinting dysregulation opposes the sex difference: in females with autism, and in males with schizophrenia. This hypothesis may also help to explain some of the many striking neuroanatomical and other differences between females and males with schizophrenia (e.g., DeLisi et al. 2002; Highley et al. 2003; Troisi et al. 2001; see also Cahill et al. 2004), as well as the more female-like hormonal profiles of male schizophrenics with predominantly negative symptoms (Akhondzadeh et al. 2006; Simpson et al. 2003), a high prevalence of homosexual ideation in male schizophrenics (Planansky \& Johnston 1962), the female-biased sex ratio in major depression (e.g., Piccinelli \& Wilkinson 2000), and the relatively high incidences of psychosis in Klinefelter syndrome (Boks et al. 2007a; DeLisi et al. 2005; van Rijn et al. 2005) and autism in Turner syndrome (Skuse 2005).

\section{Discussion}

Julian Huxley and Ernst Mayr, two architects of the Modern Synthesis of biology, stated in 1964 that "schizophrenia constitutes not a purely medical or psychiatric but a biological problem, with opportunities for a combined attack in many fields - genetics, biochemistry, selection theory, psychology, psychiatry, public health, demography, social science, pathology, and general environmental and reproductive physiology" (Huxley et al. 1964, p. 220). We have tried to marshall such a coordinated attack, on both psychotic and autistic conditions, with evolutionary biology as guide and inspiration, and 
Crespi \& Badcock: Psychosis and autism as diametrical disorders of the social brain

More PATERNAL

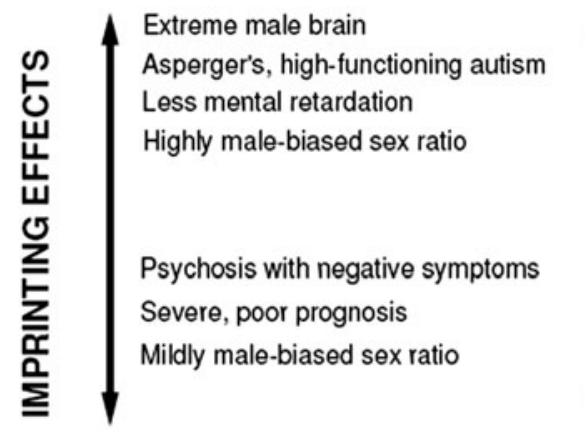

Kanner autism

Severe mental retardation

Rett syndrome

Equal sex ratio

Extreme female brain

Psychosis with positive symptoms

Relatively good outcomes

Female-biased sex ratio

More MATERNAL

More MALE

\section{SEX DIFFERENCES}

\section{More FEMALE}

Figure 6. The interaction of sex differences and genomic-imprinting effects can clarify major features of the autistic and psychotic spectra. The worst impairments in these conditions are found where the direction of sex-difference effects opposes the direction of genomic-imprinting effects: in males with schizophrenia, and females with autism.

genomic imprinting as a simple mechanism that helps to connect genetic and epigenetic variation with cognition.

Our hypothesis can be conceptualized at two interacting levels: (1) the diametric architecture of autistic- and psychotic-spectrum conditions (Badcock 2004), and (2) the underpinnings of this structure in dysregulated genomic imprinting. A diametric structure to autism and schizophrenia has been considered for some traits before: thus, Abu-Akel (1999) and Abu-Akel and Bailey (2000) implied that autism and schizophrenia represent extremes on a continuum of theory-of-mind skills ranging from the deficits commonly found in autism to "hyper-theory of mind" in schizophrenia, C. D. Frith (2004) described "undermentalizing" in autism and "over-mentalizing" in paranoia, and Nettle (2006) anticipated an autism-psychosis spectrum in noting that "autistic traits are in many ways the converse of the unusual experiences component of schizotypy." Similarly, the well-known autistic Donna Williams (1992, p. 204) considered autism and schizophrenia as opposite conditions along a scale of "degree of sensitivity of an automatic cut-off mechanism that stops emotional overload," with the mechanism underdeveloped, oversensitive, and triggered too easily in autism. Most previous research and writing on autism and psychosis has, by contrast, considered the disorders to be etiologically unrelated (or has considered the negative symptoms of schizophrenia in terms of autism), although both disorders are believed to be underlain by dysregulated development of the social brain (Broks 1997; Burns 2004; 2006a; Emery 2000). By our hypothesis, autism and psychosis represent extremes on a continuum of human cognitive architecture from mechanistic to mentalistic cognition, with balanced cognition at the center (Fig. 4). Each set of conditions is extremely heterogeneous but also highly convergent, in that diverse genetic, epigenetic, and environmental effects can generate similar cognitive phenotypes (Badcock \& Crespi 2006; Happé 1994, p. 2; Happé et al. 2006; Keverne 1999; Seeman et al. 2005). These striking convergences are mediated, in our view, by the dynamics of social brain development, with underdevelopment in autistic conditions and hyperdevelopment in psychotic conditions (Badcock 2004), as shown at the cores of Figures 1 and 2. Further tests of this hypothesis should focus on assessing the breadth and depth of diametric phenotypic structure to autistic- and psychotic-spectrum conditions, and testing for trade-offs between mentalistic and mechanistic thought and ability.

A role for genomic imprinting in the diametric structure of autistic and psychotic conditions is based on inclusive fitness theory, which explains how alleles affecting interactions between kin have evolved (Alexander 1979; Hamilton 1964). Inclusive fitness theory provides the evolutionary foundation for understanding animal and human social behavior (Alexander 1979; 1987; 1989), so applying it to understanding disorders of the social brain, the core of human behavior, may lead to useful insights. Such applications are not always straightforward. In particular, developmental systems subject to genomic imprinting effects are expected to resemble dynamic tugs-of-war, whose disruption should lead to relative maternal-gene or paternal-gene benefit only for small deviations, beyond which we expect pathological effects beneficial to no one. Separating adaptation from maladaptation can be extremely difficult for psychological traits (Nesse 2005), and requires knowledge of genetic, epigenetic, and developmental mechanisms (Crespi 2000).

Both non-imprinted and imprinted genes obviously contribute to the neurodevelopmental and physiological processes involved in autistic- and psychotic-spectrum conditions (Crespi, under revision). Our hypothesis should therefore be considered in terms of both genomic imprinting effects on growth, development, cognition, and behavior, and causal factors that are distinct from genomic imprinting but may also include maternal-fetal conflict mediated by non-imprinted genes. The relative contributions of these processes to the etiologies of autistic- and psychotic-spectrum conditions are as yet unclear, but evidence for parent-of-origin effects in these disorders is extensive (Crespi, under revision). Thus, although few of the genes linked so far with autism are known to be imprinted, recent evidence implicates the imprinted gene UBE3A and the imprinting-regulation gene MeCP2 in autism, 
Angelman syndrome, and Rett Syndrome (Samaco et al. 2004; 2005). Similarly, a strong parent-of-origin effect for both schizophrenia risk and relative hand skill has been found for the chromosomal region 2p12-q11 by Francks et al. (2003a; 2003b), who noted that their findings suggest that "lateralized development of the human brain, and complex human cognitive abilities, have been subject to a parental conflict over investment" (Francks et al. 2003b, p. 3227). One of the largest meta-analyses of schizophrenia genome scans to date showed that this region of chromosome 2 exhibited the only linkage signal reaching genomewide significance (Lewis et al. 2003), and Francks et al. (2007) have recently demonstrated the apparent genetic basis of this linkage, in showing that a haplotype of the imprinted, paternally expressed gene LRRTM1 at 2p12 is associated with increased schizophrenia risk and lefthandedness. Our imprinted-brain hypothesis predicts that the risk haplotype should involve lower expression of this gene, or reduced activity of its product, either of which would generate a relative bias towards maternal-gene effects. Further tests of a role for genomic imprinting in the development of autistic and psychotic conditions should focus on identification and functional characterization of brain-expressed imprinted genes, and tests for parent of origin effects on the inheritance of autistic and psychotic phenotypes, especially those that underlie mentalistic skills.

Several important theoretical and clinical implications follow from the conceptualization of a continuum between autistic- and psychotic-spectrum conditions. First, diametric phenotypes in autism and psychosis provide a simple predictive framework for future studies that may reciprocally illuminate the causes and correlates of both sets of conditions. Is the cholinergic system differentially affected in each disorder, given high rates of self-medication via smoking in schizophrenics (De Luca et al. 2004; Ripoll et al. 2004), but apparent low rates of smoking in autistic adults (Bejerot \& Nylander 2003; Lippiello 2006)? Is the wellreplicated lower cancer risk in schizophrenia (Barak et al. 2005; Dalton et al. 2005b; Goldacre et al. 2005; Levav et al. 2007) paralleled by a higher risk in autism (Ingudomnukul et al. 2007)? Do reductions in olfactory and nociceptive sensitivity in schizophrenia and schizotypy (Mohr et al. 2001; Singh et al. 2006) represent a neurologically based contrast with apparently enhanced smell and pain perception in autistic children (Bursch et al. 2004; Cascio et al. 2008; Rogers et al. 2003)? Can autism be characterized as a condition strongly mediated by early childhood overgrowth of the body and brain (Mraz et al. 2007; Sacco et al. 2007), while schizophrenia-risk is associated with childhood undergrowth (Saugstad 1999; Wahlbeck et al. 2001a)? Second, some conditions such as obsessive-compulsive disorder (OCD) and ADHD have been reported as highly comorbid in both autism and schizophrenia (e.g., Bejerot 2007; Hattori et al. 2006; Kayahan et al. 2005; Leyfer et al. 2006; Ross et al. 2006b). By our hypothesis, OCD or ADHD in autistic-spectrum conditions should be fundamentally distinct from OCD or ADHD in psychotic-spectrum conditions (see, e.g., Bürgy 2007; Goos et al. 2007), a prediction that, if supported, strongly impacts on nosology, diagnosis, and treatment. Similar conditions may apply to psychopathy, which has been attributed to subtypes of schizophrenia as well as to Asperger syndrome (e.g., AbuAkel \& Abushua'leh 2004; Blair 2005; Haskins \& Silva 2006). Finally, therapies to reduce hyper-mentalistic cognition in subjects with psychotic-spectrum conditions may be just as useful as the encouragement of mentalistic abilities in autistics, given that impaired theory of mind in schizophrenia is not a deficit so much as a suite of alternative hyperdevelopments (Abu-Akel 1999; Badcock 2004).

As W. D. Hamilton (2005, p. 205) has noted, we live in a world of things and a world of people. An autistic-psychotic continuum can usefully be conceptualized in these overly simplistic terms, although the real world is much more nuanced and complex than our broad, crude strokes can depict. The usefulness of inclusive fitness theory and evolutionary biology in psychiatry, psychology, and neuroscience may ultimately be gauged by the insights that they can provide into the genetic, developmental, and evolutionary bases of the social brain and its disorders.

\section{ACKNOWLEDGMENTS}

Bernard Crespi is grateful to NSERC and the Canada Council for the Arts for financial support. Both authors thank Paul Bloom, Martin Brüne, Will Davies, Clyde Francks, David Haig, Randy Jirtle, Randy Nesse, Daniel Nettle, Sophie van Rijn, and Alfonso Troisi for helpful comments and discussions.

\section{Open Peer Commentary}

\section{Theory of mind in autism, schizophrenia, and in-between}

doi:10.1017/S0140525X08004226

\author{
Ahmad Abu-Akel \\ 8015 Airlane Avenue, Los Angeles, CA 90045. \\ abuakel@hotmail.com
}

Abstract: Autism and schizophrenia are presented as the extremes of disorders affecting the social brain. By viewing human cognition impairment in terms of competence and performance, a variety of social brain disorders can be identified along the autistic-psychotic continuum.

The target article discusses disorders affecting the social brain on "a continuum of human cognitive architecture from mechanistic to mentalistic cognition" (sect. 8, para. 2) in which autism and schizophrenia are the extremes. Within these extremes, there is a variety of conditions that manifest varying degrees of disturbances to the social brain. Theory of mind, which is the ability to represent and apply mental states to both self and other, has become a corollary construct by which we evaluate social cognition in developmental and psychiatric disorders. Theory-of-mind impairment can thus be a result of a disturbance in the ability to represent mental states to self and/or other (i.e., an impairment of competence), as well as in the ability to apply mental states to self and/or other (i.e., an impairment of performance).

By invoking the competence-performance theory of mind paradigm (Abu-Akel 2003), we are now in a position to introduce some of the in-between cases to the extremes proposed by Crespi \& Badcock $(\mathrm{C} \& \mathrm{~B})$. Impairment of competence is associated with individuals with autism who lose the ability to manifest an understanding of both self and other's mental states. Schizophrenic patients with passivity phenomena also fall within this category. These individuals, however, appear only to lose their ability to represent their own mental states (Mlakar et al. 1994). Impairment of performance, on the other hand, is associated with individuals with 
Commentary/Crespi \& Badcock: Psychosis and autism as diametrical disorders of the social brain

delusional and paranoid schizophrenia, negative symptom schizophrenia, and Asperger's syndrome. In the case of paranoid or delusional schizophrenia, we observe theory of mind impairments that result from an over-attribution of mental states to self or others (i.e., hyper-theory of mind) (Abu-Akel \& Bailey 2000). Asperger's syndrome and negative symptom schizophrenia seem to maintain an understanding of mental states, but have a deficit in the ability to manifest this understanding in real-life situations (Bowler 1992). Bowler has shown that although patients with Asperger's syndrome as well as patients with negative symptom schizophrenia perform well on theory-of-mind tasks in laboratory settings, they failed to demonstrate their knowledge of mental states in real life.

The C\&B autistic-psychotic continuum can therefore be further enhanced by incorporating how disorders of the social brain vary along the lines of their ability to represent mental states (i.e., competence) as well as their ability to apply mental states (i.e., performance). It would be intriguing to uncover the extent to which disorders of performance and disorders of competence are etiologically related, within and across groups of disorders, in terms of the role genetic imprinting has in the development of social cognition.

\section{Mapping autism and schizophrenia onto the ontogenesis of social behaviour: A hierarchical-developmental rather than diametrical perspective}

\author{
doi:10.1017/S0140525X08004238
}

\section{Ralf-Peter Behrendt \\ Consultant Psychiatrist, The Retreat Hospital, York, YO10 5BN, United Kingdom. \\ rp.behrendt@btinternet.com}

\begin{abstract}
Co-morbidity of schizophrenia and autism is low because interpersonal concerns of schizophrenic patients presuppose developmental achievements that are absent in autism. Autism may arise if primary anxiety is not overcome at a key developmental stage by affective synchronisation between infant and caregiver. Schizophrenic patients will have learned to regulate primitive anxiety by affectively attuning to narrow social networks but remain highly vulnerable to exclusion from larger groups.
\end{abstract}

Infants are born with a bias to track and fixate face-like stimuli (Johnson et al. 1991; Valenza et al. 1996). Innate perceptual propensities guide learning and ensure that the developing brain receives preferential input from the social environment (Johnson 2001). Newborns are also predisposed to express emotional states by facial expressions and vocalizations (Izard 1994). During short face-to-face interactions, infant and mother attune to each other's affective expressions. Synchronized interactions between maternal and infant affective states are crucial for the infant's social development (Feldman et al. 1999). An inborn tendency to imitate affective expressions enables infants to re-experience (Adolphs et al. 2000) emotional states of caregivers, learn about the association of these states with the satisfaction of needs, and develop conditioned "place preference" to them.

Cerebellar abnormalities in autism are associated with deficits in orienting to visual cues (Harris et al. 1999; Townsend et al. 1999). Deficits in orienting toward human stimuli may represent the primary manifestation of the pathological process in autism (Maestro et al. 2002), causing social deficits by depriving infants of opportunities to learn about the significance of caregiver's emotional expressions. Lack of imitative interaction - possibly secondary to deficits in tendencies to preferentially perceive facial features - contributes to autistic development, as suggested by observations that systematic exposure to imitative interaction later in life can improve social deficits in autism (Escalona et al. 2002; Field et al. 2001).
Given that social recognition depends on oxytocin receptor activation in the medial amygdala (Ferguson et al. 2002), lack of oxytocin or disorder of the amygdala may impair learning of the significance of caregiver's expressions. In autism, oxytocin precursor peptide is processed abnormally (Green et al. 2001), preventing the age-dependent increase in oxytocin plasma concentrations (Modahl et al. 1998). Although some studies report the amygdala is disinhibited or larger in autism, which Crespi \& Badcock $(\mathrm{C} \& \mathrm{~B})$ suggest indicates heightened activation in response to direct gaze - leading in turn to fear and gaze avoidance; others found no amygdala enlargement (Aylward et al. 1999) or enlargement that is proportional to overall increases of cerebral volume (Sparks et al. 2002). It would be premature to generally implicate amygdala hyperactivity in autism, given also that amygdala lesions inflicted in monkeys after birth produce an autism-like pattern of social deficits (Machado \& Bachevalier 2003; Schultz \& Klin 2002).

Infants who develop autism may be less endowed - due to constitutional deficits - with primary anxiety, which expresses itself in distress vocalisations that elicit affective synchronisation with the mother. Autistic individuals were often perceived as placid during infanthood. Primary anxiety goes on to develop into complex social behaviours designed to elicit acceptance and a sense of inclusion from significant others or social groups (see Fig. 1). Midline cerebral cortices, which are implicated in regulation of anxiety, show lower baseline activity in autism and therefore less deactivation during distracting cognitive tasks (Kennedy et al. 2006). If adaptive anxiety-related behaviours fail to achieve their interpersonal or social objective, overt anxiety re-emerges. Positive symptoms in schizophrenia imply increased anxiety; indeed, stronger positive symptoms were associated with increased midline cerebral activity at rest and greater task-induced deactivation (Garrity et al. 2007).

Interpersonal relationships and social networks not only help to overcome primary anxiety, but they also expose to intraspecific aggression, which is woven into social phenomena (Behrendt 2006a; 2006c; Lorenz 1963/2002). For schizophrenics, human relatedness is fraught with terror; they cannot overcome their fear of others because of early-life experiences (Behrendt 2006d; Gabbard 1990). Superego is a metaphor for internalised parental punishment, which importantly contributes to children's socialisation. Schizophrenics show higher sensitivity to threat and punishment (Scholten et al. 2006) and develop a severe and persecutory superego. The clinical picture of schizophrenia "often reflects some of the most brutal morality," attributable to an archaic superego "in which the primal identification (incorporated figure of the mother) holds forth only the promise of condemnation, abandonment and consequent death" (Rosenfeld 1965, p. 66). Cognitive skills based on left frontal cortices are required for socially adaptive expression of intraspecific aggression and affiliative approach, thus balancing fear and withdrawal impulses mediated by the right hemisphere. Relative left-hemisphere dysfunction in schizophrenia may undermine cognitive capacities at the service of social approach functions and engender a predominance of righthemisphere-based social withdrawal functions (Demaree et al. 2005) (see Fig. 1).

Reduction in "mentalistic cognition" ("theory-of-mind" deficit) is not specific to autism (Pennington \& Ozonoff 1996; Rowe et al. 2001) and may not lie at its pathogenetic core. Perception of others' mental states is important in social behaviour, but theory-of-mind skills develop secondarily to infants' automatic tendencies to observe and respond to human affective expressions (Tager-Flusberg et al. 2001). Although others' glances remain insignificant to autistic individuals - and others' stares may continue to be highly aversive - schizophrenics are more concerned about social rejection implicit in others' glances. Their "obsession with the gaze of others as directed toward oneself" may not evidence general "hyperdevelopment of gaze" but manifests deepseated social fears. Schizophrenics' capacity to be "acutely tuned 


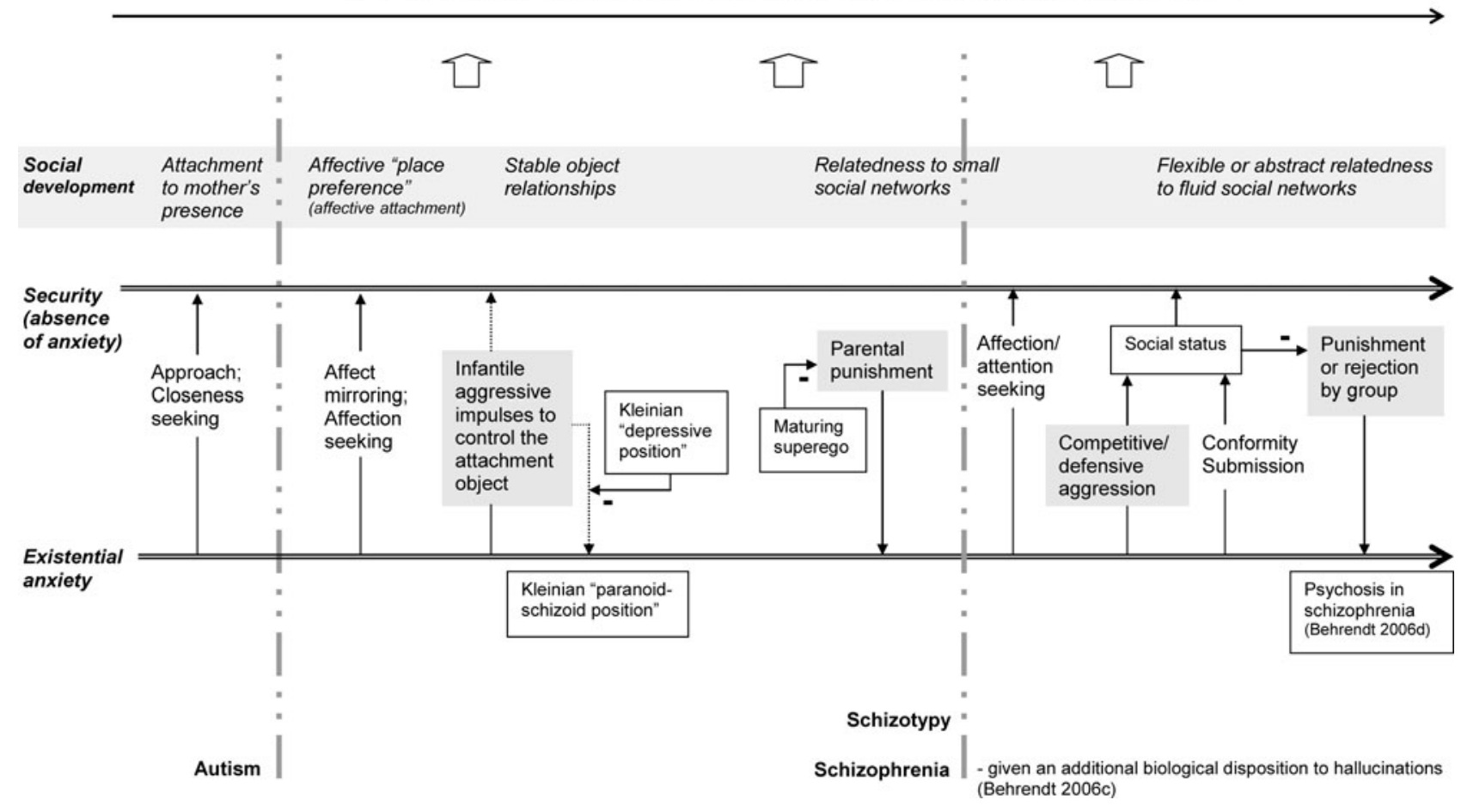

Figure 1 (Behrendt). In the absence of a caregiver or affective synchronisation with the caregiver, existential anxiety manifests in distress vocalisations. Learning to manipulate the caregiver's affective states, thus reducing one's own anxiety, is a foundational step in social development disturbed in autism. Schizophrenics have learned that inclusion in social networks - while potentially exposing them to others' aggression - reduces anxiety; however, underdeveloped social approach behaviour later necessitates psychotic resolution of anxiety. See also Rosenfeld (1965).

to the subtlest unintentional cues" (Nesse 2004, p. 62, cited in the target article) unfolds on a higher developmental level than autistic "literal-mindedness" (sect. 6.3.1).

Persecutory delusions - rather than being instances of "hyper-mentalistic cognition" - can be conceptualised economically as maladaptive defences against these fears. Enhanced amygdala responses to face stimuli (Russell et al. 2007; Surguladze et al. 2006) or verbal hallucinatory experiences in acute paranoid states reflect attention to environmental aspects that remain meaningless to autistic individuals. Increased top-down control and impaired bottom-up processing in cortical language areas (Sumich et al. 2005) mediate attention to hallucinatory voices while disrupting sensory constraints (Behrendt 2006b; 2006e). Attributing verbal hallucinations to "hyperdevelopment in aspects of mentalistic cognition" (target article's Fig. 2 caption) serves those who look for "contrasting patterns of hyperdevelopment and underdevelopment in psychosis and autism" (sect. 6.3.1, para. 4). Cognitivist notions, however, that hallucinations involve "misattribution of agency" or that delusions of control imply an "impaired ability to self-monitor" intended actions (sects. 6.3.1-6.3.3) only confuse matters (Behrendt 1998; 2005).

\section{The "mechanism" of human cognitive variation}

doi:10.1017/S0140525X0800424X

\section{Matthew K. Belmonte}

Department of Human Development, Cornell University, Ithaca, NY 14853-4401.

belmonte@mit.edu

http://www.mit.edu/ belmonte/
Abstract: The theory of psychosis and autism as diametrical disorders offers a tractable and testable view of normal and abnormal human cognitive variation as a function of opposing traits grouped by their selection for maternal and paternal reproductive fitness. The theory could be usefully rooted and developed with reference to the lowerlevel perceptual and attentional phenomena from which social cognitive modules are developmentally refined.

There is a certain mechanistic appeal in theoretical science's types and dimensions of personality. If we can believe in them, such paradigms hold out to us the hope of rendering a welter of human differences comprehensible in terms of cause and effect, and quantifiable in terms of position along some fundamental axis. To the extent that such models are valid, they garner a great deal in terms of predictive, explanatory, or at least descriptive power. To the extent that they are invalid, they lose a great deal in the projection of multifaceted reality onto flat theory. So theoretical abstractions are useful only insofar as they remain grounded in the phenomena that they purport to describe. Crespi \& Badcock $(\mathrm{C} \& \mathrm{~B})$ are keenly aware of this tension between theory and phenomenon, symbol and referent, and this is why they conclude with an apology for "overly simplistic terms" and "broad, crude strokes." Despite this acknowledged coarseness of their model, C\&B have little for which to apologise - for it is the very breadth and force of such strokes that carries science across ideological limits and into new territories.

Like Baron-Cohen's (2002) empathising-systemising duality, which it extends and supplants, this mentalism-mechanism duality has something of an Aristotelian feel. Indeed, it recalls medicine's first explanations of disease in terms of imbalance of the humours. In terms comprehensible to the ancient Greeks we might define the psychotic spectrum as an excess of the mentalistic humour and the autism spectrum as an excess of the mechanistic humour, and observe that these imbalances can be associated with unusual cognitive skills - just as Aristotle characterised what we 
Commentary/Crespi \& Badcock: Psychosis and autism as diametrical disorders of the social brain

now know as mood disorders as an excess of black bile, and observed among his fellow philosophers a certain tendency towards melancholic constitutions (Pies 2007). The difference, of course, is that we don't imagine our humoural abstractions as unitary, physical substances. Rather, we are aware that at genetic and biochemical levels of analysis these mentalistic and mechanistic humours are implemented by dauntingly complex genetic networks which interact with the environment and with each other as development unfolds. This underlying complexity, though, is precisely the condition that makes these insubstantial humours so useful: In parallel with efforts to describe autism and schizophrenia in terms of network interactions among specific genes, we can now describe these conditions in terms of classes of genes united by their selection for maternal and paternal reproductive fitness. Thus, the work of C\&B enables a trait-level analysis of autism and psychosis that complements gene-level analysis.

As $C \& B$ point out, this trait-level view in terms of mentalism and mechanism can in some regards muster greater descriptive and explanatory power than the more immediate view in terms of paternally and maternally imprinted genes themselves, because abnormal biases constructed by such genes can interact with normal programmes and gradients of brain development, spurring knock-on effects as the process of interactive specialisation of neural and cognitive systems unfolds (Johnson et al. 2002; Karmiloff-Smith 2007). Support for the notion of such multi-level cascades of perturbed development comes from studies of schizophrenia demonstrating deficits in early sensory processing (Butler \& Javitt 2005; Butler et al. 2007; Uhlhaas \& Silverstein 2005) and relating auditory frequency discrimination to deficits in affect recognition (Leitman et al. 2007) and visual size discrimination to deficits in theory of mind (Uhlhaas et al. 2006); from studies of autism demonstrating enhanced perception of first-order stimuli (Mottron et al. 2006) and associating theory-of-mind performance with joint attention (Charman 1997) and perceptual disembedding (Jarrold et al. 2000); and from models relating $C \& B$ 's cited variations in brain size to systems-level variation in the balance between short-distance and long-distance connectivities (Lewis \& Elman 2008). In each of these instances, what seems on face an alteration of higher-order cognition is seen to be related to, and in some cases a consequence of, perturbations at lower levels of processing - and in this regard the cited conditions are not disorders of the "social brain" exclusively. Though such relationships may be most obvious in cases of clinical disorders, they seem unlikely to be limited to disordered cognition and instead may extend to normal variation - that is, to the entire continuum of cognitive variation between the mentalistic and mechanistic extremes.

Further refinement will be necessary to explore aspects in which the autism spectrum and the less straightforwardly defined "psychotic spectrum" may have been miscast as opposites. As C\&B note, some empirical aspects are not comparable between these two populations with existing data. Schizophrenia is diagnosed much later than autism, and therefore there remains a dearth of early developmental observations. For example, although the autistic amygdala is indeed abnormally large during childhood and therefore seems a counterpoint to the abnormally small amygdala in schizophrenia, by adulthood the autistic amygdala likewise has become abnormally small (Aylward et al. 1999; Rojas et al. 2004). Likewise, observations of frontal hypoactivation in autism may depend strongly on the behavioural task and on the latency at which activation is measured - in some contexts autistic frontal systems are hyperactivated (Takarae et al. 2007). Regarding sensory filtering, autism shares with schizophrenia a decrease in prepulse inhibition (McAlonan et al. 2002; Perry et al. 2007), and although people with autism do often focus attention very strongly, in some cases maintaining attention is an issue, and many people with autism fulfill criteria for attention-deficit/hyperactivity disorder (Corbett \& Constantine 2006). Familial association of autism and mood disorders (DeLong 2004) calls into question the inclusion of mood disorders within the "psychotic spectrum" in this context. The connectivity issues too remain to be sorted out; both autism and schizophrenia have been described as deficits in brain connectivity (Frith 2005a), but these may be secondary to developmental interactions whose antecedents differ between the two conditions. Despite such areas for refinement, the mentalism-mechanism duality has strong potential to change the way we think about the relation of clinical disorders to normal human cognitive variation, as well as the evolutionary forces that have produced both.

\section{Animal models may help fractionate shared and discrete pathways underpinning schizophrenia and autism}

\author{
doi:10.1017/S0140525X08004251
}

Thomas H. J. Burne, Darryl W. Eyles, and John J. McGrath

Queensland Centre for Mental Health Research, The Queensland Brain

Institute, The University of Queensland, St Lucia, Brisbane, 4072, Australia.

t.burne@uq.edu.au http://www.qbi.uq.edu.au

eyles@uq.edu.au http://www.qbi.uq.edu.au

john_mcgrath@qcmhr.uq.edu.au http://www.qbi.uq.edu.au

Abstract: Crespi \& Badcock (C\&B) present an appealing and parsimonious synthesis arguing that schizophrenia and autism are differentially regulated by maternal versus paternal genomic imprinting, respectively. We argue that animal models related to schizophrenia and autism provide a useful platform to explore the mechanisms outlined by C\&B. We also note that schizophrenia and autism share certain risk factors such as advanced paternal age. Apart from genomic imprinting, copy number variants related to advanced paternal age may also contribute to the differential trajectory of brain development associated with autism and schizophrenia.

Crespi \& Badcock $(\mathrm{C} \& \mathrm{~B})$ suggest that two poorly understood neuropsychiatric syndromes, autism and schizophrenia, can be conceptualized as diametrical, opposed disorders. They suggest the root of the divergence is a disruption of maternally versus paternally imprinted genes, which serves as a switch point, after which the affected individual is shunted down either the schizophrenia or the autism tracks. C\&B array a wide range of evidence to support this ambitious hypothesis. Hypotheses like these are intrinsically appealing - we all desperately wish the complexity of neurobiology could be so easily condensed!

What type of results, from what type of experiment, would allow us to confidently reject the hypothesis outlined by $\mathrm{C} \& \mathrm{~B}$ ? Hypotheses linking early life exposures (genetic and nongenetic) and later neuropsychiatric syndromes are notoriously difficult to test. This is particularly the case for syndromes such as schizophrenia where the disorder may not be apparent until the second or third decade of life (McGrath et al. 2003).

Although clinical research is clearly important, we suggest that animal models remain the only practical tool for unravelling the mechanisms behind early life disruptions that may lead to adult neuropsychiatric disorders. The developing human brain is not open to ready observation, and experimental manipulations of normal brain developmental are clearly neither ethical nor feasible. Rats and mice do not "get" schizophrenia or autism - so animal models will never recapitulate the full phenotype of disorders involving higher cognitive function. However, they provide an experimental platform that allows the researchers to focus on more substrate-pure neurobiological correlates of clinical syndromes (e.g., brain structure or animal behaviour) (Arguello \& Gogos 2006).

Animal studies allow the examination of both candidate genes and environmental exposures on brain development either in vitro or at the whole animal level. In such investigations outcomes as narrow as regional gene expression right up to social behaviour 
Commentary/Crespi \& Badcock: Psychosis and autism as diametrical disorders of the social brain

can be studied. Animal models in schizophrenia research have explored the impact of early life exposure to various candidate risk factors. For example, animal models in schizophrenia research have included postnatal lesioning of selected brain areas (Lipska et al. 1995), prenatal exposure to specific viruses like influenza (Fatemi et al. 2000), and prenatal nutritional deficiencies (Eyles et al. 2003; Kesby et al. 2006). Various animal models of autism are being similarly explored (Crawley 2007; Moy et al. 2007).

To make their case, $\mathrm{C} \& \mathrm{~B}$ have focused on divergence between schizophrenia and autism. We would like to draw attention to two interesting areas of convergence between schizophrenia and autism: namely, prenatal infection, and advanced paternal age. Early life exposure to infection has been associated with an increased risk of autism (Libbey et al. 2005) and schizophrenia (Brown 2006). Prenatal infection models in animals are proving informative for both autism and schizophrenia research (Fatemi et al. 2007; Hornig et al. 1999). The evidence linking prenatal infection has led to rodent models of maternal immune activation. These models, which use non-infective viral (Poly I:C) or bacteriallike components (lipopolysaccharide; LPS) to trigger innate immune systems, have produced informative behavioural phenotypes (Meyer et al. 2006a; 2006b).

Advanced paternal age has also been linked with an increased risk of both schizophrenia and autism. Malaspina et al. (2001) reported that, compared with offspring of fathers younger than age 25 years, the relative risk of schizophrenia was 2.96 in offspring of men aged 50 years or more. Several other groups have replicated this association (El-Saadi et al. 2004; Sipos et al. 2004). It has also been shown that the offspring of men aged 40 or above have a nearly six-fold increase risk of autism spectrum disorders compared to the offspring of men younger than age 30 (Reichenberg et al. 2006). Advanced paternal age may be contributing to the transgenerational accumulation of copy error mutations (Crow 2000; Keller \& Miller 2006). Alternately, epigenetic processes may be compromised in the sperm of older fathers. The resultant alterations in developmental gene structure and/or expression could both contribute to the increased risk of schizophrenia in the offspring of older fathers (Perrin et al. 2007). Once again, studying the impact of advance paternal age on genomic fidelity and structural and behavioural phenotypes is feasible in inbred animal strains.

Many different factors could contribute to the differential trajectory of brain development found in schizophrenia and autism - this is acknowledged by C\&B in their introduction. In the light of this commentary, we would like to propose an alternative hypothesis. Advanced paternal age may lead to both point mutations (Crow 2000) and small de novo chromosomal rearrangements (e.g., copy number variations) and DNA repeat expansions (Pearson et al. 2005; Perrin et al. 2007). There is increasing evidence that many neurological disorders, including autism, are associated with such small genomic rearrangements (Lee \& Lupski 2006; Lupski 2007; Sebat et al. 2007). Indeed, Prader-Willi and Angelman syndromes, which are discussed in detail by $\mathrm{C} \& \mathrm{~B}$, are classic examples of small chromosomal rearrangements (Emanuel \& Saitta 2007). Velocardiofacial syndrome, a deletion syndrome involving Chr 22q11.2, is strongly associated with both schizophrenia and autistic spectrum disorders (Vorstman et al. 2006). Certain types of genomic rearrangements are particularly likely to occur in regions of the genome enriched with short nucleotide repeats (e.g., trinucleotide repeats), expansions of which are already linked to a range of neurological disorders (Nithianantharajah \& Hannan 2007). The fact that disease-associated repeat expansion is a phenomenon that seems unique to humans (Pearson et al. 2005) may appeal to those interested in theories linking human neuropsychiatric disorder to recent evolutionary forces. We propose that the one general mechanism could contribute to susceptibility to both schizophrenia and autism (and probably a wide range of other disorders). However, variations in the location and "dose" of the rearrangements could then be translated to relative brain overgrowth versus undergrowth, as emergent properties of the complex systems governing brain development. These rearrangements may or may not include genomic segments maternally or paternally imprinted.

We hope that the hypothesis outlined by $\mathrm{C} \& \mathrm{~B}$ will stimulate research that links schizophrenia and autism. We argue that animal models provide the most efficient platform to explore the hidden layers of complexity underlying normal and perturbed brain development. In particular, animal models may help fractionate which neurobiological mechanisms are shared between schizophrenia and autism, versus which are discrete for the two disorders.

\section{ACKNOWLEDGMENTS}

We acknowledge the support of the National Health and Medical Research Council of Australia.

\section{Genomic imprinting and disorders of the social brain; shades of grey rather than black and white}

\author{
doi:10.1017/S0140525X08004263
}

William Davies and Anthony R. Isles

Behavioural Genetics Group, Department of Psychological Medicine, School of Medicine and School of Psychology, University of Cardiff, Cardiff CF14 4XN, United Kingdom.

daviesw4@ cardiff.ac.uk

http://www.cardiff.ac.uk/medic/contactsandpeople/d/davies-williamdr-overview_new.html

islesar1@ cardiff.ac.uk

http://www.cardiff.ac.uk/medic/contactsandpeople/i/isles-anthonyroger-overview_new.html

Abstract: Crespi \& Badcock (C\&B) provide a novel hypothesis outlining a role for imprinted genes in mediating brain functions underlying social behaviours. The basic premise is that maternally expressed genes are predicted to promote hypermentalistic behaviours, and paternally expressed genes hypomentalistic behaviours. The authors provide a detailed overview of data supporting their ideas, but as we discuss, caution should be applied in interpreting these data.

Along with their comprehensive review in the target article, Crespi $\&$ Badcock $(\mathrm{C} \& \mathrm{~B})$ present an intriguing, if rather speculative, new hypothesis which attempts to reconcile imprinted gene dysfunction with abnormal brain development and vulnerability to neuropsychiatric disorder. The central tenet of the article is that psychosis and autism represent phenotypes at opposite ends of a neurodevelopmental spectrum, and that the disruption of paternally expressed imprinted genes may predispose to one disorder, whereas the disruption of maternally expressed imprinted genes may predispose to the "opposite" disorder. C\&B's detailed discussion represents an extension of ideas relating to the role that imprinted genes play in social behaviour, which has been alluded to by others previously (Brown \& Consedine 2004; Haig 2000a; Isles \& Holland 2005; Isles et al. 2006). Imprinted genes (i.e., genes that are epigenetically marked such that they are monoallelically expressed in a parent-of-origin dependent manner) are currently an extremely hot topic in behavioural genetics, given their important role in key neurodevelopmental processes (Wilkinson et al. 2007). Thus, this review is timely and provides novel testable ideas regarding the role of these fascinating genes in conferring vulnerability to mental disorder.

To assess the utility of the proposals contained within the target article, it is necessary to answer two pertinent questions: First, can we really say that autism and psychosis are diametrical disorders of the social brain? Second, what is the evidence for (oppositely) imprinted gene dysfunction in these disorders? 
Commentary/Crespi \& Badcock: Psychosis and autism as diametrical disorders of the social brain

On the first point, the authors themselves point out that much of their evidence comes from complex, multigenic disorders where psychosis or autistic traits are endophenotypes. The type of psychosis, neuroanatomy, and cognitive function are likely to be extremely heterogeneous both within, and between, each so-called psychoticspectrum condition, making the grouping of these conditions together as a single entity a potentially hazardous practice. For example, the genetic abnormalities that cause gross developmental abnormalities such as reduced brain size or low birth weight in schizophrenia are likely to be significantly different from those producing a similar outcome in other psychotic-spectrum conditions. Indeed, in some psychotic-spectrum conditions (such as psychotic bipolar disorder), reduced brain size may not be apparent (McDonald et al. 2006), making generalisations about psychotic-spectrum disorders nigh on impossible. It is also relevant to consider the specificity and extent of the "opposite" phenotypes of autism spectrum and psychotic-spectrum disorders. The authors have highlighted a number of endophenotypes that differ between the two types of disorder (bearing in mind the caveat outlined above); however, the two types of disorder share many similarities. For example, dysfunction of attentional and inhibitory processes (both fundamental to normal social functioning) is common to many neuropsychiatric disorders ranging from autism to schizophrenia to bipolar disorder.

In answering the second question posed above, it is necessary to appreciate our current lack of knowledge about imprinted gene function in the brain. The evidence that imprinted gene function may be disrupted in autism and psychosis is largely circumstantial, mainly coming from parent-of-origin dependent linkage/association effects or from studies of subjects with cytogenetic abnormalities in imprinted gene-rich regions. In the former case, candidate genes have often not been identified, or when they have been identified, the molecular basis of the parent-of-origin association effect has not been clarified. Work which includes genetic, epigenetic, functional, and association approaches (such as that by Francks et al. 2007) is likely to improve our knowledge of imprinted gene dysfunction in neuropsychiatric disorder, and will permit us to propose more refined hypotheses. In the latter case, the cytogenetic abnormalities often encompass multiple oppositely imprinted genes, together with non-imprinted bystanders, making drawing conclusions as to the functions of individual imprinted genes complicated.

A number of independent studies have now shown that disruptions of the imprinted gene-rich cluster on human chromosome 15q11-q13 result in mental disorder, including both autism and psychosis. Evidence for this comes from a number of general genomic association studies (Martin \& Ledbetter 2007), and also from the explicit imprinted gene syndromes, Angelman syndrome (AS) and Prader-Willi syndrome (PWS), which display aspects of autistic-like behaviour and psychosis, respectively. Indeed, it is the reciprocity of the neuropsychiatric problems observed in AS and PWS that forms the cornerstone of C\&B's theory, as these disorders are caused by the absence of maternal gene expression, and paternal gene expression at 15q11-q13, respectively. One important consideration, however, is that the original suggestion of such reciprocity (Boer et al. 2002) also identified that it is an overdosage of maternal gene expression found specifically in maternal uniparental disomy and imprinting centre defect sub-types of PWS that predisposes to psychosis. This finding does not contradict C\&B's theory, but it does point to the fact that it is not the lack of paternal gene expression from this cluster per se which results in psychosis. However, these waters are further muddied by the fact that one of the most consistently observed cytogenetic abnormalities in autistic subjects is duplication of the maternally inherited chromosomal region 15q11-q13 (Martin \& Ledbetter 2007); this implies that overdosage of maternally expressed imprinted genes in this region may predispose to autistic tendencies. This observation is inconsistent with the simple idea that over-expression of maternal genes in this region leads to psychosis, but nevertheless may help to explain why a significant proportion of PWS subjects demonstrate symptoms reminiscent of autism spectrum disorders (Dimitropoulos \& Schultz 2007). Thus, the findings from perhaps the best characterised imprinted disorders only partially support C\&B's prediction, in that while the genes in the imprinted cluster on 15q11-q13 are almost certainly involved in social functioning, the functional dissociations between paternally and maternally expressed genes in this region are still unclear.

On a technical note, $C \& B$ state that imprinted genes can be dysregulated in more ways than non-imprinted genes; this is incorrect. However, the monoallelic expression of imprinted genes does render them more vulnerable to complete inactivation. Moreover, given that imprinted genes are important players in fundamental neurodevelopmental processes (Wilkinson et al. 2007), it is likely that the consequences of their dysregulation may be profound. Finally, the complexities of genomic imprinting have necessitated the use of a standardised terminology. For example, it is now common practice to describe imprinted genes on the basis of their expressed allele (i.e., paternally or maternally expressed genes) rather than their silenced allele. As such, ambiguous terms like "paternal-gene bias" need to be clarified to prevent confusion.

In summary, we believe that the ideas presented in the review article are highly original and should be considered when interpreting genetic data from neuropsychiatric conditions. However, the caveats mentioned herein should be borne in mind upon such interpretation.

\section{Heterogeneity and hypothesis testing in neuropsychiatric illness}

\author{
doi:10.1017/S0140525X08004275
}

\section{Curtis K. Deutsch, ${ }^{\text {a }}$ Wesley W. Ludwig, ${ }^{\text {b }}$ and William J. Mcllvane ${ }^{\mathrm{C}}$}

${ }^{a}$ Eunice Kennedy Shriver Center, University of Massachusetts Medical School (UMMS), Waltham, MA 02452, and Psychobiology Program, Harvard Medical

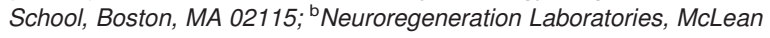
Hospital, Mailman Research Center, Belmont, MA 02478; ' Eunice Kennedy Shriver Center, University of Massachusetts Medical School (UMMS) MRDDRC, Office of the Director, Waltham, MA 02452.

curtis.deutsch@umassmed.edu wludwig@mclean.harvard.edu william.mcilvane@umassmed.edu

Abstract: The confounding effects of heterogeneity in biological psychiatry and psychiatric genetics have been widely discussed in the literature. We suggest an approach in which heterogeneity may be put to use in hypothesis testing, and may find application in evaluation of the Crespi \& Badcock $(\mathrm{C} \& \mathrm{~B})$ imprinting hypothesis. Here we consider three potential sources of etiologic subtypes for analysis.

Coming to grips with the problem of heterogeneity has been a long-standing challenge to neuropsychiatric research. Etiologic and phenotypic forms of heterogeneity have the potential to sap experimental designs of their statistical power to detect group differences among diagnostic and control groups, and to frustrate attempts to identify genes associated with neuropsychiatric diagnostic status (Fanous \& Kendler 2005; McClellan et al. 2007).

Heterogeneity and its consequences hold broad implications for hypothesis testing in the field, including theses put forward by Crespi \& Badcock (C\&B) regarding genomic imprinting. The authors acknowledge that their topics of study - autisticand psychotic-spectrum disorders - have multiple causes that are not necessarily related to imprinting. Nonetheless, they posit a lawful tendency for imprinted genes with maternal expression to underlie the psychosis spectrum, whereas paternally expressed genes contribute to the autism spectrum. Will these effects of imprinting cohere in the face of heterogeneity? 
Commentary/Crespi \& Badcock: Psychosis and autism as diametrical disorders of the social brain

Typically, discussions of heterogeneity in neuropsychiatry strategize to surmount obstacles to discovery. In contrast, our commentary discusses a way that one might turn etiologic heterogeneity to an advantage: for example, finding application in testing the C\&B imprinting hypothesis. We limit our comments to this aspect of the target article, and our examples derive primarily from autism research.

A rigorous test of $\mathrm{C} \& \mathrm{~B}$ 's hypothesis requires multiple instances of imprinting in order to evaluate whether parent-oforigin effects are statistically associated with diagnosis. In the absence of statistical inference, the imprinting hypothesis is speculative and narrowly based. However, combining across a collection of these instances - utilizing an etiologically heterogeneous assortment conditions associated with a diagnostic spectrum - one could determine whether predicted imprinting effects indeed assort with diagnosis. How readily can multiple instances of conditions be attained? We outline three approaches in the following paragraphs.

First, it seems reasonable that known genes for a disorder might provide a test of this hypothesis. However, our current stage of knowledge is limited. Except for syndromic forms in which mutations in a single gene have been specified, no genes have been unambiguously associated with autism (Sebat et al. 2007). Perhaps with the advent of large-scale whole-genome association studies (Ropers 2007), solid findings of single genes may appear for autism and psychosis. Also, the power to detect these genes may be increased by the use of strategies such as stratification on phenotypic subtypes and incorporation of broader phenotypes (Geschwind \& Levitt 2007; Matthysse et al. 1992; McCaffery \& Deutsch 2005; Spence et al. 2006). Once reliable single-gene effects are documented, the imprinted subset would be relevant vis-à-vis the authors' hypothesis.

Second, until these single genes might be found, potentially informative tests may be derived from identified genetic syndromes associated with neuropsychiatric phenotypes. This approach to testing the imprinting hypothesis was adopted by C\&B. Take, for example, the contrast of Prader-Willi and Angelman syndromes - two imprinted forms of a genetic disorder (deletion of 15q11-q13; Christian et al. 1999). The authors adduced a pattern in which autistic symptomatology in Angelman syndrome, as opposed to psychotic features in Prader-Willi syndrome, is associated respectively with dysregulation of imprinted genes towards paternal and maternal origin.

This experimental strategy lends itself to a test of the imprinting hypothesis. Many genetic syndromes display autistic and/or psychotic symptomatology. Are there enough to test the imprinting hypothesis at hand? To address this question, first one might ask whether these syndromes yield bona fide cases of neuropsychiatric illness that meet standard diagnostic criteria. We have found that conditions associated with autism reveal many syndromes that do meet the "gold standard" of the Autism Diagnostic Inventory (revised, ADI-R; Lord et al. 2001), among them: Angelman (Peters et al. 2004), velocardiofacial/diGeorge (deletion of 22q11; Fine et al. 2005), fragile X (Clifford et al. 2007), and Smith-Lemli-Opitz (Tierney et al. 2001), as well as Cowden disease (PTEN mutation; Butler et al. 2005), Mobius sequence (Johansson et al. 2001), neurofibromatosis (Havolvicova et al. 2007), and tuberous sclerosis (Smalley 1998). A test of the $\mathrm{C} \& \mathrm{~B}$ hypothesis would also require a priori identification of imprinting for a genetic marker.

Third, a newly emerging class of genetic markers may prove useful in testing the association of parent-of-origin effects with neuropsychiatric illness. These are alterations in DNA copy number (copy number variants, or CNVs; Iafrate et al. 2004; Sebat et al. 2004), and they are assayed at a level of resolution higher than conventional cytogenetic methods that have been used to identify karyotypic abnormalities in autism (Wassink et al. 2004). Recently, germline mutations have been found to be remarkably prevalent among individuals with autistic-spectrum disorders (Sebat et al. 2007). The genomic regions of these CNVs correlated with autism were highly heterogeneous and included de novo mutations of single genes. In general, variations may be de novo or familial - for example, in which the proband and one parent share the CNV.

The array-based technology to detect these CNVs is now providing yet more resolute assays, and even more CNVs are expected to be identified. Some of these may overlap, deriving from genomic regions that are particularly vulnerable (Perry et al. 2006). Sebat et al. (2007) have already found variants identified by other investigators. Potentially, variants may emerge for autism and psychosis that are tractable to imprinting studies. This subset could be studied in association with neuropsychiatric phenotypes, yielding a quantitative test of assortment.

We have suggested an instance in which heterogeneous genetic markers could be useful in hypothesis testing. Heterogeneity may have other lessons to teach us, as well. How could a miscellany of etiologic factors converge on a common behavioral phenotype? The answer has proven elusive, although there are empirically based models that address the phenomenon. For autism, there have been explanations tied to neuronal connectivity (Just et al. 2004; Rippon et al. 2007), disordered neural excitation or inhibition (Rubenstein \& Merzenich 2003), aberrant connections of neural networks (Geschwind \& Levitt 2007), and neurodevelopmentally altered temporal binding (Brock et al. 2002; Deutsch 1998; Rippon et al. 2007). Understanding how a heterogeneous collection of causal factors emerges as a single diagnostic entity is key in delineating the biology of neuropsychiatric illness.

\section{Is this conjectural phenotypic dichotomy a plausible outcome of genomic imprinting?}

\author{
doi:10.1017/S0140525X08004287
}

\section{Benjamin James Alexander Dickins, ${ }^{a}$ David William Dickins, ${ }^{b}$ and Thomas Edmund Dickins ${ }^{c}$ \\ a505 Wartik Laboratory, Center for Comparative Genomics and Bioinformatics, The Pennsylvania State University, University Park, PA 16802; 'b School of Psychology, University of Liverpool, ERB, Liverpool L69 7ZA, United Kingdom; 'School of Psychology, University of East London, London E15 4LZ, United Kingdom, and Centre for Philosophy of Natural and Social Science, The London School of Economics, London WC2A 2AE, United Kingdom. ben@bx.psu.edu \\ http://www.bendickins.net/ \\ dickins@liverpool.ac.uk \\ http://www.liv.ac.uk/psychology/staff/ddickins.html t.dickins@uel.ac.uk \\ http://www.uel.ac.uk/psychology/staff/tomdickins.htm}

Abstract: What is the status of the dichotomy proposed and the
nosological validity of the contrasting pathologies described in the
target article? How plausibly can dysregulated imprinting explain
the array of features described, compared with other genetic models?
We believe that considering alternative models is more likely to lead in
the long term to the correct classification and explanation of the
component behaviours.

At the conceptual core of Crespi \& Badcock's (C\&B's) case are two developmental syndromes in humans attributed to imprinted genes on chromosome 15q11-13: Angelman syndrome (AS), caused by mutations abolishing expression of the maternally transcribed UBE3A gene (Lalande \& Calciano 2007), and PraderWilli syndrome (PWS), caused by deficits in expression of paternal genes in the same imprinting cluster (Bittel \& Butler 2005). Given this, and the dominance of the conflict theory for the evolution of imprinting (Haig \& Westoby 1989; Moore \& Haig 1991), the effects of intragenomic conflict have been inferred from several phenotypes manifested in these conditions (Brown \& Consedine 2004; Haig \& Wharton 2003). The genetic 
Commentary/Crespi \& Badcock: Psychosis and autism as diametrical disorders of the social brain

causes of autism spectrum disorder (ASD) and schizophrenia are more complex than those of AS and PWS, and manifestly polygenic in nature. This should make one cautious of the authors proposal, though not dismissive.

Because of their complex epigenetic regulation, imprinted genes are vulnerable to dysregulation (target article, sect. 3), though they are not unique in this respect (e.g., maternal behaviour regulates promoter methylation of the glucocorticoid receptor gene in rat pups; Weaver et al. 2004). However, many imprinted genes are expressed in the mammalian brain (Davies et al. 2005), thereby presenting a large mutational target and increasing the prior probability of imprinted gene involvement in ASD and schizophrenia. Classic work with mouse embryos chimeric for wildtype and androgenetic (Ag) or parthenogenetic (Pg) cells (Allen et al. 1995; Keverne et al. 1996) also suggests a role for imprinting in brain development, but some evidence presented by $\mathrm{C} \& \mathrm{~B}$ seems contrary to neuroanatomical predictions one might derive from this work. For example, increased and decreased hippocampal size in autism and schizophrenia (target article, sect. 6.1.2) is not consistent with Pg cell accumulation in the hippocampus (Allen et al. 1995), and overall brain size is decreased in chimeras with a high contribution of $\mathrm{Ag}$ cells (Keverne et al. 1996), contrary to brain size increases in autism (sect. 6.1.1).

These concerns aside, C\&B's theory is impressive in terms of the wealth of phenomena it endeavours to embrace, and several features described in Table 1 are plausibly supportive. Even here, though, the authors' exclusive reliance on the conflict theory may be misleading. For example, in utero growth restriction is associated with paternal over-expression in transient neonatal diabetes (Temple \& Shield 2002), against the predictions of this and some other theories for the evolution of imprinting. Comparisons with existing theories or data are post hoc, and C\&B know they need to propose falsifiable hypotheses. While they make some interesting predictions, we do not believe their model sufficiently specifies how imprinted genes are involved and in what phenotypes.

The behavioural phenotypes of ASD and schizophrenia are complex. In ASD the trio of "impaired social interaction, impaired communication and restricted and repetitive interests and activities" are linked conceptually by jointly providing the inclusive definition of ASD rather than biologically by any strong associations in their occurrence in psychological tests of the general population (Happé et al. 2006) or in genetic twin studies (Ronald et al. 2006). Therefore, comparative studies between groups of ASD versus other individuals could produce artefactual associations between the separate components of this triad (Happé et al. 2006). The same is likely to be true a fortiori for any umbrella concept of schizophrenia (Bentall 2003), let alone for an opposing cluster of psychoticism which also includes bipolar disorder and major depression.

These considerations raise the question, "What constitutes a continuum in biology?" Is a nominal scale sufficient? We see this conspectus of phenotypic features as more idiographic than nomothetic. But even on a nominal scale there is the problem of co-morbidity. The authors cite evidence for co-morbidity of obsessive-compulsive disorder (OCD) and attentiondeficit/hyperactivity disorder (ADHD) with both autism and schizophrenia. From this they predict the existence of different types of OCD and ADHD in autism and schizophrenia. But why do they not consider co-morbidity a major problem for their position? Are there any kinds of co-morbidity that would constitute a disconfirmation of the hypothesis?

Insofar as C\&B's thesis depends on conflict theory, we note the need for hypotheses to be developed regarding differential manipulation of parents in autism and schizophrenia. We remain to be persuaded that the mechanizing/mentalizing dichotomy will map onto more manipulation in autism and less in schizophrenia. It seems reasonable to suppose the existence of Machiavellian manipulators of maternal care possessed of good mentalizing abilities. We must wait for data to be collected to settle this question.
More generally, although we believe that conflict theory has considerable explanatory utility, an alternative model of imprinting evolution under sexually antagonistic selection might help $C \& B$ elucidate the links between parental gene effects and sexual differences (sect. 7). For example, a gene may tend to show expression limited to the paternal allele when alleles of that gene benefit males more than they cost females (Day \& Bonduriansky 2004). Such a mechanism could provide a specific explanation for why autism appears simultaneously to be caused by an excess of paternal gene expression and manifests as an "extreme male brain" phenotype. In this context the authors' observations about the relations between sex and severity in autism and schizophrenia (e.g., their Fig. 6) seem to hint at such a selective regime.

Finally we emphasize the importance of considering alternative genetic models that explain the prevalence of ASD and schizophrenia or their components. We will mention just one here. Some alleles (of one or more genes) may show benefits when inherited alone, but cause mental dysfunction when inherited together such that selection maintains them in the population in a balanced polymorphism. For instance, Nettle and Clegg (2006) have noted that schizotypy is strongly related to creativity, which in turn has been linked to reproductive success (Miller 2001), at least in terms of number of sexual partners over a lifetime. They confirmed this by showing that two out of four component dimensions of schizotypy were positively correlated with mating success in a large sample of British adults that included amateur and professional artists and poets.

\section{Creativity, psychosis, autism, and the social brain}

doi:10.1017/S0140525X08004299

\author{
Michael Fitzgerald and Ziarih Hawi \\ Department of Psychiatry, Trinity College, Dublin 2, Ireland. \\ fitzi@iol.ie \\ http://www.medicine.tcd.ie/psychiatry/ \\ zhhawi@tcd.ie \\ http://www.medicine.tcd.ie/psychiatry/research/neuropsychiatry/
}

\begin{abstract}
In the target article, Crespi \& Badcock (C\&B) propose a novel hypothesis based on observations that a large set of phenotypic traits exhibit diametrically opposite phenotypes in autism-spectrum versus psychotic-spectrum conditions. They propose that development of these conditions is mediated in part by alterations in "genomic imprinting." This hypothesis is based on the model of the Prader-Willi and Angelman syndromes. The authors have produced a masterful discussion of the differences between psychosis and autism. Of course, another article could be written on the similarities.
\end{abstract}

Clinically, the target article of Crespi \& Badcock (C\&B) does have a ring of truth. However, their hypothesis - that the development of autistic- and psychotic-spectrum conditions is mediated in part by alterations in "genomic imprinting" - does not explain or accommodate some potent molecular and genetic considerations. There are two main points to consider:

1. Both of the conditions (autism and psychosis) are highly heterogeneous (as $\mathrm{C} \& \mathrm{~B}$ point out), involving many complex traits. It is unlikely that all these traits are the result of disruption to either maternal or paternal gene imprinting processes, as the number of the imprinted genes is very small and does not account for more than $1 \%$ of the genome at the best estimate. Further, if the Prader-Willi and Angelman syndromes form the central dogma of the hypothesis, then how does a disruption of the imprinting process in the same set of genes result in two conditions with divergent age of onsets - early in the case of autism 
and at adolescence or around the mid-twenties in the case of psychosis?

2. As yet there is no solid molecular evidence (linkage or association) to implicate imprinting or disruption of an imprinting process in either autism or psychosis. If, however, identified in the future, they may have a minor contribution to the whole process of developing the above conditions, as both are polygenic disorders with several genes (each of minor effect) contributing to their development.

C\&B's key table (Table 1 ) is very persuasive in showing the differences between autism and psychosis, and this forms the central hypothesis of the article. However, it is frequently reported in the literature that features of neurodevelopmental disorders are non-specific, and that overlaps in the nosology of neurodevelopmental disorders exist. This is frequently reported in relation to autism and attention-deficit/hyperactivity disorder (ADHD) with attentional difficulties commonly occurring in autism. One of the authors of this commentary (Fitzgerald) has observed an overlap between elective mutism and autismspectrum disorders as suggested in Figure 1 of the target article. Further, individuals with high functioning autism may show fixed and rigid beliefs reminiscent of delusions observed in psychosis. Moreover, creativity is frequently observed in the context of psychosis, particularly in bipolar illness. Although creativity in schizophrenia diminishes after the onset of the condition, family members with less severe traits of the condition can show great creativity, reflecting perhaps broader phenotype features. Asperger's syndrome is also associated with creativity; and although great creativity in old age is uncommon, the lack of decrease of gray matter volume with aging (McAlonan et al. 2002) in persons with Asperger's syndrome may explain the old age creativity sometimes seen with persons with Asperger's syndrome. There are small subgroups of persons with autismspectrum disorders who are fearless. This group has been given insufficient attention in the target article. In addition, all novel stimuli are not avoided in autism, as there is an autistic type of novelty seeking which is partly responsible for their great creativity.

Finally, at times, it appears that the separation between autism and schizophrenia is too neat in C\&B's article.

\section{Private speech, cognitive-computational control, and the autism-psychosis continuum}

doi:10.1017/S0140525X08004305

\section{William Frawley \\ Center for Applied Linguistics, Washington, DC 20016. \\ bfrawley@cal.org}

Abstract: Autism and psychosis manifest private speech disruptions analogous to their diametrical opposition along the autism-psychosis continuum. Autism has naturally suppressed private speech with predictable structural deficits when it does surface; psychosis has overt but ineffectual private speech with similar structural deficits. These private speech oppositions are best understood in the context of the control processes of cognitive-computational architectures.

Crespi \& Badcock's (C\&B’s) autism-psychosis opposition has analogous diametrical differences in the syndromes' self-monitoring and inner private speech. In autism, self-talk for cognitive regulation is absent or reduced, but in psychosis, it is overt, yet ineffectual. These differences gain detail and explanatory power if understood in the context of cognitive-computational control.

Processing architectures distinguish logic (representations per se) from control (management of dataflow) (Frawley 1997; Kowalski 2005a; 2005b; Pylyshyn 1985). Control is clearly seen in cross-domain thinking, either across whole knowledge domains (Carruthers 2002, Frawley 2002b; e.g., coordination of visual and motor representations) or across levels within domains (Frawley 2002a; e.g., at the phonology-syntax interface in language). Inner speech is an instrument of the former and surfaces as private speech to facilitate and regulate cognition and behavior during complex, online thinking. The structure and effectiveness of private speech correlate with task challenge and performance (though, see Fernyhough \& Fradley 2005).

The issue for the autism-psychosis account is exactly how these syndromes and their regulatory disruptions add to the control mix. Self-regulatory talk exhibits two major control failures:

1. Failure to surface at all - or to do so in reduced form. This is a potentially very serious disorder of cognitive control because the lack of self-talk prevents external speech-scaffolding for online thinking.

2. Surfacing with deficits in structure and so functionally ineffective. Such private speech cannot perform appropriate regulatory functions and hence will run parallel to behavior instead of guiding it; it cannot be developmentally internalized (or "go underground," as Vygotsky said); nor can it dynamically manifest itself and re-surface appropriately when it is needed for cognitive and behavioral regulation.

$\mathrm{C} \& \mathrm{~B}$ cite research to the effect that in autism, inner speech is absent or reduced. But under my reading, the autistic individuals in question did indeed have inner speech, but less private speech. Without inner speech, individuals would lack an internal language for cross-domain thinking, and it is not clear that anyone lacks that, whatever the severity of the disorder. Evidence for the existence of inner, but not private, speech in autism can be seen in the results of Williams et al. (2008), who have found that, on a direct phonological encoding task, individuals with autism-spectrum disorder deployed inner speech as much as individuals of comparable mental age without autism.

But reduced private speech is another story. Whitehouse et al. (2006) have found that autistic individuals have truncated articulations in overt labeling tasks. But more curiously, articulatory suppression did not affect autistic individuals' performance in task-switching scenarios (a difficult cognitive requirement), yet it did affect the performance of normal controls. Why?

Normal individuals alternate implicit and explicit self-speech to scaffold behavior under duress. Autistic individuals have inner speech, but are unable to use it overtly in self-guiding tasks. With its repetitive and self-directed behaviors, autism might be expected to encourage private speech. But as a disorder of overactive input filters, autistics use these behaviors to block input, which readily reaches excessive intake in autism. Private speech does not function to block input. So while normal individuals naturally allow inner speech to surface as private speech because they have no blocking problem, autistic individuals naturally suppress it. Additional support for this argument can be found in C\&B's observation that neurological studies of autistics reveal abnormal lack of deactivation of default executive functions when attention is redirected: This suggests that persistence of internal executive control is the normal state for the autistic end of the spectrum when it should otherwise deactivate to refocus attention on new input. Put simply, in autistics, inner speech stays inner. A task which enhances private speech suppression should therefore have no effect on the performance of autistic subjects, as was found, because suppression ratifies the default state of autistic inner speech. It would be surprising if suppression of speech did have an effect on autistic individuals and surprising if it did not for controls.

These findings may in fact relate closely to the severity of the disorder. Low-functioning autistics should have unexpressed inner speech, but high-functioning autistics - those who fall along the cline toward "normal," from Asperger's syndrome to Angelman's syndrome to pervasive developmental disorder not otherwise specified (PDD-NOS) - ought to 
Commentary/Crespi \& Badcock: Psychosis and autism as diametrical disorders of the social brain

have private speech, but it should be functionally defective. I know of no study of this predicted difference, but my informal observations of high-functioning autistics, including my own son, bear this out. PDD-NOS individuals' private speech has deficits in metapragmatics and discourse markers, the very features that index cross-domain control because they encode the line between linguistic and nonlinguistic knowledge. Problems with these structural elements are typical in ineffective private speech, whatever its cause (Frawley 1997).

Thus, as autistic severity lessens and the autistic spectrum approaches normal, private speech should also surface in difficult tasks but be functionally ineffective and have predictable structural deficits. Systematic study of this prediction remains to be done. But the larger point is that the autism spectrum has an analogous private speech spectrum, and the effectuality of private speech might be a new measure of the severity of the autism.

In contrast, psychosis has diametrically opposite private speech manifestations, though with similar functional results. $\mathrm{C} \& \mathrm{~B}$ do not illustrate psychotic private speech, but it is well known that such speech is elaborate and ostensibly social, yet regulatorily ineffective. Jones and Fernyhough (2007) observe that verbal self-mediation in psychosis manifests problems in distinguishing self from other and often takes the form of commands. Psychotic individuals hear their own private speech as orders from others.

As with autistic private speech, problems in psychotic private speech evidence deficits in metapragmatics and discourse forms. Normal private speech metapragmatically signals that the speech is self-derived, as can be seen in the prevalence of self-directed questions ("Now what did I do wrong?"). Moreover, the illocutionary force of normal private speech utterances can be other than a command (directive). Self-directed "Blue, blue" in a task could mean "There's the blue one" (presentative), "I need a blue one" (desiderative), and so forth.

Private speech in individuals with psychosis is overt to match their over-social cognition, but relatively undifferentiated in the way the speech encodes and executes control. As a consequence, this speech for thinking cannot "go underground" to serve inner functions. Just as speech for thinking in severe autism keeps the mind inside itself, so the same speech for thinking in severe psychosis keeps the mind away from itself. A detailed linguistic analysis of the private speech in psychotic syndromes would likely show deficits in the same metapragmatic and discourse markers as in autism, but with different functional effects. A similar diagnostic cline to "normal" should also present itself, with movement from severe to less severe, as measured by private speech and signaled by the gradual reduction and internalization of private speech and concomitant increase of functional cognitive control.

Autism and psychosis are diametric control syndromes - true private speech opposites. Each moves toward normal by the other's failures - autism via increased explicitness and psychosis via increased implicitness.

\section{Imprinting and psychiatric genetics: Beware the diagnostic phenotype}

\section{doi:10.1017/S0140525X08004317}

\section{Lisa M. Goos}

Psychiatry Research, The Hospital for Sick Children, Toronto, Ontario, M5G $1 \times 8$, Canada.

lisa.goos@sickkids.ca

Abstract: Studies of the role of imprinted genes in psychological phenomena are long overdue. The target article is comprehensive, presenting a wealth of important and convergent evidence, and provides an excellent point of departure for further research. However, the authors' evidentiary grasp exceeds the explicatory capacity of the proposed model. Greater genotypic and phenotypic precision would significantly enhance its predictive power.

Imprinted genes expressed in the brain are numerous, and they play important roles in nervous system development (Keverne et al. 1996). Elucidating their influence on psychological function will require greater understanding of epigenetic processes among scientists trained to study behavioural phenomena. For this reason, the comprehensive work presented in Crespi \& Badcock's (C\&B's) article is an important one. The article is exhaustively researched and provides direction for further investigation. Unfortunately, the authors try to explain too much with too little, outstripping the explanatory capability of the model. A wide variety of psychiatric diagnoses are included in the target article, and their dichotomous categorization seems forced at times. Furthermore, the core "balanced see-saw" model presented is insufficiently precise.

Phenotypes in psychiatric research. The target article attempts to integrate a variety of psychological and psychiatric phenomena, many of which have not previously been conceptualized as related. Phenotypic commonalities can be beneficial guides in the search for etiological factors, particularly in the case of complex psychiatric disorders. However, the most narrowly defined traits are likely to be of greatest utility. The broad phenotypes used in target article, alternating between symptoms such as psychosis or depression and diagnostic categories such as autism, schizophrenia, and obsessive-compulsive disorder (OCD), are problematic in this regard.

The power to identify relevant genetic factors is directly influenced by the "purity" of the relationship between the trait under study and the underlying genes. Failure to identify appropriate phenotypes for genetic study is exacerbated in the field of psychiatric genetics where diagnostic classification is often used as the phenotype of interest (Feinstein \& Singh 2007). This is a very serious mistake, as heterogeneity is rampant within diagnostic categories, and individuals with the same diagnosis may vary significantly in phenotype and etiology, even in the presence of high heritability.

Endophenotypes are traits that function as indices of underlying genetic liability to disorder: they are phenotypic manifestations intermediate to the genes and the overt clinical syndrome (Gottesman \& Gould 2003). Consequently, they are more likely to be associated with specific susceptibility genes, and may show higher heritability than the complex clinical endpoints that are the usual focus of psychiatric research.

The use of endophenotypes in the study of complex psychiatric disorders is increasing, and has been shown to facilitate the identification of genetic risk factors (Castellanos \& Tannock 2002; Kruglyak \& Lander 1995). This approach has advanced the study of attention-deficit/hyperactivity disorder (ADHD; Crosbie et al. 2008; Schachar et al. 2005); and evidence that different symptoms in autism are independently influenced by different genes (Ronald et al. 2006; Shao et al. 2003) suggests the same may be true for this complex disorder. To investigate potential imprinting effects, grouping on the basis of behavioural or psychiatric commonalities with known imprinted disorders (such as Prader-Willi syndrome [PWS]), and the study of parent-of-origin effects, are also effective ways to proceed (Goos et al. 2007; Goos \& Ragsdale 2008).

Imprinted genes and social cognition. The authors' imprinted brain model of autism is based on the enhanced influence of paternal genes relative to maternal. The "spectrum" of psychosis is presently suggested as the outcome of increased relative influence of maternal genes over paternal. $\mathrm{C} \& \mathrm{~B}$ posit no significant phenotypic divergence based on the genetic cause of the relative over-expression (i.e., duplication vs. deletion).

This view is consistent with evidence of functional complementarity in a number of imprinted genes, such as insulin-like 
growth factor 2 and its receptor, which form a tightly linked physiological unit (Haig \& Graham 1991). In this case, deletion or duplication of either gene produces predictable outcomes of over- or undergrowth. This logic is unlikely to apply, however, in the case of highly variable behavioural phenomena.

The evolutionary forces driving imprinting (Haig \& Westoby 1989) are compounded and exacerbated by those due to sociality (Haig 2000a). In primates, social skills directly influence both survival and reproductive success (Silk 2007). Furthermore, primate social interactions occur primarily with maternal kin (Cheney et al. 1986). In this context, the adaptive resolution of conflict over resource transfer, for maternal and paternal genes, would involve social skills, in order to maximize resource acquisition, minimize individual risk due to conflict, and restrain direct and indirect maternal fitness costs.

Uniparental cell deposition patterns in chimeric mice support this conjecture. Maternally expressed genes contribute primarily to the development of cortical areas mediating complex socio-cognitive skills, such as memory, planning, and language (Allen et al. 1995). The paternal contribution is primary in the mediobasal forebrain and hypothalamus (Keverne et al. 1996). These areas are crucial for adaptive sociality, largely mediated via oxytocin and vasopressin. These hormones reduce social fear, anxiety, and aggression; moderate stress reactivity; facilitate social memory and behavioural flexibility; and coordinate behavioural and emotional responses to social stimuli (Carter \& Altemus 1997; Kirsch et al. 2005; Winslow \& Insel 2004).

If both maternal and paternal genes were selected to maximize fitness in a social milieu, it is likely that imbalance in either direction would result in social deficits. Moreover, different suites of social impairments may legitimately attract the same diagnosis of autism. Relative paternal over-expression due to mutation(s) in maternally expressed genes could produce the cognitive and language deficits characteristic of autism, as suggested by $\mathrm{C} \& \mathrm{~B}$. However, relative maternal over-expression due to mutation(s) in paternally expressed genes could lead to deficits in socio-emotional processing, such as heightened reactivity, anxiety and fear, and stereotypy and rigidity, also characteristic of autism (Corbett et al. 2006; Hollander et al. 2003; Jansen et al. 2003). Indeed, this postulation is consistent with evidence in the literature documenting cortical or neuroendocrine dysfunction in autism, but not necessarily both (Belmonte \& Carper 2006; Chandana et al. 2005; Green et al. 2001; Jacob et al. 2007; Modahl et al. 1998; Wu et al. 2005).

The target article represents an important step in the integration of genomic imprinting and the study of psychopathology. It would, however, be much more compelling and influential if it were more finely parsed with respect to the imprinted genotypes included in it, as well as the behavioural phenotypes it seeks to explain.

\section{ACKNOWLEDGMENTS}

Dr. Goos is supported by a CIHR Postdoctoral Fellowship from the Ontario Women's Health Council. Thanks to Jennifer Crosbie and Russell Schachar for helpful discussions.

\section{Kinship asymmetries and the divided self}

\section{doi:10.1017/S0140525X08004329}

\section{David Haig}

Department of Organismic and Evolutionary Biology, Harvard University, Cambridge, MA 02138.

\section{dhaig@oeb.harvard.edu}

Abstract: Imprinted genes are predicted to affect interactions among relatives. Therefore, variant alleles at imprinted loci are promising candidates for playing a causal role in disorders of social behavior. The effects of imprinted genes evolved in the context of patterns of asymmetric relatedness that existed within social groups of our ancestors.

Genomic imprinting is an expression of an evolutionary conflict, within the genomes of individual organisms, between genes of maternal and paternal origin. It evolves when a genetically determined action enhances the inclusive fitness of madumnal (maternally derived) genes but reduces the inclusive fitness of padumnal (paternally derived) genes, or vice versa. Such situations arise when the expression of a gene in one individual (the actor) has fitness consequences for other individuals who are asymmetric kin of the actor (Haig 1997). (Asymmetric kin are individuals with different probabilities of carrying copies [identical by recent descent] of an actor's madumnal and padumnal alleles. For example, maternal half-sibs are asymmetric kin, related through the actor's mother but not the actor's father, whereas full-sibs are symmetric kin, equally related to the actor via a shared mother and shared father.)

Offspring are usually symmetric kin of parents (a parent's maternal and paternal alleles each has a $50 \%$ chance of being transmitted to offspring) but parents are asymmetric kin of offspring (an offspring's maternal alleles are present in its mother, but the offspring's paternal alleles are absent from its mother). Therefore, an offspring's interactions with its parents are predicted to be more internally conflicted than are the parents' interactions with the offspring. If one assumes that interactions with mothers have been more salient than interactions with fathers, then paternally expressed genes should promote behaviors that engage maternal attention and elicit maternal care, whereas maternally expressed genes should favor greater independence of the child. This is the principal source of asymmetric kinship considered by Crespi \& Badcock $(\mathrm{C} \& \mathrm{~B})$.

Although an offspring usually has an equal chance of inheriting the madumnal or the padumnal allele at a locus in one of its parents, this symmetry breaks down under inbreeding (Wilkins \& Haig 2003). Offspring are asymmetric kin of their mother if their father is asymmetric kin of the mother (and vice versa). For example, a child conceived by father-daughter incest is not only its mother's offspring but also her paternal half-sib. Similarly, the child of a woman who marries her father's sister's son is simultaneously its mother's offspring and her patrilateral first cousin once-removed. In both cases, the child would be more likely to carry copies of its mother's padumnal alleles than copies of her madumnal alleles. In general, a woman's madumnal alleles are predicted to favor greater aversion for sexual relations with patrilineal kin (Haig 1999a).

Sibling rivalry has probably been intensified in recent human evolution because we have shorter interbirth intervals than our closest relatives but longer periods of juvenile dependence (Kennedy 2005). Therefore, sibs will often have competed for resources provided by adults. Full-sibs are symmetric kin, whereas half-sibs are asymmetric kin. The key question for understanding the role of imprinting in sibling rivalry is the relative importance of competition with paternal half-sibs (patrisibs) and maternal half-sibs (matrisibs). If the variance of reproductive success is higher among males than among females, then the population will contain more patrisibs than matrisibs. However, offspring tend to maintain closer relations with mothers than with fathers. Therefore, interactions are likely to have been more intense with matrisibs (the products of female infidelity and partner change) than with patrisibs. My expectation is that madumnally expressed genes will tend to promote cooperative interactions among sibs whereas padumnally expressed genes will favor competitive interactions.

Asymmetries of relatedness are also present in a child's interactions with its extended family. Grandparents, aunts, uncles, cousins, nieces, and nephews are all, with rare exceptions, asymmetric kin. Imprinted behaviors might play a role in broader family relations if a child's interactions were predominantly 
Commentary/Crespi \& Badcock: Psychosis and autism as diametrical disorders of the social brain

with its mother's kin or its father's kin (or if children evolved specific behaviors for interacting with patrilineal and matrilineal kin). Such asymmetries will have been determined by residence patterns and the stability of pair-bonds (Haig 2000a), whether mothers typically resided with their husband's kin or with their own kin, and whether children followed their mother or stayed with their father after divorce.

I foresee two principal challenges to predicting the effects of imprinted genes on social behaviors from evolutionary first principles. The first challenge (discussed above) will be to understand evolutionarily salient patterns of asymmetric relatedness during human ancestry. The second will be to understand the principal psychological dimensions that have differentially affected matrilineal and patrilineal inclusive fitness, given these asymmetries of relatedness. The latter challenge can be illustrated using the example of a curious "gift" of individuals with Prader-Willi syndrome (PWS). Such individuals perform poorly on most cognitive tests but far outperform individuals from the general population on the assembly of jigsaw puzzles (Dykens 2000). PWS is caused by the absence of expression of genes from padumnal chromosome 15q11-13. This suggests that padumnally expressed genes favor relatively greater development of some psychological attribute that has, as a side-effect, poorer performance on fitting together colored pieces of cardboard. Kinship theory predicts that stronger development of this attribute enhanced individual fitness at a cost to mothers or their families (or reduced individual fitness but conferred a benefit on fathers or their families). The challenge is to understand the nature of the attribute that has been subject to natural selection and how expression of this attribute has differentially affected the fitness of mothers and fathers (or their respective kin).

Both challenges remain to be surmounted in the specific cases of autism and schizophrenia. Infantile onset of autism suggests that the relevant genes may have been subject to selection based on their effects on the mother-infant relationship, whereas the older age of onset of schizophrenia hints that the relevant genes may have been subject to selection for their effects in wider kin networks. The greater challenge is understanding what psychological dimensions have been subject to selection and how variation along these dimensions has affected matrilineal and patrilineal fitness. $\mathrm{C} \& \mathrm{~B}$ suggest that autistic features are more pronounced in Angelman syndrome than in Prader-Willi syndrome, whereas Veltman et al. (2005) conclude the opposite. A detailed study of social behavior in these syndromes is a promising way forward.

\section{Are schizophrenics more religious? Do they have more daughters?}

\section{doi:10.1017/S0140525X08004330}

\section{Satoshi Kanazawa}

Interdisciplinary Institute of Management, London School of Economics and Political Science, Houghton Street, London WC2A 2AE; Department of Psychology, University College London, London WC1E 6BT; and Department of Psychology, Birkbeck College, University of London, London WC1E 7HX, United Kingdom.

S.Kanazawa@Ise.ac.uk

http://www.Ise.ac.uk/collections/MES/people/Kanazawa.htm

\begin{abstract}
Combined with recent evolutionary psychological theories, Crespi \& Badcock's (C\&B's) intragenomic conflict theory of the social brain suggests that schizophrenics are more religious, and autistics are less religious, than the normal population. Combined with the generalized Trivers-Willard hypothesis (gTWH), it suggests that schizophrenics have more daughters, and autistics have more sons, than expected.
\end{abstract}

At the end of his book The Essential Difference, which argues that autism and autistic-spectrum syndrome may result from an "extreme male brain," Simon Baron-Cohen asks, "Is there an extreme female brain?” (Baron-Cohen 2003, pp. 170-85). Crespi \& Badcock $(\mathrm{C} \& \mathrm{~B})$ have now answered this question. Contrary to Baron-Cohen, who speculates that, if there is an extreme female brain, it would not be pathological, $C \& B$ suggest, with a considerable amount of supportive evidence, that an extreme female brain, the polar opposite of autism, may amount to schizophrenia and psychotic-spectrum syndrome.

If $\mathrm{C} \& \mathrm{~B}$ are correct, which all available evidence strongly suggests they may be, then their intragenomic conflict theory of the social brain immediately suggests a couple of novel hypotheses. First, recent evolutionary psychological theories (Atran 2002; Boyer 2001; Guthrie 1993; Kirkpatrick 2005; Miller \& Kanazawa 2007, pp. 158-61) suggest that religion is not an adaptation in itself but a by-product of other psychological adaptations, variously named "agency-detection mechanism" or “animistic bias." We may be evolutionarily designed to infer personal and animistic intentions behind natural phenomena, because the consequence of erroneously overinferring intentions - being paranoid about perfectly natural phenomena - is much less costly in evolutionary terms than the consequence of erroneously underinferring intentions - being killed by predators and enemies when we least expect them (Haselton \& Nettle 2006). We may therefore be designed to be paranoid because it can potentially save our lives, and we may be religious because we are paranoid and see the "hands of God" behind completely natural phenomena.

If this view is correct, then C\&B's theory suggests that schizophrenics, who are hypermentalistic and are more "paranoid," may be predisposed to be more religious, more prone to see the hands of God behind natural phenomena, just as some (McNamara 2001; Miller \& Kanazawa 2007, p. 206, n13) suggest that autistics should be less religious because of their hypomentalism (absence of theory of mind). C\&B identify "overestimation of meaningfulness of naturally occurring coincidences" (sect. 6.1.3, para. 2) as one of the symptoms of schizophrenia.

In virtually all nations of the world, women are more religious than men. While Alan S. Miller and I (Miller \& Kanazawa 2007, pp. 161-65; cf. Miller \& Stark 2002) explain this in terms of women's greater tendency toward risk aversion, C\&B's theory suggests another explanation: If religion is mentalizing natural phenomena, and if the female brain tends toward mentalizing, then women should naturally be more religious.

Second, in an entirely different area, the generalized TriversWillard hypothesis (gTWH) (Kanazawa 2005) proposes that parents who possess any heritable trait which increases male reproductive success at a greater rate (or decreases male reproductive success at a smaller rate) than female reproductive success in a given environment, will have higher-than-expected offspring sex ratios (more sons). Conversely, parents who possess any heritable trait which increases female reproductive success at a greater rate (or decreases female reproductive success at a smaller rate) than male reproductive success in a given environment, will have lower-than-expected offspring sex ratios (more daughters). Because body size and tendency toward violence are distinct advantages in male intrasexual competition for mates and status, big and tall parents are more likely to have sons (Kanazawa 2005; 2007b), and violent men are more likely to have sons (Kanazawa 2006). Because physical attractiveness, while an advantage for both men and women, is even a greater advantage for women than for men, more beautiful parents are more likely to have daughters (Kanazawa 2007a). Because language and communication are more important to women, and thus language impairment is relatively more costly for them, mothers (though not fathers) with a developmental language impairment have more sons than daughters (Tallal et al. 1989).

I have applied the gTWH to brain types and shown that those with "strong male brains," such as engineers, mathematicians, 
Commentary/Crespi \& Badcock: Psychosis and autism as diametrical disorders of the social brain

and scientists, are more likely to have sons, because the male brain is more adaptive for boys. In contrast, those with "strong female brains," such as nurses, social workers, and kindergarten teachers, are more likely to have daughters, because the female brain is more adaptive for girls (Kanazawa \& Vandermassen 2005). If autism and autistic-spectrum syndrome are the result of "extreme male brains," as Baron-Cohen (2003) suggests, then autistics should have more sons. Conversely, if schizophrenia and psychotic-spectrum syndrome are the result of "extreme female brains," or hyper-mentalism, as C\&B propose, then they should have more daughters. Although $\mathrm{C} \& \mathrm{~B}$ cite a large number of studies to demonstrate that first-degree relatives of schizophrenics have more children (sect. 6.4), they do not reveal (nor do I know) whether schizophrenics and their relatives have lower offspring sex ratios (more daughters). This prediction assumes that the genetic tendency toward schizophrenia is heritable, which is reasonable, given that the genetic tendency toward autism appears heritable (Baron-Cohen et al. 1998).

There is one potential confound, however. If lighter birth weight and slower postnatal growth of schizophrenics, and heavier birth weight and faster postnatal growth of autistics, which $\mathrm{C} \& \mathrm{~B}$ document, continue throughout development, then autistics on average should have larger adult stature than schizophrenics. If this is the case, then autistics should be expected to have more sons, and schizophrenics more daughters, purely because of their body size (Kanazawa 2005; 2007b). It would therefore be necessary to control for body size in order to determine the partial effect of autism and schizophrenia on offspring sex ratios. While, given their low frequency in the population, it would be difficult to detect the effect of autism and schizophrenia on offspring sex ratios in representative samples, one should be able to determine, with clinical samples of autistics and schizophrenics with children, whether their offspring sex ratios significantly deviate from the normal secondary sex ratio of .5122 (105 boys for 100 girls).

\section{Problems with the imprinting hypothesis of schizophrenia and autism}

doi:10.1017/S0140525X08004342

\section{Matthew C. Keller \\ Department of Psychology and Institute for Behavioral Genetics, University of Colorado at Boulder, Boulder, CO 80309.}

matthew.c.keller@gmail.com www.matthewckeller.com

Abstract: Crespi \& Badcock $(\mathrm{C} \& \mathrm{~B})$ convincingly argue that autism and schizophrenia are diametric malfunctions of the social brain, but their core imprinting hypothesis is less persuasive. Much of the evidence they cite is unrelated to their hypothesis, is selective, or is overstated; their hypothesis lacks a clearly explained mechanism; and it is unclear how their explanation fits in with known aspects of the disorders.

Crespi \& Badcock $(\mathrm{C} \& \mathrm{~B})$ have done a wonderful job synthesizing a prodigious amount of information on schizophrenia and autism. In doing so, they have made a convincing case that these disorders are diametric malfunctions of the social brain. On a surprisingly large number of anatomical and cognitive dimensions, schizophrenia (or at least its positive symptoms; negative symptoms hold an awkward place in the target article) and autism appear to be diametrically opposed. Unfortunately, the evidence $\mathrm{C} \& \mathrm{~B}$ bring to bear on their central imprinting hypothesis is less convincing. In the following paragraphs, I expand upon this and other reservations.

Much of the evidence C\&B discuss is unrelated to their core hypothesis. C\&B's core hypothesis is that if gene expression is paternally biased, offspring should attempt to extract more maternal resources (increasing autism risk as a side effect) than if gene expression is maternally biased (increasing schizophrenia risk). However, the bulk of C\&B's evidence (lateralization, hemispheric dysfunction, mirror neuron dysregulation), and especially their behavioral evidence (gaze sensitivity, delusions, theory of mind, etc.) has little to do with the offspring's extraction of maternal resources. Possibly a mentalistic cognitive style (related to schizophrenia) could make a child easier to raise, but it also could easily do the opposite by enabling the child to be better at playing social chess. $C \& B$ state that autistic traits appear to impose fitness costs to mothers, but so too would traits related to virtually any childhood-onset psychiatric disorder. The evidence $\mathrm{C} \& \mathrm{~B}$ present favoring schizophrenic/schizotypic behavioral traits being less costly to mothers has to do with fertility of schizophrenic relatives (of questionable validity; see further on). But this is a red herring: Of relevance to their hypothesis is whether schizotypic traits reduce maternal investment, not whether they increase fertility.

Similarly, C\&B conflate two very different issues: maleness versus femaleness, on the one hand, and paternal versus maternal gene expression, on the other. Much of C\&B's evidence (Table 1 of the target article) seems more directly related to extreme male versus female brains, rather than to extreme paternal versus maternal gene expression. $\mathrm{C} \& \mathrm{~B}$ appreciate this but seem to hedge: "By this model, the two axes overlap partially but broadly with one another, such that the so-called male brain is relatively similar ... to a brain biased towards increased influence of paternally expressed imprinted genes" (sect. 7, para. 5). No explanation is provided for this striking statement, despite the fact that it is not obvious why these two axes would be related. Paternally expressed genes are expressed in both male and female offspring, and to my knowledge have no inherent relationship to maleness versus femaleness. By analogy, it would be wrong to assume that IGF-II protein levels are higher in males than females simply because the Igf2 gene is paternally active (indeed, its levels are the same in males and females; Juul et al. 1995). For the same reason, it is wrong for $C \& B$ to presume that a maleness-autism association is consistent with increased paternally expressed genes, or vice versa for a femaleness-schizophrenia association.

C\&B's core hypothesis lacks a clearly explained mechanism. $\mathrm{C} \& \mathrm{~B}$ do not provide a clear explanation for why the putative imbalance in paternally and maternally gene expression occurs. Other disorders related to such an imbalance (e.g., Prader-Willi and Angelman syndromes) occur due to deletions, duplications, or uniparental disomy of specific chromosomal areas - but these causes are rare, and the disorders caused by them are commensurately rare (about 1 in 20,000). Schizophrenia and autism are, on the other hand, 50 (autism) to 200 (schizophrenia) times more common than either Prader-Willi or Angelman syndrome (Jablensky et al. 1992; Rutter 2005). What specific mechanism is being proposed that causes an imbalance in imprinted gene expression, and why does the imbalance occur so often? Moreover, what explains the heritability of autism and schizophrenia? Without a clear description of the mechanism that causes the putative imbalance, these questions can be answered only vaguely, making it difficult to derive quantifiable predictions from the hypothesis.

C\&B fail to discuss counter-evidence and overstate supportive evidence. First, $\mathrm{C} \& \mathrm{~B}$ state "imprinted genes comprise only about $1 \%$ of the genome," (sect. 3, para. 2) but in fact the percentage is much smaller. $\mathrm{C} \& \mathrm{~B}$ presumably intended to say $1 \%$ of genes rather than $1 \%$ of the entire genome. However, even this $1 \%$ figure (around 250 genes) would be too high - 60 to 80 genes are known to be imprinted in humans (Imprinted Gene Catalog; http://igc.otago.ac.nz/home.html). Given that only a subset of these 60-80 genes is likely to be involved in autism and schizophrenia risk, it is, again, difficult to see how imprinting in such a small subset of genes could malfunction often enough to produce the rates and heritabilities observed for the two disorders. 
Commentary/Crespi \& Badcock: Psychosis and autism as diametrical disorders of the social brain

Second, the most direct prediction from C\&B's hypothesis is that imprinted genes are over-represented among genes affecting schizophrenia and autism risk. Evidence is available but was only selectively discussed by $\mathrm{C} \& \mathrm{~B}$. Imprinted genes tend to occur in clusters; over half of them (47) are found in just three regions: $7 q 32,11 p 15$, and 14q32. However, the same meta-analysis discussed by C\&B (Lewis et al. 2003) did not find any of these regions to be among the top 19 most likely schizophrenia gene regions (see Lewis et al. 2003, Table 2). On the other hand, data indicating likely linkage peaks near $7 q 32$ for autism (Badner \& Gershon 2002) seems to support C\&B's hypothesis with respect to autism.

Third, C\&B overstate the evidence for increased fertility among relatives of schizophrenics. $C \& B$ note that "one or more category" of first-degree relatives have higher fertility (sect. 6.4), but such "supportive" evidence includes, for example, the fertility of sisters but not brothers - even when overall sisterplus-brother fertility is lower than the general population (Haukka et al. 2003). Such selective presentation of the data is frustrating. If one studied any group (e.g., men with arthritis) and conditioned on enough relative classes (e.g., number of maternal siblings, cf. Fañanás \& Bertranpetit 1995), significant fertility differences could probably be found somewhere. A more balanced presentation of the data would show that siblings and offspring of schizophrenics have equal or lower fertility compared to the general population (Bassett et al. 1996; Haukka et al. 2003; McGlashan et al. 2006; McGrath et al. 1999; Rimmer \& Jacobsen 1976; Srinivasan \& Padmavati 1997; Svensson et al. 2007), whereas parents of schizophrenics have equal or higher fertility (Avila et al. 2001; McGlashan et al. 2006; Srinivasan \& Padmavati 1997; Svensson et al. 2007; Waddington \& Youssef 1996). As noted by $C \& B$, data on higher parental fertility is ambiguous because it could simply indicate that lower parental investment (pre- or postnatally), which must occur in larger sibships, is a schizophrenia risk factor.

How does evidence for a mutational role in schizophrenia and autism risk fit in with C\&B's hypothesis? C\&B ignore the compelling and growing evidence that both new and circulating deleterious mutations play an important role in autism and schizophrenia risk (reviewed in Keller \& Miller 2006; McClellan et al. 2007). Indirect evidence includes: (a) the apparent fitness costs of autism and schizophrenia; (b) the likely effect sizes of susceptibility alleles; and (c) the increased risks of schizophrenia and/or autism with brain trauma, inbreeding, paternal age, and ionizing radiation. More direct evidence based on DNA sequencing or copy number detection is increasingly available and also supports a mutational role (Cheng \& Chen 2007; Durand et al. 2007; Lencz et al. 2007; Sebat et al. 2007; Sutcliffe et al. 2005). It would be useful for $\mathrm{C} \& \mathrm{~B}$ to clarify whether they consider mutations as a contributing cause of the imbalance itself, or an unrelated and additional mechanism (one of the "myriad causes," sect. 1), or inconsistent with their hypothesis.

\section{Hypo- or hyper-mentalizing: It all depends upon what one means by "mentalizing"}

doi:10.1017/S0140525X08004354

Robyn Langdon and Jon Brock
Macquarie Centre for Cognitive Science, Macquarie University, Sydney,
New South Wales 2109, Australia.
rlangdon @maccs.mq.edu.au
http://www.maccs.mq.edu.au/members/profile.htm?memberID=60
jbrock@ maccs.mq.edu.au
http://www.maccs.mq.edu.au/members/profile.htm?memberID=203

Abstract: By conceiving of autism and psychosis as diametrically opposite phenotypes of underactive and overactive mentalizing, respectively, Crespi \& Badcock $(\mathrm{C} \& \mathrm{~B})$ commit themselves to a continuum view of intercorrelated mentalizing functions. This view fails to acknowledge dissociations between mentalizing functions and that psychotic people show a mixture of both hypo- and hyper-mentalizing.

Crespi \& Badcock $(\mathrm{C} \& \mathrm{~B})$ envisage a continuum of mentalistic cognition, affect, and behaviour with normality at the centre and autism and psychosis at opposite dysfunctional poles: Autism reflects a bias toward paternally expressed genes and hypo-mentalizing; psychosis reflects a bias toward maternally expressed genes and hyper-mentalizing. This commentary is restricted primarily to the cognitive level of this model.

The implication of C\&B's model is that capacities for a suite of mentalizing functions all vary roughly in line with each other as one moves along the continuum. This conception is at odds with findings of dissociations between mentalizing functions. Psychopaths, for example, show good cognitive mentalizing (they understand other people's thoughts well enough to adeptly manipulate and deceive those others) alongside a lack of empathy (i.e., poor affective mentalizing: Blair 2005). Of more direct relevance to C\&B's model, psychotic people report the opposite pattern: a difficulty with understanding other people's thoughts (i.e., poor cognitive mentalizing) alongside intact affective empathy (Montag et al. 2007). To treat mentalizing as a unidimensional continuum of intercorrelated functions is an oversimplification.

It therefore becomes important to focus on more specific socialcognitive domains: processing of eye-gaze information, using mentalistic language to communicate about minds, theory of mind (ToM), and so forth. Within each domain, the strongest test of $\mathrm{C} \& \mathrm{~B}$ 's proposal is to compare data collected from autistic and psychotic individuals using similar paradigms. To illustrate, Langdon et al. (2006b) used cues of other people's gaze-direction to orient subjects' own attentional shifts and found evidence that automatic orienting to gaze might be heightened in psychotic people. Autism studies of this type are more common, but the findings are mixed. Ristic et al. (2003) found an absence of rapid, automatic orienting to static eye-gaze cues in autistic subjects - broadly the opposite of Langdon et al.'s findings. However, two further studies using procedures more comparable to the Langdon study reported normal reflexive orienting to eye-gaze in autism (Senju et al. 2004; Swettenham et al. 2003). While aspects of processing eye-gaze information are clearly impaired in autism, it seems unreasonable to summarily describe autism and psychosis as polar opposites.

Direct comparisons can also be made between studies in which participants described interactions between animated geometric shapes. Several studies have reported a reduced usage of mentalistic language (e.g., the large triangle is encouraging the small triangle) by autistic individuals when describing "ToM scenes" of complex social interactions (Castelli et al. 2002; Klin \& Jones 2006; although see Abell et al. 2000). While autistic individuals under-mentalize, Russell et al. (2006) found that paranoid psychotic patients over-mentalize in this paradigm, ascribing intentions to random movements designed as non-ToM control stimuli. Crucially, however, these same patients also under-mentalized in the ToM condition; just like the autistic individuals, they used significantly less mentalistic language than controls to describe animations of socially complex behaviours.

Likewise, Langdon (2005) found that schizophrenic patients evidenced both under- and over-mentalizing within the same joke-appreciation task. When presented with ToM cartoons that required accurate mental-state inferences in order to get the joke, these patients used less mental-state language than healthy controls and acted as if completely insensitive to the likely contents of the cartoon characters' minds. Yet these same individuals also over-mentalized in a non-mentalistic control condition where they ascribed negative intentions to innocent cartoon characters.

A "hyper-mentalizing" account seems inadequate here. Individuals with psychosis do over-attribute intentions in some contexts, but in others they under-mentalize. One argument might 
Commentary/Crespi \& Badcock: Psychosis and autism as diametrical disorders of the social brain

be that stimuli used in ToM tasks activate too many mental-state representations in psychotic people. This would explain the overuse but not the underuse of mentalistic language. Arguably a greater interference between competing mental-state representations might leave patients more confused in ToM conditions. But why should this lead to less mentalistic language, and what causes the greater interference anyway?

An alternative conceptualisation of hyper-mentalizing relates to the inappropriate attribution of self-knowledge to other people. This could explain why psychotic individuals fail to infer those more complex mental states like trickery that typically feature in ToM conditions, described above, as well as in more classic ToM tasks. This argument, however, appears incompatible with the persecutory-deluded people's common complaints that other people do not truly understand them. Why so, if they over-attribute to others what they themselves know? Moreover, in both autism and psychosis, there seems a difficulty with setting aside self-knowledge so as to appreciate another person's unique subjective world. This is the very essence of ToM, so what does it mean to say that one is hypo while the other is hyper?

An alternative viewpoint is provided by Langdon and colleagues (Langdon 2003; Langdon \& Coltheart 2001; Langdon et al. 2001). They propose that poor ToM task performance in schizophrenia reflects impaired perspective-taking (i.e., a difficulty with entertaining multiple perspectives of the same here-and-now reality). Impaired perspective-taking explains the egocentric projection of psychotic people's own suspicions and biases onto innocent others and ambiguous situations (over-mentalizing) as well as their failure to grasp the interplay between different perspectives in more complex social interactions (under-mentalizing).

In sum, any theory contrasting autism and psychosis must take account of comparable studies of the two disorders rather than relying on general descriptions. Few such comparisons have been conducted, but when relevant data exist, they point to similarities as well as differences. Therefore, regardless of any progress at the genetic or neurological level, we suggest that C\&B's mentalizing spectrum must be better specified cognitively. There are differences, for example, among the following:

The capacity to represent "decoupled" mental states (as about yet separate from the world)

The ability to respond to and interpret eye-gaze cues

The tendency to ascribe psychological causation to random or ambiguous stimuli

The ability to appreciate other people's likely thoughts in light of their circumstances (i.e., perspective taking)

Some of these faculties are intact in psychotic people but some are impaired. For some faculties, it might be possible to conceive of a continuum with pathological hypo- and hyper-abilities at opposite poles. For others, it seems unclear how hyper-abilities might be manifested.

\section{Why is creativity attractive in a potential mate?}

\author{
doi:10.1017/S0140525X08004366
}

\section{Daniel Nettle}

Centre for Behaviour and Evolution, Newcastle University, Newcastle NE2 4HH, United Kingdom.

\section{Daniel.nettle@ncl.ac.uk www.danielnettle.info}

Abstract: A number of studies suggest that women find artistically creative men attractive, especially in the short-term mating context. Artistic creativity (but not mathematical or technical creativity) is linked to psychosis-proneness. I hypothesise that in preferring artistically creative men, women may be choosing paternal genotypes that make babies that are not excessively somatically demanding on them.
Crespi \& Badcock's (C\&B's) magisterial article sets up a host of important and novel hypotheses about the phenotypes of psychosis and autism, the genetic and developmental mechanisms underlying these pathologies, normal variation in milder psychosis-like and autistic-like traits, and sex and parent-of-origin differences in all of the above. Here, I wish to focus briefly on possible implications of their thesis for one related area: the role of artistic creativity in male sexual attractiveness.

A number of lines of evidence suggest that artistic creativity is found sexually attractive. Nettle and Clegg (2006) found that poets and artists had more sexual partners than community controls, a finding repeated for males in a different population of visual artists in Clegg's (2006) doctoral study, which additionally showed that the increased number of partners stemmed from a more short-term mating strategy. Haselton and Miller (2006) devised vignettes of poor but creative versus rich but uncreative men. Women in the fertile phase of their menstrual cycle shifted preference towards the poor but creative man for a potential short-term mate (long-term preferences were unaffected). Such menstrual cycle shifts are thought to maximise genetic quality at the expense of, say, propensity to provide parental investment. Finally, Griskevicius et al. (2006) found that whenever men were given cues designed to activate mating goals, they displayed more creativity in a number of tasks. This suggests a male response to general female interest in male creativity as a mate-selection indicator.

A standard account of these findings might be the following: Creativity is closely linked to general intelligence, and general intelligence is a consequence of the overall functional efficiency of the nervous system. This in turn will be affected by numerous developmental factors, including genetic mutational load. The substantial correlation of measured general intelligence to physical symmetry, long used by biologists as a marker of developmental quality, supports the relationship of general intelligence to mutational load (Prokosch et al. 2005). Thus, creativity is signalling intelligence which is signalling good overall genetic quality (Haselton \& Miller 2006; Miller 2000).

This account appears plausible enough, but there is an unresolved issue. Why is it particularly artistic-type creativity - namely, remote verbal and visual associations - which is found to be attractive? Mathematical, technical, or engineering ability could equally well signal high intelligence, and indeed, these kinds of capacities would have been very useful for survival in the ancestral environment. It is here that the connection to the autism-psychosis spectrum comes in. Artistically creative individuals tend to score highly on measures of psychosis-proneness, while mathematicians have unusually low scores, and if anything are more autistic-like in their psychological profiles (BaronCohen et al. 1998; Nettle 2006). Both groups have high general intelligence, but it appears to be the artistic types who are attractive for short-term matings and have increased number of partners (Nettle \& Clegg 2006). Hence, it appears that it is not just general intelligence which is a mate-choice indicator, but also the position on the spectrum from hyper-mentalising, psychosis-prone, to hypo-mentalising, autism-prone, with the hypermentalising end of the spectrum the more attractive one.

Two possible explanations spring to mind. One is that mildly hyper-mentalising individuals are better partners to have, because, being people people, they take into account the needs and desires of others. Thus, creative behaviour could simply be signalling one's type in terms of post-reproductive cooperativeness (see Nettle \& Clegg 2007 for some discussion). However, if so, then creative individuals should be especially preferred in the long-term mating context, whereas all the evidence suggests that highly creative individuals tend to follow short-term mating strategy and are preferred in the short-term, genetic-contribution-only context, rather than for long-term investment.

C\&B's article suggests an intriguing alternative: hyper-mentalising, psychosis-prone males are signalling that their genotype is such that their babies will not make excessive demands on their 
Commentary/Crespi \& Badcock: Psychosis and autism as diametrical disorders of the social brain

mothers. C\&B show that psychosis-proneness is associated with smaller, slower growing, less demanding babies, whereas autism-proneness is associated with the opposite. They also argue that the remote associations found in schizotypy and artistic creativity are a direct (and therefore unfakable) consequence of having a brain whose growth pattern is of the psychosisprone - and therefore less costly for the mother - type.

This hypothesis is not implausible. Human reproduction makes extremely high metabolic demands on females. Rates of maternal death in pregnancy, and during or soon after birth, are as high as $2 \%$ per pregnancy in the poorest countries even today (World Health Organisation 2007), and would have been higher under ancestral conditions. The Haigian model of paternal-maternal conflict under multiple paternity predicts that paternal interests will tolerate a greater risk of harm or death to the mother than would be optimal from the point of view of the maternal genome. This tussle is partly carried out at the level of genetic imprints and counter-imprints, but can also be carried out in the arena of mate choice. If the female can find cues that discriminate males on the basis of how strongly their genotype is going to favour the patrilineal interest, she should exploit them. Such cues should be of particular interest in the short-term mating case, where genetic material is all that is being provided. In the long-term case, the additional costs of offspring of autistic-prone individuals could be offset by their propensity to make paternal behavioural investment.

The hypothesis, speculative as it is, could lead to testable predictions. Further investigation is needed into whether the young offspring of highly schizotypal or creative males are indeed smaller or less demanding than the babies of more autisticprone individuals, and also of women's intuitions about the desirability of such men as partners. One prediction might be that women whose own somatic resources are limited (health problems, small size) should be especially keen on schizotypal-creative versus autism-prone males as partners. This would indicate that these traits are being used as mate-choice indicators for the reasons invoked.

It is a great testament to the breadth and importance of C\&B's target article that it allows novel predictions to be generated about something so far removed from their initial concern.

\section{Cortical plasticity: A proposed mechanism by which genomic factors lead to the behavioral and neurological phenotype of autism spectrum and psychotic-spectrum disorders}

doi:10.1017/S0140525X08004378

\author{
Lindsay M. Oberman and Alvaro Pascual-Leone \\ Berenson-Allen Center for Noninvasive Brain Stimulation, Department of \\ Neurology, Beth Israel Deaconess Medical Center and Harvard Medical \\ School, Boston, MA 02215. \\ loberman@bidmc.harvard.edu \\ apleone@bidmc.harvard.edu
}

\begin{abstract}
Crespi \& Badcock $(\mathrm{C} \& \mathrm{~B})$ hypothesize that biases toward expression of paternally or maternally imprinted genes lead to the symptoms of autism spectrum disorders (ASD) and psychotic-spectrum disorders (PSD), respectively. We suggest that such genetic risk factors may act by inducing abnormalities in developmental and learningrelated plasticity. We provide preliminary evidence of abnormal plasticity in ASD and suggest transcranial magnetic stimulation as a useful tool to investigate as well as influence cortical plasticity.
\end{abstract}

The target article suggests that the symptoms of autism spectrum disorders (ASD) and psychotic-spectrum disorders (PSD) are mediated by altered biases toward paternally or maternally expressed genes, respectively. This hypothesis fits well with the existing literature, and provides a potential genotype that acts together with brain development and environmental factors to create the complex phenotypes that define these disorders.

Though understanding the genetic underpinning of these disorders is of critical importance, diagnosis usually occurs around age 2-3 in ASD and during adolescence or later in PSD. Thus, the phenotypic presentation is a result of the complex interaction among genetic risk factors, brain development, and environment, and ultimately the manifestation of brain plasticity. It is worth considering whether the mechanisms of plasticity might be normal, simply acting upon a genetically determined abnormal substrate, or may themselves be pathological, thus contributing to progressively dysfunctional states.

There are several pieces of evidence to support abnormal plasticity in ASD. First, the developmental trajectory of brain size abnormalities suggests a dynamic underlying process. Second, studies have found decreased cortico-cortical and long-range connections and an increase in intracortical and superficial connections. Such patterns of abnormal connectivity might be the result of increased ability to form new connections and/or a decreased ability to prune superfluous ones. Abnormal plasticity is also consistent with the phenotype of PSD, which has also been reported to have abnormal connectivity patterns. Though there are inconsistencies in the findings, one study finds increased corpus callosum size with decreased local connections (Siekmeier \& Hoffman 2002). Additionally, the lower levels of BDNF in schizophrenia (Moises et al. 2002; Palomino et al. 2006; Weickert et al. 2003) may also be related to abnormal plasticity. A recent study suggests that there is a relationship between having a specific polymorphism in the BDNF gene and experience-dependent motor plasticity (Kleim et al. 2006).

Abnormal plasticity provides a plausible, parsimonious, and testable mechanism, consistent with both the main proposal of the target article, as well as the core phenotypes of ASD and PSD. Studies are currently underway to evaluate the role of plasticity in the etiology of ASD using transcranial magnetic stimulation (TMS). TMS paradigms can capture aspects of homosynaptic and heterosynaptic plasticity (see Hallett 2007 for a review). A paradigm known as theta burst stimulation (TBS) is well-suited for investigating homosynaptic plasticity and allows a rapid conditioning of the motor cortex that produces controllable, consistent, and long-lasting effects on motor physiology and behavior. In typical individuals, TBS for 20-90 seconds results in suppression of the motor evoked potential (MEP) for up to 30 minutes. Preliminary data suggest this effect is greater in both degree and duration in individuals with ASD. (See Fig. 1.)

Paired associative stimulation (PAS) is well-suited for investigations of heterosynaptic plasticity and ability for the motor cortex to form long term potentiation (LTP) and long term depression (LTD) in response to sensory input. PAS involves stimulation of the median nerve combined with TMS over the motor cortex at variable interstimulus intervals (ISI). At 25 msec ISI the MEP shows facilitation, while an ISI of $10 \mathrm{msec}$ inhibits the MEP. Both effects are amplified in individuals with ASD. (See Fig. 2.)

Abnormal patterns of plasticity may be leading to the abnormal connectivity in ASD, which in turn may be mediating the deficits in social and communicative skills (as these skills require synchronization of neuronal populations across relatively disparate regions of the cortex) as well as the restricted, repetitive, and stereotyped patterns of behaviors, interests, and activities. Abnormal plasticity may also lead to increased long-range connections in PSD, perhaps contributing to the complex social delusions, enhanced imagination and creativity, and "loose" associations between words and aspects of the environment in this population.

Though the genetic predisposition toward maternally or paternally expressed genes might predispose a child for developing these disorders, the recent increase in incidence can better be 
Commentary/Crespi \& Badcock: Psychosis and autism as diametrical disorders of the social brain

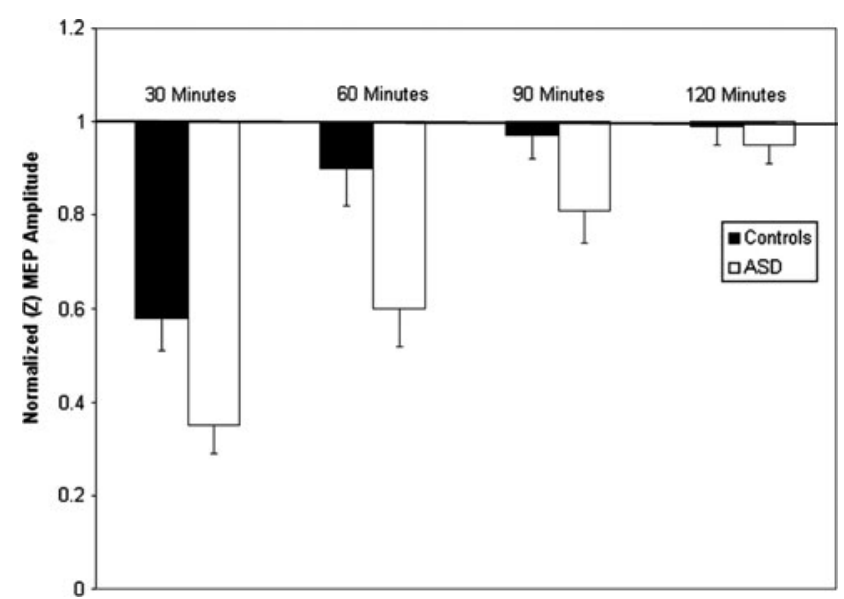

Figure 1 (Oberman \& Pascual-Leone). Bars represent the standardized ( $\mathrm{Z}$ score) MEP amplitude at 30, 60, 90, and 120 minutes following continuous theta burst stimulation (cTBS) for the control and ASD sample. cTBS induces an LTD-like phenomenon that can be quantified as a reduction in the amplitude of the MEP. Error bars represent the standard error of the mean. Note the greater degree and longer duration of suppression in the ASD group.

accounted for by a change in environment rather than an increase in the penetrance of the genetic risk factor. Children growing up in recent years are being exposed to an ever more diverse and stimulating environment packed with stimuli to process from very early on. In a child who is predisposed to increased plasticity, this overly stimulating environment could lead to deleterious consequences. In such a setting, ASD genetic risk factors may lead to a facilitated, insufficiently controlled reshaping of neural connections. The child may develop local hyperconnectivity, a surge in brain size, and an inefficient processing system for incoming sensory stimuli. If, however, this child is placed in a very structured environment and develops a specific interest, this enhanced plasticity may actually lead to savant abilities.

One can also consider the opposite scenario with a child who is genetically at risk for developing PSD with an increased bias

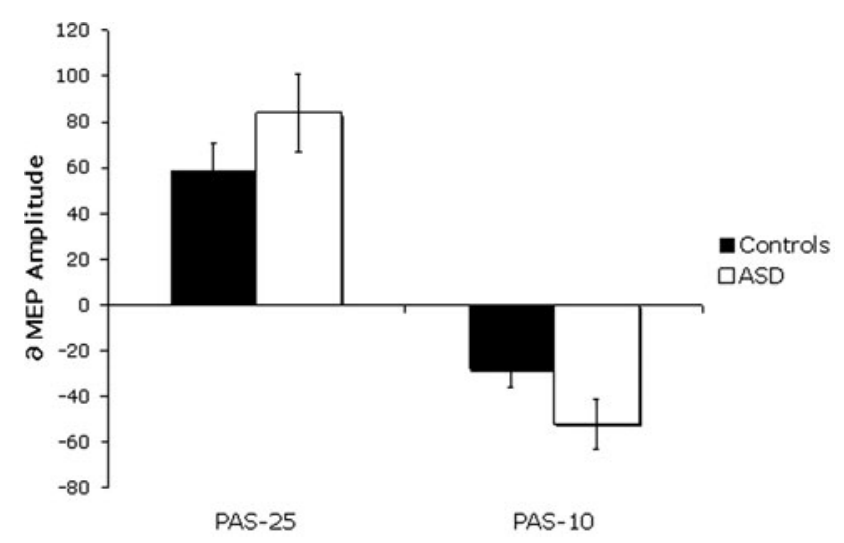

Figure 2 (Oberman \& Pascual-Leone). Bars represent the average percent change in MEP amplitude following paired associative stimulation (PAS) (with an ISI of $25 \mathrm{msec}$ and $10 \mathrm{msec}$ ) for the control and ASD sample. $\mathrm{PAS}_{25}$ results in facilitation, while $\mathrm{PAS}_{10}$ results in suppression of the MEP. Note the greater degree of both facilitation and suppression in the ASD group. Error bars represent the standard error of the mean. toward effects of genes with maternal expression leading to uncontrollable increased global connectivity. Later in life when more associative long-range connections are maturing, abnormal plasticity in this population would lead to the characteristic positive symptoms of PSD.

TMS can be used not only to measure the degree of plasticity in a given system, but also as a valuable tool for clinicians developing therapeutic interventions for these disorders. Specifically, repetitive TMS (rTMS) has been shown to produce long-term (several-month) changes in cortical plasticity and has been effective in other brain diseases, for example, depression.

In conclusion, Crespi \& Badcock $(\mathrm{C} \& \mathrm{~B})$ present an interesting hypothesis for genetic factors that may lead to ASD and PSD. We suggest that abnormal plasticity may be the common underlying mechanism that ultimately leads to many of the core behavioral deficits which define these disorders. The manifestation of the specific pattern of abnormal plasticity may lie in the timing of the hyperplastic response. Specifically, if hyperplasticity is promoted early in life, this may preferentially affect local circuits, resulting in ASD. However, PSD may result from abnormal plasticity during adolescence and young adulthood, leading to promotion of long-range hyperplasticity. Such conceptualization provides an appealing account for the increase in incidence of ASD and PSD in our highly stimulating society. Finally, modulation of plasticity may provide an effective means to treat these disorders.

\section{A complete theory of psychosis and autism as diametric disorders of social brain must consider full range of clinical syndromes}

\author{
doi:10.1017/S0140525X0800438X
}

Katharine N. Thakkar, Natasha Matthews, and Sohee Park

Department of Psychology, Vanderbilt University, Nashville, TN 37240.

katy.thakkar@vanderbilt.edu

natasha.matthews@vanderbilt.edu

sohee.park@vanderbilt.edu

URL: http://www.psy.vanderbilt.edu/faculty/sohee/

Abstract: We argue that autism and psychosis spectrum disorders cannot be conceptualized as polar extremes of mentalizing ability. We raise two main objections: (1) the autistic-psychotic continuum, as conceptualized by the authors, excludes defining features of schizophrenia spectrum: negative symptoms, which correlate more strongly with mentalizing impairments; and (2) little evidence exists for a relationship between mentalizing ability and positive symptoms.

Crespi \& Badcock's (C\&B’s) novel and bold theory places autism spectrum disorders (ASD) and psychotic-spectrum disorders (PSD) on diametrically opposite ends of a spectrum, with underdeveloped social cognition in ASD and hyper-developed social cognition in PSD. The authors represent schizophrenia at the extreme end of PSD and discuss their genetic imprinting hypothesis of hyper-mentalizing in PSD mainly in the context of schizophrenia. However, although the authors acknowledge the multifaceted nature of the disorder, they fail to adequately consider it in the construction of their theory.

One underlying problem with placing schizophrenia and ASD at extreme poles is that the major symptoms of schizophrenia do not cohere with this method of distinction. Schizophrenia is characterized by positive symptoms such as delusions and hallucinations, and negative symptoms that reflect a diminution or loss of normal functions such as alogia, anergia, and anhedonia. Positive symptoms fluctuate over time and can be ameliorated by antipsychotic medication, but negative symptoms are treatment-resistant and enduring. C\&B's theory focuses on schizophrenia to represent 
Commentary/Crespi \& Badcock: Psychosis and autism as diametrical disorders of the social brain

the psychotic spectrum, yet the more trait-like negative symptoms are largely ignored.

The process of hyper-mentalizing, which results in increased suspiciousness, is best related to one component of positive symptoms, namely, paranoia and persecutory delusions. Although it is a theoretically reasonable argument that hyper-mentalizing is related to paranoid delusions, it is difficult to make the same argument for the negative symptoms. Thus the borders of C\&B's autistic-psychotic continuum must be redrawn.

It is possible to constrain C\&B's theory to address the positive rather than negative symptoms, but empirical data pose further complications. Here we focus on one aspect of social cognition, theory of mind (ToM), which is the ability to attribute mental states to oneself and others, and we consider the nature of ToM deficits in schizophrenia and the relationship between these deficits and clinical symptoms. An abundance of empirical evidence points to mentalizing deficits in both PSD (i.e., schizophrenia, schizotypy) and ASD. C\&B propose that the underlying deficit determining impaired social cognition in autism is an inability to theorize about the mental states of others (hypo-mentalizing). Indeed, evidence for ToM deficits in autism is robust (BaronCohen 1995). In the case of schizophrenia, there is also good evidence for the presence of ToM deficit (for review, see Brüne 2005), but very little evidence exists for the hypothesis that schizophrenia involves hyper-mentalizing about the mental states of others. Moreover, ToM deficits in schizophrenia may stem from executive or planning deficits (Hardy-Baylé 1994) or an inability to integrate contextual information (Hardy-Baylé et al. 2003). Thus, both ASD and PSD involve impaired social cognition, but the reasons for these deficits may not lie on the same continuum of mentalizing ability.

With respect to the positive symptoms, persecutory delusions may arise when individuals mislabel actions of others (Frith 1992), and therefore one may expect a correlation between persecutory delusions and ToM deficits. However, evidence for this relationship is inconclusive at best, in contrast to the more consistent evidence of a relationship between negative symptoms and ToM deficits (Freeman 2007). C\&B point out that psychotic symptoms are not just present in schizophrenia, but also present in other conditions, including depressive psychosis, bipolar disorder, posttraumatic stress disorder (PTSD), paranoid personality disorder, dementia, and epilepsy. Persecutory delusions are one of the most common features of psychosis, occurring in almost $50 \%$ of cases (Sartorius et al. 1986). If paranoid ideation and persecutory delusions are the components of psychosis most directly linked to hypermentalizing, then there should be an association between these experiences and ToM deficits. However, Walston et al. (2000) found no evidence for ToM deficits in participants with pure persecutory delusions.

Furthermore, $\mathrm{C} \& \mathrm{~B}$ argue that non-pathological points on the psychosis spectrum are most useful in evaluating their hypothesis. However, even in healthy schizotypal individuals, those who score high on psychometric measures of schizotypal personality, ToM deficits were related to the negative syndrome and not with the positive syndrome (Langdon \& Coltheart 1999). With respect to the non-pathological individuals on the spectrum, there is an additional complication. Machiavellian intelligence, that is, social intelligence used as a means of social manipulation in order to attain goals, can improve social cognitive functioning when applied appropriately in context. Therefore, controlled and intentional hyper-mentalizing, even if extreme, should correlate with enhanced ToM and effective social behavior. In other words, is there room for Sherlock Holmes in C\&B's spectrum?

Moreover, whether mentalizing impairments in PSD are state or trait features is still inconclusive. Although, generally, the data lend more support to a trait hypothesis, it is impossible to confirm without longitudinal studies (Harrington et al. 2005b). This is perhaps further evidence for the importance of negative symptoms for ToM deficits in schizophrenia, given that negative symptoms are a more stable feature of the illness than positive symptoms, which vary over the course of the illness and with treatment.

To conclude, we believe there is not a strong enough empirical platform to assert impaired mentalizing, and especially hypermentalizing, as a phenotypic marker of PSD, which C\&B's subsequent genetic imprinting theory requires. Evidence regarding mentalizing deficits being a state or trait marker of PSD is mixed, and it is equally unclear whether impaired task performance in PSD is caused by deficits in mentalizing or other cognitive problems. We argue that autism and schizophrenia cannot easily be conceptualized as polar opposites on a spectrum or continuum of mentalizing ability without changing the nature of this spectrum. However, with some modifications, C\&B's theory lends itself readily to empirical testing and hence provides a valuable framework for future research.

\section{Reunifying autism and early-onset schizophrenia in terms of social communication disorders}

\author{
doi:10.1017/S0140525X08004391
}

\section{Sylvie Tordjman}

Department of Child and Adolescent Psychiatry, University of Rennes 1 and Laboratoire de Psychologie de la Perception, CNRS FRE 2929, University of Paris 5, France.

s.tordjman@ch-guillaumeregnier.fr

\begin{abstract}
Autism and early-onset schizophrenia share common dimensions of social communication deficits. The possible role of common genetic factors has to be seriously considered, such as the serotonin transporter gene that influences the severity of social communication impairments (negative symptoms) and hallucinations (positive symptoms). Autism and the negative syndrome of schizophrenia might be at one extreme of a continuum, and paranoid schizophrenia (positive symptoms) at the other extreme.
\end{abstract}

Autism and schizophrenia (more precisely, the negative syndrome of schizophrenia as observed in early-onset schizophrenia), far from being diametrical disorders of the social brain as postulated by Crespi \& Badcock (C\&B), share common psychopathological dimensions in terms of social communication deficits. This does not contradict C\&B's hypothesis that autism and schizophrenia (but the latter in its paranoid form) represent two extremes on a cognitive spectrum.

Historical diagnostic overlaps between autism and schizophrenia have existed since the appearance of autism in the nomenclature of psychiatric disorders. Originally autistic disorder and schizophrenia were intimately linked. Indeed, the term autism is derived from the Greek autos, which means self and was introduced for the first time by the Swiss psychiatrist Eugen Bleuler in 1911 to describe social withdrawal in adults with schizophrenia. In 1943, the American psychiatrist Leo Kanner borrowed from Bleuler the term autism to define a syndrome observed in 11 children - which, at that time, was a part of the diagnostic category "Childhood Schizophrenia." Since the early 1970s, autism and schizophrenia have belonged to two different diagnostic categories. However, they still share some common features: They are both considered as developmental psychiatric disorders involving psychotic symptoms with impairments in the same main behavioral domains (especially in communication and social interactions). Communication impairments are reported in autistic disorder as well as in early-onset schizophrenia (Alaghband-Rad et al. 1995; Asarnow et al. 1994; Baum et al. 1995; Cantor et al. 1982). Thus, impairments in verbal communication (delay in the development of spoken language, poor or disorganized speech) and nonverbal communication (reduced facial expression or body language; poor eye contact; and abnormal emotional expression 
Commentary/Crespi \& Badcock: Psychosis and autism as diametrical disorders of the social brain

such as flat, bizarre, or inappropriate affects) are found in individuals with early-onset schizophrenia or autistic disorder. In addition, social development is impaired in both schizophrenia and autistic disorder. The degradation of social skills is a hallmark of schizophrenia (Mueser et al. 1991) and the distinguishing feature of subgroups within schizophrenia (Carpenter et al. 1988). Deterioration of social skills is associated with the chronic phase of schizophrenia and its deficit form (Carpenter et al. 1988) or negative syndrome. Social isolation observed in schizophrenia, in particular in the childhood of individuals with earlyonset schizophrenia, is similar to the autistic withdrawal described by Kanner (1943).

Tantam (1988) even argued that individuals with autistic disorder display symptoms that can also be considered as schizoid personality traits. More recently, Konstantareas and Hewitt (2001) reported that none of 14 men with paranoid schizophrenia met criteria for autism, whereas 7 out of 14 males with autism met criteria for schizophrenia on the Structured Clinical Interview (SCID). More precisely, symptom overlap concerned negative symptoms of schizophrenia, such as affective flattening, alogia, avolition, apathy, anhedonia, and poor communication. This symptom overlap can be observed in particular between high functioning autistic disorder or Asperger's syndrome and early-onset schizophrenia, which tends to be characterized by negative symptoms with social interaction impairments (Bailer et al. 1996) and a chronic course (Krauss et al. 2000). Furthermore, retrospective studies conducted on patients with schizophrenia or longitudinal studies of children with autistic disorder reported frequent associations between these two disorders (antecedents of autistic disorder in the childhood of the schizophrenic group and appearance of schizophrenia in the autistic group) (Alaghband-Rad et al. 1995; Bender \& Faetra 1972; Jansen et al. 2000; Petty et al. 1984; Van Engeland \& Van Der Gaag 1994).

From a biological point of view, abnormal stress responses (in particular responses of the hypothalomo-pituitary-adrenal axis to psychosocial stress) are reported in schizophrenia (Jansen 1998; Jansen et al. 2000) as well as in autistic disorder (for a review, see Tordjman et al. 1997). This common biological feature is associated in both disorders with major anguish focused often on body image, difficulties to adapt to novel situations, and abnormal behavioral responses to environmental stimuli and stressful situations (Jacobson \& Ackerman 1990; Tordjman et al. 1997; Van Den Bosch et al. 1992; Wiedl 1992). Interestingly, some studies suggest that the apparent pain insensitivity observed in both autism and schizophrenia is more related to a different mode of pain expression due to social communication impairments and body image problems, than to a real endogenous analgesia (Guieu et al. 1994; Tordjman et al. 1999; Watson et al. 1979).

Finally, genetic factors possibly involved in both early-onset schizophrenia and autistic disorder have been discussed by Yan et al. (2000). The serotonin transporter gene $(H T T)$ is of particular interest given the reported association of a HTT promoter polymorphism with anxiety-related traits in the general population (Lesch et al. 1996). Furthermore, in a genetic study conducted on families of 71 children with autism, we showed that the HTT promoter polymorphism modifies the behavioral phenotypic expression of autism: the data consistently pointed to the short $(s)$ allele being associated with the more severe impairments in the communication and social interaction domains (Tordjman et al. 2001). These data were confirmed by another study (Brune et al. 2006). Of special relevance are reports that the HTT promoter polymorphism also influences the severity of hallucinations in schizophrenia: Intensity of hallucinations was positively associated with the long ( $l$ ) allele (Malhotra et al. 1998) and negatively associated with the $s$ allele (Golimbet et al. 2003). It suggests that the HTT promoter polymorphism influences the phenotypic expression of positive symptoms (hallucinations) and negative symptoms (social withdrawal and communication deficits). In this regard, our results fit within C\&B's hypothesis: Autism and the deficit form of schizophrenia (observed in particular in early-onset schizophrenia) would be at one extreme (negative symptoms), and paranoid schizophrenia at the other extreme (positive symptoms) of this continuum. It opens the path to future common research on the genetics of autism and schizophrenia, and underlines the importance of using a methodology in which genetic transmission is studied in concert with a detailed examination of clinical phenotype.

In conclusion, I suggest that future research on the genetics of autism and schizophrenia should develop multi-trait models (Tordjman et al. 2007) and a multidimensional approach, such as studying social and communication impairments, instead of focusing on diagnostic categories.

\section{Psychiatric disorders and the social brain: Distinguishing mentalizing and empathizing}

doi:10.1017/S0140525X08004408

\author{
Alfonso Troisi \\ Department of Neuroscience, University of Rome, Tor Vergata, 00161 Rome, \\ Italy. \\ alfonso.troisi@uniroma2.it
}

Abstract: Social cognition is a broad term, incorporating all aspects of social functioning from perceiving emotional stimuli to attributional style and theory of mind. Not distinguishing between these different capacities may confound the interpretation of the data deriving from studies of the relationship between psychiatric disorders and the social brain. The distinction between cognitive and affective components of social cognition is clearly exemplified by the abnormalities observed in psychopathy and Williams syndrome.

Traditionally, assessment and diagnosis of psychiatric disorders have focused on affective, cognitive, and somatic symptoms. However, interpersonal problems and abnormal social behaviors are often the first indication than an individual is experiencing mental health difficulties. Recent advances in the field of social neuroscience have been instrumental in showing that abnormalities in social cognition are an important aspect of the clinical profile of many psychiatric disorders, and that some interpersonal problems associated with psychopathology are caused by dysfunctions in the social brain (Brüne et al. 2003). In line with this new approach to defining the clinical phenotypes of psychiatric disorders, Crespi \& Badcock (C\&B) focus on social brain functioning in autism and psychosis. After reviewing an impressive amount of clinical, behavioral, and neurological data, C\&B conclude that these two conditions represent opposites along a spectrum from underdeveloped to hyperdeveloped social cognition, with normality at the center, and hypothesize that the development of these disorders is mediated by genomic imprinting.

Whereas it is undisputed that patients with autism display deficits in a wide range of social cognition tasks, the idea that psychosis (and schizophrenia in particular) is associated with a hyperdeveloped social brain is likely to leave many clinicians perplexed. In fact, it seems to go against the prevailing consensus that patients with schizophrenia show defective (rather than enhanced) abilities in the domain of social cognition (Burns 2006b). However, before considering C\&B's view of schizophrenia as heretical, we should pay attention to the methodological problems that complicate the study of social brain dysfunctions in psychiatric disorders.

First, psychiatric diagnoses in general, and the diagnosis of schizophrenia in particular, lack validity. Patients with the same diagnosis may have different diseases, and these different diseases (or subsyndromes) may involve different abnormalities of the social brain. For example, in the schizophrenia population, social cognition abilities seem to vary with the presence of 
Commentary/Crespi \& Badcock: Psychosis and autism as diametrical disorders of the social brain

violent behavior (Abu-Akel \& Abushua'leh 2004), paranoid features (Langdon et al. 2002), and negative symptoms (Corcoran \& Frith 1996). Second, tasks designed for studying social cognition in psychiatric patients tend to have dubious ecological validity. For example, McCabe et al. (2004) showed that individuals with schizophrenia demonstrate intact theory-of-mind abilities when tested in real life during conversational interactions. Third, the term "social cognition" is a broad concept, incorporating all aspects of social functioning from perceiving social emotional stimuli to attributional style and theory of mind. Not distinguishing between the different capacities that allow an individual to navigate the social environment may confound the interpretation of the data deriving from studies of the relationship between psychiatric disorders and the social brain. Here, I will focus in further detail on this last methodological problem.

A promising approach to dissecting the various components of social cognition is to distinguish the capacity to represent other people's intentions and beliefs (referred to as theory of mind or mentalizing) and the capacity to share the feelings of others (referred to as empathy) (Singer 2006). Although C\&B do not pay much attention to such a distinction in their target article, there is preliminary evidence that the capacities to mentalize and to empathize are differentially impaired in schizophrenia. Discussing these data, Shamay-Tsoory et al. (2007) have argued that the conflicting evidence in the literature concerning schizophrenic patients' ability to perform on theory-of-mind tasks is due to the dissociation between cognitive and affective components of social cognition. According to these authors, such dissociation would also explain the puzzling finding that the schizophrenic population includes both individuals who "undermentalize" and individuals who "overmentalize" (C. D. Frith 2004). However, probably schizophrenia is not the best model to illustrate the necessity to distinguish between cognitive and affective components of social cognition. In this regard, psychopathy and Williams syndrome are much better.

Psychopathy is a pervasive pattern of disregard for the rights of others associated with distinctive emotional and behavioral features. Individuals with this personality disorder are frequently deceitful and manipulative in order to gain personal profit. Even though they may display a glib, superficial charm, these people tend to be callous, cynical, and contemptuous of the feelings, rights, and suffering of others. The emotional deficits associated with psychopathy interfere with the development of moral reasoning and put the individual at risk for developing high levels of antisocial behavior. In other words, an absence of empathy is what characterizes psychopaths who hurt others without feeling guilt or remorse (Blair 2003). Interestingly, the empathic deficit is associated with no other deficit of social cognition. Persons with this disorder are capable of accurately assessing the costs and benefits of short-term social interactions, accurately reading others' behavior rules, utilizing self-monitoring information to alter their strategies, and successfully disguising their intentions (Troisi 2005). Confirming this, experimental studies have found no indications of impairment with theory of mind among individuals with a diagnosis of psychopathy (Richell et al. 2003).

Williams syndrome (WS) is a rare neurodevelopmental disorder caused by hemizygous microdeletion of about 28 genes on chromosome 7 q11.23 and characterized by many cardiovascular, endocrine, gastrointestinal, and orthopedic problems. WS is associated with mild to moderate mental retardation and a unique combination of social-emotional and social-cognitive abnormalities. A striking feature of children with WS is their high sociability and empathy for others. Increased interest in social interaction is evident from infancy onwards, and fascination with faces, positive interpersonal bias, and overfriendliness extend into adulthood. Intriguingly, this remarkable hypersociability is coupled with a deficit in the ability to interpret people's behavior in terms of their mental states. Therefore, despite their increased empathy and social gregariousness, individuals with

WS encounter problems in everyday interactions because of their inability to detect and understand others' beliefs and intentions (Meyer-Lindenberg et al. 2006). In short, the social brain dysfunctions observed in psychopathy and WS appear to be distinct and selective. Individuals with psychopathy show a profound empathic dysfunction but no indications of impairment with theory of mind. In contrast, individuals with WS show abnormally high levels of empathy combined with a defective theory of mind.

The distinction between cognitive and affective components of social cognition exemplified by psychopathy and WS may apply to other psychiatric disorders. Future studies in different diagnostic groups will have to clarify when impaired abilities of mentalizing (understanding the propositional attitudes of others) and empathizing (sharing the affects of others) play unique roles and when they play common roles in causing social disability.

The relationship between psychiatric disorders and the social brain is likely to be multiformed. In some psychiatric disorders, abnormalities in social cognition may be a vulnerability factor that gets transformed into full-blown psychopathology in the face of significant stressors. In other disorders, the experience of psychiatric symptoms may produce temporary abnormalities in social cognition in individuals with effective and appropriate capacities for navigating the social environment. Progress in this area of research requires not only refining methods for assessing the various aspects of social cognition, but also developing theoretical models for generating new explicative hypotheses. In this regard, C\&B's target article is an important contribution.

\section{Psychosis and autism as two developmental windows on a disordered social brain}

\section{doi:10.1017/S0140525X0800441X}

\section{Sophie van Rijn, ${ }^{a}$ Hanna Swaab, ${ }^{a}$ and André Aleman ${ }^{b}$ \\ ${ }^{a}$ Department of Clinical Child and Adolescent Studies, Centre for the Study of Developmental Disorders, Leiden University, 2333 AK Leiden, The Netherlands; ${ }^{\mathrm{b}} \mathrm{BCN}$ Neuroimaging Center, University of Gröningen, 9713 AW Gröningen, The Netherlands. srijn@fsw.leidenuniv.nl hswaab@fsw.leidenuniv.nl a.aleman@med.umcg.nl}

Abstract: With regard to social-cognitive deficits in autism and psychosis, Crespi \& Badcock's (C\&B's) theory does not incorporate the developmental context of the disorders. We propose that there is significant overlap in social-cognitive impairments, but that the exact manifestation of social-cognitive deficits is highly dependent on the dynamics of cognitive development and hence different in autism as compared to psychosis.

We begin by stating that the theoretical framework of Crespi \& Badcock $(\mathrm{C} \& \mathrm{~B})$ is an important contribution to the unraveling of the nature of disorders of the social brain. However, although it is not our intention to question the consensus that autism and schizophrenia are two different psychiatric disorders, we argue that the described differences in brain functioning and cognition between autism and psychosis should be considered within a developmental context. The comparison of social-cognitive dysfunctions in autism and psychosis as $\mathrm{C} \& \mathrm{~B}$ have presented, implies in most cases a comparison of two different developmental windows. Autism is by definition a childhood-onset disorder, whereas psychosis is diagnosed primarily in (early) adulthood. This may have important implications, as findings from developmental neuropsychology suggest that the same cognitive dysfunctions may present differently at different ages.

First, depending on the maturation of the brain, specific cognitive functions become "on-line" as development progresses. For example, maturation of the frontal cortex continues into 
Commentary/Crespi \& Badcock: Psychosis and autism as diametrical disorders of the social brain

early adulthood, and executive functions will therefore be quantitatively and qualitatively different in early adulthood as compared to childhood. Hence, certain cognitive impairments will become more salient as development progresses. Considering this "growing into deficit," a concept proposed by Rourke et al. (1983), the profile of cognitive impairments may change over time in individuals with a neurodevelopmental disorder. Hence, the pattern of behavioral difficulties may also change over time in some individuals possibly resulting in a shift in psychopathology. Recent reports suggest that shifts from the autism spectrum to the psychosis spectrum are mainly seen when considering the broad autism spectrum, that is, children who have severe social impairments but who may not meet full criteria for the core autism phenotype. Mouridsen et al. (2008) have followed up 89 children with atypical autism, of which $30.4 \%$ developed a psychotic disorder in adulthood, whereas only $1.9 \%$ of the 258 non-clinical controls did so. Second, Karmiloff-Smith (1997) has stressed the importance of considering the interactive nature of cognitive impairments associated with neurodevelopmental disorders. Thus, the neurodevelopmental origins of two distinct phenotypical outcomes, such as autism and psychosis, may show similarities. However, the exact phenotypical outcome is shaped by interactions with other neurodevelopmental and environmental factors during development.

Although autism and schizophrenia can quite reliably be separated as two distinct syndromes by DSM-IV or IDC-10 criteria, at the level of individual symptoms some of the behavioral characteristics are observed in both disorders. This is not new, as Bleuler (1911) already delineated "autism" as a cardinal feature of schizophrenia. C\&B attribute this overlap to superficial behavioral similarities and argue that autistic features may also be expressed in some negative schizophrenia symptoms. However, there is also evidence for increased levels of paranoid traits in individuals with Asperger's syndrome (Blackshaw et al. 2001; Craig et al. 2004), suggesting that behavioral overlap is not limited to negative schizophrenia symptoms and extends to psychotic traits. Taking this together with the dynamics of cognitive development, we argue that although social-cognitive impairments may seem different in (adults with) psychosis as compared to (children with) autism, this does not necessarily point to two different or opposite social brain dysfunctions.

Indeed, reports of similarities in social-cognitive performance in both disorders support this suggestion. Besides mentalizing deficiencies in autism, a recent meta-analysis has pointed to deficits in theory of mind (ToM) in different subtypes of schizophrenia patients (Sprong et al. 2007). Not only the schizophrenia patients characterized by disorganized or negative symptoms, but also those with predominantly persecutory delusions scored significantly lower in each study, on average 1.2 standard deviations lower than non-clinical controls, in various tasks measuring mentalizing and ToM capacities. Moreover, in a study by Harrington and colleagues (Harrington et al. 2005a), the severity of persecutory delusions in schizophrenia patients was related $(r=-.48)$ to more severely impaired mentalizing abilities. Also, patients with paranoid delusions could not be discriminated from individuals with Asperger syndrome on several tasks measuring ToM skills (Craig et al. 2004), as both groups performed comparably worse as compared to non-clinical controls. Not only in clinical populations, but also in the general population, "positive" schizotypal traits (analogous to psychotic clinical symptoms) seem to predict poorer mentalizing performance (Pickup 2006). One possible explanation for the relation between psychosis or paranoia and ToM impairments has been put forward by Bentall and Kinderman (1998). They propose that the following two factors contribute to persecutory delusions: (1) an external attributional bias, and (2) an impairment in incorporating contextual information needed for making attributions. An impaired ability to understand what other persons are thinking and feeling (ToM) represents one type of deficit in taking into account important contextual information.
Interestingly, similarities between the autism and psychotic spectrum have also been observed with regard to the neurobiological underpinnings of impaired theory of mind. When asking individuals to judge faces with regard to trustworthiness, that is, attribute intentions to others, functional magnetic resonance imaging (fMRI) shows very similar patterns of brain activation in individuals with an autism-spectrum disorder and paranoid schizophrenia patients (Pinkham et al. 2008).

Finally, our studies on Klinefelter syndrome also suggest an overlap in social-cognitive dysfunctions associated with autistic and psychotic behavior. Men with Klinefelter syndrome display various social-cognitive deficits and have increased levels of both autistic and psychotic traits (van Rijn et al. 2006a; 2006b; 2007; in press).

In sum, we feel that C\&B's comparison of the cognitive mechanisms characteristic of the autism spectrum and psychotic spectrum should take into account the dynamics of cognitive development from childhood into adolescence and adulthood. Also, the observations of similarities in social-cognitive dysfunctions in the autism and schizophrenia spectrum should not be ignored. We hope to have provided convincing evidence showing that mentalizing abilities, which are among the core domains of cognitive functions that dissociate autism from psychosis according to $\mathrm{C} \& \mathrm{~B}$, are hypodeveloped in both the autism and psychotic spectrum. As also suggested by Pinkham et al. (2008), social brain dysfunctions in the autism and psychotic spectrum may be deficit-specific, rather than disorder-specific.

\section{Evolutionary perspectives on psychoses and autism: Does genomic imprinting contribute to phenomenological antithesis?}

\author{
doi:10.1017/S0140525X08004421
}

\section{Ganesan Venkatasubramanian}

Department of Psychiatry, National Institute of Mental Health and

Neurosciences, Bangalore, Karnataka, 560029, India.

venkat.nimhans@gmail.com

http://venkat.nimhans.googlepages.com/home

\begin{abstract}
Crespi \& Badcock $(\mathrm{C} \& \mathrm{~B})$ have presented a novel view that the influence of genomic imprinting causes diametrically opposite disorders: namely, psychoses and autism. I propose an extended hypothesis that while genomic imprinting is likely to have an influence on the pathogenesis of psychoses and autism, it might contribute to phenomenological antithesis between as well as within these disorders.
\end{abstract}

Crespi \& Badcock $(\mathrm{C} \& \mathrm{~B})$ have elegantly summarized the evidence for psychoses and autism to be considered as "diametrical disorders of the social brain." Importantly, their work blends the concept of differential evolutionary selection pressures on male and female brains (Lindenfors et al. 2007) with contemporary neurobiological observations. Although the authors have described autism and psychoses as "diametrically opposite phenotypes" (target article, Abstract), evidence from other studies (reviewed in this commentary) suggests that the relationship between them might be more complex.

This intriguing complexity is illustrated by the striking comorbidity of "seemingly incompatible" syndromes (e.g., $34.8 \%$ of adults with atypical autism had schizophrenia spectrum disorders; Mouridsen et al. 2008). Also, in the recently postulated "network disease hypothesis," it was estimated that 20-60\% of autism-predisposing genetic polymorphisms also predispose the bearer to bipolar disorder, and $20-75 \%$ of these polymorphisms also predispose the bearer to schizophrenia (Rzhetsky et al. 2007). Moreover, DISC1 gene, which was reported to co-segregate with schizophrenia (Mackie et al. 2007), is also associated with autism and Asperger's syndrome (Kilpinen et al. 2007). 
Commentary/Crespi \& Badcock: Psychosis and autism as diametrical disorders of the social brain

In addition to these shared genetic diatheses, evidence for potentially similar pathogenesis for both autism and schizophrenia does exist (although the studies summarized in Table 1 of the target article suggest contrasting pathogenetic mechanisms). Both autism and schizophrenia are associated with (1) deficient insulin-like growth factor-1 (Riikonen et al. 2006; Venkatasubramanian et al. 2007), (2) masculinized digit ratio (Manning et al. 2001; Procopio et al. 2006), (3) smaller corpus callosum (see Waiter et al. [2005] in relation to autism; see Venkatasubramanian et al. [2002] and Woodruff et al. [1995] in relation to schizophrenia), and (4) increasing paternal age (Reichenberg et al. 2006; Sipos et al. 2004).

These shared genetic and pathogenetic abnormalities, as well as clinical comorbidity between psychoses and autism, suggest an extended hypothesis that, although genomic imprinting is likely to have an influence on the pathogenesis of psychoses and autism, it might contribute to phenomenological antithesis (opposite symptomatic manifestations) between as well as within these disorders. This hypothesis derives indirect support from the recently emerging view that no unitary cause (genetic or cognitive) can explain the diverse symptoms of autism (Happé et al. 2006). Extending the thesis by Happé et al. (2006), I propose that at least three evolutionary significant dimensions - namely, systemizing ability, schizotypy, and affect regulation (with each dimension being independent and possibly having multiple components) - need careful consideration with relevance to the impact of genomic imprinting on these disorders. Interestingly, the prototypical disorders related to these three dimensions (systemizing ability - autism; schizotypy - schizophrenia; affect regulation - bipolar disorder) are all influenced by genomic imprinting (Luedi et al. 2007).

Recently, Luedi et al. (2007) have listed genes that have been proved or predicted with high confidence to be imprinted in loci linked to these three disorders. The ratio of paternal to maternal (P:M) effects (parent-of-origin as well as paternal/maternal expression) of the proposed imprinted genes in these three disorders are as follows: autism $(\mathrm{P}: \mathrm{M}=5: 4)$, schizophrenia $(\mathrm{P}: \mathrm{M}=3: 2)$, and bipolar disorder $(\mathrm{P}: \mathrm{M}=3: 6)$ (Luedi et al. 2007; supplemental research data online). These P:M ratios suggest that the influence of genomic imprinting on these disorders may not be exclusively paternal or maternal. The P:M profiles of autism and schizophrenia suggest more of paternal than maternal effects, whereas the P:M profile of bipolar disorder suggests the reverse.

Based on this, one might speculate that autism and bipolar disorder are "phenomenologically antithetical" disorders (at either end of spectrum) with schizophrenia being "in the middle." Interestingly, the multidimensional model of schizotypy (Kwapil et al., in press) suggests that "positive schizotypy" is related to psychoticlike experiences and mood disorders (tending more towards bipolarity), whereas "negative schizotypy" is related to negative and schizoid symptoms (tending more towards autism). Similar descriptions for affective instability (Lara et al. 2006), as well as systemizing ability (Baron-Cohen 2006), can explain the spectrum of symptoms within a specific dimension, as well as the paradoxical coexistence of apparently "polar" or phenomenologically antithetical conditions.

Genetic studies of these three hypothesized dimensions might offer valuable insights on the pathogenesis of these neuropsychiatric disorders. Because autism, schizophrenia, and bipolar disorder are affected by genomic imprinting (Luedi et al. 2007), it is plausible that the three hypothesized dimensions that are adversely affected in these disorders might also be influenced by imprinting. Indeed, the schizotypal dimension in schizophrenia and bipolar disorder has been reported to be associated with homozygous high activity catechol-O-methyltransferase (COMT). Moreover, hypomethylation of membrane-bound COMT promoter gene has been reported to be a potential molecular basis for the shared symptoms of schizophrenia and bipolar disorder (Abdolmaleky et al. 2006). Significantly, methylation-mediated processes constitute one of the important mechanisms for genomic imprinting (Reik \& Walter 2001). This suggests that schizotypy might be influenced by imprinting.

Imprinting is postulated to offer evolutionary adaptations (Wilkins \& Haig 2003). Interestingly, these hypothesized dimensions are evolutionarily significant because a balanced mix of these dimensions might offer survival advantages (James 2003; Nettle \& Clegg 2006), whereas an imbalance might lead to psychiatric disorders. An imbalanced imprinting polarized to one of these three dimensions might result in one disorder (e.g., extreme schizotypy might result in schizophrenia), whereas an imbalanced imprinting adversely influencing more than one dimension might result in comorbidity of phenomenologically antithetical conditions. Also, the imprinting mechanisms influencing these hypothetical dimensions might be more intricate because of additional factors like "leaky imprinting" (which can result in "gain-of-function" as well as disorder; Delong 2007), environmental influences (Jirtle \& Skinner 2007), and interaction with sex-related biological differences.

To summarize, Dobzhansky's statement that, "Nothing in biology makes sense except in the light of evolution" (Dobzhansky 1973), emphasizes the need for evolutionarily informed approaches to understand diseases and disorders. However, contemporary medicine focuses predominantly on "proximaletiology" whereas the "distal-etiology" based evolutionary approach has mostly been neglected (Nesse et al. 2006). C\&B's target article is an example for promising applications of evolutionary approaches in understanding complex human diseases. Although the studies summarized by $\mathrm{C} \& \mathrm{~B}$ strongly emphasize the influence of genomic imprinting in psychoses and autism, additional literature reviewed in this commentary illustrates the complexity of this influence; and I hypothesize that genomic imprinting contributes to phenomenological antithesis between as well as within these disorders.

\section{Towards a computational neuroscience of autism-psychosis spectrum disorders}

\author{
doi:10.1017/S0140525X08004433
}

\section{Tony Vladusich}

Department of Cognitive and Neural Systems, and Center of Excellence for Learning in Education, Science and Technology, Boston University, Boston, MA 02215.

thevlad@bu.edu

Abstract: Crespi \& Badcock (C\&B) hypothesize that psychosis and autism represent opposite poles of human social cognition. I briefly outline how computational models of cognitive brain function may be used as a resource to further develop and experimentally test hypotheses concerning "autism-psychosis spectrum disorders."

Computational neuroscience seeks to understand brain function through a combination of mathematical and experimental techniques. The computational neuroscience of mental disorders - a relatively new field of research - may provide an avenue to develop and test the hypothesis of Crespi \& Badcock $(\mathrm{C} \& \mathrm{~B})$ that autism and psychosis represent opposite poles of human social cognition. Modifications to existing computational models of healthy brain function, in particular, may help to understand how imbalances in neurotransmitter systems give rise to the behavioral symptoms associated with autism and psychosis.

I will discuss how a computational model of category learning and recognition has begun to inform research on both autism and psychosis (Grossberg 2000a; Grossberg \& Seidman 2006). This model hypothesizes that humans recognize objects by matching top-down expectations (subliminal primes or hypotheses) based on learned categories - against bottom-up representations 
Commentary/Crespi \& Badcock: Psychosis and autism as diametrical disorders of the social brain

of sensory features. According to the model, when there is a sufficient match between top-down categories and bottom-up features, we recognize the observed object as belonging to the selected category. Modifications to the learned associations between features and category can take place at this juncture, thereby refining the category expectation. When there is a sufficiently large mismatch between the categories and features, the brain quickly and unconsciously searches through existing category memories for a better-matching category. Should this search fail, the brain then learns a new category, associating the existing cluster of sensory features with the active category. A model parameter called vigilance determines the amount of similarity required to define a match between categories and feature clusters. If vigilance is high, a high degree of similarity between categories and features is required for recognition to occur, whereas the converse is true if vigilance is low.

It has been proposed that vigilance varies continuously in a population of individuals, and that autistic individuals may have an abnormally high vigilance level (Grossberg \& Seidman 2006). This abnormally high vigilance level ensures that autistic individuals are inordinately influenced by bottom-up features. High vigilance leads to the formation of highly specific (concrete) categories and prevents the learning of general (abstract) categories. In terms of recognition performance, high vigilance means that autistic individuals require an above-average degree of similarity between categories and features to recognize an object (Klinger \& Dawson 2001; cf. Molesworth et al. 2005). It also implies that such individuals often fail to recognize objects, such as faces, due to trivial variations in sensory features, such as changes in hair-style. On the flip side, high vigilance ensures that autistic individuals very seldom mistakenly recognize an object as belonging to a category to which it does not belong: High vigilance will lead to the formation of new categories for almost any cluster of features. Such abnormalities in category learning may secondarily affect related brain regions involved in conditioning, motivation, cognition, and motor control (Grossberg \& Seidman 2006). Thus, according to this modeling framework, many autistic symptoms may arise from abnormalities in brain systems governing basic learning, attention, expectation, and recognition.

The top-down pathways involved in category learning and recognition usually act as subliminal priming signals. This means that top-down signals do not normally activate neurons encoding sensory features, preventing us from experiencing hallucinations during daily life. It has been proposed (Grossberg 2000a), however, that humans are able to volitionally (willfully) modulate the influence of top-down signals, allowing these signals to become suprathreshold. Such suprathreshold top-down activation in the absence of actual sensory features is hypothesized to occur during normal imagery, such as inner speech. During psychotic episodes, according to this theory (Grossberg 2000a), top-down signals become suprathreshold due to the loss of volitional modulatory control, leading to hallucinations - a hypothesis for which there exists some empirical support (Aleman et al. 2003). The same modeling framework which helps to explain aspects of autism, is therefore able to account for features of psychosis.

Can the autistic and psychotic features in the above computational framework be considered opposite poles of a spectrum? Probably not, strictly speaking. The high vigilance associated with hyperspecific category formation in autism is in some ways orthogonal to the loss of volitional control of top-down signals in psychosis. It remains theoretically possible, for example, for a person to have both high vigilance and impoverished volitional control. Yet the pertinent issue is whether or not the underlying genetic variation across individuals supports such phenotypes. One recent study examining speech discrimination in schizophrenia (Vercammen et al., in press) suggests that hallucinations may actually be associated with lower-than-normal vigilance levels, supporting the diametrical hypothesis of $\mathrm{C} \& \mathrm{~B}$, at least with respect to vigilance control. Detailed computational modeling of the autistic-psychotic spectrum, should it exist, may help to clarify the relationship between parameters underlying the brain's computational machinery and the genetic factors underlying the development of phenotypic variations in human cognition and behavior.

\section{ACKNOWLEDGMENTS}

I thank Stephen Grossberg for useful comments on an earlier version of the commentary. This work was supported in part by the National Science Foundation (NSF SBE-0354378).

\section{NOTE}

1. I am coining the phrase "autism-psychosis spectrum disorders" here to emphasize the unification of apparently diverse syndromes by a single theory.

\section{Digit ratio (2D:4D) as a marker for mental disorders: Low (masculinized) 2D:4D in autism-spectrum disorders, high (feminized) 2D:4D in schizophrenic-spectrum disorders}

\author{
doi:10.1017/S0140525X08004445
}

\author{
Martin Voracek \\ Department of Basic Psychological Research, School of Psychology, \\ University of Vienna, A-1010 Vienna, Austria. \\ martin.voracek@univie.ac.at
}

http://homepage.univie.ac.at/martin.voracek/

\begin{abstract}
Augmenting and supplementing the arguments of Crespi \& Badcock (C\&B), I show that digit ratio (2D:4D), a putative marker of prenatal androgen action, indeed appears differentially altered in autism-spectrum disorders (lower/masculinized) versus schizophrenicspectrum disorders (higher/feminized). Consistent with C\&B's framework, some evidence (substantial heritability, assortative mating, sex-specific familial transmission) points to possible sex chromosome and imprinted genes effects on 2D:4D expression.
\end{abstract}

Crespi \& Badcock $(\mathrm{C} \& \mathrm{~B})$ are to be congratulated for an outstanding theoretical contribution that may constitute a breakthrough regarding our understanding of autism-spectrum versus schizophrenic-spectrum disorders. Although their approach is theoretically guided, it is also data driven. That is, although supposed imprinted genes accounting for these disorders have yet to be pinpointed, C\&B amass a large body of evidence (see their target article's Table 1) showing that the disorder phenotypes are diametrically opposed. Therefore, it is legitimate and important to criticize and refute, or to augment and supplement, any constituents of their evidence put forward.

Inter alia, C\&B review some digit ratio (2D:4D) research findings consistent with their proposition (see sect. 7 on "Sex differences"). No less than four references (Arató et al. 2004; Manning et al. 2001; Milne et al. 2006; Walder et al. 2006a) from this brief passage are listed under their key references, so this clearly has relevance. In what follows, I augment and supplement further evidence from 2D:4D research consistent with the $\mathrm{C} \& \mathrm{~B}$ hypothesis.

2D:4D is the length ratio of the second digit (index finger, 2D) to the fourth digit (ring finger, 4D). This trait is sexually differentiated (men have lower 2D:4D than women) and may be a marker for the organizational (permanent) effects of prenatal androgen action (Manning et al. 1998). C\&B have missed some important further 2D:4D research references. This is fully excusable, but the gaps in the cited literature should be filled. As of this writing (early 2008), the 2D:4D literature numbers about 230 journal publications and about 50 unpublished theses, and is therefore overviewed only by specialists. Owing to the fast research progress, complete and topical reviews of 2D:4D research are currently unavailable (for partial reviews, see 
Cohen-Bendahan et al. 2005; Manning 2002; Peters et al. 2002; Putz et al. 2004; Voracek \& Dressler 2007; Voracek \& Offenmüller 2007; Voracek \& Pavlovic 2007; Voracek et al. 2006; 2007).

Ten studies (C\&B cite two: Manning et al. 2001; Milne et al. 2006) are informative for 2D:4D in autism-spectrum disorders. Manning et al. (2001) found that children with autism or highfunctioning autism (Asperger syndrome) and their first-degree relatives (unaffected siblings, mothers, and fathers), had markedly lower (hypermasculinized) 2D:4D than healthy population controls. This basic finding replicated in a study of autistic patients from Japan (Osawa et al. 2005), was also replicated by Milne et al. (2006) and de Bruin et al. (2006) for boys with autism, but only partially replicated by Falter et al. (in press) for boys with autism, who had somewhat, but not significantly, lower 2D:4D than normals. Knickmeyer et al. (2006) mention data indicating that girls with autism as well show masculinized (low) 2D:4D. Relatedly, three further papers have reported associations of low 2D:4D with various attention-deficit/hyperactivity disorder (ADHD) subtypes in children (Martel et al., in press; McFadden et al. 2005; Stevenson et al. 2007). Of further note, Voracek and Dressler (2006) have found no correlation of 2D:4D with autistic-like personality traits and other relevant dimensions (empathizing and systemizing) in a large healthy general population sample.

Five studies (C\&B cite two: Arató et al. 2004; Walder et al. 2006a) are informative for 2D:4D in schizophrenic-spectrum disorders. Arató et al. (2004) focused on distal finger-extent patterns (2D and 4D, both relative to 3D) rather than on 2D:4D and ascertained a smaller distance from $2 \mathrm{D}$ tip to $3 \mathrm{D}$ tip and a larger distance from $4 \mathrm{D}$ tip to $3 \mathrm{D}$ tip (i.e., relatively longer $2 \mathrm{D}$ and relatively shorter $4 \mathrm{D}$, both broadly equivalent to a high, feminized 2D:4D) in both male and female schizophrenic patients, compared with normals. Walder et al. (2006a), using a similar method, reported feminized distal finger extent in male (but not female) adolescents with schizotypal personality disorders, compared with healthy controls. The results of another study (Procopio et al. 2006) are difficult to interpret, as an uncommon measure (height $[h]$ divided through $2 \mathrm{D}$ or $4 \mathrm{D}$ ) was used, which evidently was confounded by patient-control differences in height. Female (but not male) schizophrenic patients had significantly higher $h: 2 \mathrm{D}$ than controls. Weißegger (2004) found markedly higher 2D:4D in female schizophrenic patients than in controls. However, the effect was, due to sample size limitations, statistically not significant, though noticeably smaller in males; and a follow-up study (Baumhackl 2007) failed to replicate any 2D:4D differences between schizophrenic patients and normals.

On the whole, the literature suggests 2D:4D to be low (masculinized) autism-spectrum disorders and high (feminized) in schizophrenic-spectrum disorders. An earlier conjecture (Manning 2002, p. 143), namely that 2D:4D in schizophrenia, as in autism, could be low (male-typed), appears untenable. The evidence is not without inconsistencies and replication failures, and appears stronger and more unequivocal for autismspectrum than for schizophrenic-spectrum disorders. Whereas associations of 2D:4D and autistic-like traits apparently do not generalize to the normal population (Voracek \& Dressler 2006), it remains to be seen whether associations of 2D:4D and schizotypal traits do. Additional 2D:4D data pertaining to both autism and schizophrenia would certainly benefit tests of the C\&B hypothesis.

Also worth consideration is the substantial heritability $\left(h^{2}=.60\right.$ to .80$)$ of $2 \mathrm{D}: 4 \mathrm{D}$ (twin studies: Gobrogge et al. 2008; Paul et al. 2006; Voracek \& Dressler 2007; family studies: Dressler et al. 2004; Marshall 2000; Ramesh \& Murty 1977), along with evidence for assortative mating on the trait (spousal correlations: about $r=.20$; Voracek et al. 2007). Further, 2D:4D is a correlate of androgen sensitivity, as shown through correlations with functional polymorphisms of the androgen receptor gene, located on the $\mathrm{X}$ chromosome, in men (Manning et al. 2003). Surprisingly,
2D:4D family studies findings seem more consistent with hypothetical Y-linked inheritance (i.e., strong father-son and brother-brother correlations) than with X-linked inheritance, but identification of Y-linked inheritance is known to be elusive. All of this points to possible direct sex chromosome and imprinted gene effects on 2D:4D expression. If so, this in turn would give clearer support of the C\&B hypothesis, as far as $2 \mathrm{D}: 4 \mathrm{D}$ is concerned. It may also shed more light on further intriguing differences (e.g., age of onset, sex ratio of prevalence) between autism-spectrum and schizophrenic-spectrum disorders.

\section{Authors' Response}

\section{The evolutionary social brain: From genes to psychiatric conditions}

\author{
doi:10.1017/S0140525X08004457
}

\section{Bernard Crespi ${ }^{\mathrm{a}}$ and Christopher Badcock ${ }^{\mathrm{b}}$}

${ }^{a}$ Department of Biosciences, Simon Fraser University, Burnaby, BC V5A 1S6, Canada; ${ }^{\mathrm{b}}$ Department of Sociology, London School of Economics, London WC2A $2 A E$, United Kingdom.

crespi@sfu.ca

C.Badcock@Ise.ac.uk

http://www.sfu.ca/biology/faculty/crespi/

http://www.Ise.ac.uk/collections/sociology/whoswho/badcock.htm

Abstract: The commentaries on our target article, "Psychosis and Autism as Diametrical Disorders of the Social Brain," reflect the multidisciplinary yet highly fragmented state of current studies of human social cognition. Progress in our understanding of the human social brain must come from studies that integrate across diverse analytic levels, using conceptual frameworks grounded in evolutionary biology.

We thank the commentators for providing insightful and constructive input on our target article, and for pointing out topics that will benefit from additional discussion. Our response is structured along the same general lines as the target article, scaffolding up from genes to cognition, behavior, and psychiatric conditions of the social brain. We first clarify some key aspects of the genetic and epigenetic bases of our theory, by describing the patterns and processes of genomic imprinting in more detail (sect. R1), and by showing how not just human imprinting-related syndromes, but also neurogenetic syndromes mediated by losses versus gains of suites of non-imprinted genes, contribute to diametric autistic versus psychotic phenotypes (sect. R2). Next, we show how phenotypes associated with growth and mentalistic or mechanistic skills can serve to connect genes and development with cognition and behavior (sect. $\mathrm{R} 3$ ), and we address the core issue of cognitive and behavioral similarities (sect. R4) and differences (sect. R5) between psychotic- and autistic-spectrum conditions. We focus, as did many of the commentators, on the degree to which these conditions can be considered as opposite quite literally, opposing in their developmental bases and diametric in their cognitive phenotypes, reflecting biases towards mentalism or mechanistic thought. Finally, we explain in greater detail how imprinting effects are expected to interact with sex differences, in generating the considerable diversity of autistic-spectrum and psychotic-spectrum conditions that we observe in nature (sect. R6), and provide closing thoughts (sect. R7). 


\section{R1. Mechanics of genomic imprinting}

\section{R1.1. Theory}

We are grateful to David Haig for providing detailed discussion of how natural selection is expected to drive the evolution of imprinting effects on phenotypes, given the diversity of fitness-mediating interactions between various asymmetric kin. We have focused on motherchild interactions, both physiological and behavioral, because these represent the best understood, and most clearly expected, selective arena for genomic conflicts underlain by imprinting.

\section{R1.2. Number and action of imprinted genes}

The efficacy of Haigian imprinted gene effects on development, brain, and behavior is to some degree a function of the numbers of imprinted genes in humans (Keller) and how they may be subject to dysregulation (Davies \& Isles). The OTAGO database of imprinted genes (Glaser et al. 2006) lists 63 confirmed imprinted genes in humans plus 41 genes that are imprinted in mice with no data as yet from humans, and bioinformatic predictions from Luedi et al. (2007) suggest the presence of an additional 156 human-imprinted genes. These known or predicted imprinted genes are primarily protein-coding. Recent studies of small non-coding RNAs have also provided evidence for about 200 imprinted RNA genes in humans, many of them brain-specific in expression and some known to mediate neurodevelopmental disorders (Fiore et al., in press; Mattick \& Makunin 2006; Royo et al. 2006). Thus far, the identification and characterization of imprinted genes in humans has been a haphazard affair, but new genomic technologies (Gimelbrant et al. 2007) should provide us with a complete list within several years. Each and every brain-imprinted gene on this list should, in our view, be immediately suspected of engagement in genomic conflicts. Such conflicts will be expressed in neurocognitive development to the extent that an imprinted gene has successfully shifted the balance of genetic power in its own paternally biased or maternally biased interests, or is dysregulated via alterations to imprints or the machinery of imprint application, maintenance, or removal, in addition to alteration by mechanisms common to all genes, such as point mutations and forms of epigenetic modification independent of imprinting effects. This is all that we mean by dysregulated in "more ways" than non-imprinted genes (Davies \& Isles); none of the molecular mechanisms involved are known to be unique to imprinting, but there is a greater overall scope for alteration, and greater effects because such genes are effectively haploid.

Given the central role of a small number of imprinted genes, such as CDKN1C, in orchestrating placental development (Andrews et al. 2007), and their coordinated expression in complex networks of imprinted and nonimprinted genes that jointly regulate development (Varrault et al. 2006), the actual number of imprinted genes is much less relevant than their "extended genotypes," whereby imprinted genes regulate the expression of other genes. For example, the imprinted non-coding RNA miR-134 regulates LIMK1, a focal gene in the etiology of Williams syndrome (Järvinen-Pasley et al. 2008), and imprinted-gene effects appear to be pervasive in key networks underlying development (Smits \& Kelsey 2006).

\section{R1.3. Roles of imprinted and non-imprinted genes}

How important are imprinted genes in neurodevelopmental disorders, compared to non-imprinted genes (Deutsch, Ludwig, \& McIlvane [Deutsch et al.], Dickins, Dickins, \& Dickins [Dickins et al.], Fitzgerald \& Hawi, Keller)? We reiterate that autistic- and psychotic-spectrum conditions are each highly heterogeneous in their etiologies, though convergent in core phenotypes, and that our hypothesis posits a phenotypic diametricity for these conditions that is underlain in part by the genomic diametricity of imprinting. In addition to the genetic evidence discussed in our target article, we also note that: (1) autism induced by uncontrolled PKU deficiency (Dennis et al. 1999) may be mediated by strong overexpression of the paternally expressed imprinted gene NNAT (Surendran et al. 2005); (2) overgrowth and autism caused by NF1 gene alterations may be driven by a paternal-allele-specific interaction with the imprinted IGF2-H19 locus (Ling et al. 2006); and (3) recent evidence implicates dysregulated processing of non-coding RNAs (which may include imprinted RNAs) in Fragile X syndrome (Plante et al. 2006), as also suggested by studies of the Prader-Willi phenotype found at low frequency in patients with the Fragile $\mathrm{X}$ full mutation but no epigenetic modifications to chromosome 15 (Nowicki et al. 2007). All of these cases require further study, but taken together with the evidence described further on in this response and in our target article, they indicate that alterations to imprinting represent a pervasive, convergent cause of many manifestations of autism, though not an exclusive one (Happé et al. 2006). We are aware of no alternative genetically based theory that can at least potentially account for such a broad and deep range of evidence or for such otherwise-inexplicable etiologic heterogeneity.

Of course, many non-imprinted genes have been linked with autism, as well as with schizophrenia, in genome scans and association studies - each with small effects on risk that have been difficult to replicate among studies (e.g., Craddock et al. 2005). Imprinted genes are necessarily embedded in physiological and developmental networks, so such important contributions to neurodevelopmental disorders from non-imprinted genes are, as noted by Keller and described in Keller and Miller (2006), by no means unexpected. But consider the relative strength of imprinted-gene effects, in each case for one or several genes: (1) the risk of psychosis rises to near $100 \%$ in Prader-Willi syndrome due to maternal uniparental disomy, which involves combined loss of paternal-gene expression and increased maternal-gene expression (Soni et al. 2008); (2) loss of function of the paternally expressed SGCE gene leads to high rates of psychotic and depressive symptoms (Lauterbach et al. 1994; Yokoi et al. 2006); and (3) loss or reduction of functional gene product of the maternally expressed UBE3A gene causes Angelman syndrome and is also associated with Rett syndrome, with both syndromes involving very high rates of autism and striking phenotypic overlap for diverse traits (Jedele 2007); UBE3A has also been implicated in idiopathic autism (Samaco et al. 2005). Similar considerations apply 
to the gene MeCP2 that directly underlies Rett syndrome: it exhibits allelic variants linked with idiopathic autism (Shibayama et al. 2004), and its functions include interaction with imprinted genes (Nomura et al. 2008).

As noted by Haig and Davies \& Isles, maternal duplications of the 15q11-q13 region have also been associated with autism; however, genotype-phenotype correlations are extremely variable for cytogenetic alterations of this region (here, interstitial duplications and supernumerary duplicated-chromosome fragments, referred to as idic15) (Cohen et al. 2007; Kwasnicka-Crawford et al. 2007). For example, Prader-Willi phenotypes have been found in individuals with paternally inherited interstitial duplications of 15q11-q13 (Engelen et al. 1999; Mao et al. 2000), and supernumerary-chromosome cases, all of which are maternally derived, involve severe developmental delay and phenotypes that have been characterized as "atypical" autism (Cohen et al. 2007; Cook et al. 1997). Studies analyzing gene expression and effects from all candidate genes in this region, which include GABRB3 (Dykens et al. 2004) and the FMRP-interacting gene CYFIP1 (Nishimura et al. 2007b; Nowicki et al. 2007) as well as UBE3A (which shows increased expression in idic15 cell lines; Baron et al. 2006), are required for robust interpretation of these alterations, given the complexity of the genetic and epigenetic regulatory networks involved (e.g., Landers et al. 2005). By contrast, Angelman syndrome has a simple, well-documented cause: reduced expression or activity of the maternally expressed gene UBE3A.

Our hypothesis can also not be evaluated directly from information on the expected or observed parental expression patterns of specific imprinted genes associated with autistic- or psychotic-spectrum conditions (Venkatasubramanian), because we require information on the direction of change in gene-expression levels or geneproduct activity. For example, allelic variation in several genes, including DISC1 (Chubb et al. 2008; Kilpinen et al. 2008), CNTNAP2 (Arking et al. 2008; Friedman et al. 2008), UBE3A (Nurmi et al. 2003; Sporn et al. 2004b), RELN (Serajee et al. 2006; Shifman et al. 2008) and HLA-DRB1 (Torres et al. 2002; Wright et al. 2001), has been associated with both autism and schizophrenia; do these genes exhibit diametric alterations or expression patterns in the two conditions, in some cases mediated by imprinting? UBE3A is known to be imprinted, DISCl is not imprinted despite a bioinformatic prediction (Hayesmoore et al., in press; Luedi et al. 2007), and CNTNAP2 and RELN show parent-of-origin effects in their associations with autism (Arking et al. 2008; Dutta et al. 2007; Rampersaud et al. 2007). Tordjman describes salient evidence from the SLC6A4 gene, of alternative alleles associated with more-impaired sociality in autism versus severity of auditory hallucinations in schizophrenia, that provides a clear example of the types of genetic data most useful in evaluating our hypothesis.

Taken together, these studies indicate that dysregulation of imprinted genes exerts strong, penetrant effects on the expression of autistic- and psychotic-spectrum conditions that are generally in the directions predicted by our hypothesis. Linkage-scan and genetic-association studies usually do not test for parent-of-origin effects, or quantify epigenetic variation, so the degree to which such major effects are paralleled by smaller, much more numerous influences, remains unexplored.

\section{R2. Genomic copy-number variation and diametric syndromes}

Our hypothesis is more general than imprinted gene effects, in postulating the evolution of human neurocognitive architecture along a major axis from mechanistic to mentalistic cognition, manifested in psychiatric pathology by autistic and psychotic spectrum conditions. Such architecture is expected to canalize genotype-phenotype associations for diverse forms of genetic, epigenetic, and genomic variation. Burne, Eyles, \& McGrath (Burne et al.), Deutsch et al., and Keller draw attention to gene copy number variation as a recently characterized source of human genomic variability, one that provides opportunities for novel tests of our hypothesis.

\section{R2.1. Copy number variation in neurogenetic syndromes}

The best-known cases of copy-number variation to date involve neurogenetic syndromes caused by deletions versus duplications of autosomal, non-imprinted regions harboring multiple genes, or deletions versus duplications of the entire X chromosome itself. For autosomes, deletions cause haploidy for genes in the region, whereas duplications result in triploidy. Do these diametric genomic alterations result in diametric neurocognitive phenotypes, in particular autistic- versus psychotic-spectrum phenotypes? We have summarized salient, available information for three of the best-understood pairs of autosomal neurogenetic syndromes in Table R1. In each of these three cases, one of the syndromes is notably associated with psychotic spectrum diagnoses and behavior (as also summarized, e.g., by Table 2 in Gothelf [2007]), while its sister syndrome (Crespi, in review) is associated with autistic-spectrum diagnoses and behavior. We also highlight the observation that the three conditions considered here as falling into the psychotic spectrum, Smith-Magenis syndrome, Williams syndrome, and velocardiofacial syndrome, are each characterized by higher verbal than visual-spatial skills, and by undergrowth, as also reported for Prader-Willi syndrome disomy (Dykens 2002; Roof et al. 2000; Whittington \& Holland 2004); similar considerations apply to schizotypy and psychosis, as documented in our target article. These findings provide notable support for a psychotic-autistic neurogenetic continuum underlying human cognition, a spectrum that should also be genetically mediated by myriad other, smaller-scale cases of copy-number variation throughout the human genome.

Neurogenetic conditions caused by "copy-number" variation of whole sex chromosomes require special consideration, because as Haig (2006) explains, genes on the $\mathrm{X}$ chromosome are expected to favor maternal interests and thus resemble maternally expressed imprinted genes in the nature of their predicted effects on development. As a result, by our hypothesis, Klinefelter syndrome (usually 47,XXY) should involve high rates of psychotic spectrum disorders, whereas Turner syndrome (usually $45, \mathrm{X})$ should involve a higher incidence of autism. As also noted in our target article and shown in Table R1, this prediction is met (Boks et al. 2007a; DeLisi et al. 2005; Skuse 2000; 2005). Based on these considerations, we also make the a priori prediction of high rates of 
Table R1. Neurogenetic sister syndromes mediated by genomic copy-number variation can be categorized as involving cognitive, behavioral, and psychiatric phenotypes characteristic of either the psychotic spectrum (left column) or autistic spectrum (right column).

Where relatively little directly salient data are available (as for Smith-Magenis syndrome and velocardiofacial syndrome-region duplications), we predict that, upon further study, the syndromes will engender phenotypes and psychiatric diagnoses characteristic of the relevant spectrum. Crespi (in review) provides a comprehensive evaluation of the degree to which these and other neurogenetic sister syndromes involve diametric phenotypes

Smith-Magenis syndrome

Caused by hemizygous deletion of region at 17p11.2 including

RAI1 gene, and by RAI1 mutations (1)

Undergrowth $(1,2)$

Case report of mood disorder (5)

Relative strength in verbal skills (6)

Case reports of "bipolar episodes" (7)

\section{Velocardiofacial syndrome}

Caused by deletion of over 30 genes at 22q11.2, effects from COMT, DGCR2, TBX1, other genes $(9,10)$

Undergrowth (11)

Better verbal than visual-spatial skills $(12,13)$

High rates of schizophrenia, schizoaffective disorders, bipolar, depression, anxiety, phobia (25-30 fold increase) (9)

\section{Potocki-Lupski syndrome}

Caused by duplication of same region (8)

Low birth weight but not short stature, poor feeding in infancy (8) Seizures, hyperactivity (8)

High rates of autism and autistic behavior (in "vast majority of cases") (8)

\section{Velocardiofacial-region duplications}

Caused by duplication of same region (14)

Poor growth in $7 / 12$ cases (14)

Hyperactivity, attention deficits, anxiety, depression, seizures, speech problems, impulsivity (14-16)

Case report of autism (17)

Case report of Asperger syndrome (18)

Two cases of autism (19)

No duplications in 190 schizophrenia patients (20)

\section{Williams syndrome}

Caused by deletion of over 25 genes at $7 q 11.23$; effects from CYLN2, GTF2I,GTF2IRD1,LIMK1 (21-24)

Undergrowth (25)

Feeding difficulties (26)

Better verbal than visual-spatial skills $(21,27)$

Hypersociability, fascination with faces (26)

High rates of anxiety and phobias (over $50 \%$ of cases) (29,30); presence of autistic traits is controversial (31)

\section{Williams-region duplications}

Caused by duplication of same region (32)

Growth highly variable, but head size appears relatively large (32)

Seizures (32)

Severe language impairment, visual-spatial skills spared (32)

Very high rates of autism and autistic behavior $(32,33)$

\section{Klinefelter syndrome}

Usually caused by $47, \mathrm{XXY}$, in males; increased expression of pseudoautosomal-region 1 genes and other non-X-inactivated genes $(34,35)$

Low birth weight (36)

Dyslexia (37)

Poor verbal skills, preserved visual-spatial skills $(35,38)$

High rates (4- to 10-fold increase) of schizophrenia; also high rates of schizoaffective disorder schizotypy, bipolar, anxiety, depression $(35,39,40)$

\section{Turner syndrome}

Usually caused by 45,X, in females; haploinsufficiency of pseudoautosomal-region 1 genes $(41,42)$

Low birth weight, adult stature $(43,44)$

Hyperlexia (45)

Good verbal skills, impaired visual-spatial skills $(38,44)$

Gaze aversion (46)

High rates (over 100-fold increase) of autism and autistic traits with intact X maternally derived $(47,48)$

Reduced incidence of schizophrenia plus bipolar (49)

Table R1 References. The most recent and most comprehensive references are provided here, and more complete documentation can be found in the reference lists of these publications: (1) Gropman et al. (2007), (2) Smith et al. (2005), (3) Sarimski (2004), (4) Smith et al. (1998), (5) Bersani et al. (2007), (6) Dykens et al. (1997), (7) Girirajan et al. (2006), (8) Potocki et al. (2007), (9) Gothelf (2007), (10) Meechan et al. (2007), (11) Weinzimer et al. (1998), (12) Bearden et al. (2001), (13) Lajiness-O'Neill et al. (2006), (14) Ensenauer et al. (2003), (15) Yobb et al. (2005), (16) Alberti et al. (2007), (17) Mukaddes \& Herguner (2007), (18) Hassed et al. (2004), (19) Marshall et al. (2008), (20) Brunet et al. (2008), (21) Tassabehji (2003), (22) Gray et al. (2006), (23) Edelmann et al. (2007), (24) Young et al. (2008), (25) Martin et al. (2007), (26) Sugayama et al. (2007), (27) Vicari et al. (2004), (28) Doyle et al. (2004), (29) Dykens (2003), (30) Leyfer et al. (2006), (31) Lincoln et al. (2007), (32) Berg et al. (2007), (33) Depienne et al. (2007), (34) Vawter et al. (2007), (35) DeLisi et al. (2005), (36) Ratcliffe et al. (1990), (37) Geschwind et al. (2000), (38) Money (1993), (39) van Rijn et al. (2005), (40) Boks et al. (2007), (41) Zinn et al. (2007), (42) Lynn \& Davies (2007), (43) Wisniewski et al. (2007), (44) Kesler (2007), (45) Temple \& Carney (1996), (46) Lawrence et al. (2003), (47) Skuse et al. (1997), (48) Crespi (in press b), (49) Mors et al. (2001). 
autism in Simpson-Golabi-Behmel syndrome, which is caused by loss of function of the X-linked GPC3 gene and involves prenatal and postnatal overgrowth (Rodríguez-Criado et al. 2005). Fragile X syndrome also, of course, involves loss of function for a key X-linked gene, as well as overgrowth and high rates of autism.

\section{R2.2. Angelman and Prader-Willi syndromes}

Angelman and Prader-Willi syndromes are, like BeckwithWiedemann and Silver-Russell syndromes, "sister syndromes" mediated by diametric alterations to imprinted regions, in the same general way that the sister syndromes described earlier are mediated by diametric alterations to gene copy number for a genomic region. The main difference is that for imprinted regions, gene expression can be doubled or lost for the affected regions, as opposed to alterations to a haploid or triploid state for autosomal non-imprinted genes. Both forms of variation are the closest we will ever achieve to human equivalents of mouse models (such as gene knockouts), which have provided many key insights into developmental mechanisms, as described by Burne et al.

Several commentators have raised Angelman and Prader-Willi syndromes to rather exalted status in the exposition and support of our theory, calling them a "cornerstone" (Davies \& Isles), "central dogma" (Fitzgerald \& Hawi), or "conceptual core" (Dickins et al.). We consider them not nearly so central or crucial, but Prader-Willi syndrome in particular has provided us with clear insights into linkages among imprinted genes, neurological function, behavior, and psychiatric disorders (Haig \& Wharton 2003; Crespi, under revision). For example, Prader-Willi syndrome teaches us about how multiple genetic and epigenetic causes can generate the same or a very similar phenotype: Hence, this syndrome (or most of its major features) has been associated with a large suite of different independent genetic and epigenetic causes, many of which are known or believed to involve imprinted genes, hypothalamus dysfunction, or both (Crespi, under revision). Now imagine such Prader-Willi genetic deviations and phenotypes on a much smaller scale, and bereft of pathology: a small, complacent baby with gentle suck and little cry, changing upon weaning to a relatively independent child who seeks his or her own food (Haig \& Wharton 2003), and perhaps also engages in other maternally undemanding pursuits, such as jigsaw puzzles (Dykens 2002). We have posited a smooth genetic and phenotypic continuum between an easy child physiologically and socially and, in extrema, a child who, like one with Prader-Willi syndrome, matures to face psychosis and depression. Ultimately, as Haig suggests, the genes will decide - especially, we might add, genes expressed in the brains of fetuses and young children.

\section{R3. Integrative analyses of neurodevelopmental disorders}

The diversity of approaches represented by the various commentators is truly impressive, and it demonstrates the utility of, for example, evolutionary theory (Haig), computational biology (Vladusich), animal genetics and physiology (Burne et al., Davies \& Isles), linguistics
(Frawley), neuroscience (Oberman \& PascualLeone), psychology (Kanazawa, Langdon \& Brock, Nettle, Thakkar, Matthews, \& Park [Thakkar et al.], Troisi), and clinical psychiatry (Behrendt, Goos) in understanding human neurodevelopmental disorders. The cost of such disciplinary specialization, of course, is blindness to the whole conceptual and empirical elephant, an elephant that must be constructed in toto by stitching parts together to see how well they fit.

These disciplines can be synthesized using the conceptual tools of endophenotypes, intermediate phenotypes, and behavioral phenotypes (Cannon \& Keller 2006; Feinstein \& Singh 2007; Meyer-Lindenberg \& Weinberger 2006), whereby traits or mechanisms characteristic of a given psychiatric condition serve as guides for analyses downwards to genes and upwards to cognition and behavior. Goos encourages an increased focus on endophenotypes involving social skills to evaluate imprinting effects - we concur, but note that mother-offspring interactions involve much more than social skills per se; indeed, hyperactivity and disrupted sleep impact upon mothers of children with Angelman syndrome (Barry et al. 2005), and most actions of infants and children, social or not, are likely to affect levels of cost imposed on mothers. To evaluate social and nonsocial interactions, and their psychological mechanisms, Troisi stresses the ecological validity of the tests involved; we agree, citing mechanistic gaze-following skills of autistics, which can be mistakenly interpreted as mentalistic, as a possible example (Langdon \& Brock) (e.g., Nation \& Penny 2008; Senju et al. 2004; see also Chiu et al. 2008). The key "ecology" here for imprinted genes is costs and benefits to mother and to child, which have yet to serve as a focal criterion for psychiatric-behavioral research.

In this analytic context, we reiterate that our hypothesis predicts alterations in physiology and behavior that are always adaptive (to either mother or offspring) only for small deviations in development, cognitive architecture, and behavior - the larger the deviation, the harder it becomes to separate adaptation from pathology. The continuum from dynamic balance, to one-sided adaptations, and then to pathology in either direction, is illustrated in different ways in Figures 3,4, and 5 of the target article, which may help to address the concerns of Langdon \& Brock and Thakkar et al. regarding the predicted mentalistic abilities of schizophrenics, described in more detail later in section R6.2 on hypermentalism. This continuum extends to the molecular level - for example, the unexpected association between in utero growth restriction and paternal over-expression (due to duplication) at 6q24 (Temple \& Shield 2002) highlighted by Dickins et al. is likely a pathological effect (Temple, personal communication); the mouse model for this situation, a knockout of the paternally expressed, imprinted Zacl gene, shows the pattern of growth restriction predicted by the conflict theory (Varrault et al. 2006). Separating adaptation from pathology in this context, and making robust falsifiable predictions, requires sufficient knowledge of molecular-developmental mechanisms. As for skepticism regarding the conflict theory itself (Dickins et al.), we refer readers to Table 1 of Fowden et al. (2006), which nicely summarizes support in the only context analyzed comprehensively to date, placentation. 
Does our hypothesis provide insights into specific, core phenotypes characteristic of autistic and psychotic spectrum conditions, bearing in mind that the heterogeneity in each spectrum may preclude universal generalizations (Davies \& Isles, Deutsch et al.) and that mentalizing functions exhibit considerable diversity (Goos, Langdon \& Brock, Troisi)? Psychotic spectrum conditions appear to almost universally involve better verbal skills than visual-spatial skills, and indeed this imbalance has been suggested as an endophenotype for schizophrenia (Kravariti et al. 2006). The only obvious exception of which we are aware is Klinefelter syndrome (Table R1), which is unique in involving X-chromosome imbalance. In keeping with this pattern, we predict better verbal than visual-spatial skills in bipolar disorder and major depression, as evidenced by the literary and artistic prowess of some individuals with these conditions described further on, but apparently not yet tested with robust psychological tools. Some verbal skills can be mechanistic, as seen in Asperger's syndrome and autistic hyperlexia, but to the extent that social verbal abilities form a core of mentalistic cognition, this pattern fits well with an autistic-psychotic continuum.

The primary phenotype common to autistic-spectrum conditions is more or less temporary childhood overgrowth for metrics of body size, head size, or both, being found in idiopathic autism (Hobbs et al. 2007; Mraz et al. 2007; van Daalen et al. 2007; Webb et al. 2007; Xiong et al., in press; and citations in target article), and in the autistic conditions Fragile X syndrome (Chiu et al. 2007; Terracciano et al. 2005), PTEN mutations (Butler et al. 2005), RNF135 mutations (Douglas et al. 2007), and 22q13 deletion syndrome (Bonaglia et al. 2006). Sotos and Weaver overgrowth syndromes also show notable evidence of autistic behavioral phenotypes (de Boer et al. 2006; Derry et al. 1999; Mouridsen \& Hansen 2002; Rio et al. 2003; Sarimski 2003; but see also Compton et al. 2004), and tuberous sclerosis may also involve macrocephaly (Fidler et al. 2000). The main exceptions here are Angelman and Rett syndromes, although a mouse model of Angelman syndrome shows some evidence of general overgrowth (Johnstone et al. 2006). The perceptual, developmental, and computational perspectives of Belmonte, Oberman \& Pascual-Leone, van Rijn, Swaab, \& Aleman (van Rijn et al.), and Vladusich provide insights into potential links between early brain or general overgrowth, increased local to global processing (or increased bottom-up compared to top-down processing), and autistic cognition. The links of overgrowth to a paternal bias in imprinted gene expression have been amply demonstrated in mouse models, and in some human genomic conditions such as Beckwith-Wiedemann syndrome, which has - since after completion of our target article - also been demonstrated to involve an elevated incidence of autism (Kent et al., in press).

Our theory is perhaps least developed thus far in drawing connections between mechanistic versus mentalistic cognition and phenotypes, such as growth and behavior, that impose more rather than fewer costs on the mother. For example, as described by Dickins et al., Goos, and Troisi, selfish behavior can be mentalistic or mechanistic, perhaps associated in psychopathy with quite different sources of social isolation and deficits in empathy. A role for imprinting effects in selfish, egocentric behavior per se should be restricted to mother-offspring interactions (or other classes of asymmetric relative, though less clearly; see Haig) - but once such a brain develops in childhood, as in Asperger syndrome, it cannot be expected to change fundamentally with increasing age. The clearest connections between mechanistic cognition and selfishness may be tendencies in autism to treat people as "things," and to develop nonsocial skills in science and engineering (Badcock 2008; Wheelwright \& Baron-Cohen 2001). But are healthy children who are "easy" on mother also more mentalistic? Nettle's hypothesis that more mentalistic mates may, like a Williams-syndrome child, be more empathic, sensitive, and solicitous to one's wants and needs may translate into a mother-offspring context, although we still expect parent-offspring conflicts mediated by non-imprinted genes.

\section{R4. Similarities between autistic- and psychotic- spectrum conditions}

Our exposition of autistic- and psychotic-spectrum conditions as diametric has raised the issue of their apparent similarities, a topic that traces back to Bleuler's coinage of "autism" as a facet of schizophrenia. Thus, Burne et al. note that paternal age and prenatal infections influence both autism and schizophrenia risk; Davies \& Isles describe how attention and inhibitory processes are dysfunctional in both sets of conditions; and Venkatasubramanian references papers on similarities between autism and schizophrenia in IGF-1 levels, digit ratios, and corpus callosum size. Aside from phenotypes involving physiological and morphological traits, which must be decided based on weight and quality of all the evidence (as we have attempted, and as Voracek kindly provides for digit ratios), evaluation of similarities must focus on whether they are only apparent, or, instead, reflect underlying similarities in etiology that stretch from genes to neurodevelopment and cognition.

\section{R4.1. Paternal age}

Paternal-age effects on autism and schizophrenia risk have indeed been well documented (Cantor et al. 2007; Laursen et al. 2007), and diverse evidence suggests that dysregulation of imprinted genes, which results in either a maternal or paternal expression bias, may mediate such links (Crespi 2006; Perrin et al. 2007; Reichenberg et al. 2006). Direct tests, involving quantification of how genomic, genetic, and epigenetic changes increase with age in sperm cells, are required to evaluate this matter further.

\section{R4.2. Infection and intrauterine growth}

The influences of prenatal maternal infection on brain development, and risk of schizophrenia or autism (Burne et al.), are strongly affected by the timing of infection during pregnancy (Mednick et al. 1994; Meyer et al. 2007). Based on these data, we expect that differences in the timing of prenatal infection, for the same infectious agent, should differentially affect the risk of autism and schizophrenia; for example, first-trimester infections may predispose to autism, while the same infection in the 
second trimester may increase schizophrenia risk (Fatemi et al. 2008). Prenatal resource deprivation should, given our expectation of overgrowth in autism and undergrowth in schizophrenia, also differentially lead to increases in the latter condition. For example, a study of 2 million Swedish children born between 1963 and 1983 revealed a significant link between schizophrenia and poverty in childhood. Those with four out of five measured indicators of hardship had an almost threefold greater risk of schizophrenia than those with none (Wicks et al. 2005). Clearly, such findings would fit our paradigm of psychosis being a possible consequence of restricted growth and would predict that such developmental insults would not be found to predispose towards autism. Oberman \& Pascual-Leone suggest a role for a different form of environmental effect in autism: recent increases in sensory stimulation during childhood - might secular trends of increasing infant birth weight, due to higher nutrient levels, likewise be involved in recent apparent increases in rates of autism?

Our interpretation of psychosis being a consequence of enhanced maternal rather than paternal brain function would also fit the suggestion that schizophrenia may sometimes be caused by infection with the protozoan parasite Toxoplasma gondii (Webster 2007; Webster et al. 2006; Yolken \& Torrey 2008). People infected with T. gondii (between $15 \%$ and $80 \%$, depending on the population) are three times more likely to suffer from schizophrenia than those not infected, and so too are cat-owners. The significance of the latter may lie in the fact that the parasite can only complete the reproductive phase of its life-cycle inside a cat. It achieves this by causing its principal carriers, rats and mice, to lose their fear of cats, and so be much more likely to be eaten by one. Inside the rodent's brain, the parasite attacks the amygdala, which plays the same role in triggering fear reactions that it does in humans. But when infected rats are treated with anti-psychotic drugs like those given to human schizophrenics, the rats' fear of felines returns. Men with Toxoplasma infection tend to be more reckless than normal, and infected people of both sexes are almost three times more likely to be involved in car accidents, and have measurably slowed reaction times (Ginsburg 2004; Randerson 2002). The finding that in mice paternally expressed imprinted genes are differentially expressed in limbic system components like the amygdala and hypothalamus (Coan et al. 2005; Keverne et al. 1996) combined with the suspicions about $T$. gondii and schizophrenia in humans, suggests the intriguing possibility that an explanation may lie in the parasite suppressing paternal brain systems like the amygdala to produce an overall preponderance of maternal brain function, which we have argued could be a fundamental basis of psychosis in general and of schizophrenia in particular. Similar considerations may apply to cytomegalovirus, which has been reported to differentially affect the limbic system in humans (Yolken \& Torrey 2008).

\section{R4.3. Cognitive traits}

Cognitive, behavioral, and psychiatric similarities between autism and schizophrenia, such as attentional and inhibitory traits (Davies \& Isles) but especially negative symptom schizophrenia (Thakkar et al., Tordjman, van Rijn et al.) are, we believe, apparent and superficial rather than indicative of any common underlying genomic and neurodevelopmental etiologies. Virtually all of these latter similarities are based on behavioral and psychological data, and we challenge critics to find any clear neurodevelopmental evidence connecting these two conditions. As described by Tordjman, all of the traits most characteristic of negative symptom schizophrenia, "such as affective flattening, alogia, avolition, apathy, anhedonia, and poor communication," involve absences of traits that can be attributed to pathology, which cannot be taken as strong evidence of common etiology. As regards the causes of apparent similarities, Järvinen-Pasley et al. (2008) cogently remark that "equivalent behavioral scores may mask distinctly different cognitive processes," and Skuse (2007) contends, with regard to autism, that "in the absence of independent evidence of aetiology, symptomatic phenotypes reflect primarily the characteristics of the instrument used to measure them." Thakkar et al. in particular motivate us to further discussion of negative symptom schizophrenia, in addition to our model for its presence as a pathological effect of highly dysregulated neurodevelopment (Fig. 6), mediated in part by imprinting. This model is supported, for example, by associations between the presence and severity of negative symptoms in schizophrenia and increased loss of gray or white matter (especially in the prefrontal and temporal lobes; e.g., Sanfilipo et al. 2000; Turetsky et al. 1995; Wible et al. 2001), in striking contrast to greater retention of gray matter with age in Asperger syndrome, compared to controls (McAlonan et al. 2002). Frawley describes an instructive case of how functionally similar deficits (here, in private speech development) can arise from fundamentally different mechanisms in autism and schizophrenia. We expect that analogous studies will successfully fractionate other superficially similar dysfunctions of the social brain into autistic versus psychotic etiologies.

Klinefelter, Prader-Willi, and velocardiofacial syndromes exhibit clear biological links with schizophrenia and other psychotic-spectrum conditions but essentially no biological links with autism. Nevertheless, this suite of neurogenetic conditions has been considered to belong to the autism spectrum (or at least to comprise autistic symptoms) as a direct consequence of interpreting negative symptom schizophrenia and autism as closely related conditions. We consider this perspective to set psychiatry back intellectually nearly one hundred years to the days of Bleuler, and to deny Kanner's and Asperger's revolutionary insights into autism as a condition fundamentally separate from schizophrenia.

Perspectives and research programs based on identifying and characterizing specific deficits provide a limited conceptual framework, as do psychological theories of autism or schizophrenia (e.g., Behrendt); what we ultimately need are ways to connect across levels, from genes to neurodevelopment, morphology, physiology, psychology and cognition.

\section{R5. Differences between autistic- and psychotic- spectrum conditions}

Fitzgerald \& Hawi are correct in saying that another article could be written on the similarities between what 
we are calling autistic- and psychotic-spectrum disorders, and we would be happy to write a commentary on such an article. But just as the similarities between males and females can hardly be expected to reveal the important biological fundamentals of sex differences, so such an article would not reveal the underlying mechanisms which, in our view, explain the essential differences between these disorders. Our commentators have touched upon a suite of such developmental, cognitive, behavioral, and epidemiological differences that uncover new diametric patterns salient to our hypotheses and provide novel insights into the causes of autistic- and psychotic-spectrum disorders.

\section{R5.1. Age of onset}

The concepts of hypo- and hyper-mentalism facilitate understanding of the general issue raised by Behrendt and van Rijn et al., that classical autism is a disorder with invariable onset in childhood, whereas schizophrenia is very much a late adolescent- or adult-onset one (with the childhood-onset schizophrenia mentioned by Tordjman being very rare, and even then appearing later than autism; Frith 1992; Jacobsen \& Rapoport 1998). The reason could simply be that no one could develop the characteristic hyper-mentalism of psychosis without first developing a more normal level of mentalism - evidently something which usually takes the whole of childhood and the greater part of adolescence to achieve. It has already been suggested that schizophrenics may have an intact ability to mentalize right up to their first breakdown (Frith 1992), and Langdon has pointed out that if children who later develop schizophrenia had failed to acquire a normal theory of mind, they would have come to the attention of clinicians long before the onset of their illness (Langdon 2005). Clearly, this is predictable if schizophrenic symptoms represent pathological extensions of mentalism far beyond the normal range. But if autistics are symptomatically hypo-mentalistic, this would imply that they had never completed the normal process of mental development, but stopped short long before at some point in childhood (or perhaps regressed back to it). In any event, autistic symptoms would show in childhood, and psychotic ones would not be seen fully developed until later - which is exactly what we normally find.

\section{R5.2. Fertility}

No one has questioned our expectation of lower fertility of parents with relatively autistic children, but Keller has requested additional exposition on data regarding fertility in schizophrenics and their first-order relatives. The primary upshot of this short review of evidence is that two studies demonstrate statistically significant higher fertility of sisters of schizophrenics, with one of these studies showing such an effect only for schizotypal sisters (Bassett et al. 1996; Haukka et al. 2003). As we and Keller also note, other studies show a mix of lower or equal fertility of sibs, and equal or higher fertility for parents, which may be attributed to either the effects that we posit, or, as we describe, to environmental consequences of sibship size. Combined, average brother-sister fertility (Haukka et al. 2003) is irrelevant, as are any other specific, absolute measures, because such studies provide only cross-sectional data broadly suggestive of long-term evolutionary processes (Crespi 2006). Additional demographic studies of fertility in sibs, parents, and offspring of schizophrenics that control for environmental effects are certainly required for robust tests, but we suggest that Type I error is unlikely to afflict two independent studies, both providing data that fit closely with our model of sex and fertility effects in psychotic-spectrum conditions. With regard to predictions from Kanazawa of offspring sex ratios produced by individuals on the autistic and psychotic spectra, we would be concerned about effects from stoppage on the autistic side biasing the data, as well as the converse - the bearing of daughters leading to increased family size, given their apparent lower costs of rearing (Rickard et al. 2007).

What is perhaps most interesting about the demographic data just mentioned is that, combined with data on lower birth weight and growth in schizophrenia, they dovetail so nicely with Nettle's hypothesis of female mate choice for "healthy" schizotypy. Thus, under both parent-offspring conflict theory and the conflict theory of imprinting, mothers (and maternal genes) are selected for the bearing of smaller, less-demanding offspring, which permits higher fertility. As Nettle suggests, there may be selection for females to choose mates on the basis of such traits in children - and for higher fertility. This hypothesis is speculative, but its components are falsifiable, and it gains plausibility from the strength of selection on mate choice and on maternal-offspring interactions.

\section{R5.4. Inner and private speech}

Frawley describes a striking new example of apparent diametric differences between autism and schizophrenia: by his hypothesis, inner speech stays inner in autism, and private speech is underdeveloped; whereas in psychosis, private speech becomes hyper-developed into elaborate social themes involving auditory hallucinations. Jones and Fernyhough (2007) point out that auditory hallucinations in schizophrenia exhibit precisely the same manifestations as private speech in typically developing young children, thus providing a clear explanation, convergent with ours, for their social-developmental forms and contents. These considerations of inner and private speech highlight the role of Vygotsky as an intellectual father of the social brain hypothesis, and indeed of parent-offspring conflicts (Crespi, in press a); for example, Bentall (2003a, p. 196) paraphrased Vygotsky (1962) in noting that:

During this stage [of early childhood], the child and adult are constantly issuing instructions or asking questions of each other so that, for the child, the whole process of speaking becomes bound up with attempts by the two parties to control each other's actions.

Crespi (2007) describes recent evidence that genes underlying the development of speech, as a sociality-manipulative organ of the social brain, may also be subject to effects from imprinting.

\section{R5.5. Religion}

We endorse Kanazawa's remarks about religion here and in other works (Badcock 2004; 2008; in preparation), and he points out an obvious but counterintuitive prediction 
implied by the contention that autistic pathology is diametrically opposed to hyper-mentalism. This is that autistics ought to be much less religiously, magically, and superstitiously minded than normal. Although few studies have been done, what is known about autistics' attitude to religion bears out this expectation. For example, in their analysis of the legal proceedings which provide much of the evidence for their retrospective diagnosis of Hugh Blair of Borgue (1708/9-65) as an autistic, Houston and Frith comment that:

It follows that a lack of theory of mind restricts a religious sense ... for this reason the question posed by the judges: "Did Hugh Blair have a sense of God?" can be interpreted as analogous to the question posed by modern psychologists: "Did Hugh Blair have a theory of mind?" (Houston \& Frith 2000, pp. 164-65)

These authors point out that the final verdict of the court implies that the judges did not believe that Hugh had the same sense of God as other members of the community, and they conclude that this matches their interpretation of Hugh having a diminished awareness of mental states

One of the very few studies that has considered the evidence for religious feelings in autistics concludes that religion in autism is entirely different from that in normal experience (Bering 2002). Because autistics have difficulty interpreting the meaning attached to social behavior and therefore probably cannot rely on a theory of mind to explain their experiences, their religious beliefs cannot attach to core representations of mental agency. The religious beliefs of people with autism could therefore be seen as sliding into conceptual slots provided by what we would term mechanistic cognition - even those, such as concepts of supernatural agents, that are traditionally relegated to what we would call mentalism. Consequently, supernatural agents such as God are perceived as behavioral rather than intentional agents (Bering 2002). For example, an account of the religious belief of one highfunctioning autistic observes that the person concerned, "Simply could not cope with spirituality when it was seen as the collective considerations of others... $[\mathrm{O}]$ wing to the specific nature of his cognitive and emotional impairments he had found it very difficult to engage either in the theological or the philosophical debate that occurred in the traditional construction of religious practice" (Isanon 2001, p. 75). Again, a mother of two autistic sons remarks that her boys "don't turn to God as a way of navigating life's difficulties," adding that when one of them "was (inappropriately) attending a mainstream primary school, his report simply said, 'Religious Knowledge: not applicable"' (Moore 2004).

According to our view, superstition is another expression of hyper-mentalism. But the fact that no less an anti-mentalist than B. F. Skinner once published a paper entitled, “Superstition' in the pigeon" (Skinner 1948), suggests that we might distinguish between superstitious behavior - which, if found in a pigeon could certainly be expected in autistics - and superstitious belief, which is what we would expect in more mentalistically minded people. Although no studies of autistic superstition have been done to our knowledge, our theory makes a clear prediction: As in the case of religion, autistics should show mainly superstitious behavior, with little or no mentalistic elaboration like that typically seen in articulate psychotics (Badcock, in preparation). Indeed, this might answer Fitzgerald \& Hawi's observation that individuals with high-functioning autism may show fixed and rigid beliefs reminiscent of delusions observed in psychosis. Clearly, the typical autistic tendency to stubbornly focus on narrow interests, to resist change, and to be impervious to criticism could explain the superficial similarity of such delusions to those of psychotics; but, to the extent that those delusions were superstitious in content, we would expect them to be more pigeon-like than paranoid in any true sense. No matter what the content, however, our theory predicts that the delusions of autistics should be much less mentalistically elaborated than those of psychotics.

Finally, we wholly concur with Kanazawa's point about women and religion, and would only add that the same is true of magical and superstitious thinking. For example, women usually show stronger belief than men in extrasensory perception (especially telepathy and precognition) and in superstition (specifically, in astrology, ghosts, psychic healing, and reincarnation) (Irwin 1993). Selftranscendence is one well-validated personality measure of mystical tendencies on which women have been found to score 18\% higher than men (Hamer 2004, p. 36), and many other measures show a comparable sex difference in attitudes towards the supernatural. In the words of Nick Humphrey, the explanation may be that "it is women who arguably have the greater sensitivity to human personal relationships - and who, as every contemporary survey shows, tend to be surest that the scientific picture of the world is incomplete" (Humphrey 1996, p. 51). If we add that science is incomplete because it is notably hypo-mentalistic - and even anti-mentalistic - we can begin to see why the alternative to the mechanistic, scientific view of reality should so often be seen as the mentalistic, and indeed, hyper-mentalistic one enshrined in magic, superstition, and religion (Badcock 2004; in preparation).

\section{R5.6. Savantism and extraordinary skills}

Much the same point as made for religion in the preceding section might answer Nettle's question about why what we term mentalistic, rather than mechanistic, skills are attractive to women. Notwithstanding the ingenious suggestion he derives from our theory, it might simply be that mentalistic excellence is inherently more appealing to the more mentalistic sex. But however that may be, both Nettle's and Fitzgerald \& Hawi's comments raise an issue about creativity to which we would like to suggest a new kind of answer, based on our concept of a mentalistic continuum running from autism to psychosis. This is the suggestion that, if there are autistic savants - as there indeed are (Hermelin 2001; Treffert 2000) - then there also might be psychotic savants.

Autistic savantism is characterized by outstanding, if isolated, mechanistic skills or expertise set against a background of general mentalistic deficits. Accordingly, we might predict that psychotic savantism should show the exact opposite cognitive configuration: remarkable, if perhaps highly circumscribed, mentalistic talents coexisting with more general mechanistic deficits. By this term we do not mean to suggest that the savants in question are in fact psychotic, only that their cognitive configuration puts them on the psychotic side of the mentalistic 
spectrum. Nevertheless, the symmetry cannot be exact. For a start, the normally sad plight of autistics reminds us that mentalistic deficits are typically much more significant socially and have an enormous impact on people's personal relationships in a way in which mechanistic deficits seldom if ever do. Not being able to program the video, change a plug, or read a map is one thing, but failing to understand other people's motives, actions, and intentions is quite another - and much more damaging from a social point of view. Hyper-mentalistic tendencies of the kind seen in psychotic savants might normally promote a person's social adjustment because of the skill these consummate mentalists have in manipulating others and exploiting them thanks to their natural empathic understanding - particularly of other people's weaknesses. Consequently, psychotic savants are likely to be identified at worst as cranks or charlatans rather than psychopaths, and at best we should not expect psychotic savants to be as noticeable as autistic ones usually are, or as readily diagnosed as such.

Again, the areas of expertise involved in psychotic savantism might not be so obviously striking as calendarcalculation, photographic memory, or computer-like maths skills are. By contrast, hyper-mentalistic savantism might be expected in skills and areas of expertise that are much closer to normal social life and everyday concerns. Examples might be outstanding achievement in religious and political evangelism; literary and theatrical culture; litigation and the law; hypnosis, faith-healing, and psychotherapy; fashion, advertising, and public relations; and commerce, confidence-trickery, and fraud of all kinds.

Where literature is concerned, female poets have been as notable as female writers of detective fiction (see our reply to Thakkar et al. further on), thanks perhaps to the verbal, metaphorical, and abstract nature of the written word. Moreover, and as Fitzgerald \& Hawi hint in their general comment about bipolar illness, there is now good evidence that many of the world's leading poets of both sexes have shown signs of psychotic-spectrum disorders, most notably manic-depressive illnesses. A study of all major British and Irish poets born between 1705 and 1805 found a strikingly high rate of mood disorders, suicide, and institutionalization within this group of writers and their families (Jamison 1993), and Nettle (2006) reported that poets and artists have "levels of unusual experiences that are higher than controls, and as high as schizophrenia patients" (p. 876). As noted by Jamison (1993), "manic-depressive illness, unlike schizophrenia or Alzheimer's disease, is not a dementing illness. It may on occasion result in episodes of acute psychosis and flagrant irrationality, but these bouts of madness are almost always temporary and seldom progress to chronic insanity" (Jamison 1993, p. 96). Consequently, as in the perhaps parallel but mentalistically opposite case of Asperger's savants (Fitzgerald 2005), psychotic savants with a manic-depressive cognitive configuration may be sufficiently normal most of the time to be able to realize the advantages of their extended mentalism without being too disabled by it (e.g., Claridge et al. 1990). Indeed, even some features associated with the manic side of bipolar disorder, such as outgoingness, increased energy, intensified sexuality, increased risktaking, persuasiveness, self-confidence, and heightened productivity might contribute to success in many walks of life as long as they were not taken to extremes (Jamison 1993, p. 87). Even depressive episodes may contribute in view of the finding that mildly depressed people may be more realistic and objective than non-depressed ones (Taylor \& Brown 1988). This might explain why three quarters of studies report a link between manicdepressive illness and the professional or upper classes across several cultures and in different historical periods (Jamison 1993, p. 86). If autistic tendencies have contributed to mathematics, science, and technology, mentalistic ones have also clearly done so as far as literature, art, religion, and society are concerned.

Thakkar et al. also ask, "is there room for Sherlock Holmes in C\&B's spectrum?" Indeed there is, but an even more telling question might be whether there is also a place for Miss Marple. A number of authors have already noted Holmes's autistic traits and the critical role they play in his success as a detective (U. Frith 2003; Rennison 2005, p. 10). Indeed, Fitzgerald has described him as one of the most notable fictional portrayals of an Asperger's savant (Fitzgerald 2005, p. 85). However, it has been pointed out that Miss Marple, another of the immortals of detective fiction, "is in every way the opposite" of Holmes or Agatha Christie's other famous creation, Hercule Poirot, "and has no autistic traits at all: she solves crimes by intuition, immersing herself in the context without analytic deduction" (U. Frith 2003, p. 24). Could this be because Miss Marple is female, and therefore likely to be of a more mentalistic turn of mind, as described in our reply to Kanazawa? If so, then both mentalistic and hyper-mentalistic aspects can be found fused with autistic ones in what is perhaps the most distinctive literary genre of modern times: the detective story. This apparent pattern may explain why thrillers are so endlessly fascinating and why detective fiction has emerged as such a major factor in modern literature. Could it be that, rather than adopt a safe, central, normal mix of mentalistic and mechanistic cognition comparable to that found in most people, detective stories balance hyper-mentalistic paranoid suspicion against hypo-mentalistic autistic obsession, with conflicting detail to get the best of both? At the very least, this would explain why autistic characters like Sherlock Holmes and highly mentalistic ones like Miss Marple could both make convincing fictional detectives - and why so many female writers have made a success of their detective fiction (Badcock, in preparation).

\section{R6. Mentalistic and mechanistic cognition}

\section{R6.1. Sources of mentalistic deficits}

Langdon \& Brock touch on the issue of mentalistic deficits in psychosis and rightly point out that "it all depends on what one means by mentalizing." In reply, we might add that Troisi's comments on psychopathy and Williams syndrome highlight the contrast with Baron-Cohen's alternative concept of empathizing, which Troisi notes did not receive much attention in our original article but which Belmonte sees as extended and supplanted by our concept of mentalism. Baron-Cohen describes empathizing as "the drive to identify another person's emotions and thoughts, and 
to respond to these with an appropriate emotion" (Baron-Cohen 2005, p. 468), and he has suggested that deficits in empathizing are core features of autism (Baron-Cohen 2002; 2003). But as Troisi says, the fact that the empathic deficit in psychopathy is not associated with any other deficit in social cognition clearly contradicts the contention that such deficits critically characterize autism. On the contrary, as he observes, individuals with Williams syndrome show abnormally high levels of empathy combined with a defective theory of mind, again suggesting that empathizing deficits cannot be the central factor in autism. However, neither psychopathy nor Williams syndrome contradict our belief that in ASD, mentalism as defined here is underdeveloped, and that in psychosis, it is hypertrophied. According to our view, Troisi is correct in implying that psychopathy represents a normal or better-thannormal development of mentalism except for its empathizing element, which is pathologically absent, and that Williams syndrome represents the exact converse: spared empathizing ability but with notable deficits in other aspects of mentalism. In other words, our contention is that, while mentalism includes empathizing as an important element, the concept embraces much more and is closer to Baron-Cohen's earlier, modular model of the social mind (Badcock 2004; Baron-Cohen 1995).

Langdon \& Brock's comments highlight a further important aspect of our concept of mentalism. Another way of describing it would be as our evolved cognitive system for interacting with other human beings, by contrast to mechanistic cognition: the cognitive system which we evolved for interaction with the physical, nonhuman environment (Badcock 2004). This crucial distinction is highlighted in Langdon \& Brock's discussion of visualperspective taking and specifically in Langdon and Coltheart (2001). Here, we would argue that tasks involving purely visual perspective-taking should be seen as mechanistic in contrast to tasks involving mentalistic perspective-taking. Indeed, we would point out that the false-photo task illustrates the former by comparison with tests of false belief, which represent the latter, and that although autistics symptomatically do worse than normal on false belief, they often do as well or better than normal on the false-photo task (Leekam \& Perner 1991; Leslie \& Thaiss 1992; Zaitchik 1990). Furthermore, the fact that autistic artists often show remarkable natural perspective skills and are good at guessing how a scene will appear from another viewpoint, underlines the point that some of the skills assessed in the cases cited by Langdon \& Brock might not be mentalistic and consequently do not provide evidence against our view (Grandin \& Johnson 2005, p. 300). Alternatively, one could interpret their results as confirming the mentalistic/mechanistic distinction - not to mention that, just as our theory predicts, schizotypals are poor at mechanistic perspective skills (Langdon \& Coltheart 2001). As Belmonte perceptively comments, the mentalistic/mechanistic distinction applies not merely to the social brain, but also to cognition in general. Indeed, Badcock (in preparation) notes that sub-tests that show large sex differences are routinely excluded from standard measures of IQ (e.g., tests of mechanical comprehension or "folk physics"), and he suggests that their inclusion might indicate that there are two fundamental types of intelligence, rather than one. A distinction between conventional IQ and social or emotional intelligence has already been suggested (Marlowe 1986; Salovey \& Mayer 1990), but the fundamental distinction may be one of mentalistic as opposed to mechanistic intelligence. Recent studies showing superior fluid intelligence in autism (Dawson et al. 2007; Hayashi et al. 2008) accord well with this perspective, given that such cognitive abilities are abstract, mechanically inferential, and independent of acquired knowledge.

\section{R6.2. Hyper-mentalism}

Abu-Akel comments that "The target article discusses disorders affecting the social brain on 'a continuum of cognitive architecture from mechanistic to mentalistic cognition' [...] in which autism and schizophrenia are the extremes." However, we would add that it also discusses disorders affecting the social brain on a continuum of mentalism understood as our evolved capacity to interpret our own and others' behavior in purely mental terms (Badcock 2008). As such, mentalism goes beyond theory of mind to include empathy, emotion, self-awareness, and all other aspects of cognition that could be termed mental in any sense (Badcock, in preparation). We would strongly underline and endorse Abu-Akel's observation that both the hypo-mentalism of autism and the hyper-mentalism of psychosis can and often do result in mentalistic deficits - an important point that we have not made sufficiently clear for a number of commentators. Indeed, we never claimed that psychotics would necessarily demonstrate superior mentalistic skills across the board, so to speak, only that enhanced sensitivity or superior ability might be found in certain respects, such as sensitivity to direction of gaze (Langdon et al. 2006b), or to certain types of nonverbal cues (LaRusso 1978). The fact that symptoms like delusions of being watched or spied upon can be understood as pathological sensitivity to gaze, that delusions of being loved (erotomania) or hated (persecution) can be seen as hypertrophied interpretations of other people's intentions, or that delusions of conspiracies can be understood as shared attention run amok, does not imply that any of these instances of hyper-mentalism are necessarily real or accurate - on the contrary, the whole point is that they represent bizarre failures of normal mentalistic interpretation. Just as both under-sensitivity and over-sensitivity to light or sound can be expected to produce impairments in sight or hearing, so both over-sensitivity and under-sensitivity to mental factors can result in measurable mentalistic deficits. In the same way that a teacher who had a hyperactive child in the class would not be likely to find that child doing extra homework, so we should not assume that hyper-mentalism is simply normal mentalizing, plus some more. Quite the opposite, excessive mentalizing will result in outcomes that may at times look very like autistic deficits, such as avoidance of social contact; misunderstanding of other's intentions, emotions, or expressions; inability to appreciate humour or irony; misuse or underuse of mentalistic language; and so on.

As a specific example, autistics can often feel that people are against them and that they are being persecuted, just as some psychotics do, and such paranoia in both conditions could be interpreted as indicative of a common underlying cause (van Rijn et al.). However, the only laboratory study 
undertaken at the time of writing that compared Asperger's with paranoid-schizophrenic (and delusional-disorder) patients found that the low-level paranoid symptoms in the autistic group arise as a consequence of different mechanisms to those involved in psychotic delusions (Craig et al. 2004). Moreover, Blackshaw et al. (2001, p. 158) concluded that "the paranoia observed in Asperger syndrome ... does not appear to stem from the same factors as seen in the paranoia of people with a diagnosis of schizophrenia," and the authors suggest that "the paranoia seen in Asperger syndrome is of a different 'quality' to that observed in schizophrenia... [I]t may stem from a confusion of not understanding the subtleties of social interactions and social rules" rather than being seen as part of a "plot" against them (Blackshaw et al. 2001). Although a recent report found verbal - but no nonverbal - theory-of-mind deficits in schizophrenics with persecutory delusions (Harrington et al. 2005a), an earlier study which looked specifically at paranoia concluded that theory-of-mind deficits were not causal or specific to paranoid delusions. Instead, its authors suggested that such delusions may be secondary effects resulting from information-processing overload in schizophrenics (Drury et al. 1998). Earlier still, Bowler (1992) used schizophrenics as a control group when he examined theory-of-mind skills in Asperger syndrome and explicitly stated that the schizophrenics' social impairment "does not appear to result from an impaired theory of mind" (p. 888). Another study - that of Pickup (2006), cited by van Rijn et al. - found that although higher scores on a single dimension of schizotypy were associated with poorer theory-of-mind ability, no association was found between theory of mind and either total ratings of schizotypy or ratings of schizotypal traits analogous to the "behavioral signs" of schizophrenia (Pickup 2006). Furthermore, the same study pointed out that "it could be argued that poor scores on ToM tasks are related to general deficits in attention or motivation, lower IQ, higher doses of antipsychotic medication, poor memory, and/or greater severity of illness" (Pickup 2006, p. 178). Finally, a recent metaanalysis of 32 studies of theory of mind in schizophrenia which cited Abu-Akel's anticipation of our concept of hyper-mentalism concluded that a comparison of autistic and psychotic mentalism from this point of view might be a promising topic for future research (Sprong et al. 2007). At the very least, this establishes that a hyper-mentalistic interpretation of psychotic deficits in theory-of-mind skills is still an open question; and at best, that we may expect future research to explore the possibility in a manner not seen up to now.

\section{R6.3. Psychoanalysis}

Behrendt's commentary is neo-Freudian to the extent that he implicates "early life experiences" in schizophrenia, describes persecutory delusions as "defences," and charts Kleinian "positions" in his figure. A parallel between behaviorism and autistic hypo-mentalism has already been drawn (Baron-Cohen 1989), and the symmetry that we propose between autistic- and psychotic-spectrum disorders suggests another between psychoanalysis and hyper-mentalism. Whereas behaviorism denied the mind and banished all consideration of mental content as "unscientific," neo-Freudian psychoanalysis anathematized any recognition of the physical, biological, or genetic basis of the mind as "un-psychological." As a result, mentalistic cognition completely supervened over the more mechanistic core originally proposed by Freud himself in his instinct theory (Badcock 2002). Behrendt's commentary is clearly more balanced, but does prompt several observations regarding psychoanalysis.

Behaviorism could never have discovered the distinctive mentalistic deficits that characterize autism because it ruled the mind out of the question. But the implication of our theory is that psychoanalytic hypermentalism also disqualifies it from insight into psychotic-spectrum disorders for the contrary reason: the excessive mentalizing of neo-Freudian theory and practice makes them part of the problem, not a solution to it. With the benefit of hindsight, traditional psychoanalysis seems the very worst possible therapy that anyone on the psychotic side of the mentalistic spectrum could receive. By encouraging the patient to mentalize randomly - in other words, to free-associate, fantasize, and report their dreams - therapists would be encouraging the very factor that we argue is a root cause of psychotic symptoms: mentalizing to excess. At best, this could hardly help patients with hyper-mentalistic tendencies, and at worst it might be expected to do real harm. Indeed, the hyper-reflexive self-observation that Sass (2001) described as a major characteristic of schizophrenia was institutionalized in psychoanalytic therapy. Sass quotes a schizophrenic who remarked that "My downfall was insight. ... Too much insight can be very dangerous, because you can tear your mind apart." This patient did not mention psychoanalysis but seems to allude to it when he added, "Well, look at the word 'analysis.' That means to break apart. When it turns upon the self, the mind would rip apart" (Sass 2001).

Where disorders at the opposite pole of the mentalistic continuum are concerned, Freudian child-analysts failed to understand autism and how it could be treated. Thanks to their tendency to over-attribute mentalism to children and to assume an unconscious, repressed foundation for the mind, because of their belief in so-called infantile amnesia (Freud 1905/1953, pp. 174-76), Freudians of all kinds were in the main blind to the revelation that autism was a disorder featuring serious mentalistic deficits.

Some success can be obtained with autistic children by teaching them to mind-read: in other words, to learn explicit mentalistic skills (Howlin et al. 1999). If it is indeed true that psychotics are the opposite of this, and implicitly over-mentalize where autistics under-mentalize, it follows that it might be worth trying the opposite of psychoanalysis: to teach psychotics the contrary skills to those prescribed for autistics. There is evidence that even in normal individuals who have suffered bereavement, avoidance of mentalizing their loss reduced grief symptoms after 14 months, and most certainly did not increase them, as conventional Freudian wisdom would have suggested. Avoiding unpleasant thoughts and emotions might not be such a bad thing after all (Bonanno et al. 1995). If so, an obvious therapeutic experiment would be to try to induce psychotics to be hypo-reflective - or more autistic, if you like - and consciously to try to avoid reading too much into each and every thing. 
References/Crespi \& Badcock: Psychosis and autism as diametrical disorders of the social brain

\section{R7. Sex and imprinting}

Kanazawa draws attention to an important difference between the imprinted brain theory presented here and the extreme male brain theory of autism originally suggested by Asperger (1944), and recently fully elaborated by Baron-Cohen (2002; 2003). As Kanazawa rightly infers, and as Badcock implied in his original suggestion regarding paranoia as a mentalistic polar opposite of autism, it might seem that paranoid schizophrenia corresponds to an extreme female brain (Badcock 2004). However, in the target article we have been careful to point out that, according to the theory proposed here, psychoses-like paranoia represent not so much an extreme female as an extreme maternal brain, with autism correspondingly being a question not simply of an extreme male brain, but of an extreme paternal one. Furthermore, as our Figure 6 suggests, sex-based variation can be held orthogonal to imprinted-gene variation to give gradations between four, rather than two, general outcomes.

Keller requests the basis for our contention that the male brain should be similar to the paternal brain. The answer is simply that all fathers are male and that both aspects are associated, via development, physiology, and behavior, with relatively high demands on the mother. Thus, there is considerable evidence that sons impose higher costs in gestation and rearing than do daughters (reviewed in Rickard et al. 2007), and higher demands from paternally expressed imprinted genes are well established from theory and data, as described in our target article. A recent article also provides imaging evidence that the social-brain network of females engenders higher altruistic cooperativeness than that of males (Yamasue et al., in press). The partial alignments of the paternal brain and male brain, and the maternal brain with a female brain, should not be seen as conflation, but as key determinants of human neurocognitive architecture, such that jointly considering a male-female axis and a paternal-maternal axis (Fig. 6), can successfully explain the male bias to autism, the female bias to relatively mild psychotic-spectrum conditions, and the relative severities of autism in females and schizophrenia in males. We agree with Belmonte that bringing Aristotelian humours and biles up to date requires the positing of much more modern axes structuring development, cognition, and disease risk, which can be sought in the genetic underpinnings of interactions between the development of sexual dimorphism and the effects of imprinting. Here, digit ratios may provide useful insights given their high heritability and documented associations with both sex and conditions on the autistic and psychotic spectra (Voracek) but to progress much further, the pleiotropic genes and developmental networks underlying digit ratios must be discerned.

\section{R8. Conclusions}

Integrating theory and data in the synthetic analysis of neurodevelopmental disorders is a goal shared by perhaps all of the commentators, and ourselves. Like autism itself, the intellectual underpinnings of this task tend to lack central coherence, as well as models of how the world works that are as yet sufficiently robust to allow efficient, directed progress. We have described what we view as a simple, coherent model that makes novel, falsifiable predictions regarding neurodevelopmental pattern and process, from genes to behavior. We believe that the model has survived an intellectual gauntlet of expert commentators not just intact, but stronger due to their perspectives and insights. For these we extend our thanks, as well as our apologies to contributors whose work we cannot discuss in more detail.

As for the future, at the level of genes, Haig and Davies \& Isles urge caution in the interpretation of genetic data from complex theory and sketchy empirical knowledge to date. We agree, but also suggest that full elaboration of testable theory, even if speculative in parts, provides a paradigm to target data collection. Animal models are invaluable to elucidate mechanisms (Burne et al.), but our exposition helps define their limits: For example, conservation of imprinting from mouse to man is only partial (Glaser et al. 2006; Luedi et al. 2007), and the mentalistic abilities of rodents, or even chimps (Povinelli 2000), may differ from ours in kind as well as scope. In silico and neurological studies, with emphasis on perceptual and attentional processes affecting development, can more closely address mechanisms of human cognition (Belmonte, Vladusich), but these must ultimately be connected with psychological constructs. Future psychological analyses require, as Langdon \& Brock and Troisi suggest, direct comparisons between individuals on the autistic spectrum and psychotic spectrum engaging in the same ecologically valid tasks that probe mechanistic versus mentalistic brain functions. The idea of our brains being subject to genomic conflicts may rest uneasily with psychologists, psychiatrists, and neuroscientists whose tasks are challenging enough already, but this cannot be helped. Our brains are imprinted, our cognition is not unitary (Hamilton 1996, pp. 133-35), and our psyches are adapted to comprehend both people and things.

\section{References}

[The letters " $a$ " and “ $r$ " before author's initials stand for target article and response references, respectively.]

Abdolmaleky, H. M., Cheng, K. H., Faraone, S. V., Wilcox, M., Glatt, S. J., Gao, F., Smith, C. L., Shafa, R., Aeali, B., Carnevale, J., Pan, H., Papageorgis, P., Ponte, J. F., Sivaraman, V., Tsuang, M. T. \& Thiagalingam, S. (2006) Hypomethylation of MB-COMT promoter is a major risk factor for schizophrenia and bipolar disorder. Human Molecular Genetics $15(21): 3132-45$. [GV]

Abel, K. M. (2004) Foetal origins of schizophrenia: Testable hypotheses of genetic and environmental influences. British Journal of Psychiatry 184:383-85. $[\mathrm{aBC}]$

Abel, K. M. \& Allin, M. (2006) Placental programming leading to mental ill health: Fetal growth and schizophrenia. In: The placenta and neurodisability, ed. P. Baker \& C. Sibley, pp. 118-36. Cambridge University Press. [aBC]

Abell, F., Happé, F. \& Frith, U. (2000) Do triangles play tricks? Attribution of mental states to animated shapes in normal and abnormal development. Journal of Cognitive Development 15:1-20. [RL]

Abramson, R. K., Ravan, S. A., Wright, H. H., Wieduwilt, K., Wolpert, C. M., Donnelly, S. A., Pericak-Vance, M. A. \& Cuccaro, M. L. (2005) The relationship between restrictive and repetitive behaviors in individuals with autism and obsessive compulsive symptoms in parents. Child Psychiatry and Human Development 36:155-65. [aBC]

Abu-Akel, A. (1999) Impaired theory of mind in schizophrenia. Pragmatics and Cognition 7:247-82. [aBC]

(2003) A neurobiological mapping of theory of mind. Brain Research Review 43:29-40. [AA-A] 
Abu-Akel, A. \& Abushua'leh, K. (2004) “Theory of mind" in violent and nonviolent patients with paranoid schizophrenia. Schizophrenia Research 69:45-53. [aBC, AT]

Abu-Akel, A. \& Bailey, A. L. (2000) The possibility of different forms of theory of mind impairment in psychiatric and developmental disorders [Letter to the editor]. Psychological Medicine 30:735-38. [AA-A, aBC]

Adolphs, R., Baron-Cohen, S. \& Tranel, D. (2002) Impaired recognition of social emotions following amygdala damage. Journal of Cognitive Neuroscience $14: 1264-74 . \quad[\mathrm{aBC}]$

Adolphs, R., Damasio, H., Tranel, D., Cooper, G. \& Damasio, A. R. (2000) A role for somatosensory cortices in the visual recognition of emotion as revealed by three-dimensional lesion mapping. Journal of Neuroscience 20:2683-90. [R-PB]

Adolphs, R., Gosselin, F., Buchanan, T. W., Tranel, D., Schyns, P. \& Damasio, A. R. (2005) A mechanism for impaired fear recognition after amygdala damage. Nature 433:68-72. [aBC]

Akefeldt, A., Ekman, R., Gillberg, C. \& Mansson, J. E. (1998) Cerebrospinal fluid monoamines in Prader-Willi syndrome. Biological Psychiatry 44:1321-28. $[\mathrm{aBC}]$

Akhondzadeh, S., Rezaei, F., Larijani, B., Nejatisafa, A. A., Kashani, L. \& Abbasi, S. H. (2006) Correlation between testosterone, gonadotropins and prolactin and severity of negative symptoms in male patients with chronic schizophrenia. Schizophrenia Research 84:405-10. [aBC]

Alaghband-Rad, J., McKenna, K. \& Gordon, C. T. (1995) Childhood onset schizophrenia: The severity of premorbid course. Journal of the American Academy of Child and Adolescent Psychiatry 34:1275-83. [ST]

Alberti, A., Romano, C., Falco, M., Calì, F., Schinocca, P., Galesi, O., Spalletta, A., Di Benedetto, D. \& Fichera, M. (2007) 1.5 Mb de novo 22q11.21 microduplication in a patient with cognitive deficits and dysmorphic facial features. Clinical Genetics 71:177-82. [rBC]

Aleman, A., Bocker, K. B., Hijman, R., de Haan, E. H. \& Kahn, R. S. (2003) Cognitive basis of hallucinations in schizophrenia: Role of top-down information processing. Schizophrenia Research 64(2-3):175-85. [TV]

Aleman, A. \& Kahn, R. S. (2005) Strange feelings: Do amygdala abnormalities dysregulate the emotional brain in schizophrenia? Progress in Neurobiology $77: 283-98 . \quad[\mathrm{aBC}]$

Aleman, A., Kahn, R. S. \& Selten, J. P. (2003) Sex differences in risk for schizophrenia: Evidence from meta-analysis. Archives of General Psychiatry 60:565-71. [aBC]

Alexander, A. L., Lee, J. E., Lazar, M., Boudos, R., Dubray, M. B., Oakes, T. R., Miller, J. N., Lu, J., Jeong, E., McMahon, W. M., Bigler, E. D. \& Lainhart, J. E. (2007) Diffusion tensor imaging of the corpus callosum in autism. NeuroImage 34:61-73. [aBC]

Alexander, R. D. (1979) Darwinism and human affairs. Pitman. [aBC] (1987) The biology of moral systems. Aldine De Gruyter. [aBC]

(1989) Evolution of the human psyche. In: The human revolution: Behavioural and biological perspectives on the origins of modern humans, ed. P. Mellars \& C. Stringer, pp. 455-513. Edinburgh University Press. [aBC]

Allen, N. D., Logan, K., Lally, G., Drage, D. J., Norris, M. L. \& Keverne, E. B. (1995) Distribution of parthenogenetic cells in the mouse brain and their influence on brain development and behavior. Proceedings of the National Academy of Sciences USA 92:10782-86. [BJAD, LMG]

Anderson, G. M., Jacobs-Stannard, A., Chawarska, K., Volkmar, F. R. \& Kliman, H J. (2007) Placental trophoblast inclusions in autism spectrum disorder. Biological Psychiatry 61:487-91. [aBC]

Andersson, M. B. (1994) Sexual selection. Princeton University Press. [aBC]

Andrews, S. C., Wood, M. D., Tunster, S. J., Barton, S. C., Surani, M. A. \& John, R. M. (2007) Cdknlc (p57Kip2) is the major regulator of embryonic growth within its imprinted domain on mouse distal chromosome 7. BMC Developmental Biology 7:53. [rBC]

Angiolini, E., Fowden, A., Coan, P., Sandovici, I., Smith, P., Dean, W., Burton, G., Tycko, B., Reik, W., Sibley, C. \& Constância, M. (2006) Regulation of placental efficiency for nutrient transport by imprinted genes. Placenta 27(A):98-102. [aBC]

Antshel, K. M., Aneja, A., Strunge, L., Peebles, J., Fremont, W. P., Stallone, K., Abdulsabur, N., Higgins, A. M., Shprintzen, R. J. \& Kates, W. R. (2007) Autistic spectrum disorders in velo-cardio facial syndrome (22q11.2 deletion). Journal of Autism and Developmental Disorders 37:1776-86. [aBC]

Arató, M., Frecska, E., Beck, C., An, M. \& Kiss, H. (2004) Digit length pattern in schizophrenia suggests disturbed prenatal hemispheric lateralization. Progress in Neuro-Psychopharmacology and Biological Psychiatry 28:191-94. [aBC, MV]

Arbelle, S., Sigman, M. D. \& Kasari, C. (1994) Compliance with parental prohibition in autistic children. Journal of Autism and Developmental Disorders 24:693-702. [aBC]

Arbib, M. A. \& Mundhenk, T. N. (2005) Schizophrenia and the mirror system: An essay. Neuropsychologia 43:268-80. [aBC]

Arguello, P. A. \& Gogos, J. A. (2006) Modeling madness in mice: One piece at a time. Neuron 52(1):179-96. [THJB]
Arking, D. E., Cutler, D. J., Brune, C. W., Teslovich, T. M., West, K., Ikeda, M., Rea, A., Guy, M., Lin, S., Cook, E. H. \& Chakravarti, A. (2008) A common genetic variant in the neurexin superfamily member CNTNAP2 increases familial risk of autism. American Journal of Human Genetics 82:160-64. $[\mathrm{rBC}]$

Asarnow, J. R., Tompson, M. C. \& Goldstein, M. J. (1994) Childhood-onset schizophrenia: A follow-up study. Schizophrenia Bulletin 20(4):599-617. [ST]

Asperger, H. (1944) Die "autistichen psychopathen" im kindesalter [Autistic psychopathy in infancy]. Archiv für Psychiatrie und Nervenkrankheiten [Archive for Psychiatry and Nerve Disease] 117:76-136. [rBC]

(1991) Autistic psychopathy in childhood. In: Autism and asperger syndrome, ed. U. Frith. Cambridge University Press. [aBC]

Atlas, J. A. \& Lapidus, L. B. (1987) Patterns of symbolic expression in subgroups of the childhood psychoses. Journal of Clinical Psychology 43:177-88. $[\mathrm{aBC}]$

Atran, S. (2002) In gods we trust: The evolutionary landscape of religion. Oxford University Press. [SK]

Avila, M., Thaker, G. \& Adami, H. (2001) Genetic epidemiology and schizophrenia: A study of reproductive fitness. Schizophrenia Research 47:233-41. [aBC, MCK]

Aylward, E. H., Minshew, N. J., Goldstein, G., Honeycutt, N. A., Augustine, A. M., Yates, K. O., Barta, P. E. \& Pearlson, G. D. (1999) MRI volumes of amygdala and hippocampus in non-mentally retarded autistic adolescents and adults. Neurology 53:2145-50. [MKB, R-PB]

Bachmann, S., Pantel, J., Flender, A., Bottmer, C., Essig, M. \& Schroder, J. (2003) Corpus callosum in first-episode patients with schizophrenia - A magnetic resonance imaging study. Psychological Medicine 33:1019-27. [aBC]

Badcock, C. R. (2000) Evolutionary psychology: A critical introduction. Polity Press. $[\mathrm{aBC}]$

(2002) Incest, the libido theory, and sociobiology and psychoanalysis. In: The Freud encyclopedia, ed. E. Erwin, pp. 273-75, 321-24, 534-35. Routledge. $[\mathrm{rBC}]$

(2004) Mentalism and mechanism: The twin modes of human cognition. In: Human nature and social values: Implications of evolutionary psychology for public policy, ed. C. Crawford \& C. Salmon, pp. 99-116. Erlbaum. (Published online in 2002.) [arBC]

(2008) An evolutionary theory of mind and of mental illness: Genetic conflict and the mentalistic continuum. In: Foundations of evolutionary psychology: Ideas, issues and applications, ed. C. Crawford \& D. Krebs, pp. 430-50. Erlbaum. $[\mathrm{rBC}]$

(in preparation) Autism to mind cancer: A new view of the mind and mental illness. $\quad[\mathrm{rBC}]$

Badcock, C. \& Crespi, B. (2006) Imbalanced genomic imprinting in brain development: An evolutionary basis for the aetiology of autism. Journal of Evolutionary Biology 19:1007-32. [aBC]

Badner, J. A. \& Gershon, E. S. (2002) Regional meta-analysis of published data supports linkage of autism with markers on chromosome 7. Molecular Psychiatry 7(1):56-66. [MCK]

Baethge, C., Baldessarini, R. J., Freudenthal, K., Streeruwitz, A., Bauer, M. \& Bschor, T. (2005) Hallucinations in bipolar disorder: Characteristics and comparison to unipolar depression and schizophrenia. Bipolar Disorders $7: 136-45 . \quad[\mathrm{aBC}]$

Bailer, J., Brauer, W. \& Rey, E. R. (1996) Premorbid adjustment as predictor of outcome in schizophrenia: Results of a prospective study. Acta Psychiatrica Scandinavica 93(55):368-77. [ST]

Bailey, A. A. \& Hurd, P. L. (2005) Depression in men is associated with more feminine finger length ratios. Personality and Individual Differences 39: 829-36. $[\mathrm{aBC}]$

Barak, Y., Achiron, A., Mandel, M., Mirecki, I. \& Aizenberg, D. (2005) Reduced cancer incidence among patients with schizophrenia. Cancer 104:2817-21. $[\mathrm{aBC}]$

Barnett, K. J. \& Corballis, M. C. (2002) Ambidexterity and magical ideation. Laterality 7:75-84. $\quad[\mathrm{aBC}]$

Barnett, K. J., Corballis, M. C. \& Kirk, I. J. (2005) Symmetry of callosal information transfer in schizophrenia: A preliminary study. Schizophrenia Research 74:171-78. [aBC]

Barnett, K. J. \& Kirk, I. J. (2005) Lack of asymmetrical transfer for linguistic stimuli in schizophrenia: An ERP study. Clinical Neurophysiology 116:1019-27. [aBC]

Baron, C. A., Tepper, C. G., Liu, S. Y., Davis, R. R., Wang, N. J., Schanen, N. C. \& Gregg, J. P. (2006) Genomic and functional profiling of duplicated chromosome 15 cell lines reveal regulatory alterations in UBE3A-associated ubiquitin-proteasome pathway processes. Human Molecular Genetics $15: 853-69 . \quad$ [rBC]

Baron-Cohen, S. (1989) Are autistic children "behaviorists"? An examination of their mental-physical and appearance-reality distinctions. Journal of Autism and Developmental Disorders 19:579-600. [rBC] 
(1995) Mindblindness: An essay on autism and theory of mind. MIT Press. [arBC, KNT]

(2002) The extreme male brain theory of autism. Trends in Cognitive Sciences 6:248-54. [MKB, arBC]

(2003) The essential difference: The truth about the male and female brain. Basic Books/Penguin. [arBC, SK]

(2005) The empathizing system: A revision of the 1994 model of the mindreading system. In: Origins of the social mind, ed. B. J. Ellis \& D. F. Bjorklund, pp. 468-92. Guilford Press. [rBC]

(2006) Two new theories of autism: Hyper-systemising and assortative mating. Archives of Disease in Childhood 91(1):2-5. [GV]

Baron-Cohen, S. \& Belmonte, M. K. (2005) Autism: A window onto the development of the social and the analytic brain. Annual Review of Neuroscience 28:109-26. [aBC]

Baron-Cohen, S., Bolton, P., Wheelwright, S., Scahill, V., Short, L., Mead, G. \& Smith, A. (1998) Autism occurs more often in families of physicists, engineers and mathematicians. Autism 2:296-301. [DN, SK]

Baron-Cohen, S., Knickmeyer, R. C. \& Belmonte, M. K. (2005) Sex differences in the brain: Implications for explaining autism. Science 310:819-23. [aBC]

Baron-Cohen, S., Ring, H. A., Bullmore, E. T., Wheelwright, S., Ashwin, C. \& Williams, S. C. (2000) The amygdala theory of autism. Neuroscience and Biobehavioral Reviews 24:355-64. [aBC]

Baron-Cohen, S., Wheelwright, S., Skinner, R., Martin, J. \& Clubley, E. (2001) The autism-spectrum quotient (AQ): Evidence from Asperger syndrome/high-functioning autism, males and females, scientists and mathematicians. Journal of Autism and Developmental Disorders 31:5-17. [aBC]

Barrantes-Vidal, N. (2004) Creativity and madness revisited from current psychological perspectives. Journal of Consciousness Studies 11:58-78. [aBC]

Barry, R. J., Leitner, R. P., Clarke, A. R. \& Einfeld, S. L. (2005) Behavioral aspects of Angelman syndrome: A case control study. American Journal of Medical Genetics: Part A 132:8-12. [rBC]

Bassett, A. S., Bury, A., Hodgkinson, K. A. \& Honer, W. G. (1996) Reproductive fitness in familial schizophrenia. Schizophrenia Research 21:151-60. [arBC, MCK]

Baum, K. M. \& Walker, E. F. (1995) Childhood behavioral precursors of adult symptom dimensions in schizophrenia. Schizophrenia Research 16:111-20. [ST]

Baumhackl, M. (2007) Das Fingerlängenverhältnis (2D:4D) der rechten und linken Hand als Maß der pränatalen Testosteronkonzentration und zerebrale Aktivierungsasymmetrien bei schizophrenen und affektiven Psychosen [Righthand and left-hand finger-length ratio (2D:4D) as a marker of prenatal testosterone levels and cerebral activational asymmetries in schizophrenic and affective psychoses]. Unpublished Masters thesis, University of Graz, Austria. [MV]

Bayliss, A. P., di Pellegrino, G. \& Tipper, S. P. (2005) Sex differences in eye gaze and symbolic cueing of attention. Quarterly Journal of Experimental Psychology 58:631-50. [aBC]

Bearden, C. E., Woodin, M. F., Wang, P. P., Moss, E., McDonald-McGinn, D., Zackai, E., Emannuel, B. \& Cannon, T. D. (2001) The neurocognitive phenotype of the 22q11.2 deletion syndrome: Selective deficit in visual-spatial memory. Journal of Clinical and Experimental Neuropsychology 23:447-64. $[\mathrm{rBC}]$

Behrendt, R. P. (1998) Underconstrained perception: A theoretical approach to the nature and function of verbal hallucinations. Comprehensive Psychiatry 39:236-48. [R-PB]

(2004) A neuroanatomical model of passivity phenomena. Consciousness and Cognition 13:579-609. [aBC]

(2005) Passivity phenomena: Implications for the concept of self. NeuroPsychoanalysis 7:185-207. [R-PB]

(2006a) Cruelty as by-product of ritualisation of intraspecific aggression in cultural evolution. (Commentary) Behavioral and Brain Sciences 29:226-27. [R-PB]

(2006b) Dysregulation of thalamic sensory transmission in schizophrenia: Neurochemical vulnerability to hallucinations. Journal of Psychopharmacology 20:356-72. [R-PB]

(2006c) The desire to obtain money: A culturally ritualised expression of the aggressive instinct. (Commentary) Behavioral and Brain Sciences 29:178-79. [R-PB]

(2006d) The social environment compresses the diversity of genetic aberrations into the uniformity of schizophrenia manifestations. (Commentary) Behavioral and Brain Sciences 29:406-408. [R-PB]

(2006e) The subjectivity of the perceived world: Psychopathology and the mind-body problem from a perspective of idealism. In: New developments in consciousness research, ed. V. W. Fallio, pp. 189-209. Nova Science. [R-PB]

Bejerot, S. (2007) An autistic dimension: A proposed subtype of obsessive-compulsive disorder. Autism 11:101-10. [aBC]
Bejerot, S. \& Nylander, L. (2003) Low prevalence of smoking in patients with autism spectrum disorders. Psychiatry Research 119:177-82. [aBC]

Bellgrove, M. A., Vance, A. \& Bradshaw, J. L. (2003) Local-global processing in early-onset schizophrenia: Evidence for an impairment in shifting the spatial scale of attention. Brain and Cognition 51:48-65. [aBC]

Belmonte, M. K., Allen, G., Beckel-Mitchener, A., Boulanger, L. M., Carper, R. A \& Webb, S. J. (2004a) Autism and abnormal development of brain connectivity. Journal of Neuroscience 24:9228-31. [aBC]

Belmonte, M. K. \& Bourgeron, T. (2006) Fragile X syndrome and autism at the intersection of genetic and neural networks. Nature Neuroscience 9:1221-25. [aBC]

Belmonte, M. K. \& Carper, R. A. (2006) Monozygotic twins with Asperger syndrome: Differences in behaviour reflect variations in brain structure and function. Brain and Cognition 61(1):110-21. [LMG]

Belmonte, M. K., Cook, E. H., Jr., Anderson, G. M., Rubenstein, J. L., Greenough, W. T., Beckel-Mitchener, A., Courchesne, E., Boulanger, L. M., Powell, S. B., Levitt, P. R., Perry, E. K., Jiang, Y. H., DeLorey, T. M. \& Tierney, E. (2004b) Autism as a disorder of neural information processing: Directions for research and targets for therapy. Molecular Psychiatry 9:646-63. [aBC]

Bender, L. \& Faetra, G. (1972) The relationship between childhood and adult schizophrenia. In: Genetic factors in schizophrenia, ed. A. R. Kaplan, pp. 28-64. Charles C. Thomas. [ST]

Benes, F. M. \& Berretta, S. (2001) GABAergic interneurons: Implications for understanding schizophrenia and bipolar disorder. Neuropsychopharmacology 25:1-27. [aBC]

Ben Shalom, D. (2000) Developmental depersonalization: The prefrontal cortex and self-functions in autism. Consciousness and Cognition 9:457-60. [aBC]

Bentall, R. P. (2003a) Madness explained: Psychosis and human nature. Allen Lane. [arBC, BJAD]

(2003b) The paranoid self. In: The self in neuroscience and psychiatry, ed. T. Kircher \& A. David, pp. 293-318. Cambridge University Press. [aBC]

Bentall, R. P. \& Kinderman, P. (1998) Psychological processes and delusional beliefs: Implications for the treatment of paranoid states. In: Outcome and innovation in psychological treatment of schizophrenia, ed. S. Lewis, N. Tarrier \& T. Wykcs, pp. 119-44. Wiley. [SvR]

Berg, J. S., Brunetti-Pierri, N., Peters, S. U., Kang, S. L., Fong, C., Salamone, J., Freedenberg, D., Hannig, V. L., Prock, L. A., Miller, D. T., Raffalli, P., Harris, D. J., Erickson, R. P., Cunniff, C., Clark, G. D., Blazo, M. A., Peiffer, D. A., Gunderson, K. L., Sahoo, T., Patel, A., Lupski, J. R., Beaudet, A. L. \& Cheung, S. W. (2007) Speech delay and autism spectrum behaviors are frequently associated with duplication of the $7 q 11.23$ Williams-Beuren syndrome region. Genetics in Medicine 9:427-41. [rBC]

Bering, J. M. (2002) The existential theory of mind. Review of General Psychology $6: 3-24 . \quad[\mathrm{rBC}]$

Berretta, S., Munno, D. W. \& Benes, F. M. (2001) Amygdalar activation alters the hippocampal GABA system: "Partial" modelling for postmortem changes in schizophrenia. Journal of Comparative Neurology 431:129-38. [aBC]

Bersani, G., Maneschi, M. L., Tarolla, E. \& Pancheri, P. (2006) Dyslexia as a possible aspect of neurocognitive impairment in schizophrenia. Schizophrenia Research 82:265-66. [aBC]

Bersani, G., Russo, D., Limpido, L. \& Marconi, D. (2007) Mood disorder in a patient with Smith-Magenis syndrome: A case report. Neuroendocrinology Letters 28:7-10. [rBC]

Bertella, L., Girelli, L., Grugni, G., Marchi, S., Molinari, E. \& Semenza, C. (2005) Mathematical skills in Prader-Willi Syndrome. Journal of Intellectual Disability Research 49:159-69. [aBC]

Bertone, A., Mottron, L., Jelenic, P. \& Faubert, J. (2005) Enhanced and diminished visuo-spatial information processing in autism depends on stimulus complexity. Brain 128:2430-41. [aBC]

Bigler, E. D., Mortensen, S., Neeley, E. S., Ozonoff, S., Krasny, L., Johnson, M., Lu, J., Provencal, S. L., McMahon, W. \& Lainhart, J. E. (2007) Superior temporal gyrus, language function, and autism. Developmental Neuropsychology 31:217-38. [aBC]

Birchwood, M., Gilbert, P., Gilbert, J., Trower, P., Meaden, A., Hay, I., Murray, E. \& Miles, J. N. (2004) Interpersonal and role-related schema influence the relationship with the dominant "voice" in schizophrenia: A comparison of three models. Psychological Medicine 34:1571-80. [aBC]

Bittel, D. C. \& Butler, M. G. (2005) Prader-Willi syndrome: Clinical genetics, cytogenetics and molecular biology. Expert Reviews in Molecular Medicine $7: 1-20 . \quad[\mathrm{aBC}, \mathrm{BJAD}]$

Bittel, D. C., Kibiryeva, N., Talebizadeh, Z. \& Butler, M. G. (2003) Microarray analysis of gene/transcript expression in Prader-Willi syndrome: Deletion versus UPD. Journal of Medical Genetics 40:568-74. [aBC]

Blackshaw, A. J., Kinderman, P., Hare, D. J. \& Hatton, C. (2001) Theory of mind, causal attribution and paranoia in Asperger syndrome. Autism 5(2):147-63. [rBC, SvR]

Blackwood, D. H. R., Pickard, B. J., Thomson, P. A., Evans, K. L., Porteous, D. J. \& Muir, W. J. (2007) Are some genetic risk factors common to schizophrenia, 
bipolar disorder and depression? Evidence from DISCl, GRIK4 and NRG1. Neurotoxicity Research 11:73-83. [aBC]

Blackwood, N. J., Howard, R. J., Bentall, R. P. \& Murray, R. M. (2001) Cognitive neuropsychiatric models of persecutory delusions. American Journal of Psychiatry 158:527-39. [aBC]

Blair, R. J. R. (2003) Neurobiological basis of psychopathy. British Journal of Psychiatry 182:5-7. [AT]

(2005) Responding to the emotions of others: Dissociating forms of empathy through the study of typical and psychiatric populations. Consciousness and Cognition 14:698-718. [aBC, RL]

Blanc, R., Adrien, J. L., Roux, S. \& Barthélémy, C. (2005) Dysregulation of pretend play and communication development in children with autism. Autism 9:229-45. [aBC]

Blanton, R. E., Levitt, J. G., Peterson, J. R., Fadale, D., Sporty, M. L., Lee, M., To, D., Mormino, E. C., Thompson, P. M., McCracken, J. T. \& Toga, A. W. (2004) Gender differences in the left inferior frontal gyrus in normal children. NeuroImage 22:626-36. [aBC]

Bleuler, E. (1911) Dementia Praecox oder Gruppe der Schizophrenien [Dementia praecox or group of schizophrenias]. Deutike. [SvR] Also in: Handbuch der Psychiatrie, ed. E. Bleuler \& P. Dover. Aschaffenburg. (1951 English edition, Textbook of Psychiatry, trans. H. A. Brill.) [ST]

Boer, H., Holland, A., Whittington, J., Butler, J., Webb, T. \& Clarke, D. (2002) Psychotic illness in people with Prader-Willi syndrome due to chromosome 15 maternal uniparental disomy. Lancet 359(9301):135-36. [WD]

Boks, M. P., de Vette, M. H., Sommer, I. E., van Rijn, S., Giltay, J. C., Swaab, H. \& Kahn, R. S. (2007a) Psychiatric morbidity and X-chromosomal origin in a Klinefelter sample. Schizophrenia Research 93:399-402. [arBC]

Boks, M. P., Leask, S., Vermunt, J. K. \& Kahn, R. S. (2007b) The structure of psychosis revisited: The role of mood symptoms. Schizophrenia Research 93:178-85. [aBC]

Bölte, S. \& Poustka, F. (2006) The broader cognitive phenotype of autism in parents: How specific is the tendency for local processing and executive dysfunction? Journal of Child Psychology and Psychiatry, and Allied Disciplines 47:639-45. [aBC]

Bonaglia, M. C., Giorda, R., Mani, E., Aceti, G., Anderlid, B., Baroncini, A., Pramparo, T. \& Zuffardi, O. (2006) Identification of a recurrent breakpoint within the SHANK3 gene in the $22 \mathrm{q} 13.3$ deletion syndrome. Journal of Medical Genetics 43:822-28. [rBC]

Bonanno, G. A., Holen, A., Keltner, D. \& Horowitz, M. J. (1995) When avoiding unpleasant emotions might not be such a bad thing: Verbal-autonomic response dissociation and midlife conjugal bereavement. Journal of Personality and Social Psychology 69:975-89. [rBC]

Boucher, J. (2007) Memory and generativity in very high functioning autism: A firsthand account, and an interpretation. Autism 11:255-64. [aBC]

Bowler, D. M. (1992) "Theory of mind" in Asperger's syndrome. Journal of Child Psychology and Psychiatry, and Allied Disciplines 33:877-93. [AA-A, arBC]

Boyer, P. (2001) Religion explained: The evolutionary origins of religious thought. Basic Books. [SK]

Bradshaw, J. L. \& Nettleton, N. C. (1983) Human cerebral asymmetry. Prentice Hall. $[\mathrm{aBC}]$

Brake, W. G., Sullivan, R. M. \& Gratton, A. (2000) Perinatal distress leads to lateralized medial prefrontal cortical dopamine hypofunction in adult rats. Journal of Neuroscience 20:5538-43. [aBC]

Brambilla, P., Cerini, R., Gasparini, A., Versace, A., Andreone, N., Vittorini, E. Barbui, C., Pelizza, L., Nosè, M., Barlocco, L., Marrella, G., Gregis, M., Tournikioti, K., David, A. S., Keshavan, M. S. \& Tansella, M. (2005) Investigation of corpus callosum in schizophrenia with diffusion imaging. Schizophrenia Research 79:201-10. [aBC]

Brambilla, P., Hardan, A. Y., di Nemi, S. U., Caverzasi, E., Soares, J. C., Perez, J. \& Barale, F. (2004) The functional neuroanatomy of autism. Functional Neurology 19:9-17. [aBC]

Brock, J., Brown, C., Boucher, J. \& Rippon, G. (2002) The temporal binding deficit hypothesis of autism. Development and Psychopathology 142(2):209-24. [CKD]

Broks, P. (1997) Brain, self, and others: The neuropsychology of social cognition. In: Schizotypy: Implications for illness and health, ed. G. Claridge, pp. 98-123. Oxford University Press. [aBC]

Brothers, L. (1990) The social brain: A project for integrating primate behavior and neurophysiology in a new domain. Concepts in Neuroscience 1:27-51. [aBC]

Brown, A. S. (2006) Prenatal infection as a risk factor for schizophrenia. Schizophrenia Bulletin 32(2):200-202. [THJB]

Brown, W. M. (2001) Genomic imprinting and the cognitive architecture mediating human culture. Journal of Cognition and Culture 1:251-58. [aBC]

Brown, W. M. \& Consedine, N. S. (2004) Just how happy is the happy puppet? An emotion signaling and kinship theory perspective on the behavioral phenotype of children with Angelman syndrome. Medical Hypotheses 63(3):377-85. [BJAD, WD]
Browne, K. R. (2005) Women in science: Biological factors should not be ignored. Cardozo's Women's Law Journal 11:509-28. [aBC]

Brugger, P. (2001) From haunted brain to haunted science: A cognitive neuroscience view of paranormal and pseudoscientific thought. In: Hauntings and poltergeists: Multidisciplinary perspectives, ed. J. Houran \& R. Lange, pp. 195-213. McFarland. [aBC]

Brugger, P. \& Graves, R. E. (1997a) Right hemispatial inattention and magical ideation. European Archives of Psychiatry and Clinical Neuroscience 247:55-57. [aBC]

(1997b) Testing vs. believing hypothesis: Magical ideation in the judgement of contingencies. Cognitive Neuropsychiatry 2:251-72. [aBC]

Brune, C. W., Kim, S. J., Salt, J., Leventhal, B. L., Lord, C. \& Cook, E. H. (2006) 5-HTTLPR genotype-specific phenotype in children and adolescents with autism. American Journal of Psychiatry 163:2148-56. [ST]

Brüne, M. (2004) Schizophrenia-an evolutionary enigma? Neuroscience and Biobehavioral Reviews 28:41-53. [aBC]

(2005) "Theory of mind" in schizophrenia: A review of the literature. Schizophrenia Bulletin 31(1):21-42. [KNT]

Brüne, M. \& Brüne-Cohrs, U. (2006) Theory of mind - Evolution, ontogeny, brain mechanisms and psychopathology. Neuroscience and Biobehavioral Reviews $30: 437-55 . \quad$ [aBC]

Brüne, M., Ribbert, H. \& Schiefenhövel, W. (2003) The social brain: Evolution and pathology. Wiley. [AT]

Brunet, A., Armengol, L., Pelaez, T., Guillamat, R., Vallès, V., Gabau, E., Estivill, X., \& Guitart, M. (2008) Failure to detect the 22q11.2 duplication syndrome rearrangement among patients with schizophrenia. Behavioral and Brain Functions 4:10. [rBC]

Brunet, A., Datta, S. R. \& Greenberg, M. E. (2001) Transcription-dependent and -independent control of neuronal survival by the PI3K-Akt signaling pathway. Current Opinion in Neurobiology 11:297-305. [aBC]

Brunet-Gouet, E. \& Decety, J. (2006) Social brain dysfunctions in schizophrenia: A review of neuroimaging studies. Psychiatry Research 148:75-92. [aBC]

Buck, C., Hobbs, G. E., Simpson, H. \& Wanklin, J. M. (1975) Fertility of the sibs of schizophrenic patients. British Journal of Psychiatry 127:235-39. [aBC]

Burd, L. \& Kerbeshian, J. (1988) Familial pervasive development disorder, Tourette disorder and hyperlexia. Neuroscience and Biobehavioral Reviews $12: 233-34 . \quad[\mathrm{aBC}]$

Burgess, N., Maguire, E. A. \& O’Keefe, J. (2002) The human hippocampus and spatial and episodic memory. Neuron 35:625-41. [aBC]

Bürgy, M. (2007) Obsession in the strict sense: A helpful psychopathological phenomenon in the differential diagnosis between obsessive-compulsive disorder and schizophrenia. Psychopathology 40:102-10. [aBC]

Burns, J. K. (2004) An evolutionary theory of schizophrenia: Cortical connectivity, metarepresentation, and the social brain. Behavioral and Brain Sciences 27:831-85. [aBC]

(2006a) Psychosis: A costly by-product of social brain evolution in Homo sapiens. Progress in Neuro-Psychopharmacology and Biological Psychiatry 30:797-814. [aBC]

(2006b) The social brain hypothesis of schizophrenia. World Psychiatry 5:77-81. [AT]

Bursch, B., Ingman, K., Vitti, L., Hyman, P. \& Zeltzer, L. K. (2004) Chronic pain in individuals with previously undiagnosed autistic spectrum disorders. Journal of Pain 5:290-95. [aBC]

Burt, A. \& Trivers, R. (2006) Genes in conflict: The biology of selfish genetic elements. Harvard University Press. [aBC]

Butler, M. G., Dasouki, M. J., Zhou, X. P., Talebizadeh, Z., Brown, M., Takahashi, T. N., Miles, J. H., Wang, C. H., Stratton, R., Pilarski, R. \& Eng, C. (2005) Subset of individuals with autism spectrum disorders and extreme macrocephaly associated with germline PTEN tumour suppressor gene mutations. Journal of Medical Genetics 42:318-21. [arBC, CKD]

Butler, P. D. \& Javitt, D. C. (2005) Early-stage visual processing deficits in schizophrenia. Current Opinion in Psychiatry 18:151-57. [MKB]

Butler, P. D., Martinez, A., Foxe, J. J., Kim, D., Zemon, V., Silipo, G., Mahoney, J., Shpaner, M., Jalbrzikowski, M. \& Javitt, D. C. (2007) Subcortical visual dysfunction in schizophrenia drives secondary cortical impairments. Brain $130: 417-30 . \quad$ [MKB]

Cahill, L., Uncapher, M., Kilpatrick, L., Alkire, M. T. \& Turner, J. (2004) Sex-related hemispheric lateralization of amygdala function in emotionally influenced memory: An fMRI investigation. Learning and Memory 11: 261-66. [aBC]

Caligiuri, M. P., Hellige, J. B., Cherry, B. J., Kwok, W., Lulow, L. L. \& Lohr, J. B. (2005) Lateralized cognitive dysfunction and psychotic symptoms in schizophrenia. Schizophrenia Research 80:151-61. [aBC]

Camisa, K. M., Bockbrader, M. A., Lysaker, P., Rae, L. L., Brenner, C. A. \& O'Donnell, B. F. (2005) Personality traits in schizophrenia and related personality disorders. Psychiatry Research 133:23-33. [aBC] 
Cannon, M., Jones, P. B., Gilvarry, C., Rifkin, L., McKenzie, K., Foerster, A. \& Murray, R. M. (1997) Premorbid social functioning in schizophrenia and bipolar disorder: Similarities and differences. American Journal of Psychiatry 154:1544-50. [aBC]

Cannon, M., Jones, P. B. \& Murray, R. M. (2002) Obstetric complications and schizophrenia: Historical and meta-analytic review. American Journal of Psychiatry 159:1080-92. [aBC]

Cannon, T. D. \& Keller, M. C. (2006) Endophenotypes in the genetic analyses of mental disorders. Annual Review of Clinical Psychology 2:267-90. $[\mathrm{rBC}]$

Cannon, T. D., Rosso, I. M., Hollister, J. M., Bearden, C. E., Sanchez, L. E. \& Hadley, T. (2000) A prospective cohort study of genetic and perinatal influences in the etiology of schizophrenia. Schizophrenia Bulletin 26 : 351-66. [aBC]

Cantor, R. M., Yoon, J. L., Furr, J. \& Lajonchere, C. M. (2007) Paternal age and autism are associated in a family-based sample. Molecular Psychiatry 12:419-21. [rBC]

Cantor, S., Evans, J., Pearce, J. \& Pezzot-Pearce, T. (1982) Childhood schizophrenia: Present but not accounted for. American Journal of Psychiatry 139:758-62. [ST]

Capps, L., Kasari, C., Yirmiya, N. \& Sigman, M. (1993) Parental perception of emotional expressiveness in children with autism. Journal of Consulting and Clinical Psychology 61:475-84. [aBC]

Carpenter, W. T., Heinrichs, D. W. \& Wagman, A. M. (1988) Deficit and nondeficit forms of schizophrenia: The concept. American Journal of Psychiatry 145:578-83. [ST]

Carper, R. A., Moses, P., Tigue, Z. D. \& Courchesne, E. (2002) Cerebral lobes in autism: Early hyperplasia and abnormal age effects. NeuroImage 16:1038-51. [aBC]

Carroll, J. M. \& Chiew, K. Y. (2006) Sex and discipline differences in empathising, systemising and autistic symptomatology: Evidence from a student population. Journal of Autism and Developmental Disorders 36:949-57. [aBC]

Carruthers, P. (2002) The cognitive functions of language. Behavioral and Brain Sciences 25:657-74. [WF]

Carter, C. S. \& Altemus, M. (1997) Integrative functions of lactational hormones in social behavior and stress management. Annals of the New York Academy of Sciences 807:164-74. [LMG]

Carter, C. S., Robertson, L. C., Nordahl, T. E., Chaderjian, M. \& Oshora-Celaya, L. (1996) Perceptual and attentional asymmetries in schizophrenia: Further evidence for a left hemisphere deficit. Psychiatry Research 62:111-19. $[\mathrm{aBC}]$

Cascio, C., McGlone, F., Folger, S., Tannan, V., Baranek, G., Pelphrey, K. A. \& Essick, G. (2008) Tactile perception in adults with autism: A multidimensional psychophysical study. Journal of Autism and Developmental Disorders 38:127-37. [aBC]

Castellanos, F. X. \& Tannock, R. (2002) Neuroscience of attention-deficit/ hyperactivity disorder: The search for endophenotypes. Nature Reviews Neuroscience 3(8):617-28. [LMG]

Castelli, F. (2005) Understanding emotions from standardized facial expressions in autism and normal development. Autism 9:428-49. [aBC]

Castelli, F., Frith, C., Happé, F. \& Frith, U. (2002) Autism, Asperger syndrome and brain mechanisms for the attribution of mental states to animated shapes. Brain: A Journal of Neurology 125:1839-49. [aBC, RL]

Catalano, P. M. \& Kirwan, J. P. (2001) Maternal factors that determine neonatal size and body fat. Current Diabetes Reports 1:71-77. [aBC]

Cattanach, B. M., Beechey, C. V. \& Peters, J. (2004) Interactions between imprinting effects in the mouse. Genetics 168:397-413. [aBC]

Cederlund, M. \& Gillberg, C. (2004) One hundred males with Asperger syndrome: A clinical study of background and associated factors. Developmental Medicine and Child Neurology 46:652-60. [aBC]

Cerrato, F., Sparago, A., Di Matteo, I., Zou, X., Dean, W., Sasaki, H., Smith, P., Genesio, R., Bruggemann, M., Reik, W. \& Riccio, A. (2005) The two-domain hypothesis in Beckwith-Wiedemann syndrome: Autonomous imprinting of the telomeric domain of the distal chromosome 7 cluster. Human Molecular Genetics 14:503-11. [aBC]

Chance, M. R. A. \& Mead, A. P. (1953) Social behaviour and primate evolution. Symposia of the Society for Experimental Biology 7:395-439. [aBC]

Chance, S. A., Esiri, M. M. \& Crow, T. J. (2005) Macroscopic brain asymmetry is changed along the antero-posterior axis in schizophrenia. Schizophrenia Research 74:163-70. [aBC]

Chandana, S. R., Behen, M. E., Juhasz, C., Muzik, O., Rothermel, R. D., Mangner, T. J., Chakraborty, P. K., Chugani, H. T. \& Chugani, D. C. (2005) Significance of abnormalities in developmental trajectory and asymmetry of cortical serotonin synthesis in autism. International Journal of Developmental Neuroscience $23(2-3): 171-82$. [LMG]

Charalambous, M., Smith, F. M., Bennett, W. R., Crew, T. E., Mackenzie, F. \& Ward, A. (2003) Disruption of the imprinted GRB10 gene leads to disproportionate overgrowth by an IGF2-independent mechanism. Proceedings of the National Academy of Sciences USA 100:8292-97. [aBC]

Charman, T. (1997) The relationship between joint attention and pretend play in autism. Development and Psychopathology 9:1-16. [MKB]

Chen, F., Planche, P., Lemonnier, E. \& Lazartigues, A. (2007) How could language interact with visuo-spatial performance in autism? Medical Hypotheses 69:695-97. [aBC]

Chen, M., Haluzik, M., Wolf, N. J., Lorenzo, J., Dietz, K. R., Reitman, M. L. \& Weinstein, L. S. (2004) Increased insulin sensitivity in paternal GNAS knockout mice is associated with increased lipid clearance. Endocrinology 145:4094-4102. [aBC]

Chen, Y., Bidwell, L. C. \& Holzman, P. S. (2005) Visual motion integration in schizophrenia patients, their first-degree relatives, and patients with bipolar disorder. Schizophrenia Research 74:271-81. [aBC]

Cheney, D., Seyfarth, R. \& Smuts, B. (1986) Social relationships and social cognition in nonhuman primates. Science 234(4782):1361-66. [LMG]

Cheng, M. C. \& Chen, C. H. (2007) Identification of rare mutations of synaptogyrin 1 gene in patients with schizophrenia. Journal of Psychiatric Research 41(12):1027-31. [MCK

Cheng, Y., Tzeng, O. J. L., Decety, J., Imada, T. \& Hsieh, J. (2006) Gender differences in the human mirror system: A magnetoencephalography study. Neuroreport 17:1115-19. [aBC]

Chi, J. G., Dooling, E. C. \& Gilles, F. H. (1977) Gyral development of the human brain. Annals of Neurology 1:86-93. [aBC]

Chiron, C., Leboyer, M., Leon, F., Jambaqué, I., Nuttin, C. \& Syrota, A. (1995) SPECT of the brain in childhood autism: Evidence for a lack of normal hemispheric asymmetry. Developmental Medicine and Child Neurology 37:849-60. [aBC]

Chiu, P. H., Kayali, M. A., Kishida, K. T., Tomlin, D., Klinger, L. G., Klinger, M. R. \& Montague, P. R. (2008) Self responses along cingulate cortex reveal quantitative neural phenotype for high-functioning autism. Neuron $57: 463-73 . \quad[\mathrm{rBC}]$

Chiu, S., Wegelin, J. A., Blank, J., Jenkins, M., Day, J., Hessl, D., Tassone, F. \& Hagerman, R. (2007) Early acceleration of head circumference in children with Fragile X syndrome and autism. Journal of Developmental and Behavioral Pediatrics 28:31-5. [rBC]

Christian, S. L., Fantes, J. A., Mewborn, S. K., Huang, B. \& Ledbetter, D. H. (1999) Large genomic duplicons map to sites of instability in the Prader-Willi/ Angelman syndrome chromosome region (15q11-q13). Human Molecular Genetics 8(6):1025-37. [CKD]

Christoff, K., Ream, J. M. \& Gabrieli, J. D. (2004) Neural basis of spontaneous thought processes. Cortex 40:623-30. [aBC]

Chua, S. E., Wright, I. C., Poline, J. B., Liddle, P. F., Murray, R. M., Frackowiak, R. S., Friston, K. J. \& McGuire, P. K. (1997) Grey matter correlates of syndromes in schizophrenia: A semi-automated analysis of structural magnetic resonance images. British Journal of Psychiatry 170:406-10. [aBC]

Chubb, J. E., Bradshaw, N. J., Soares, D. C., Porteous, D. J. \& Millar, J. K. (2008) The DISC locus in psychiatric illness. Molecular Psychiatry 13:36-64. [rBC]

Claridge, G. (1997) Schizotypy: Implications for illness and health. Oxford University Press. [aBC]

Claridge, G. \& Beech, T. (1995) Fully and quasi-dimensional constructions of schizotypy. In: Schizotypal personality, ed. A. Raine, T. Lencz \& S. A. Mednick, pp. 192-216. Cambridge University Press. [aBC]

Claridge, G., Clark, K. \& Davis, C. (1997) Nightmares, dreams, and schizotypy. British Journal of Clinical Psychology 36:377-86. [aBC]

Claridge, G., Pryor, R. \& Watkins, G. (1990) Sounds from the bell jar: Ten psychotic authors. Macmillan. [arBC]

Clegg, H. (2006) Creativity: Psychological and evolutionary perspectives. Doctoral thesis. The Open University. [DN]

Clifford, S., Dissanayake, C., Bui, Q. M., Huggins, R., Taylor, A. K. \& Loesch, D. Z. (2007) Autism spectrum phenotype in male and females with fragile X full mutation and permutation. Journal of Autism and Developmental Disorders 37(4):738-47. [CKD]

Coan, P. M., Burton, G. J. \& Ferguson-Smith, A. C. (2005) Imprinted genes in the placenta - A review. Placenta 26(Suppl. A):S10-20. [arBC]

Cody, H., Pelphrey, K. \& Piven, J. (2002) Structural and functional magnetic resonance imaging of autism. International Journal of Developmental Neuroscience 20:421-38. [aBC]

Cohen, D., Martel, C., Wilson, A., Déchambre, N., Amy, C., Duverger, L., Guile, J., Pipiras, E., Benzacken, B., Cavé, H., Cohen, L., Héron, D. \& Plaza, M. (2007) Brief report: Visual-spatial deficit in a 16-year-old girl with maternally derived duplication of proximal 15q. Journal of Autism and Developmental Disorders 37:1585-91. [rBC]

Cohen, D., Pichard, N., Tordjman, S., Baumann, C., Burglen, L., Excoffier, E., Lazar, G., Mazet, P., Pinquier, C., Verloes, A. \& Héron, D. (2005) Specific genetic disorders and autism: Clinical contribution towards their identification. Journal of Autism and Developmental Disorders 35:103-16. $[\mathrm{aBC}]$ 
Cohen-Bendahan, C. C. C., van de Beek, C. \& Berenbaum, S. A. (2005) Prenatal sex hormone effects on child and adult sex-typed behavior: Methods and findings. Neuroscience and Biobehavioral Reviews 29:353-84. [MV]

Collinson, S. L., Mackay, C. E., James, A. C., Quested, D. J., Phillips, T., Roberts, N. \& Crow, T. J. (2003) Brain volume, asymmetry and intellectual impairment in relation to sex in early-onset schizophrenia. British Journal of Psychiatry 183:114-20. [aBC]

Compton, M. T., Celentana, M., Price, B. \& Furman, A. C. (2004) A case of Sotos syndrome (cerebral gigantism) and psychosis. Psychopathology 37:190-93. $[\mathrm{rBC}]$

Condray, R. (2005) Language disorder in schizophrenia as a developmental learning disorder. Schizophrenia Research 73:5-20. [aBC]

Connolly, A. M., Chez, M., Streif, E. M., Keeling, R. M., Golumbek, P. T., Kwon, J. M., Riviello, J. J., Robinson, R. G., Neuman, R. J. \& Deuel, R. M. (2006) Brain-derived neurotrophic factor and autoantibodies to neural antigens in sera of children with autistic spectrum disorders, Landau-Kleffner syndrome, and epilepsy. Biological Psychiatry 59:354-63. [aBC]

Constantino, J. N. \& Todd, R. D. (2005) Intergenerational transmission of subthreshold autistic traits in the general population. Biological Psychiatry 57:655-60. [aBC]

Conti-Ramsden, G., Simkin, Z. \& Botting, N. (2006) The prevalence of autistic spectrum disorders in adolescents with a history of specific language impairment (SLI). Journal of Child Psychology and Psychiatry, and Allied Disciplines 47:621-28. [aBC]

Cook, E. H., Jr., Lindgren, V., Leventhal, B. L., Courchesne, R., Lincoln, A., Shulman, C., Lord, C. \& Courchesne, E. (1997) Autism or atypical autism in maternally but not paternally derived proximal $15 \mathrm{q}$ duplication. American Journal of Human Genetics 60:928-34. [rBC]

Corbett, B. A. \& Constantine, L. J. (2006) Autism and attention deficit hyperactivity disorder: Assessing attention and response control with the integrated visual and auditory continuous performance test. Child Neuropsychology 12:33548. $[\mathrm{MKB}]$

Corbett, B. A., Mendoza, S., Abdullah, M., Wegelin, J. A. \& Levine, S. (2006) Cortisol circadian rhythms and response to stress in children with autism. Psychoneuroendocrinology 31(1):59-68. [LMG]

Corcoran, R. \& Frith, C. D. (1996) Conversational conduct and the symptoms of schizophrenia. Cognitive Neuropsychiatry 1:305-18. [AT]

Costello, E. J., Worthman, C., Erkanli, A. \& Angold, A. (2007) Prediction from low birth weight to female adolescent depression: A test of competing hypotheses. Archives of General Psychiatry 64:338-44. [aBC]

Courchesne, E. (2004) Brain development in autism: Early overgrowth followed by premature arrest of growth. Mental Retardation and Developmental Disabilities Research Reviews 10:106-11. [aBC]

Courchesne, E. \& Pierce, K. (2005a) Brain overgrowth in autism during a critical time in development: Implications for frontal pyramidal neuron and interneuron development and connectivity. International Journal of Developmental Neuroscience 23:153-70. [aBC]

(2005b) Why the frontal cortex in autism might be talking only to itself: Local over-connectivity but long-distance disconnection. Current Opinion in Neurobiology 15:225-30. [aBC]

Courchesne, E., Redcay, E. \& Kennedy, D. P. (2004) The autistic brain: Birth through adulthood. Current Opinion in Neurology 17:489-96. [aBC]

Courchesne, E., Townsend, J., Akshoomoff, N. A., Saitoh, O., Yeung-Courchesne, R., Lincoln, A. J., James, H. E., Haas, R. H., Schreibman, L. \& Lau, L. (1994) Impairment in shifting attention in autistic and cerebellar patients. Behavioral Neuroscience 108:848-65. [aBC]

Craddock, N. \& Forty, L. (2006) Genetics of affective (mood) disorders. European Journal of Human Genetics 14:660-68. [aBC]

Craddock, N., O’Donovan, M. C. \& Owen, M. J. (2005) The genetics of schizophrenia and bipolar disorder: Dissecting psychosis. Journal of Medical Genetics 42:193-204. [rBC]

Craig, J. \& Baron-Cohen, S. (1999) Creativity and imagination in autism and Asperger syndrome. Journal of Autism and Developmental Disorders 29:319-26. [aBC]

Craig, J. S., Hatton, C., Craig, F. B. \& Bentall, R. P. (2004) Persecutory beliefs, attributions and theory of mind: Comparison of patients with paranoid delusions, Asperger's syndrome and healthy controls. Schizophrenia Research 69:29-33. [arBC, SvR]

Crawley, J. N. (2007) Mouse behavioral assays relevant to the symptoms of autism. Brain Pathology 17(4):448-59. [THJB]

Crespi, B. J. (2000) The evolution of maladaptation. Heredity 84:623-29. [aBC]

(2006) The natural selection of psychosis. Behavioral and Brain Sciences 29:410-11. [arBC]

(2007) Sly FOXP2: Genomic conflict in the evolution of language. Trends in Ecology and Evolution 22:174-75. [rBC]

(under revision) Genomic imprinting in the development and evolution of psychosis. Biological Reviews. [rBC] (in review) Genomic sister-disorders of growth, development, and cognition. BMC Medical Genomics. [rBC]

(in press a) Language unbound: Genomic conflict and psychosis in the origin of modern humans. In: Social communication, ed. D. Hughes \& P. D’Ettorre. Oxford University Press. [rBC]

(in press b) Turner syndrome and the evolution of human sexual dimorphism. Evolutionary Applications. [rBC]

Crespi, B. \& Semeniuk, C. (2004) Parent-offspring conflict in the evolution of vertebrate reproductive mode. American Naturalist 163:635-53. [aBC]

Crespi, B., Summers, K. \& Dorus, S. (2007) Adaptive evolution of genes underlying schizophrenia. Proceedings of the Royal Society B: Biological Sciences 274:2801-810. [aBC]

Crosbie, J., Perusse, D., Barr, C. L. \& Schachar, R. J. (2008) Validating psychiatric endophenotypes: Inhibitory control and attention deficit hyperactivity disorder. Neuroscience and Biobehavioral Reviews $32(1): 40-55$. [LMG]

Crow, J. F. (2000) The origins, patterns and implications of human spontaneous mutation. Nature Reviews. Genetics 1(1):40-47. [ТНJB]

Crow, T. J. (1995) A Darwinian approach to the origins of psychosis. British Journal of Psychiatry 167:12-25. [aBC]

(1997) Schizophrenia as failure of hemispheric dominance for language. Trends in Neurosciences 20:339-43. [aBC]

(1998) From Kraepelin to Kretschmer leavened by Schneider: The transition from categories to psychosis to dimensions of variation intrinsic to Homo sapiens. Archives of General Psychiatry 55:502-504. [aBC]

(2000) Schizophrenia as the price that Homo sapiens pays for language: A resolution of the central paradox in the origin of the species. Brain Research Reviews 31:118-29. [aBC]

(2004a) Auditory hallucinations as primary disorders of syntax: An evolutionary theory of the origins of language. Cognitive Neuropsychiatry 9:125-45. $[\mathrm{aBC}]$

(2004b) Cerebral asymmetry and the lateralization of language: Core deficits in schizophrenia as pointers to the gene. Current Opinion in Psychiatry 17:97-106. [aBC]

(2004c) What Marian Annett can teach Noam Chomsky and could have taught Stephen Jay Gould if he'd had time to listen. Cortex 40:120-34. [aBC]
[all

Crow, T. J., Crow, L. R., Done, D. J. \& Leask, S. (1998) Relative hand skill predicts academic ability: Global deficits at the point of hemispheric indecision. Neuropsychologia 36:1275-82. [aBC]

Crow, T. J., Done, D. J. \& Sacker, A. (1996) Cerebral lateralization is delayed in children who later develop schizophrenia. Schizophrenia Research 22:181-85. $\quad[\mathrm{aBC}]$

Cunnane, S. C. \& Crawford, M. A. (2003) Survival of the fattest: Fat babies were the key to evolution of the large human brain. Comparative Biochemistry and Physiology. Part A, Molecular and Integrative Physiology 136:17-26. [aBC]

Curley, J. P., Barton, S., Surani, A. \& Keverne, E. B. (2004) Coadaptation in mother and infant regulated by a paternally expressed imprinted gene. Proceedings of the Royal Society of London, Series B: Biological Sciences 271:1303-309. $[\mathrm{aBC}]$

Dalton, K. M., Nacewicz, B. M., Johnstone, T., Schaefer, H. S., Gernsbacher, M. A., Goldsmith, H. H., Alexander, A. L. \& Davidson, R. J. (2005a) Gaze fixation and the neural circuitry of face processing in autism. Nature Neuroscience 8:51926. [aBC]

Dalton, S. O., Mellemkjaer, L., Thomassen, L., Mortensen, P. B. \& Johansen, C. (2005b) Risk for cancer in a cohort of patients hospitalized for schizophrenia in Denmark, 1969-1993. Schizophrenia Research 75:315-24. [aBC]

Dan, B. \& Boyd, S. G. (2003) Angelman syndrome reviewed from a neurophysiological perspective. The UBE3A-GABRB3 hypothesis. Neuropediatrics 34:169-76. [aBC]

Dapretto, M., Davies, M. S., Pfeifer, J. H., Scott, A. A., Sigman, M., Bookheimer, S. Y. \& Iacoboni, M. (2006) Understanding emotions in others: Mirror neuron dysfunction in children with autism spectrum disorders. Nature Neuroscience 9:28-30. [aBC]

Das, P., Kemp, A. H., Flynn, G., Harris, A. W. F., Liddell, B. J., Whitford, T. J., Peduto, A., Gordon, E. \& Williams, L. M. (2007) Functional disconnections in the direct and indirect amygdala pathways for fear processing in schizophrenia. Schizophrenia Research 90:284-94. [aBC]

Davies, W., Isles, A. R., Burgoyne, P. S. \& Wilkinson, L. S. (2006) X-linked imprinting: Effects on brain and behaviour. Bioessays 28:35-44. [aBC]
[äe

Davies, W., Isles, A. R., Smith, R., Karunadasa, D., Burrmann, D., Humby, T., Ojarikre, O., Biggin, C., Skuse, D., Burgoyne, P. \& Wilkinson, L. (2005) Xlr3b is a new imprinted candidate for $\mathrm{X}$-linked parent-of-origin effects on cognitive function in mice. Nature Genetics 37:625-29. [aBC]

Davies, W., Isles, A. R. \& Wilkinson, L. S. (2001) Imprinted genes and mental dysfunction. Annals of Medicine 33:428-36. [aBC]

(2005) Imprinted gene expression in the brain. Neuroscience and Biobehavioral Reviews 29:421-30. [BJAD] 
Davis, J. O., Phelps, J. A. \& Bracha, H. S. (1995) Prenatal development of monozygotic twins and concordance for schizophrenia. Schizophrenia Bulletin 21:357-66. [aBC]

Davis, P. J. \& Gibson, M. G. (2000) Recognition of posed and genuine facial expressions of emotion in paranoid and nonparanoid schizophrenia. Journal of Abnormal Psychology 109:445-50. [aBC]

Dawson, M., Soulières, I., Gernsbacher, M. A. \& Mottron, L. (2007) The level and nature of autistic intelligence. Psychological Science 18:657-62. [rBC]

Day, T. \& Bonduriansky, R. (2004) Intralocus sexual conflict can drive the evolution of genomic imprinting. Genetics 167:1537-46. [BJAD]

de Boer, L., Röder, I. \& Wit, J. M. (2006) Psychosocial, cognitive, and motor functioning in patients with suspected Sotos syndrome: A comparison between patients with and without NSD1 gene alterations. Developmental Medicine and Child Neurology 48:582-88. [rBC]

de Bruin, E. I., Verheij, F., Wiegman, T. \& Ferdinand, R. F. (2006) Differences in finger length ratio between males with autism, pervasive developmental disorder-not otherwise specified, ADHD, and anxiety disorders. Developmental Medicine and Child Neurology 48:962-65. [MV]

De Fossé, L., Hodge, S. M., Makris, N., Kennedy, D. N., Caviness, V. S., Jr., McGrath, L., Steele, S., Ziegler, D. A., Herbert, M. R., Frazier, J. A., Tager-Flusberg, H. \& Harris, G. J. (2004) Language-association cortex asymmetry in autism and specific language impairment. Annals of Neurology 56:757-66. [aBC]

de la Fuente-Sandoval, C., Portillo, V., Fresán, A. \& Apiquian, R. (2005) Replication of a computer model of auditory hallucinations in schizophrenia. Actas Españolas De Psiquiatría 33:141-46. [aBC]

De Luca, V., Wong, A. H. C., Muller, D. J., Wong, G. W. H., Tyndale, R. F. \& Kennedy, J. L. (2004) Evidence of association between smoking and alpha7 nicotinic receptor subunit gene in schizophrenia patients. Neuropsychopharmacology 29:1522-26. [aBC]

DeLisi, L. E., Maurizio, A. M., Svetina, C., Ardekani, B., Szulc, K., Nierenberg, J., Leonard, J. \& Harvey, P. D. (2005) Klinefelter's syndrome (XXY) as a genetic model for psychotic disorders. American Journal of Medical Genetics. Part B, Neuropsychiatric Genetics 135:15-23. [arBC]

DeLisi, L. E., Svetina, C., Razi, K., Shields, G., Wellman, N. \& Crow, T. J. (2002) Hand preference and hand skill in families with schizophrenia. Laterality $7: 321-32$. [aBC]

DeLong, R. (2004) Autism and familial major mood disorder: Are they related? Journal of Neuropsychiatry and Clinical Neuroscience 16:199-213. [MKB]

(2007) GABA(A) receptor alpha5 subunit as a candidate gene for autism and bipolar disorder: A proposed endophenotype with parent-of-origin and gain-of-function features, with or without oculocutaneous albinism. Autism $11(2): 135-47 . \quad[\mathrm{GV}]$

Demaree, H. A., Everhart, D. E., Youngstrom, E. A. \& Harrison, D. W. (2005) Brain lateralization of emotional processing: Historical roots and a future incorporating "dominance." Behavioral and Cognitive Neuroscience Reviews $4: 3-20$. [R-PB]

Deng, Y., Bhattacharya, S., Swamy, O. R., Tandon, R., Wang, Y., Janda, R. \& Riedel, H. (2003) Growth factor receptor-binding protein 10 (GRB10) as a partner of phosphatidylinositol 3-kinase in metabolic insulin action. Journal of Biological Chemistry 278:39311-22. [aBC]

Dennis, M., Lockyer, L., Lazenby, A. L., Donnelly, R. E., Wilkinson, M. \& Schoonheyt, W. (1999) Intelligence patterns among children with highfunctioning autism, phenylketonuria, and childhood head injury. Journal of Autism and Developmental Disorders 29:5-17. [rBC]

Depienne, C., Heron, D., Betancur, C., Benyahia, B., Trouillard, O., Bouteiller, D., Verloes, A., LeGuern, E., Leboyer, M. \& Brice, A. (2007) Autism, language delay and mental retardation in a patient with $7 q 11$ duplication. Journal of Medical Genetics 44:452-58. [rBC]

Derry, C., Temple, I. K. \& Venkat-Raman, K. (1999) A probable case of familial Weaver syndrome associated with neoplasia. Journal of Medical Genetics 36:725-28. [rBC]

Deutsch, C. K. (1998) Emergent properties of brain development and function. In: Perspectives on fundamental processes in intellectual functioning: A survey of research approaches, vol. 2, ed. S. A. Soraci \& W. J. McIlvane, pp. 168-85. Ablex. [CKD]

Devriendt, K. (2005) Hydatidiform mole and triploidy: The role of genomic imprinting in placental development. Human Reproduction Update 11:137-42. [aBC]

DiCicco-Bloom, E., Lord, C., Zwaigenbaum, L., Courchesne, E., Dager, S. R., Schmitz, C., Schultz, R. T., Crawley, J. \& Young, L. J. (2006) The developmental neurobiology of autism spectrum disorder. The Journal of Neuroscience 26:6897-6906. [aBC]

Dimitropoulos, A. \& Schultz, R. T. (2007) Autistic-like symptomatology in Prader-Willi syndrome: A review of recent findings. Current Psychiatry Reports 9(2):159-64. [WD]

Dinn, W. M., Harris, C. L., Aycicegi, A., Greene, P. \& Andover, M. S. (2002) Positive and negative schizotypy in a student sample: Neurocognitive and clinical correlates. Schizophrenia Research 56:171-85. [aBC]
Dissanayake, C., Bui, Q. M., Huggins, R. \& Loesch, D. Z. (2006) Growth in stature and head circumference in high-functioning autism and Asperger disorder during the first 3 years of life. Development and Psychopathology 18:381-93. [aBC]

Dobzhansky, T. (1973) Nothing in biology makes sense except in the light of evolution. The American Biology Teacher 35:125-29. [GV]

Dolinoy, D. C., Weidman, J. R. \& Jirtle, R. L. (2006) Epigenetic gene regulation: Linking early developmental environment to adult disease. Reproductive Toxicology 23:297-307. [aBC]

Dollfus, S., Razafimandimby, A., Delamillieure, P., Brazo, P., Joliot, M., Mazoyer, B. \& Tzourio-Mazoyer, N. (2005) Atypical hemispheric specialization for language in right-handed schizophrenia patients. Biological Psychiatry $57: 1020-28 . \quad[\mathrm{aBC}]$

Douglas, J., Cilliers, D., Coleman, K., Tatton-Brown, K., Barker, K., Bernhard, B., Burn, J., Huson, S., Josifova, D., Lacombe, D., Malik, M., Mansour, S., Reid, E., Cormier-Daire, V., Cole, T. \& Rahman, N. (2007) Mutations in RNF135, a gene within the NF1 microdeletion region, cause phenotypic abnormalities including overgrowth. Nature Genetics 39:963-65. [rBC]

Downhill, J. E., Jr., Buchsbaum, M. S., Wei, T., Spiegel-Cohen, J., Hazlett, E. A., Haznedar, M. M., Silverman, J. \& Siever, L. J. (2000) Shape and size of the corpus callosum in schizophrenia and schizotypal personality disorder. Schizophrenia Research 42:193-208. [aBC]

Downs, A. \& Smith, T. (2004) Emotional understanding, cooperation, and social behavior in high-functioning children with autism. Journal of Autism and Developmental Disorders 34:625-35. [aBC]

Doyle, T. F., Bellugi, U., Korenberg, J. R. \& Graham, J. (2004) "Everybody in the world is my friend" hypersociability in young children with Williams syndrome. American Journal of Medical Genetics: Part A 124:263-73. [rBC]

Dressler, S. G., Voracek, M. \& Manning, J. T. (2004) 2D:4D, assortative mating, and heritability: Evidence from Austrian family data. Paper presented at the 16th Annual Meeting of the Human Behavior and Evolution Society, July 21-25, 2004, Berlin, Germany. [MV]

Drury, V. M., Robinson, E. J. \& Birchwood, M. (1998) "Theory of mind" skills during an acute episode of psychosis and following recovery. Psychological Medicine 28:1101-12. [rBC]

Dunger, D. B., Petry, C. J. \& Ong, K. K. (2006) Genetic variations and normal fetal growth. Hormone Research 65(3):34-40. [aBC]

Durand, C. M., Betancur, C., Boeckers, T. M., Bockmann, J., Chaste, P., Fauchereau, F., Nygren, G., Rastam, M., Gillberg, I. C., Anckarsater, H., Sponheim, E., Goubran-Botros, H., Delorme, R., Chabane, N., MourenSimeoni, M. C., de Mas, P., Bieth, E., Roge, B., Heron, D., Burglen, L., Gillberg, C., Leboyer, M. \& Bourgeron, T. (2007) Mutations in the gene encoding the synaptic scaffolding protein SHANK3 are associated with autism spectrum disorders. Nature Genetics 39(1):25-27. [MCK]

Dutta, S., Guhathakurta, S., Sinha, S., Chatterjee, A., Ahmed, S., Ghosh, S., Gangopadhyay, P. K., Singh, M. \& Usha, R. (2007) Reelin gene polymorphisms in the Indian population: A possible paternal 5'UTR-CGG-repeat-allele effect on autism. American Journal of Medical Genetics. Part B, Neuropsychiatric Genetics 144:106-12. [rBC]

Dykens, E. M. (2000) Are jigsaw puzzle skills "spared" in persons with Prader-Willi syndrome? Journal of Child Psychology and Psychiatry 43:343-52. [rBC, $\mathrm{DH}]$

(2003) Anxiety, fears, and phobias in persons with Williams syndrome. Developmental Neuropsychology 23:291-316. [rBC]

Dykens, E. M., Finucane, B. M. \& Gayley, C. (1997) Brief report: Cognitive and behavioral profiles in persons with Smith-Magenis syndrome. Journal of Autism and Developmental Disorders 27:203-11. [rBC]

Dykens, E. M., Hodapp, R. M. \& Finucane, B. M. (2000) Genetics and mental retardation syndromes: A new look at behavior and interventions. Brookes. $[\mathrm{aBC}]$

Dykens, E. M., Sutcliffe, J. S. \& Levitt, P. (2004) Autism and 15q11-q13 disorders: Behavioral, genetic, and pathophysiological issues. Mental Retardation and Developmental Disabilities Research Reviews 10:284-91. [ [rBC]

Dykes, M. \& McGhie, A. (1976) A comparative study of attentional strategies of schizophrenic and highly creative normal subjects. British Journal of Psychiatry 128:50-56. [aBC]

Edelmann, L., Prosnitz, A., Pardo, S., Bhatt, J., Cohen, N., Lauriat, T., Ouchanov, L., González, P. J., Manghi, E. R., Bondy, P., Esquivel, M., Monge, S., Delgado, M. F., Splendore, A., Francke, U., Burton, B. K. \& McInnes, L. A. (2007) An atypical deletion of the Williams-Beuren syndrome interval implicates genes associated with defective visuospatial processing and autism. Journal of Medical Genetics 44:136-43. [rBC]

Edgar, J. C., Yeo, R. A., Gangestad, S. W., Blake, M. B., Davis, J. T., Lewine, J. D. \& Cañive, J. M. (2006) Reduced auditory M100 asymmetry in schizophrenia and dyslexia: Applying a developmental instability approach to assess atypical brain asymmetry. Neuropsychologia 44:289-99. [aBC]

Egaas, B., Courchesne, E. \& Saitoh, O. (1995) Reduced size of corpus callosum in autism. Archives of Neurology 52:794-801. [aBC] 
Eggermann, T., Meyer, E., Obermann, C., Heil, I., Schüler, H., Ranke, M. B., Eggermann, K. \& Wollmann, H. A. (2005) Is maternal duplication of $11 \mathrm{p} 15$ associated with Silver-Russell syndrome? Journal of Medical Genetics 42:e26. [aBC]

Eggermann, T., Schönherr, N., Meyer, E., Obermann, C., Mavany, M., Eggermann, K., Ranke, M. B. \& Wollmann, H. A. (2006) Epigenetic mutations in 11p15 in Silver-Russell syndrome are restricted to the telomeric imprinting domain. Journal of Medical Genetics 43:615-16. [aBC]

Eliez, S. (2007) Autism in children with 22q11.2 deletion syndrome. Journal of the American Academy of Child and Adolescent Psychiatry 46:433-34. [aBC]

Eliez, S. \& van Amelsvoort, T. (2005) Neuroimaging in velo-cardio-facial syndrome. In: Velo-cardio-facial syndrome: A model for understanding microdeletion disorders ed. K. C. Murphy \& P. J. Scambler, pp. 165-80. Cambridge University Press. $[\mathrm{aBC}]$

Ellison, Z., van Os, J. \& Murray, R. (1998) Special feature: Childhood personality characteristics of schizophrenia: Manifestations of, or risk factors for, the disorder? Journal of Personality Disorders 12:247-61. [aBC]

El-Saadi, O., Pedersen, C. B., McNeil, T. F., Saha, S., Welham, J., O’Callaghan, E., Cantor-Graae, E., Chant, D., Mortensen, P. B. \& McGrath J. (2004) Paternal and maternal age as risk factors for psychosis: Findings from Denmark, Sweden and Australia. Schizophrenia Research 67(2-3):227-36. [THJB]

Emamian, E. S., Hall, D., Birnbaum, M. J., Karayiorgou, M. \& Gogos, J. A. (2004) Convergent evidence for impaired AKTl-GSK3beta signaling in schizophrenia. Nature Genetics 36:131-37. [aBC]

Emanuel, B. S. \& Saitta, S. C. (2007) From microscopes to microarrays: Dissecting recurrent chromosomal rearrangements. Nature Reviews. Genetics 8(11): 869-83. [ТНJB]

Emery, N. J. (2000) The eyes have it: The neuroethology, function and evolution of social gaze. Neuroscience and Biobehavioral Reviews 24:581-604. [aBC]

Endrass, T., Mohr, B. \& Rockstroh, B. (2002) Reduced interhemispheric transmission in schizophrenia patients: Evidence from event-related potentials. Neuroscience Letters 320:57-60. [aBC]

Engelen, J. J., Loots, W. J., Albrechts, J. C., Schrander-Stumpel, C. T., Dirckx, R., Smeets, H. J., Hamers, A. J. \& Geraedts, J. P. (1999) Duplication within chromosome region 15q11-q13 in a patient with similarities to Prader-Willi syndrome confirmed by region-specific and band-specific FISH. Genetic Counseling 10:123-32. [rBC]

Engstrom, H. A., Ohlson, S., Stubbs, E. G., Maciulis, A., Caldwell, V., Odell, J. D. \& Torres, A. R. (2003) Decreased expression of CD95 (Fas/APO-1) on CD4+ T-lymphocytes from participants with autism. Journal of Developmental and Physical Disabilities 15:155-63. [aBC]

Ensenauer, R. E., Adeyinka, A., Flynn, H. C., Michels, V. V., Lindor, N. M., Dawson, D. B., Thorland, E. C., Lorentz, C. P., Goldstein, J. L., McDonald, M. T., Smith, W. E., Simon-Fayard, E., Alexander, A. A., Kulharya, A. S. Ketterling, R. P., Clark, R. D. \& Jalal, S. M. (2003) Microduplication 22q11.2, an emerging syndrome: Clinical, cytogenetic, and molecular analysis of thirteen patients. American Journal of Human Genetics 73:1027-40. $[\mathrm{rBC}]$

Escalante-Mead, P. R., Minshew, N. J. \& Sweeney, J. A. (2003) Abnormal brain lateralization in high-functioning autism. Journal of Autism and Developmental Disorders 33:539-43. [aBC]

Escalona, A., Field, T., Nadel, J. \& Lundy, B. (2002) Brief report: Imitation effects on children with autism. Journal of Autism and Developmental Disorders 32:141-44. [R-PB]

Eyles, D., Brown, J., Mackay-Sim, A., McGrath, J. \& Feron, F. (2003) Vitamin D3 and brain development. Neuroscience 118(3):641-53. [THJB]

Falter, C. M., Plaisted, K. C. \& Davis, G. (in press) Visuo-spatial processing in autism: Testing the predictions of extreme male brain theory. Journal of Autism and Developmental Disorders. DOI:10.1007/s10803-007-0419-8. $[\mathrm{MV}]$

Fañanás, L. \& Bertranpetit, J. (1995) Reproductive rates in families of schizophrenic patients in a case-control study. Acta Psychiatrica Scandinavica 91:202-204. [aBC, MCK]

Fanous, A., Gardner, C., Walsh, D. \& Kendler, K. S. (2001) Relationship between positive and negative symptoms of schizophrenia and schizotypal symptoms in nonpsychotic relatives. Archives of General Psychiatry 58:669-73. [aBC]

Fanous, A. H. \& Kendler, K. S. (2005) Genetic heterogeneity, modifier genes, and quantitative phenotypes in psychiatric illness: Searching for a framework. Molecular Psychiatry 10(1):6-13. [CKD]

Fatemi, S. H., Cuadra, A. E., El Fakahany, E. E., Sidwell, R. W. \& Thuras, P. (2000) Prenatal viral infection causes alterations in nNOS expression in developing mouse brains. Neuroreport 11(7):1493-96. [THJB]

Fatemi, S. H. \& Halt, A. R. (2001) Altered levels of Bcl2 and p53 proteins in parietal cortex reflect deranged apoptotic regulation in autism. Synapse 42:281-84 $[\mathrm{aBC}]$

Fatemi, S. H., Reutiman, T. J., Folsom, T. D., Huang, H., Oishi, K., Mori, S., Smee, D. F., Pearce, D. A., Winter, C., Sohr, R. \& Juckel, G. (2008) Maternal infection leads to abnormal gene regulation and brain atrophy in mouse offspring: Implications for the genesis of neurodevelopmental disorders. Schizophrenia Research 99:56-70. [rBC]

Fatemi, S. H., Reutiman, T. J., Folsom, T. D. \& Sidwell, R. W. (2007) The role of cerebellar genes in pathology of autism and schizophrenia. Cerebellum 1-16. Online article, available at: http://dx.doi.org/10.1080/14734220701392969 [THJB]

Feinstein, C., Eliez, S., Blasey, C. \& Reiss, A. L. (2002) Psychiatric disorders and behavioral problems in children with velocardiofacial syndrome: Usefulness as phenotypic indicators of schizophrenia risk. Biological Psychiatry 51:312-18. [aBC]

Feinstein, C. \& Singh, S. (2007) Social phenotypes in neurogenetic syndromes. Child and Adolescent Psychiatric Clinics of North America 16:631-47. [arBC, LMG]

Feldman, R., Greenbaum, C. W. \& Yirmiya, N. (1999) Mother-infant affect synchrony as an antecedent of the emergence of self-control. Developmental Psychology 35:223-31. [R-PB]

Ferguson, J. N., Young, L. J. \& Insel, T. R. (2002) The neuroendocrine basis of social recognition. Frontiers in Neuroendocrinology 23:200-24. [R-PB]

Fernyhough, C. \& Fradley, E. (2005) Private speech on an executive task: Relations with task difficulty and task performance. Cognitive Development 20:103-20. [WF]

Fidler, D. J., Bailey, J. N. \& Smalley, S. L. (2000) Macrocephaly in autism and other pervasive developmental disorders. Developmental Medicine and Child Neurology 42:737-40. [rBC]

Field, T., Sanders, C. \& Nadel, J. (2001) Children with autism display more social behaviors after repeated imitation sessions. Autism 5:317-23. [R-PB]

Fine, S. E, Weissman, A., Gerdes, M., Pinto-Martin, J., Zackai, E. H., McDonaldMcGinn, D. M., \& Emanuel B. S. (2005) Autism spectrum disorders and symptoms in children with molecularly confirmed 22q11.2 deletion syndrome. Journal of Autism and Developmental Disorders 35(4):461-70. [CKD]

Fineberg, N. A., Saxena, S., Zohar, J. \& Craig, K. J. (2007) Obsessive-compulsive disorder: Boundary issues. CNS Spectrums 12:359-75. [aBC]

Finger, E. C., Marsh, A. A., Kamel, N., Mitchell, D. G. V. \& Blair, J. R. (2006) Caught in the act: The impact of audience on the neural response to morally and socially inappropriate behavior. NeuroImage 33:414-21. [aBC]

Fiore, R., Siegel, G. \& Schratt, G. (in press) MicroRNA function in neuronal development, plasticity and disease. Biochimica et Biophysica Acta. $\quad[\mathrm{rBC}]$

Fisher, J. E., Mohanty, A., Herrington, J. D., Koven, N. S., Miller, G. A. \& Heller, W. (2004) Neuropsychological evidence for dimensional schizotypy: Implications for creativity and psychopathology. Journal of Research in Personality 38:24-31. [aBC]

Fisher, R. A., Hodges, M. D., Rees, H. C., Sebire, N. J., Seckl, M. J., Newlands, E. S., Genest, D. R. \& Castrillon, D. H. (2002) The maternally transcribed gene p57(KIP2) (CDNK1C) is abnormally expressed in both androgenetic and biparental complete hydatidiform moles. Human Molecular Genetics $11: 3267-72 . \quad[\mathrm{aBC}]$

Fitzgerald, M. (2004) Autism and creativity: Is there a link between autism in men and exceptional ability? Brunner-Routledge. [aBC]

(2005) The genesis of artistic creativity: Asperger's syndrome and the arts. Jessica Kingsley. $\quad[\mathrm{arBC}]$

Flagg, E. J., Cardy, J. E., Roberts, W. \& Roberts, T. P. (2005) Language lateralization development in children with autism: Insights from the late field magnetoencephalogram. Neuroscience Letters 386:82-87. [aBC]

Fletcher, P. C., Happé, F., Frith, U., Baker, S. C., Dolan, R. J., Frackowiak, R. S. \& Frith, C. D. (1995) Other minds in the brain: A functional imaging study of "theory of mind" in story comprehension. Cognition 57:109-28. [aBC]

Flor-Henry, P. (1969) Psychosis and temporal lobe epilepsy. A controlled investigation. Epilepsia 10:363-95. [aBC]

Foley, R. A. \& Lee, P. C. (1991) Ecology and energetics of encephalization in hominid evolution. Philosophical Transactions of the Royal Society of London. Series B, Biological Sciences 334:223-31. [aBC]

Folstein, S. E. \& Rosen-Sheidley, B. (2001) Genetics of autism: Complex aetiology for a heterogeneous disorder. Nature Reviews. Genetics 2:943-55. [aBC]

Foster, K. R., Wenseleers, T., Ratnieks, F. L. W. \& Queller, D. C. (2006) There is nothing wrong with inclusive fitness. Trends in Ecology and Evolution 21:599-600. [aBC]

Fowden, A. L., Sibley, C., Reik, W. \& Constancia, M. (2006) Imprinted genes, placental development and fetal growth. Hormone Research 65(Suppl. 3):50-58. [arBC]

Fox, R., Sinatra, R. B., Mooney, M. A., Feurer, I. D. \& Butler, M. G. (1999) Visual capacity and Prader-Willi syndrome. Journal of Pediatric Ophthalmology and Strabismus 36:331-36. [aBC]

Franck, N., Daprati, E., Michel, F., Saoud, M., Daléry, J., Marie-Cardine, M. \& Georgieff, N. (1998) Gaze discrimination is unimpaired in schizophrenia. Psychiatry Research 81:67-75. [aBC]

Francks, C., DeLisi, L. E., Fisher, S. E., Laval, S. H., Rue, J. E., Stein, J. F. \& Monaco, A. P. (2003a) Confirmatory evidence for linkage of relative hand skill to 2p12-q11. American Journal of Human Genetics 72:499-502. [aBC] 
Francks, C., DeLisi, L. E., Shaw, S. H., Fisher, S. E., Richardson, A. J., Stein, J. F. \& Monaco, A. P. (2003b) Parent-of-origin effects on handedness and schizophrenia susceptibility on chromosome 2p12-q11. Human Molecular Genetics 12:3225-30. [aBC]

Francks, C., Maegawa, S., Lauren, J., Abrahams, B. S., Velayos-Baeza, A., Medland, S. E., Colella, S., Groszer, M., McAuley, E. Z., Caffrey, T. M., Timmusk, T., Pruunsild, P., Koppel, I., Lind, P. A., Matsumoto-Itaba, N., Nicod, J., Xiong, L., Joober, R., Enard, W., Krinsky, B., Nanba, E., Richardson, A. J., Riley, B. P., Martin, N. G., Strittmatter, S. M., Moller, H. J., Rujescu, D., St Clair, D., Muglia, P., Roos, J. L., Fisher, S. E., Wade-Martins, R., Rouleau, G. A., Stein, J. F., Karayiorgou, M., Geschwind, D. H., Ragoussis, J., Kendler, K. S., Airaksinen, M. S., Oshimura, M., Delisi, L. E. \& Monaco, A. P. (2007) LRRTM1 on chromosome 2p12 is a maternally suppressed gene that is associated paternally with handedness and schizophrenia. Molecular Psychiatry 12(12):1129-39, 1057. [aBC, WD]

Frawley, W. (1997) Vygotsky and cognitive science: Language and the unification of the social and computational mind. Harvard University Press. [WF]

(2002a) Control in cross-domain mental computation: Evidence from language breakdown. Computational Intelligence 18:1-28. [WF]

(2002b) Inner speech and the meeting of the minds. Behavioral and Brain Sciences 25:686-87. [WF]

Freeman, D. (2007) Suspicious minds: The psychology of persecutory delusions. Clinical Psychology Review 27(4):425-57. [KNT]

Freud, S. (1905/1953) Three essays on the theory of sexuality. In: The standard edition of the complete psychological works of Sigmund Freud, vol. 7, ed. J. Strachey, A. Freud, A. Strachey \& A. Tyson, pp. 136-243. The Hogarth Press and the Institute of Psychoanalysis. [rBC]

Friedman, J. I., Vrijenhoek, T., Markx, S., Janssen, I. M., van der Vliet, W. A., Faas, B. H., Knoers, N. V., Cahn, W., Kahn, R. S., Edelmann, L., Davis, K. L., Silverman, J. M., Brunner, H. G., van Kessel, A. G., Wijmenga, C., Ophoff, R. A. \& Veltman, J. A. (2008) CNTNAP2 gene dosage variation is associated with schizophrenia and epilepsy. Molecular Psychiatry 13:261-66. [rBC]

Frith, C. D. (1992) The cognitive neuropsychology of schizophrenia. Psychology Press/ Erlbaum. [arBC, KNT]

(1996) The role of the prefrontal cortex in self-consciousness: The case of auditory hallucinations. Philosophical Transactions of the Royal Society of London, Series B: Biological Sciences 351:1505-12. [aBC]

(2004) Schizophrenia and theory of mind. Psychological Medicine 34:385-89. [aBC, AT]

(2005a) The neural basis of hallucinations and delusions. Comptes Rendus Biologies 328:169-75. [MKB]

(2005b) The self in action: Lessons from delusions of control. Consciousness and Cognition 14:752-70. [aBC]

Frith, C. D. \& Allen, H. A. (1988) Language disorders in schizophrenia and their implications for neuropsychology. In: Schizophrenia: The major issues, ed. P. Bebbington \& P. McGuffin. Heinemann. [aBC]

Frith, C. D., Blakemore, S. J. \& Wolpert, D. M. (2000) Abnormalities in the awareness and control of action. Philosophical Transactions of the Royal Society of London, Series B: Biological Sciences 355:1771-88. [aBC]

Frith, C. D. \& Frith, U. (1991) Elective affinities in schizophrenia and childhood autism. In: Social psychiatry: Theory, methodology and practice, ed. P. Bebbington, pp. 65-88. Transactions Press. [aBC]

(1999) Interacting minds - A biological basis. Science 286:1692-95. $[\mathrm{aBC}]$

Frith, U. (2003) Autism: Explaining the enigma. Blackwell. [arBC]

(2004) Emanuel Miller lecture: Confusions and controversies about Asperger syndrome. Journal of Child Psychology and Psychiatry, and Allied Disciplines 45:672-86. [aBC]

Frith, U. \& Happé, F. (2005) Autism spectrum disorder. Current Biology 15:R78690. [aBC]

Fukumoto, A., Hashimoto, T., Ito, H., Nishimura, M., Tsuda, Y., Miyazaki, M., Mori, K., Arisawa, K. \& Kagami, S. (2008) Growth of head circumference in autistic infants during the first year of life. Journal of Autism and Developmental Disorders 38:411-18. [aBC]

Fults, D. W. (2005) Modeling medulloblastoma with genetically engineered mice. Neurosurgical Focus 19:E7. [aBC]

Gabbard, G. O. (1990) Psychodynamic psychiatry in clinical practice. American Psychiatric Press. [R-PB]

Gaebel, W. \& Wölwer, W. (2004) Facial expressivity in the course of schizophrenia and depression. European Archives of Psychiatry and Clinical Neuroscience 254:335-42. [aBC]

Gagnon, R. (2003) Placental insufficiency and its consequences. European Journal of Obstetrics, Gynecology, and Reproductive Biology 110(1):S99-107. $[\mathrm{aBC}]$

Galaburda, A. M. (1984) Anatomical asymmetries. In: Cerebral dominance, ed. N Geschwind \& A. M. Galaburda, pp. 11-25. Harvard University Press. [aBC]

Gale, C. R. \& Martyn, C. N. (2004) Birthweight and later risk of depression in a national birth cohort. British Journal of Psychiatry 184:28-33. [aBC]
Gallese, V. (2006) Intentional attunement: A neurophysiological perspective on social cognition and its disruption in autism. Brain Research 1079:15-24. $[\mathrm{aBC}]$

Gardiner, J. M. (2002) Episodic memory and autonoetic consciousness: A first-person approach. In: Episodic memory: New directions in research, ed. A. Baddeley, J. P. Aggleton \& M. A. Conway. Oxford University Press. [aBC]

Gardiner, J. M., Bowler, D. M. \& Grice, S. J. (2003) Further evidence of preserved priming and impaired recall in adults with Asperger's syndrome. Journal of Autism and Developmental Disorders 33:259-69. [aBC]

Garrity, A. G., Pearlson, G. D., McKiernan, K., Lloyd, D., Kiehl, K. A. \& Calhoun, V. D. (2007) Aberrant "default mode" functional connectivity in schizophrenia. American Journal of Psychiatry 164:450-57. [R-PB, aBC]

Ge, Y., Grossman, R. I., Babb, J. S., Rabin, M. L., Mannon, L. J. \& Kolson, D. L. (2002) Age-related total gray matter and white matter changes in normal adult brain. Part I: Volumetric MR imaging analysis. American Journal of Neuroradiology 23:1327-33. [aBC]

Gernsbacher, M. A., Dawson, M. \& Mottron, L. (2006) Autism: Common, heritable, but not harmful. Behavioral and Brain Sciences 29:413-14. [aBC]

Geschwind, D. H., Boone, K. B., Miller, B. L. \& Swerdloff, R. S. (2000) Neurobehavioral phenotype of Klinefelter syndrome. Mental Retardation and Developmental Disabilities Research Reviews 6:107-16. [rBC]

Geschwind, D. H. \& Levitt, P. (2007) Autism spectrum disorders: Developmental disconnection syndromes. Current Opinion in Neurobiology 17(1):103-11. [CKD]

Geuze, E., Vermetten, E. \& Bremner, J. D. (2005) MR-based in vivo hippocampal volumetrics: 2. Findings in neuropsychiatric disorders. Molecular Psychiatry $10: 160-84 . \quad[\mathrm{aBC}]$

Gianotti, L. R., Mohr, C., Pizzagalli, D., Lehmann, D. \& Brugger, P. (2001) Associative processing and paranormal belief. Psychiatry and Clinical Neurosciences 55:595-603. [aBC]

Gibson, M. A. \& Mace, R. (2003) Strong mothers bear more sons in rural Ethiopia Proceedings of the Royal Society of London Series B: Biological Sciences 270(Suppl. 1):S108-109. [aBC]

Gillessen-Kaesbach, G., Robinson, W., Lohmann, D., Kaya-Westerloh, S Passarge, E. \& Horsthemke, B. (1995) Genotype-phenotype correlation in a series of 167 deletion and non-deletion patients with Prader-Willi syndrome. Human Genetics 96:638-43. [aBC]

Gimelbrant, A., Hutchinson, J. N., Thompson, B. R. \& Chess, A. (2007) Widespread monoallelic expression on human autosomes. Science 318:1136-40. [rBC]

Ginsburg, J. (2004) Coughs and sneezes spread mind diseases. New Scientist 184:40-43. [rBC]

Girirajan, S., Vlangos, C. N., Szomju, B. B., Edelman, E., Trevors, C. D., Dupuis, L., Nezarati, M., Bunyan, D. J. \& Elsea, S. H. (2006) Genotype-phenotype correlation in Smith-Magenis syndrome: Evidence that multiple genes in $17 \mathrm{p} 11.2$ contribute to the clinical spectrum. Genetics in Medicine 8:417-27. $[\mathrm{rBC}]$

Gisabella, B., Bolshakov, V. Y. \& Benes, F. M. (2005) Regulation of synaptic plasticity in a schizophrenia model. Proceedings of the National Academy of Sciences of the United States of America 102:13301-306. [aBC]

Gläscher, J. \& Adolphs, R. (2003) Processing of the arousal of subliminal and supraliminal emotional stimuli by the human amygdala. Journal of Neuroscience 23:10274-82. [aBC]

Glaser, R. L., Ramsay, J. P. \& Morison, I. M. (2006) The imprinted gene and parent-of-origin effect database now includes parental origin of de novo mutations. Nucleic Acids Research 34:D29-31. [rBC]

Gobrogge, K. L., Breedlove, S. M. \& Klump, K. L. (2008) Genetic and environmental influences on 2D:4D finger length ratios: A study of monozygotic and dizygotic male and female twins. Archives of Sexual Behavior 37:112-18. [MV]

Goel, V., Grafman, J., Sadato, N. \& Hallett, M. (1995) Modeling other minds. Neuroreport 6:1741-46. [aBC]

Goghari, V. M., Rehm, K., Carter, C. S. \& Macdonald, A. W., III (2007) Regionally specific cortical thinning and gray matter abnormalities in the healthy relatives of schizophrenia patients. Cerebral Cortex 17:415-24. [aBC]

Goldacre, M. J., Kurina, L. M., Wotton, C. J., Yeates, D. \& Seagroat, V. (2005) Schizophrenia and cancer: An epidemiological study. British Journal of Psychiatry 187:334-38. [aBC]

Goldstein, G., Johnson, C. R. \& Minshew, N. J. (2001a) Attentional processes in autism. Journal of Autism and Developmental Disorders 31:433-40. [aBC]

Goldstein, G., Minshew, N. J., Allen, D. N. \& Seaton, B. E. (2002) High-functioning autism and schizophrenia: A comparison of an early and late onset neurodevelopmental disorder. Archives of Clinical Neuropsychology $17: 461-75 . \quad[\mathrm{aBC}]$

Goldstein, J. M., Seidman, L. J., Horton, N. J., Makris, N., Kennedy, D. N., Caviness, V. S., Jr., Faraone, S. V. \& Tsuang, M. T. (2001b) Normal sexual dimorphism of the adult human brain assessed by in vivo magnetic resonance imaging. Cerebral Cortex 11:490-97. [aBC] 
Goldstone, A. P. (2004) Prader-Willi syndrome: Advances in genetics, pathophysiology and treatment. Trends in Endocrinology and Metabolism 15:12-20. [aBC]

Golimbet, V. E., Alfimova, M. V., Shcherbatikh, T., Kaleda, V. G., Abramova, L. I. \& Rogaev, E. I. (2003) Serotonin transporter gene polymorphism and schizoid personality traits in the patients with psychosis and psychiatrically well subjects. World Journal of Biological Psychiatry 4(1):25-29. [ST]

Gomot, M., Bernard, F. A., Davis, M. H., Belmonte, M. K., Ashwin, C., Bullmore, E. T. \& Baron-Cohen, S. (2006) Change detection in children with autism: An auditory event-related fMRI study. NeuroImage 29:475-84. [aBC]

Good, C. D., Johnsrude, I., Ashburner, J., Henson, R. N., Friston, K. J. \& Frackowiak, R. S. (2001) Cerebral asymmetry and the effects of sex and handedness on brain structure: A voxel-based morphometric analysis of 465 normal adult human brains. NeuroImage 14:685-700. [aBC]

Goos, L. M., Ezzatian, P. \& Schachar, R. (2007) Parent-of-origin effects in attention-deficit hyperactivity disorder. Psychiatry Research 149(1-3):1-9. [aBC, LMG]

Goos, L. M. \& Ragsdale, G. (2008) Genomic imprinting and human psychology: Cognition, behavior and pathology. In: Genomic imprinting, ed. J. Wilkins, pp. 71-88. Springer. [LMG]

Gorlova, O. Y., Amos, C. I., Wang, N. W., Shete, S., Turner, S. T. \& Boerwinkle, E. (2003) Genetic linkage and imprinting effects on body mass index in children and young adults. European Journal of Human Genetics 11:425-32. [aBC]

Gothelf, D. (2007) Velocardiofacial syndrome. Child and Adolescent Psychiatric Clinics of North America 16:677-93. [arBC]

Gottesman, I. \& Gould, T. D. (2003) The endophenotype concept in psychiatry: Etymology and strategic intentions. American Journal of Psychiatry 160(4):636-45. [LMG]

Goussé, V., Plumet, M. H., Chabane, N., Mouren-Siméoni, M., Ferradian, N. \& Leboyer, M. (2002) Fringe phenotypes in autism: A review of clinical, biochemical and cognitive studies. European Psychiatry 17:120-28. [aBC]

Grandin, T. (1995) Thinking in pictures and other reports from my life with autism. Vintage Books. [aBC]

(2004) Label of "autism" could hold back gifted children. Nature 430:399. $[\mathrm{aBC}]$

Grandin, T. \& Johnson, C. (2005) Animals in translation: Using the mysteries of autism to decode animal behavior. Scribner. [rBC]

Granholm, E., Perry, W., Filoteo, J. V. \& Braff, D. (1999) Hemispheric and attentional contributions to perceptual organization deficits on the global-local task in schizophrenia. Neuropsychology 13:271-81. [aBC]

Gray, J. A. (1998) Integrating schizophrenia. Schizophrenia Bulletin 24:249-66. $[\mathrm{aBC}]$

Gray, V., Karmiloff-Smith, A., Funnell, E. \& Tassabehji, M. (2006) In-depth analysis of spatial cognition in Williams syndrome: A critical assessment of the role of the LIMK1 gene. Neuropsychologia 44:679-85. [rBC]

Green, L. A., Fein, D., Modahl, C., Feinstein, C., Waterhouse, L. \& Morris, M. (2001) Oxytocin and autistic disorder: Alterations in peptide forms. Biological Psychiatry 50:609-13. [R-PB, aBC, LMG]

Green, M. J. \& Phillips, M. L. (2004) Social threat perception and the evolution of paranoia. Neuroscience and Biobehavioral Reviews 28:333-42. $[\mathrm{aBC}]$

Greicius, M. D., Flores, B. H., Menon, V., Glover, G. H., Solvason, H. B., Kenna H., Reiss, A. L. \& Schatzberg, A. F. (2007) Resting-state functional connectivity in major depression: Abnormally increased contributions from subgenual cingulate cortex and thalamus. Biological Psychiatry 62:429-37. [aBC]
[a.

Greicius, M. D., Krasnow, B., Reiss, A. L. \& Menon, V. (2003) Functional connectivity in the resting brain: A network analysis of the default mode hypothesis. Proceedings of the National Academy of Sciences of the United States of America 100:253-58. [aBC]

Grigorenko, E. L., Klin, A. \& Volkmar, F. (2003) Annotation: Hyperlexia: Disability or superability? Journal of Child Psychology and Psychiatry, and Allied Disciplines 44:1079-91. [aBC]

Griskevicius, V., Cialdini, R. B. \& Kenrick, D. T. (2006) Peacocks, Picasso, and parental investment: The effects of romantic motives on creativity. Journal of Personality and Social Psychology 91:63-76. [DN]

Gropman, A. L., Elsea, S., Duncan, W. C. \& Smith, A. C. M. (2007) New developments in Smith-Magenis syndrome (del 17p11.2). Current Opinion in Neurology 20:125-34. [rBC]

Grossberg, S. (2000a) How hallucinations may arise from brain mechanisms of learning, attention, and volition. Journal of the International Neuropsychological Society 6(5):583-92. [TV]

(2000b) The imbalanced brain: From normal behavior to schizophrenia. Biological Psychiatry 48:81-98. [aBC]

Grossberg, S. \& Seidman, D. (2006) Neural dynamics of autistic behaviors: Cognitive, emotional, and timing substrates. Neuropsychology Review 113(3):483525. [TV]

Guieu, R., Samuelian, J. C. \& Coulouvrat, H. (1994) Objective evaluation of pain perception in patients with schizophrenia. British Journal of Psychiatry 164:253-55. [ST]
Gunnell, D. \& Holly, J. M. (2004) Hypothesis: Do insulin-like growth factors underlie associations of birth complications, fetal and pre-adult growth with schizophrenia? Schizophrenia Research 71:191-93. [aBC]

Gunnell, D., Rasmussen, F., Fouskakis, D., Tynelius, P. \& Harrison, G. (2003) Patterns of fetal and childhood growth and the development of psychosis in young males: A cohort study. American Journal of Epidemiology 158:291-300. [aBC]

Gunter, H. L., Ghaziuddin, M. \& Ellis, H. D. (2002) Asperger syndrome: Tests of right hemisphere functioning and interhemispheric communication. Journal of Autism and Developmental Disorders 32:263-81. [aBC]

Gur, R. C., Alsop, D., Glahn, D., Petty, R., Swanson, C. L., Maldjian, J. A., Turetsky, B. I., Detre, J. A., Gee, J. \& Gur, R. E. (2000) An fMRI study of sex differences in regional activation to a verbal and a spatial task. Brain and Language 74:157-70. [aBC]

Gur, R. E., Keshavan, M. S. \& Lawrie, S. M. (2007) Deconstructing psychosis with human brain imaging. Schizophrenia Bulletin 33:921-31. [aBC]

Gur, R. E., Kohler, C., Turetsky, B. I., Siegel, S. J., Kanes, S. J., Bilker, W. B., Brennan, A. R. \& Gur, R. C. (2004) A sexually dimorphic ratio of orbitofrontal to amygdala volume is altered in schizophrenia. Biological Psychiatry 55:51217. $[\mathrm{aBC}]$

Gur, R. E., McGrath, C., Chan, R. M., Schroeder, L., Turner, T., Turetsky, B. I., Kohler, C., Alsop, D., Maldjian, J., Ragland, J. D. \& Gur, R. C. (2002) An fMRI study of facial emotion processing in patients with schizophrenia. American Journal of Psychiatry 159:1992-99. [aBC]

Guthrie, S. E. (1993) Faces in the clouds: A new theory of religion. Oxford University Press. [SK]

Hadjikhani, N., Joseph, R. M., Snyder, J. \& Tager-Flusberg, H. (2006) Anatomical differences in the mirror neuron system and social cognition network in autism. Cerebral Cortex 16:1276-82. [aBC]

(2007) Abnormal activation of the social brain during face perception in autism. Human Brain Mapping 28:441-49. [aBC]

Haig, D. (1993) Genetic conflicts in human pregnancy. Quarterly Review of Biology $68: 495-532 . \quad[\mathrm{aBC}]$

(1996) Placental hormones, genetic imprinting, and maternal-fetal communication. Journal of Evolutionary Biology 9:357. [aBC]

(1997) Parental antagonism, relatedness asymmetries, and genomic imprinting. Proceedings of the Royal Society of London B 264:1657-62. [DH]

(1999a) Asymmetric relations: Internal conflicts and the horror of incest. Evolution and Human Behavior 20:83-98. [DH]

(1999b) Genetic conflicts of pregnancy and childhood. In: Evolution in health and disease, ed. C. Stearns, pp. 77-90. Oxford University Press. $[\mathrm{aBC}]$

(2000a) Genomic imprinting, sex-biased dispersal, and social behavior. In: Evolutionary perspectives on human reproductive behavior, ed. D. LeCroy \& P. Moller. New York Academy of Sciences. Annals of the New York Academy of Sciences 907:149-63. [aBC, WD, LMG, DH]

(2000b) The kinship theory of genomic imprinting. Annual Review of Ecology and Systematics 31:9-32. [aBC]

(2003) On intrapersonal reciprocity. Evolution and Human Behavior 24:418-25. [aBC]

(2004a) Evolutionary conflicts in pregnancy and calcium metabolism - A review. Placenta 25(Suppl. A):S10-S15. [aBC]

(2004b) Genomic imprinting and kinship: How good is the evidence? Annual Review of Genetics 38:553-85. [aBC]

(2006) Intragenomic politics. Cytogenetic and Genome Research 113:68-74. [arBC]

Haig, D. \& Graham, C. (1991) Genomic imprinting and the strange case of the insulin-like growth factor II receptor. Cell 64:1045-46. [LMG]

Haig, D. \& Westoby, M. (1989) Parent specific gene expression and the triploid endosperm. American Naturalist 134:147-55. [BJAD, LMG]

Haig, D. \& Wharton, R. (2003) Prader-Willi syndrome and the evolution of human childhood. American Journal of Human Biology 15:320-29. [arBC]

Halbreich, U. \& Kahn, L. S. (2003) Hormonal aspects of schizophrenias: An overview. Psychoneuroendocrinology 28(2):1-16. [aBC]

Hallet, M. (2007) Transcranial magnetic stimulation: A primer. Neuron 55:187-99. [LMO]

Hamer, D. (2004) The god gene: How faith is hardwired into our genes. Anchor Books. [rBC]

Hamilton, A. F. de C., Brindley, R. M. \& Frith, U. (2007) Imitation and action understanding in autistic spectrum disorders: How valid is the hypothesis of a deficit in the mirror neuron system? Neuropsychologia 45:1859-68. $[\mathrm{aBC}]$

Hamilton, W. D. (1964) The genetical evolution of social behavior. Journal of Theoretical Biology 7:1-52. [aBC]

(ed.) (1996) Narrow roads of gene land, vol. 1: Evolution of social behaviour. Spektrum. [rBC]

(2005) Narrow roads of gene land, vol. 3: Last words, ed. M. Ridley. Oxford University Press. [aBC] 
Han, S., Weaver, J. A., Murray, S. O., Kang, X., Yund, E. W. \& Woods, D. L. (2002) Hemispheric asymmetry in global/local processing: Effects of stimulus position and spatial frequency. NeuroImage 17:1290-99. [aBC]

Happé, F. (1994) Autism: An introduction to psychological theory. UCL Press. $[\mathrm{aBC}]$

Happé, F., Ehlers, S., Fletcher, P., Frith, U., Johansson, M., Gillberg, C., Dolan, R., Frackowiak, R. \& Frith, C. (1996) "Theory of mind" in the brain. Evidence from a PET scan study of Asperger syndrome. Neuroreport 8:197-201. $[\mathrm{aBC}]$

Happé, F. \& Frith, U. (2006) The weak coherence account: Detail-focused cognitive style in autism spectrum disorders. Journal of Autism and Developmental Disorders 36:5-25. [aBC]

Happé, F., Ronald, A. \& Plomin, R. (2006) Time to give up on a single explanation for autism. Nature Neuroscience 9(10):1218-20. [arBC, BJAD, GV]

Hardan, A. Y., Muddasani, S., Vemulapalli, M., Keshavan, M. S. \& Minshew, N. J. (2006) An MRI study of increased cortical thickness in autism. American Journal of Psychiatry 163:1290-92. [aBC]

Hardy-Baylé, M. C. (1994) Organisation de l'action, phénomènes de conscience et représentation mentale de l'action chez des schizophrènes [Organization of action; the phenomenon of consciousness and mental representation of action in schizophrenia]. Actualités Psychiatriques 20:393-400. [KNT]

Hardy-Baylé, M. C., Sarfati, Y. \& Passerieux, C. (2003) The cognitive basis of disorganization symptomatology in schizophrenia and its clinical correlates: Toward a pathogenetic approach to disorganization. Schizophrenia Bulletin 29:459-71. [KNT]

Harrington, L., Langdon, R., Siegert, R. J. \& McClure, J. (2005a) Schizophrenia, theory of mind, and persecutory delusions. Cognitive Neuropsychiatry 10:87-104. [arBC, SvR]

Harrington, L., Siegert, R. J. \& McClure, J. (2005b) Theory of mind in schizophrenia: A critical review. Cognitive Neuropsychiatry 10:249-86. [aBC, KNT]

Harris, N. S., Courchesne, E., Townsend, J., Carper, R. A. \& Lord, C. (1999) Neuroanatomic contributions to slowed orienting of attention in children with autism. Brain Research Cognitive Brain Research 8:61-71. [R-PB]

Harrison, B. J., Yücel, M., Pujol, J. \& Pantelis, C. (2007) Task-induced deactivation of midline cortical regions in schizophrenia assessed with fMRI. Schizophrenia Research 91:82-86. [aBC]

Harrison, P. J. (1999) The neuropathology of schizophrenia. A critical review of the data and their interpretation. Brain 122(Pt 4):593-624. [aBC]

Hartmann, W., Koch, A., Brune, H., Waha, A., Schuller, U., Dani, I., Denkhaus, D., Langmann, W., Bode, U., Wiestler, O. D., Schilling, K. \& Pietsch, T. (2005) Insulin-like growth factor II is involved in the proliferation control of medulloblastoma and its cerebellar precursor cells. American Journal of Pathology 166:1153-62. [aBC]

Haselton, M. G. \& Miller, G. F. (2006) Women's fertility across the cycle increases the short-term attractiveness of creative intelligence. Human Nature 17:50-73. [DN]

Haselton, M. G. \& Nettle, D. (2006) The paranoid optimist: An integrative evolutionary model of cognitive biases. Personality and Social Psychology Review 10:47-66. [SK]

Hashimoto, T., Bergen, S. E., Nguyen, Q. L., Xu, B., Monteggia, L. M., Pierri, J. N., Sun, Z., Sampson, A. R. \& Lewis, D. A. (2005) Relationship of brainderived neurotrophic factor and its receptor TrkB to altered inhibitory prefrontal circuitry in schizophrenia. Journal of Neuroscience 25:372-83. $[\mathrm{aBC}]$

Haskins, B. G. \& Silva, J. A. (2006) Asperger's disorder and criminal behavior: Forensic-psychiatric considerations. Journal of the American Academy of Psychiatry and the Law 34:374-84. [aBC]

Hassed, S., Vaz, S. A., Lee, J., Mulvihill, J. J. \& Li, S. (2004) Expanded phenotype of the 22q duplication syndrome. American Journal of Human Genetics 75(Suppl.):151. [rBC]

Hattori, J., Ogino, T., Abiru, K., Nakano, K., Oka, M. \& Ohtsuka, Y. (2006) Are pervasive developmental disorders and attention-deficit/hyperactivity disorder distinct disorders? Brain and Development 28:371-74. [aBC]

Haukka, J., Suvisaari, J., Hakkinen, L. \& Lönnqvist, J. (2008) Growth pattern and risk of schizophrenia. Psychological Medicine 38:63-70. [aBC]

Haukka, J., Suvisaari, J. \& Lönnqvist, J. (2003) Fertility of patients with schizophrenia, their siblings, and the general population: A cohort study from 1950 to 1959 in Finland. American Journal of Psychiatry 160:460-63. [arBC, MCK]

Havlovicova, M., Novotna, D, Kocarek, E, Novotna, K, Bendova, S, Petrak, B, Hrdlicka, M., \& Sedlacek, Z. (2007) A girl with Neurofibromatosis type 1, atypical autism and mosaic ring chromosome 17. American Journal of Medical Genetics 143A:76-81. [CKD]

Hayashi, M., Kato, M., Igarashi, K. \& Kashima, H. (2008) Superior fluid intelligence in children with Asperger's disorder. Brain and Cognition 66:306-10. [rBC]

Hayesmoore, J. B., Bray, N. J., Owen, M. J. \& O’Donovan, M. C. (in press) DISC1 mRNA expression is not influenced by common Cis-acting regulatory polymorphisms or imprinting. American Journal of Medical Genetics, Part B: Neuropsychiatric Genetics. [rBC]

Heaton, P. \& Wallace, G. L. (2004) Annotation: The savant syndrome. Journal of Child Psychology and Psychiatry, and Allied Disciplines 45:899-911. [aBC]

Heerey, E. A., Keltner, D. \& Capps, L. M. (2003) Making sense of self-conscious emotion: Linking theory of mind and emotion in children with autism. Emotion 3:394-400. [aBC]

Heim, S., Kissler, J., Elbert, T. \& Rockstroh, B. (2004) Cerebral lateralization in schizophrenia and dyslexia: Neuromagnetic responses to auditory stimuli. Neuropsychologia 42:692-97. [aBC]

Herbert, M. R. (2005) Large brains in autism: The challenge of pervasive abnormality. Neuroscientist 11:417-40. [aBC]

Herbert, M. R., Harris, G. J., Adrien, K. T., Ziegler, D. A., Makris, N., Kennedy, D. N., Lange, N. T., Chabris, C. F., Bakardjiev, A., Hodgson, J., Takeoka, M., Tager-Flusberg, H. \& Caviness, V. S., Jr. (2002) Abnormal asymmetry in language association cortex in autism. Annals of Neurology 52:588-96. [aBC]

Herbert, M. R. \& Kenet, T. (2007) Brain abnormalities in language disorders and in autism. Pediatric Clinics of North America 54:563-83. [aBC]

Herbert, M. R., Ziegler, D. A., Deutsch, C. K., O'Brien, L. M., Kennedy, D. N., Filipek, P. A., Bakardjiev, A. I., Hodgson, J., Takeoka, M., Makris, N. \& Caviness, V. S., Jr. (2005) Brain asymmetries in autism and developmental language disorder: A nested whole-brain analysis. Brain 128:213-26. [aBC]

Herbert, M. R., Ziegler, D. A., Makris, N., Filipek, P. A., Kemper, T. L., Normandin, J. J., Sanders, H. A., Kennedy, D. N. \& Caviness, V. S., Jr. (2004) Localization of white matter volume increase in autism and developmental language disorder. Annals of Neurology 55:530-40. [aBC]

Hermelin, B. (2001) Bright splinters of the mind: A personal story of research with autistic savants. Jessica Kingsley. $\quad[\mathrm{rBC}]$

Herrera, E. (2002) Implications of dietary fatty acids during pregnancy on placental, fetal and postnatal development - A review. Placenta 23(Suppl. A):S9-S19. $[\mathrm{aBC}]$

Highley, J. R., DeLisi, L. E., Roberts, N., Webb, J. A., Relja, M., Razi, K. \& Crow, T. J. (2003) Sex-dependent effects of schizophrenia: An MRI study of gyral folding, and cortical and white matter volume. Psychiatry Research 124:11-23. [aBC]

Hill, E. L. \& Frith, U. (2003) Understanding autism: Insights from mind and brain. Philosophical Transactions of the Royal Society of London. Series B, Biological Sciences 358:281-89. [aBC]

Hobbs, K., Kennedy, A., Dubray, M., Bigler, E. D., Petersen, P. B., McMahon, W. \& Lainhart, J. E. (2007) A retrospective fetal ultrasound study of brain size in autism. Biological Psychiatry 62:1048-55. [rBC]

Hoffman, R. E., Hampson, M., Varanko, M. \& McGlashan, T. H. (2004) Auditory hallucinations, network connectivity, and schizophrenia. Behavioral and Brain Sciences 27:860-61. [aBC]

Holland, A., Whittington, J. \& Hinton, E. (2003) The paradox of Prader-Willi syndrome: A genetic model of starvation. Lancet 362:989-91. [aBC]

Hollander, E., Novotny, S., Hanratty, M., Yaffe, R., DeCaria, C. M., Aronowitz, B. R. \& Mosovich, S. (2003) Oxytocin infusion reduces repetitive behaviors in adults with autistic and Asperger's disorders. Neuropsychopharmacology 28(1):193-98. [LMG]

Holm, V. A., Cassidy, S. B., Butler, M. G., Hanchett, J. M., Greenswag, L. R., Whitman, B. Y. \& Greenberg, F. (1993) Prader-Willi syndrome: Consensus diagnostic criteria. Pediatrics 91:398-402. [aBC]

Holsen, L. \& Thompson, T. (2004) Compulsive behavior and eye blink in PraderWilli syndrome: Neurochemical implications. American Journal of Mental Retardation 109:197-207. [aBC]

Holt, D. J., Kunkel, L., Weiss, A. P., Goff, D. C., Wright, C. I., Shin, L. M., Rauch, S. L., Hootnick, J. \& Heckers, S. (2006) Increased medial temporal lobe activation during the passive viewing of emotional and neutral facial expressions in schizophrenia. Schizophrenia Research 82:153-62. $[\mathrm{aBC}]$

Holtmann, M., Bölte, S. \& Poustka, F. (2007) Autism spectrum disorders: Sex differences in autistic behaviour domains and coexisting psychopathology. Developmental Medicine and Child Neurology 49:361-66. [aBC]

Honea, R., Crow, T. J., Passingham, D. \& Mackay, C. E. (2005) Regional deficits in brain volume in schizophrenia: A meta-analysis of voxel-based morphometry studies. American Journal of Psychiatry 162:2233-45. [aBC]

Honey, E., Leekam, S. R., Turner, M. \& McConachie, H. (2006) Repetitive behaviour and play in typically developing children and children with autism. Journal of Autism and Developmental Disorders 37:1107-15. [aBC]

Hooker, C. \& Park, S. (2005) You must be looking at me: The nature of gaze perception in schizophrenia patients. Cognitive Neuropsychiatry $10: 327-45 . \quad[\mathrm{aBC}]$

Hornig, M., Weissenbock, H., Horscroft, N. \& Lipkin, W. I. (1999) An infectionbased model of neurodevelopmental damage. Proceedings of the National Academy of Sciences USA 96(21):12102-107. [ТHJB] 
Houston, R. \& Frith, U. (2000) Autism in history: The case of Hugh Blair of Borgue. Blackwell. [rBC]

Howlin, P., Baron-Cohen, S. \& Hadwin, J. (1999) Teaching children with autism to mind-read: A practical guide. Wiley. [rBC]

Hrdy, S. B. (1999) Mother nature: A history of mothers, infants, and natural selection. Pantheon. [aBC]

Hubl, D., Koenig, T., Strik, W., Federspiel, A., Kreis, R., Boesch, C., Maier, S. E., Schroth, G., Lovblad, K. \& Dierks, T. (2004) Pathways that make voices: White matter changes in auditory hallucinations. Archives of General Psychiatry 61:658-68. $\quad[\mathrm{aBC}]$

Hulshoff Pol, H. E., Schnack, H. G., Mandl, R. C. W., Brans, R. G. H., van Haren, N. E., Neeltje, E. M., Baaré, W. F. C., van Oel, C. J., Collins, D. L., Evans, A. C. \& Kahn, R. S. (2006) Gray and white matter density changes in monozygotic and same-sex dizygotic twins discordant for schizophrenia using voxel-based morphometry. NeuroImage 31:482-88. [aBC]

Humphrey, N. K. (1976) The social function of intellect. In: Growing points in ethology, ed. P. P. G. Bateson \& R. A. Hinde, pp. 303-17. Cambridge University Press. [aBC]

(1983) Consciousness regained. Oxford University Press. [aBC]

(1996) Leaps of faith: Science, miracles, and the search for supernatural consolation. Springer-Verlag. $\quad[\mathrm{rBC}]$

Hurlburt, R. T., Happé, F. \& Frith, U. (1994) Sampling the form of inner experience in three adults with Asperger syndrome. Psychological Medicine 24:385-95. [aBC]

Hutchinson, G., Bhugra, D., Mallett, R., Burnett, R., Corridan, B. \& Leff, J. (1999) Fertility and marital rates in first-onset schizophrenia. Social Psychiatry and Psychiatric Epidemiology 34:617-21. [aBC]

Huxley, J., Mayr, E., Osmond, H. \& Hoffer, A. (1964) Schizophrenia as a genetic morphism. Nature 204:220-21. [aBC]

Iacoboni, M. \& Dapretto, M. (2006) The mirror neuron system and the consequences of its dysfunction. Nature Reviews Neuroscience 7:942-51. [aBC]

Iafrate, A. J., Feuk, L., Rivera, M. N., Listewnik, M. L., Donahoe, P. K., Qi, Y., Scherer, S. W. \& Lee, C. (2004) Detection of large-scale variation in the human genome. Nature Genetics 36(9):949-51. [CKD]

Ingudomnukul, E., Baron-Cohen, S., Wheelwright, S. \& Knickmeyer, R. (2007) Elevated rates of testosterone-related disorders in women with autism spectrum conditions. Hormones and Behavior 51:597-604. [aBC]

Irwin, H. J. (1993) Belief in the paranormal: A review of the empirical literature. Journal of the American Society for Psychical Research 87:1-39. [rBC]

Isanon, A. (2001) Spirituality and the autism spectrum. Jessica Kingsley. [rBC]

Isles, A. R., Davies, W. \& Wilkinson, L. S. (2006) Genomic imprinting and the social brain. Philosophical Transactions of the Royal Society of London. Series B, Biological Sciences 361(1476):2229-37. [aBC, WD]

Isles, A. R. \& Holland, A. J. (2005) Imprinted genes and mother-offspring interactions. Early Human Development 81(1):73-77. [WD]

Izard, C. (1994) Innate and universal facial expressions: Evidence from developmental and cross-cultural research. Psychological Bulletin 115:288-99. [R-PB]

Jablensky, A., Sartorius, N., Ernberg, G., Anker, M., Korten, A., Cooper, J. E., Day, R. \& Bertelsen, A. (1992) Schizophrenia: Manifestations, incidence and course in different cultures. A World Health Organization ten-country study. Psychological Medicine Monographs 20:1-97. [MCK]

Jackson, M. (1997) Benign schizotypy? The case of spiritual experience. In: Schizotypy: Implications for illness and health, ed. G. Claridge. Oxford University Press. [aBC]

Jacob, S., Brune, C. W., Carter, C. S., Leventhal, B. L., Lord, C. \& Cook, E. H., Jr. (2007) Association of the oxytocin receptor gene (OXTR) in Caucasian children and adolescents with autism. Neuroscience Letters 417(1):6-9. [LMG]

Jacobsen, L. K. \& Rapoport, J. L. (1998) Research update: Childhood-onset schizophrenia: Implications of clinical and neurobiological research. Journal of Child Psychology and Psychiatry, and Allied Disciplines 39:101-13. [rBC]

Jacobson, J. W. \& Ackerman, L. J. (1990) Differences in adaptive functioning among people with autism or mental retardation. Journal of Autism and Developmental Disorders 20:205-19. [ST]

Jahshan, C. S. \& Sergi, M. J. (2007) Theory of mind, neurocognition, and functional status in schizotypy. Schizophrenia Research 89:278-86. [aBC]

James, A. C., Crow, T. J., Renowden, S., Wardell, A. M., Smith, D. M. \& Anslow, P. (1999) Is the course of brain development in schizophrenia delayed? Evidence from onsets in adolescence. Schizophrenia Research 40:1-10. [aBC]

James, I. (2003) Singular scientists. Journal of the Royal Society of Medicine 96(1):36-39. [GV]

Jamison, K. R. (1993) Touched with fire: Manic-depressive illness and the artistic temperament. Free Press. [rBC]

Jansen, L. M. C. (1998) Blunted cortisol response to a psychosocial stressor in schizophrenia. Schizophrenia Research 33:87-94. [ST]

Jansen, L. M. C., Gispen-De Wied, C. C., Van der Gaag, R. J., Ten Hove, F., Willemsenswinkels, S. W. M., Harteveld, E. \& van Engeland, H. (2000) Unresponsiveness to psychosocial stress in a subgroup of autistic-like children, multiple complex developmental disorder. Psychoneuroendocrinology 25:753-64. [ST]

Jansen, L. M. C., Gispen-de Wied, C. C., van der Gaag, R. J. \& van Engeland, H. (2003) Differentiation between autism and multiple complex developmental disorder in response to psychosocial stress. Neuropsychopharmacology 28(3):582-90. [LMG]

Janssen, I., Krabbendam, L., Jolles, J. \& van Os, J. (2003) Alterations in theory of mind in patients with schizophrenia and non-psychotic relatives. Acta Psychiatrica Scandinavica 108:110-17. [aBC]

Jarrold, C., Butler, D. W., Cottington, E. M. \& Jimenez, F. (2000) Linking theory of mind and central coherence bias in autism and in the general population. Developmental Psychology 36:126-38. [MKB, aBC]

Järvinen-Pasley, A., Bellugi, U., Reilly, J., Mills, D. L., Galaburda, A., Reiss, A. L. \& Korenberg, J. R. (2008) Defining the social phenotype in Williams syndrome: A model for linking gene, the brain, and behavior. Development and Psychopathology 20:1-35. [rBC]

Jaspers-Fayer, F. \& Peters, M. (2005) Hand preference, magical thinking and leftright confusion. Laterality 10:183-91. [aBC]

Jedele, K. B. (2007) The overlapping spectrum of Rett and Angelman syndromes: A clinical review. Seminars in Pediatric Neurology 14:108-17. [rBC]

Jha, P., Sheth, D. \& Ghaziuddin, M. (2007) Autism spectrum disorder and Klinefelter syndrome. European Child and Adolescent Psychiatry 16:305-308. $[\mathrm{aBC}]$

Jiang, Y. H., Sahoo, T., Michaelis, R. C., Bercovich, D., Bressler, J., Kashork, C. D., Liu, Q., Shaffer, L. G., Schroer, R. J., Stockton, D. W., Spielman, R. S., Stevenson, R. E. \& Beaudet, A. L. (2004) A mixed epigenetic/genetic model for oligogenic inheritance of autism with a limited role for UBE3A. American Journal of Medical Genetics. Part A. 131:1-10. [aBC]

Jirtle, R. L. \& Skinner, M. K. (2007) Environmental epigenomics and disease susceptibility. Nature Reviews. Genetics 8(4):253-62. [GV]

Johansson, M., Wentz, E., Fernell, E., Stromland, K., Miller, M. \& Gillberg, C. (2001) Autistic spectrum disorders in Mobius sequence: A comprehensive study of 25 individuals. Developmental Medicine and Child Neurology 43(5):338-45. [CKD]

Johnson, J. D. (2005) Dysfunction of the anterior hippocampus: The cause of fundamental schizophrenic symptoms? Medical Hypotheses 65:55-60. [aBC]

Johnson, M. H. (2001) Functional brain development in humans. Nature Reviews Neuroscience 2:475-83. [R-PB]

(2005) Subcortical face processing. Nature Reviews Neuroscience 6:766-74. $[\mathrm{aBC}]$

Johnson, M. H., Dziurawiec, S., Ellis, H. \& Morton, J. (1991) Newborn's preferential tracking of face-like stimuli and its subsequent decline. Cognition 40:1-19. [R-PB]

Johnson, M. H., Halit, H., Grice, S. J. \& Karmiloff-Smith, A. (2002) Neuroimaging of typical and atypical development: A perspective from multiple levels of analysis. Development and Psychopathology 14:521-36. [MKB]

Johnson, S. C. (2003) Detecting agents. Philosophical Transactions of the Royal Society of London. Series B, Biological Sciences 358:549-59. [aBC]

Johnson, W. \& Bouchard, T. J., Jr. (2007) Sex differences in mental abilities: $\mathrm{G}$ masks the dimensions on which they lie. Intelligence 35:23-39. [aBC]

Johnstone, K. A., DuBose, A. J., Futtner, C. R., Elmore, M. D., Brannan, C. I. \& Resnick, J. L. (2006) A human imprinting centre demonstrates conserved acquisition but diverged maintenance of imprinting in a mouse model for Angelman syndrome imprinting defects. Human Molecular Genetics 15:393-404. [arBC]

Jolly, A. (1966) Lemur behaviour. University of Chicago Press. [aBC]

Jones, S. R. \& Fernyhough, C. (2007) Thought as action: Inner speech, selfmonitoring, and auditory verbal hallucinations. Consciousness and Cognition 16:391-99. [arBC, WF]

Josse, G. \& Tzourio-Mazoyer, N. (2004) Hemispheric specialization for language. Brain Research: Brain Research Reviews 44:1-12. [aBC]

Just, M. A., Cherkassky, V. L., Keller, T. A. \& Minshew, N. J. (2004) Cortical activation and synchronization during sentence comprehension in high-functioning autism: Evidence of underconnectivity. Brain 127:1811-21. [aBC, CKD]

Juul, A., Dalgaard, P., Blum, W. F., Bang, P., Hall, K., Michaelsen, K. F., Muller, J. \& Skakkebaek, N. E. (1995) Serum levels of insulin-like growth factor (IGF)binding protein-3 (IGFBP-3) in healthy infants, children, and adolescents: The relation to IGF-I, IGF-II, IGFBP-1, IGFBP-2, age, sex, body mass index, and pubertal maturation. Journal of Clinical Endocrinology and Metabolism 80:2534-42. [MCK]

Juul-Dam, N., Townsend, J. \& Courchesne, E. (2001) Prenatal, perinatal, and neonatal factors in autism, pervasive developmental disorder-not otherwise specified, and the general population. Pediatrics 107:E63. [aBC]

Kalkman, H. O. (2006) The role of the phosphatidylinositide 3-kinase-protein kinase B pathway in schizophrenia. Pharmacology and Therapeutics 110: 117-34. [aBC] 
Kana, R. K., Keller, T. A., Cherkassky, V. L., Minshew, N. J. \& Just, M. A. (2006 Sentence comprehension in autism: Thinking in pictures with decreased functional connectivity. Brain 129:2484-93. [aBC]

Kanazawa, S. (2005) Big and tall parents have more sons: Further generalizations of the Trivers-Willard hypothesis. Journal of Theoretical Biology 235:583-90. [SK]

(2006) Violent men have more sons: Further evidence for the generalized Trivers-Willard hypothesis (gTWH). Journal of Theoretical Biology 239: 450-59. [SK]

(2007a) Beautiful parents have more daughters: A further implication of the generalized Trivers-Willard hypothesis (gTWH). Journal of Theoretical Biology 244:133-40. [SK]

(2007b) Big and tall soldiers are more likely to survive battle: A possible explanation for the "returning soldier effect" on the secondary sex ratio. Human Reproduction 22:3002-3008. [SK]

Kanazawa, S. \& Vandermassen, G. (2005) Engineers have more sons, nurses have more daughters: An evolutionary psychological extension of Baron-Cohen's extreme male brain theory of autism and its empirical implications. Journal of Theoretical Biology 233:589-99. [SK]

Kanner, L. (1943) Autistic disturbances of affective contact. Nervous Child 32: 217-53. [ST]

(1949) Problems of nosology and psychodynamics of early infantile autism. American Journal of Orthopsychiatry 19:416-26. [aBC]

(1965) Infantile autism and the schizophrenias. Behavioral Science 10:412-20. $[\mathrm{aBC}]$

Karmiloff-Smith, A. (1997) Crucial differences between developmental cognitive neuroscience and adult neuropsychology. Developmental Neuropsychology 13(4):513-24. [SvR]

(2007) Atypical epigenesis. Developmental Science 10:84-88. [MKB]

Kasai, K., Shenton, M. E., Salisbury, D. F., Hiravasu, Y., Lee, C., Ciszewski, A. A., Yurgelun-Todd, D., Kikinis, R., Jolesz, F. A. \& McCarley, R. W. (2003a) Progressive decrease of left superior temporal gyrus gray matter volume in patients with first-episode schizophrenia. American Journal of Psychiatry 160:156-64. [aBC]

Kasai, K., Shenton, M. E., Salisbury, D. F., Hirayasu, Y., Onitsuka, T., Spencer, M. H., Yurgelun-Todd, D. A., Kikinis, R., Jolesz, F. A. \& McCarley, R. W. (2003b) Progressive decrease of left Heschl gyrus and planum temporale gray matter volume in first-episode schizophrenia: A longitudinal magnetic resonance imaging study. Archives of General Psychiatry 60:766-75. [aBC]

Kayahan, B., Ozturk, O., Veznedaroglu, B. \& Eraslan, D. (2005) Obsessivecompulsive symptoms in schizophrenia: Prevalence and clinical correlates. Psychiatry and Clinical Neurosciences 59:291-95. [aBC]

Kelemen, O., Kéri, S., Must, A., Benedek, G. \& Janka, Z. (2004) No evidence for impaired "theory of mind" in unaffected first-degree relatives of schizophrenia patients. Acta Psychiatrica Scandinavica 110:146-49. [aBC]

Keller, J., Schatzberg, A. F. \& Maj, M. (2007) Current issues in the classification of psychotic major depression. Schizophrenia Research 33:877-85. [aBC]

Keller, M. C. \& Miller, G. (2006) Resolving the paradox of common, harmful, heritable mental disorders: Which evolutionary genetic models work best? Behavioral and Brain Sciences 29(4):385-452. [THJB, rBC, MCK]

Kempf, L., Hussain, N. \& Potash, J. B. (2005) Mood disorder with psychotic features, schizoaffective disorder, and schizophrenia with mood features: Trouble at the borders. International Review of Psychiatry 17:9-19. [aBC]

Kennedy, D. P., Redcay, E. \& Courchesne, E. (2006) Failing to deactivate: Resting functional abnormalities in autism. Proceedings of the National Academy of Sciences of the United States of America 103:8275-80. [aBC, R-PB]

Kennedy, G. E. (2005) From the ape's dilemma to the weanling's dilemma: Early weaning and its evolutionary context. Journal of Human Evolution 48:123-45. [DH]

Kennedy, N., McDonough, M., Kelly, B. \& Berrios, G. E. (2002) Erotomania revisited: Clinical course and treatment. Comprehensive Psychiatry 43:1-6. $[\mathrm{aBC}]$

Kent, L., Bowdin, S., Kirby, G. A., Cooper, W. N. \& Maher, E. R. (in press) Beckwith-Wiedemann syndrome: A behavioral phenotype-genotype study. American Journal of Medical Genetics, Part B: Neuropsychiatric Genetics. $[\mathrm{rBC}]$

Kerns, J. G. (2005) Positive schizotypy and emotion processing. Journal of Abnormal Psychology 114:392-401. [aBC]

Kesby, J. P., Burne, T. H., McGrath, J. J. \& Eyles, D. W. (2006) Developmental vitamin D deficiency alters MK 801-induced hyperlocomotion in the adult rat: An animal model of schizophrenia. Biological Psychiatry 60(6):591-96. [THJB]

Kesler, S. R. (2007) Turner syndrome. Child and Adolescent Psychiatric Clinics of North America 16:709-22. [rBC]

Keverne, E. B. (1999) GABA-ergic neurons and the neurobiology of schizophrenia and other psychoses. Brain Research Bulletin 48:467-73. [aBC] (2001a) Genomic imprinting and the maternal brain. Progress in Brain Research 133:279-85. [aBC]

(2001b) Genomic imprinting, maternal care, and brain evolution. Hormones and Behavior 40:146-55. [aBC]

Keverne, E. B., Fundele, R., Narasimha, M., Barton, S. C. \& Surani, M. A. (1996) Genomic imprinting and the differential roles of parental genomes in brain development. Brain Research, Developmental Brain Research 92:91-100. [rBC, BJAD, LMG]

Kieseppä, T., van Erp, T. G., Haukka, J., Partonen, T., Cannon, T. D., Poutanen, V. P., Kaprio, J. \& Lönnqvist, J. (2003) Reduced left hemispheric white matter volume in twins with bipolar I disorder. Biological Psychiatry 54:896-905. $[\mathrm{aBC}]$

Kilpinen, H., Ylisaukko-Oja, T., Hennah, W., Palo, O. M., Varilo, T., Vanhala, R., Nieminen-von Wendt, T., von Wendt, L., Paunio, T. \& Peltonen, L. (2008) Association of DISCl with autism and Asperger syndrome. Molecular Psychiatry 13(2):187-96. [rBC, GV]

Kimhy, D., Goetz, R., Yale, S., Corcoran, C. \& Malaspina, D. (2005) Delusions in individuals with schizophrenia: Factor structure, clinical correlates, and putative neurobiology. Psychopathology 38:338-44. [aBC]

Kington, J. M., Jones, L. A., Watt, A. A., Hopkin, E. J. \& Williams, J. (2000) Impaired eye expression recognition in schizophrenia. Journal of Psychiatric Research 34:341-47. [aBC]

Kirkpatrick, L. A. (2005) Attachment, evolution, and the psychology of religion. Guilford. [SK]

Kirsch, P., Esslinger, C., Chen, Q., Mier, D., Lis, S., Siddhanti, S., Gruppe, H., Mattay, V. S., Gallhofer, B. \& Meyer-Lindenberg, A. (2005) Oxytocin modulates neural circuitry for social cognition and fear in humans. Journal of Neuroscience 25:11489-93. [aBC, LMG]

Kleim, J. A., Chan, S., Pringle, K. S., Procaccio, V., Jimenez, R. \& Cramer, S. C. (2006) BDNF val66met polymorphism is associated with modified experiencedependent plasticity in human motor cortex. Nature Neuroscience 9: 735-37. [LMO]

Klejbor, I., Myers, J. M., Hausknecht, K., Corso, T. D., Gambino, A. S., Morys, J., Maher, P. A., Hard, R., Richards, J., Stachowiak, E. K. \& Stachowiak, M. K. (2006) Fibroblast growth factor receptor signaling affects development and function of dopamine neurons - Inhibition results in a schizophrenia-like syndrome in transgenic mice. Journal of Neurochemistry 97:1243-58. [aBC]

Klin, A. \& Jones, W. (2006) Attributing social and physical meaning to ambiguous visual displays in individuals with higher-functioning autism spectrum disorders. Brain and Cognition 61:40-53. [RL]

Klinger, L. G. \& Dawson, G. (2001) Prototype formation in autism. Development and Psychopathology 13(1):111-24. [TV]

Knickmeyer, R. C., Baron-Cohen, S., Raggatt, P. \& Taylor, K. (2005) Foetal testosterone, social relationships, and restricted interests in children. Journal of Child Psychology and Psychiatry, and Allied Disciplines 46:198-210. [aBC]

Knickmeyer, R. C., Wheelwright, S., Hoekstra, R. \& Baron-Cohen, S. (2006) Age of menarche in females with autism spectrum conditions. Developmental Medicine and Child Neurology 48:1007-1008. [MV]

Knoch, D., Pascual-Leone, A., Meyer, K., Treyer, V. \& Fehr, E. (2006) Diminishing reciprocal fairness by disrupting the right prefrontal cortex. Science 314: 829-32. [aBC]

Kolevzon, A., Gross, R. \& Reichenberg, A. (2007) Prenatal and perinatal risk factors for autism: A review and integration of findings. Archives of Pediatrics and Adolescent Medicine 161:326-33. [aBC]

Kolvin, I. (1971) Studies in the childhood psychoses. I. Diagnostic criteria and classification. British Journal of Psychiatry 118:381-84. [aBC]

Konstantareas, M. M. \& Hewitt, T. (2001) Autistic disorder and schizophrenia: Diagnostic overlaps. Journal of Autism and Developmental Disorders 31(1):19-28. [aBC, ST]

Kovalev, V. A., Kruggel, F. \& von Cramon, D. Y. (2003) Gender and age effects in structural brain asymmetry as measured by MRI texture analysis. NeuroImage 19:895-905. [aBC]

Kowalski, R. (2005a) How to be artificially intelligent. Available at: http://www.doc.ic.ac.uk/ rak. [WF]

(2005b) Logic and modules. Department of Computing, Imperial College London. $[\mathrm{WF}]$

Kramer, J. H., Ellenberg, L., Leonard, J. \& Share, L. J. (1996) Developmental sex differences in global-local perceptual bias. Neuropsychology 10:402-407. $[\mathrm{aBC}]$

Krauss, H., Marwinski, K., Schulze, T., Mueller, D. J., Held, T., Rietschel, M., Maier, W. \& Freyberger, H. J. (2000) Reliability and validity of the German version of the Premorbid Adjustment Scale (PAS). Nervenarzt 71(3): 188-94. [ST]

Kravariti, E., Toulopoulou, T., Mapua-Filbey, F., Schulze, K., Walshe, M., Sham, P., Murray, R. M. \& McDonald, C. (2006) Intellectual asymmetry and genetic liability in first-degree relatives of probands with schizophrenia. British Journal of Psychiatry 188:186-87. [arBC] 
Krebs, J. R. \& Davies, N. B., eds. (1991) Behavioural ecology: An evolutionary approach. Blackwell Press. [aBC]

Kremen, W. S., Faraone, S. V., Toomey, R., Seidman, L. J. \& Tsuang, M. T. (1998) Sex differences in self-reported schizotypal traits in relatives of schizophrenic probands. Schizophrenia Research 34:27-37. [aBC]

Kruglyak, L. \& Lander, E. S. (1995) High-resolution genetic mapping of complex traits. American Journal of Human Genetics 56(5):1212-23. [LMG]

Kucharska-Pietura, K., Russell, T. \& Masiak, M. (2003) Perception of negative affect in schizophrenia - functional and structural changes in the amygdala. Annales Universitatis Mariae Curie-Sklodowska. Sectio D: Medicina 58: $453-58 . \quad[\mathrm{aBC}]$

Kuhlmeier, V. A., Bloom, P. \& Wynn, K. (2004) Do 5-month-old infants see humans as material objects? Cognition $94: 95-103 . \quad$ [aBC]

Kuperberg, G. R., Broome, M. R., McGuire, P. K., David, A. S., Eddy, M., Ozawa, F., Goff, D., West, W. C., Williams, S. C. R., van der Kouwe, A. J. W., Salat, D. H., Dale, A. M. \& Fischl, B. (2003) Regionally localized thinning of the cerebral cortex in schizophrenia. Archives of General Psychiatry 60:878-88. $[\mathrm{aBC}]$

Kurita, M., Kuwajima, T., Nishimura, I. \& Yoshikawa, K. (2006) Necdin downregulates CDC2 expression to attenuate neuronal apoptosis. Journal of Neuroscience 26:12003-13. [aBC]

Kuroki, N., Kubicki, M., Nestor, P. G., Salisbury, D. F., Park, H. J., Levitt, J. J., Woolston, S., Frumin, M., Niznikiewicz, M., Westin, C. F., Maier, S. E., McCarley, R. W. \& Shenton, M. E. (2006) Fornix integrity and hippocampal volume in male schizophrenic patients. Biological Psychiatry 60:22-31. $[\mathrm{aBC}]$

Kuwako, K., Hosokawa, A., Nishimura, I., Uetsuki, T., Yamada, M., Nada, S., Okada, M. \& Yoshikawa, K. (2005) Disruption of the paternal necdin gene diminishes TrkA signaling for sensory neuron survival. Journal of Neuroscience 25:7090-99. [aBC]

Kuzawa, C. W. (1998) Adipose tissue in human infancy and childhood: An evolutionary perspective. American Journal of Physical Anthropology 27(Suppl.): $177-209 . \quad[\mathrm{aBC}]$

Kwapil, T. R., Barrantes-Vidal, N. \& Silvia, P. J. (in press) The dimensional structure of the Wisconsin Schizotypy Scales: Factor identification and construct validity. Schizophrenia Bulletin. [Advanced access online published September 3, 2007; DOI: 10.1093/schbul/sbm098] [GV]

Kwasnicka-Crawford, D. A., Roberts, W. \& Scherer, S. W. (2007) Characterization of an autism-associated segmental maternal heterodisomy of the chromosome 15q11-13 region. Journal of Autism and Developmental Disorders 37:694-702. [rBC]

Kwon, C. H., Luikart, B. W., Powell, C. M., Zhou, J., Matheny, S. A., Zhang, W., Li, Y., Baker, S. J. \& Parada, L. F. (2006) Pten regulates neuronal arborization and social interaction in mice. Neuron 50:377-88. [aBC]

Lainhart, J. E. (2006) Advances in autism neuroimaging research for the clinician and geneticist. American Journal of Medical Genetics: Part C, Seminars in Medical Genetics 142:33-39. [aBC]

Lainhart, J. E., Bigler, E. D., Bocian, M., Coon, H., Dinh, E., Dawson, G., Deutsch, C. K., Dunn, M., Estes, A., Tager-Flusberg, H., Folstein, S., Hepburn, S., Hyman, S., McMahon, W., Minshew, N., Munson, J., Osann, K., Ozonoff, S., Rodier, P., Rogers, S., Sigman, M., Spence, M. A., Stodgell, C. J. \& Volkmar, F. (2006) Head circumference and height in autism: A study by the collaborative program of excellence in autism. American Journal of Medical Genetics: Part A 140:2257-74. [aBC]

Lajiness-O’Neill, R., Beaulieu, I., Asamoah, A., Titus, J. B., Bawle, E., Ahmad, S., Kirk, J. W. \& Pollack, R. (2006) The neuropsychological phenotype of velocardiofacial syndrome (VCFS): Relationship to psychopathology. Archives of Clinical Neuropsychology 21:175-84. [arBC]

Lalande, M. \& Calciano, M. A. (2007) Molecular epigenetics of Angelman syndrome. Cellular and Molecular Life Sciences 64:947-60. [BJAD]

Landers, M., Calciano, M. A., Colosi, D., Glatt-Deeley, H., Wagstaff, J. \& Lalande, M. (2005) Maternal disruption of Ube3a leads to increased expression of Ube3a-ATS in trans. Nucleic Acids Research 33:3976-84 $[\mathrm{rBC}]$

Landry, R. \& Bryson, S. E. (2004) Impaired disengagement of attention in young children with autism. Journal of Child Psychology and Psychiatry, and Allied Disciplines 45:1115-22. [aBC]

Lane, A., Byrne, M., Mulvany, F., Kinsella, A., Waddington, J. L., Walsh, D., Larkin, C. \& O'Callaghan, E. (1995) Reproductive behaviour in schizophrenia relative to other mental disorders: Evidence for increased fertility in men despite decreased marital rate. Acta Psychiatrica Scandinavica 91:222-28. $[\mathrm{aBC}]$

Langdon, R. (2003) Theory of mind and social dysfunction: Psychotic solipsism versus autistic asociality. In: Individual differences in theory of mind: Implications for typical and atypical development, ed. B. Repacholi \& V. Slaughter, pp. 241-70. Macquarie Monographs in Cognitive Science. Psychology Press. [RL]
(2005) Theory of mind in schizophrenia. In: Other minds: How humans bridge the divide between self and others, ed. B. F. Malle \& S. D. Hodges, pp. 333-42. Guilford Press. [rBC, RL]

Langdon, R. \& Coltheart, M. (1999) Mentalising, schizotypy, and schizophrenia. Cognition 71:43-71. [aBC, KNT]

(2001) Visual perspective-taking and schizotypy: Evidence for a simulation-based account of mentalizing in normal adults. Cognition 82:1-26. [arBC, RL]

(2004) Recognition of metaphor and irony in young adults: The impact of schizotypal personality traits. Psychiatry Research 125:9-20. [aBC]

Langdon, R., Coltheart, M. \& Ward, P. B. (2006a) Empathetic perspective-taking is impaired in schizophrenia: Evidence from a study of emotion attribution and theory of mind. Cognitive Neuropsychiatry 11:133-55. [aBC]

Langdon, R., Coltheart, M., Ward, P. B. \& Catts, S. (2001) Visual and cognitive perspective-taking impairments in schizophrenia: A failure of allocentric simulation? Cognitive Neuropsychiatry 6:241-69. [RL]

(2002) Disturbed communication in schizophrenia: The role of poor pragmatics and poor mind-reading. Psychological Medicine 32:1273-84. [aBC, AT]

Langdon, R., Corner, T., McLaren, J., Coltheart, M. \& Ward, P. B. (2006b) Attentional orienting triggered by gaze in schizophrenia. Neuropsychologia 44:417-29. [arBC, RL]

Lara, D. R., Pinto, O., Akiskal, K. \& Akiskal, H. S. (2006) Toward an integrative model of the spectrum of mood, behavioral and personality disorders based on fear and anger traits: I. Clinical implications. Journal of Affective Disorders $94(1-3): 67-87 . \quad[\mathrm{GV}]$

Larsson, H. J., Eaton, W. W., Madsen, K. M., Vestergaard, M., Olesen, A. V., Agerbo, E., Schendel, D., Thorsen, P. \& Mortensen, P. B. (2005) Risk factors for autism: Perinatal factors, parental psychiatric history, and socioeconomic status. American Journal of Epidemiology 161:916. [aBC]

LaRusso, L. (1978) Sensitivity of paranoid patients to nonverbal clues. Journal of Abnormal Psychology 87:463-71. [arBC]

LaSalle, J. M., Hogart, A. \& Thatcher, K. N. (2005) Rett syndrome: A Rosetta stone for understanding the molecular pathogenesis of autism. International Review of Neurobiology 71:131-65. [aBC]

Laursen, T. M., Munk-Olsen, T., Nordentoft, M. \& Bo Mortensen, P. (2007) A comparison of selected risk factors for unipolar depressive disorder, bipolar affective disorder, schizoaffective disorder, and schizophrenia from a Danish population-based cohort. Journal of Clinical Psychiatry 68:1673-81. [rBC]

Lauterbach, E. C., Spears, T. E., Prewett, M. J., Price, S. T., Jackson, J. G. \& Kirsh, A. D. (1994) Neuropsychiatric disorders, myoclonus, and dystonia in calcification of basal ganglia pathways. Biological Psychiatry 35:345-51. [rBC]

Lawrence, K., Campbell, R., Swettenham, J., Terstegge, J., Akers, R., Coleman, M. \& Skuse, D. (2003) Interpreting gaze in Turner syndrome: Impaired sensitivity to intention and emotion, but preservation of social cueing. Neuropsychologia 41:894-905. [rBC]

Lawrence, P. A. (2006) Men, women, and ghosts in science. PLoS Biology 4:e19. $[\mathrm{aBC}]$

Lawrie, S. M., Whalley, H. C., Job, D. E. \& Johnstone, E. C. (2003) Structural and functional abnormalities of the amygdala in schizophrenia. Annals of the New York Academy of Sciences 985:445-60. [aBC]

Lawson, J., Baron-Cohen, S. \& Wheelwright, S. (2004) Empathising and systemising in adults with and without Asperger Syndrome. Journal of Autism and Developmental Disorders 34:301-10. [aBC]

Lawson, W. (1998) Life behind glass: A personal account of autism spectrum disorder. Jessica Kingsley. $[\mathrm{aBC}]$

Leask, S. J. \& Crow, T. J. (2005) Lateralization of verbal ability in pre-psychotic children. Psychiatry Research 136:35-42. [aBC]

Lee, J. A. \& Lupski, J. R. (2006) Genomic rearrangements and gene copy-number alterations as a cause of nervous system disorders. Neuron 52(1):103-21. [THJB]

Lee, K. H., Farrow, T. F., Spence, S. A. \& Woodruff, P. W. (2004) Social cognition, brain networks and schizophrenia. Psychological Medicine 34:391-400. [aBC]

Lee, M. P. (2003) Genome-wide analysis of epigenetics in cancer. Annals of the New York Academy of Sciences 983:101-109. [aBC]

Lee, S., Walker, C. L., Karten, B., Kuny, S. L., Tennese, A. A., O’Neill, M. A. \& Wevrick, R. (2005) Essential role for the Prader-Willi syndrome protein necdin in axonal outgrowth. Human Molecular Genetics 14:627-37. $[\mathrm{aBC}]$

Leekam, S. R. \& Perner, J. (1991) Does the autistic child have a meta-representational deficit? Cognition 40:203-18. [rBC]

Legg, L. \& Gilbert, P. (2006) A pilot study of gender of voice and gender of voice hearer in psychotic voice hearers. Psychology and Psychotherapy 79: 517-27. [aBC]

Leitman, D. I., Hoptman, M. J., Foxe, J. J., Saccente, E., Wylie, G. R., Nierenberg, J., Jalbrzikowski, M., Lim, K. O. \& Javitt, D. C. (2007) The neural substrates of impaired prosodic detection in schizophrenia and its sensorial antecedents. American Journal of Psychiatry 164:474-82. [MKB] 
Lencz, T., Lambert, C., DeRosse, P., Burdick, K. E., Morgan, T. V., Kane, J. M., Kucherlapati, R. \& Malhotra, A. K. (2007) Runs of homozygosity reveal highly penetrant recessive loci in schizophrenia. Proceedings of the National Academy of Sciences USA 104:19942-47. [MCK]

Leonhard, D. \& Brugger, P. (1998) Creative, paranormal, and delusional thought: A consequence of right hemisphere semantic activation? Neuropsychiatry, Neuropsychology and Behavioral Neurology 11:177-83. [aBC]

Lesch, K. P., Bengel, D., Heils, A., Sabol, S. Z., Greenberg, B. D, Petri, S., Benjamin, J., Müller, C. R., Hamer, D. H. \& Murphy, D. L. (1996) Association of anxiety-related traits with a polymorphism in the serotonin transporter gene regulatory region. Science 274(5292):1527-31. [ST]

Leslie, A. M. \& Thaiss, L. (1992) Domain specificity in conceptual development: Neuropsychological evidence from autism. Cognition 43:225-51. [rBC]

Leung, A. \& Chue, P. (2000) Sex differences in schizophrenia, a review of the literature. Acta Psychiatrica Scandinavica. Supplementum 401:3-38. [aBC]

Levav, I., Lipshitz, I., Novikov, I., Pugachova, I., Kohn, R., Barchana, M., Ponizovsky, A. \& Werner, H. (2007) Cancer risk among parents and siblings of patients with schizophrenia. British Journal of Psychiatry 190 156-61. [aBC]

Lewis, C. M., Levinson, D. F., Wise, L. H., DeLisi, L. E., Straub, R. E., Hovatta, I., Williams, N. M., Schwab, S. G., Pulver, A. E., Faraone, S. V., Brzustowicz, L. M., Kaufmann, C. A., Garver, D. L., Gurling, H. M. D., Lindholm, E., Coon, H., Moises, H. W., Byerley, W., Shaw, S. H., Mesen, A., Sherrington, R., O’Neill, F. A., Walsh, D., Kendler, K. S., Ekelund, J., Paunio, T., Lönnqvist, J., Peltonen, L., O’Donovan, M. C., Owen, M. J., Wildenauer, D. B., Maier, W., Nestadt, G., Blouin, J., Antonarakis, S. E., Mowry, B. J., Silverman, J. M., Crowe, R. R., Cloninger, C. R., Tsuang, M. T., Malaspina, D., HarkavyFriedman, J. M., Svrakic, D. M., Bassett, A. S., Holcomb, J., Kalsi, G., McQuillin, A., Brynjolfson, J., Sigmundsson, T., Petursson, H., Jazin, E. Zoëga, T. \& Helgason, T. (2003) Genome scan meta-analysis of schizophrenia and bipolar disorder, Part II: Schizophrenia. American Journal of Human Genetics 73:34-48. [aBC, MCK]

Lewis, J. D. \& Elman, J. L. (2008) Growth-related neural reorganization and the autism phenotype: A test of the hypothesis that altered brain growth leads to altered connectivity. Developmental Science 11:135-55. [MKB]

Leyfer, O. T., Folstein, S. E., Bacalman, S., Davis, N. O., Dinh, E., Morgan, J., Tager-Flusberg, H. \& Lainhart, J. E. (2006) Comorbid psychiatric disorders in children with autism: Interview development and rates of disorders. Journal of Autism and Developmental Disorders 36:849-61. $[\mathrm{aBC}]$

Leyfer, O. T., Woodruff-Borden, J., Klein-Tasman, B. P., Fricke, J. S. \& Mervis, C. B. (2006) Prevalence of psychiatric disorders in 4 to 16 -year-olds with Williams syndrome. American Journal of Medical Genetics. Part B, Neuropsychiatric Genetics 141:615-22. [rBC]

Libbey, J. E., Sweeten, T. L., McMahon, W. M. \& Fujinami, R. S. (2005) Autistic disorder and viral infections. Journal of Neurovirology 11(1):1-10. [THJB]

Lincoln, A. J., Searcy, Y. M., Jones, W. \& Lord, C. (2007) Social interaction behaviors discriminate young children with autism and Williams syndrome. Journal of the American Academy of Child and Adolescent Psychiatry 46:323-31. [rBC]

Lindenfors, P., Nunn, C. L. \& Barton, R. A. (2007) Primate brain architecture and selection in relation to sex. BMC Biology 5:20. [GV]

Lindsay, R. S., Kobes, S., Knowler, W. C. \& Hanson, R. L. (2002) Genome-wide linkage analysis assessing parent-of-origin effects in the inheritance of birth weight. Human Genetics 110:503-509. [aBC]

Ling, J. Q., Li, T., Hu, J. F., Vu, T. H., Chen, H. L., Qiu, X. W., Cherry, A. M. \& Hoffman, A. R. (2006) CTCF mediates interchromosomal colocalization between Igf2/H19 and Wsbl/Nf1. Science 312:269-72. [rBC]

Linney, Y. M., Murray, R. M., Peters, E. R., MacDonald, A. M., Rijsdijk, F. \& Sham, P. C. (2003) A quantitative genetic analysis of schizotypal personality traits. Psychological Medicine 33:803-16. [aBC]

Lippiello, P. M. (2006) Nicotinic cholinergic antagonists: A novel approach for the treatment of autism. Medical Hypotheses 66:985-90. [aBC]

Lipska, B. K., Swerdlow, N. R., Geyer, M. A., Jaskiw, G. E., Braff, D. L. \& Weinberger, D. R. (1995) Neonatal excitotoxic hippocampal damage in rats cause post-pubertal changes in prepulse inhibition of startle and its disruption by apomorphine. Psychopharmacology (Berlin) 122(1):35-43. [ТНJB]

Lombardo, M. V., Barnes, J. L., Wheelwright, S. J. \& Baron-Cohen, S. (2007) Selfreferential cognition and empathy in autism. PLoS ONE 2:e883. [aBC]

Lord, C., Leventhal, B. L. \& Cook, E. H., Jr. (2001) Quantifying the phenotype in autism spectrum disorders. American Journal of Medical Genetics 105(1):36-38. [CKD]

Lorenz, K. (1963/2002) On aggression. Routledge. (Original work published in 1963) [R-PB

Losh, M. \& Capps, L. (2003) Narrative ability in high-functioning children with autism or Asperger's syndrome. Journal of Autism and Developmental Disorders 33:239-51. [aBC]
(2006) Understanding of emotional experience in autism: Insights from the personal accounts of high-functioning children with autism. Developmental Psychology 42:809-18. [aBC]

Lossie, A. C., Whitney, M. M., Amidon, D., Dong, H. J., Chen, P., Theriaque, D., Hutson, A., Nicholls, R. D., Zori, R. T., Williams, C. A. \& Driscoll, D. J. (2001) Distinct phenotypes distinguish the molecular classes of Angelman syndrome. Journal of Medical Genetics 38:834-45. [aBC]

Luders, E., Rex, D. E., Narr, K. L., Woods, R. P., Jancke, L., Thompson, P. M., Mazziotta, J. C. \& Toga, A. W. (2003) Relationships between sulcal asymmetries and corpus callosum size: Gender and handedness effects. Cerebral Cortex 13:1084-93. [aBC]

Luedi, P. P., Dietrich, F. S., Weidman, J. R., Bosko, J. M., Jirtle, R. L. \& Hartemink, A. J. (2007) Computational and experimental identification of novel human imprinted genes. Genome Research 17:1723-30. [rBC, GV]

Luna, B., Minshew, N. J., Garver, K. E., Lazar, N. A., Thulborn, K. R., Eddy, W. F. \& Sweeney, J. A. (2002) Neocortical system abnormalities in autism: An fMRI study of spatial working memory. Neurology 59:834-40. [aBC]

Lupski, J. R. (2007) Genomic rearrangements and sporadic disease. Nature Genetics 39(Suppl. 7):S43-47. [THJB]

Lutchmaya, S., Baron-Cohen, S. \& Raggatt, P. (2002a) Foetal testosterone and eye contact in 12-month-old human infants. Infant Behavior and Development 25:327-35. [aBC]

(2002b) Foetal testosterone and vocabulary size in 18- and 24-month-old infants. Infant Behavior and Development 24:418-24. [aBC]

Lutchmaya, S., Baron-Cohen, S., Raggatt, P., Knickmeyer, R. \& Manning, J. T. (2004) 2nd to 4th digit ratios, fetal testosterone and estradiol. Early Human Development 77:23-28. [aBC]

Lynn, P. M. Y. \& Davies, W. (2007) The 39,XO mouse as a model for the neurobiology of Turner syndrome and sex-biased neuropsychiatric disorders. Behavioural Brain Research 179:173-82. [rBC]

Machado, C. J. \& Bachevalier, J. (2003) Non-human primate models of childhood psychopathology: The promise and the limitations. Journal of Child Psychology and Psychiatry 44:64-87. [R-PB]

Mackie, S., Millar, J. K. \& Porteous, D. J. (2007) Role of DISC1 in neural development and schizophrenia. Current Opinion in Neurobiology 17(1):95-102. [GV]

Maestro, S., Muratori, F., Cavallaro, M. C., Peo, F., Stern, D., Golse, B. \& PalacioEspaca, F. (2002) Attentional skills during the first 6 months of age in autism spectrum disorder. Journal of the American Academy of Child and Adolescent Psychiatry 41:1239-45. [R-PB]

Mahmoud, G. S. \& Grover, L. M. (2006) Growth hormone enhances excitatory synaptic transmission in area CAl of rat hippocampus. Journal of Neurophysiology 95:2962-74. [aBC]

Malaspina, D., Harlap, S., Fennig, S., Heiman, D., Nahon, D., Feldman, D. \& Susser, E. S. (2001) Advancing paternal age and the risk of schizophrenia Archives of General Psychiatry 58(4):361-67. [THJB]

Malhotra, A. K., Goldman, D., Mazzanti, C., Clifton, A., Breier, A. \& Pickard, D. (1998) A functional serotonin transporter (5-HTT) polymorphism is associated with psychosis in neuroleptic-free schizophrenics. Molecular Psychiatry 3:328-32. [ST]

Manning, J. T. (2002) Digit ratio: A pointer to fertility, behavior, and health. Rutgers University Press. [MV]

Manning, J. T., Baron-Cohen, S., Wheelwright, S. \& Sanders, G. (2001) The 2nd to 4th digit ratio and autism. Developmental Medicine and Child Neurology 43:160-64. [aBC, GV, MV]

Manning, J. T., Bundred, P. E. \& Flanagan, B. F. (2002) The ratio of 2nd to 4th digit length: A proxy for transactivation activity of the androgen receptor gene? Medical Hypotheses 59:334-36. [aBC]

Manning, J. T., Bundred, P. E., Newton, D. J. \& Flanagan, B. F. (2003) The second to fourth digit ratio and variation in the androgen receptor gene. Evolution and Human Behavior 24:399-405. [MV]

Manning, J. T., Scutt, D., Wilson, J. \& Lewis-Jones, D. I. (1998) The ratio of 2nd to 4th digit length: A predictor of sperm numbers and concentrations of testosterone, luteinizing hormone and oestrogen. Human Reproduction 13:3000-3004. [MV]

Manning, J. T., Stewart, A., Bundred, P. E. \& Trivers, R. L. (2004) Sex and ethnic differences in 2 nd to 4 th digit ratio of children. Early Human Development 80:161-68. [aBC]

Mao, R., Jalal, S. M., Snow, K., Michels, V. V., Szabo, S. M. \& Babovic-Vuksanovic, D. (2000) Characteristics of two cases with dup(15)(q11.2-q12): One of maternal and one of paternal origin. Genetics in Medicine 2:131-35. $[\mathrm{rBC}]$

Margetts, C. D. E., Astuti, D., Gentle, D. C., Cooper, W. N., Cascon, A., Catchpoole, D., Robledo, M., Neumann, H. P. H., Latif, F. \& Maher, E. R. (2005) Epigenetic analysis of HIC1:CASP8:FLIP,TSP1:DCR1:DCR2: DR4:DR5:KvDMR1:H19 and preferential 11p15.5 maternal-allele loss in von Hippel-Lindau and sporadic phaeochromocytomas. Endocrine-Related Cancer 12:161-72. [aBC] 
Maric, N., Krabbendam, L., Vollebergh, W., de Graaf, R. \& van Os, J. (2003) Sex differences in symptoms of psychosis in a non-selected, general population sample. Schizophrenia Research 63:89-95. [aBC]

Marjoram, D., Job, D. E., Whalley, H. C., Gountouna, V., McIntosh, A. M., Simonotto, E., Cunningham-Owens, D., Johnstone, E. C. \& Lawrie, S. (2006) A visual joke fMRI investigation into theory of mind and enhanced risk of schizophrenia. NeuroImage 31:1850-58. [aBC]

Marlowe, H. A. (1986) Social intelligence: Evidence for multidimensionality and construct independence. Journal of Educational Psychology 78:52-58. $[\mathrm{rBC}]$

Marshall, C. R., Noor, A., Vincent, J. B., Lionel, A. C., Feuk, L., Skaug, J., Shago, M., Moessner, R., Pinto, D., Ren, Y., Thiruvahindrapduram, B., Fiebig, A., Schreiber, S., Friedman, J., Ketelaars, C. E., Vos, Y. J., Ficicioglu, C., Kirkpatrick, S., Nicolson, R., Sloman, L., Summers, A., Gibbons, C. A., Teebi, A., Chitayat, D., Weksberg, R., Thompson, A., Vardy, C., Crosbie, V., Luscombe, S., Baatjes, R., Zwaigenbaum, L., Roberts, W., Fernandez, B., Szatmari, P., \& Scherer S. W. (2008) Structural variation of chromosomes in autism spectrum disorder. American Journal of Human Genetics 82:477-88. [ [rBC]

Marshall, D. (2000) A study estimating the heritability of 2nd to 4th digit ratio in humans. Unpublished master's thesis, University of Liverpool, United Kingdom. [MV $]$

Martel, M. M., Gobrogge, K. L., Breedlove, S. M. \& Nigg, J. T. (in press) Masculinized finger-length ratios of boys, but not girls, are associated with attention-deficit/hyperactivity disorder. Behavioral Neuroscience. [MV]

Martin, C. L. \& Ledbetter, D. H. (2007) Autism and cytogenetic abnormalities: Solving autism one chromosome at a time. Current Psychiatry Reports $9(2): 141-47 . \quad[\mathrm{WD}]$

Martin, N. D. T., Smith, W. R., Cole, T. J. \& Preece, M. A. (2007) New height, weight and head circumference charts for British children with Williams syndrome. Archives of Disease in Childhood 92:598-601. [rBC]

Mata, I., Sham, P. C., Gilvarry, C. M., Jones, P. B., Lewis, S. W. \& Murray, R. M. (2000) Childhood schizotypy and positive symptoms in schizophrenic patients predict schizotypy in relatives. Schizophrenia Research 44:129-36. [aBC]

Mathes, B., Wood, S. J., Proffitt, T. M., Stuart, G. W., Buchanan, J. A., Velakoulis D., Brewer, W. J., McGorry, P. D. \& Pantelis, C. (2005) Early processing deficits in object working memory in first-episode schizophreniform psychosis and established schizophrenia. Psychological Medicine 35:1053-62. [aBC]

Matthysse, S., Levy, D. L., Kinney, D., Deutsch, C., Lajonchere, C., YurgelunTodd, D., Woods, B. \& Holzman, P. S (1992) Gene expression in mental illness: A navigation chart to future progress. Journal of Psychiatric Research 26(4):461-73. [CKD]

Mattick, J. S. \& Makunin, I. V. (2006) Non-coding RNA. Human Molecular Genetics 15 Spec No 1:R17-29. [rBC]

Mazza, M., De Risio, A., Surian, L., Roncone, R. \& Casacchia, M. (2001) Selective impairments of theory of mind in people with schizophrenia. Schizophrenia Research 47:299-308. [aBC]

McAlonan, G. M., Cheung, V., Cheung, C., Suckling, J., Lam, G. Y., Tai, K. S., Yip, L., Murphy, D. G. M. \& Chua, S. E. (2005) Mapping the brain in autism. A voxel-based MRI study of volumetric differences and intercorrelations in autism. Brain 128:268-76. [aBC]

McAlonan, G. M., Daly, E., Kumari, V., Critchley, H. D., van Amelsvoort, T., Suckling, J., Simmons, A., Sigmundsson, T., Greenwood, K., Russell, A., Schmitz, N., Happé, F., Howlin, P. \& Murphy, D. G. M. (2002) Brain anatomy and sensorimotor gating in Asperger's syndrome. Brain 125:1594-606. $[\mathrm{MKB}, \operatorname{arBC}, \mathrm{MF}]$

McCabe, R., Leudar, I. \& Antaki, C. (2004) Do people with schizophrenia display theory of mind deficits in clinical interactions? Psychological Medicine 34:401-12. [aBC, AT]

McCaffery, P. \& Deutsch, C. K. (2005) Macrocephaly and the control of brain growth in autistic disorders. Progress in Neurobiology 77:38-56. [aBC, CKD]

McCall, T., Chin, S. S., Salzman, K. L. \& Fults, D. W. (2006) Tuberous sclerosis: A syndrome of incomplete tumor suppression. Neurosurgical Focus 20:E3. $[\mathrm{aBC}]$

McClellan, J. M., Susser, E. \& King, M. C. (2007) Schizophrenia: A common disease caused by multiple rare alleles. British Journal of Psychiatry 190: 194-99. [CKD, MCK]

McClure, E. B., Monk, C. S., Nelson, E. E., Zarahn, E., Leibenluft, E., Bilder, R. M., Charney, D. S., Ernst, M. \& Pine, D. S. (2004) A developmental examination of gender differences in brain engagement during evaluation of threat. Biological Psychiatry 55:1047-55. [aBC]

McCreery, C. \& Claridge, G. (1996) A study of hallucination in normal subjects. 1. Self-report. Personality and Individual Differences 21:739-47. [aBC]

McDonald, C., Bullmore, E. T., Sham, P. C., Chitnis, X., Wickham, H., Bramon, E. \& Murray, R. M. (2004) Association of genetic risks for schizophrenia and bipolar disorder with specific and generic brain structural endophenotypes. Archives of General Psychiatry 61:974-84. [aBC]
McDonald, C., Bullmore, E. T., Sham, P. C., Chitnis, X., Suckling, J., MacCabe, J., Walshe, M. \& Murray, R. M. (2005) Regional volume deviations of brain structure in schizophrenia and psychotic bipolar disorder: Computational morphometry study. British Journal of Psychiatry 186:369-77. [aBC]

McDonald, C., Marshall, N., Sham, P. C., Bullmore, E. T., Schulze, K., Chapple, B., Bramon, E., Filbey, F., Quraishi, S., Walshe, M. \& Murray, R. M. (2006) Regional brain morphometry in patients with schizophrenia or bipolar disorder and their unaffected relatives. American Journal of Psychiatry 163(3): 478-87. [WD]

McFadden, D., Westhafer, J. G., Pasanen, E. G., Carlson, C. L. \& Tucker, D. M. (2005) Physiological evidence of hypermasculinization in boys with the inattentive type of attention-deficit/hyperactivity disorder (ADHD). Clinical Neuroscience Research 5:233-45. [MV]

McGlashan, T. H., Pedersen, C. B., Hoffman, R. E. \& Mortensen, P. B. (2006) Fertility of schizophrenia and bipolar probands and their first-degree relatives. Schizophrenia Research 81(Suppl.):S17-S18. [aBC, MCK]

McGrath, J., Feron, F., Burne, T. H. J., Mackay-Sim, A. \& Eyles, D. (2003) The neurodevelopmental hypothesis of schizophrenia: A review of recent developments. Annals of Medicine 35(2):86-93. [THJB]

McGrath, J. J., Hearle, J., Jenner, L., Plant, K., Drummond, A. \& Barkla, J. M. (1999) The fertility and fecundity of patients with psychoses. Acta Psychiatrica Scandinavica 99:441-46. [aBC, MCK]

McIntosh, A. M., Job, D. E., Moorhead, W. J., Harrison, L. K., Whalley, H. C., Johnstone, E. C. \& Lawrie, S. M. (2006) Genetic liability to schizophrenia or bipolar disorder and its relationship to brain structure. American Journal of Medical Genetics. Part B, Neuropsychiatric Genetics 141:76-83. [aBC]

McKay, R., Langdon, R. \& Coltheart, M. (2005) Paranoia, persecutory delusions and attributional biases. Psychiatry Research 136:233-45. [aBC]

McMinn, J., Wei, M., Schupf, N., Cusmai, J., Johnson, E. B., Smith, A. C., Weksberg, R., Thaker, H. M. \& Tycko, B. (2006) Unbalanced placental expression of imprinted genes in human intrauterine growth restriction. Placenta 27:540-49. [aBC]

McNamara, P. (2001) Religion and the frontal lobes. In: Religion in mind: Cognitive perspectives on religious belief, ritual, and experience, ed. J. Andresen, pp. 237-56. Cambridge University Press. [SK]

Mednick, S. A., Huttunen, M. O. \& Machón, R. A. (1994) Prenatal influenza infections and adult schizophrenia. Schizophrenia Bulletin 20:263-67. $[\mathrm{rBC}]$

Meechan, D. W., Maynard, T. M., Gopalakrishna, D., Wu, Y. \& LaMantia, A. S (2007) When half is not enough: Gene expression and dosage in the 22q11 deletion syndrome. Gene Expression 13:299-310. [rBC]

Mendrek, A. (2007) Reversal of normal cerebral sexual dimorphism in schizophrenia: Evidence and speculations. Medical Hypotheses 69:896-902. $[\mathrm{aBC}]$

Meyer, U., Feldon, J., Schedlowski, M. \& Yee, B. K. (2006a) Immunological stress at the maternal-foetal interface: A link between neurodevelopment and adult psychopathology. Brain, Behavior, and Immunity 20(4):378-88. [THJB]

Meyer, U., Nyffeler, M., Engler, A., Urwyler, A., Schedlowski, M., Knuesel, I., Yee, B. K. \& Feldon, J. (2006b) The time of prenatal immune challenge determines the specificity of inflammation-mediated brain and behavioral pathology. Journal of Neuroscience 26(18):4752-62. [THJB]

Meyer, U., Yee, B. K. \& Feldon, J. (2007) The neurodevelopmental impact of prenatal infections at different times of pregnancy: The earlier the worse? Neuroscientist 13:241-56. [rBC]

Meyer-Lindenberg, A., Mervis, C. B. \& Berman, K. F. (2006) Neural mechanisms in Williams syndrome: A unique window to genetic influences on cognition and behaviour. Nature Reviews. Neuroscience 7:380-93. [AT]

Meyer-Lindenberg, A. \& Weinberger, D. R. (2006) Intermediate phenotypes and genetic mechanisms of psychiatric disorders. Nature Reviews Neuroscience 7:818-27. [rBC]

Miller, A. S. \& Kanazawa, S. (2007) Why beautiful people have more daughters. Perigee. [SK]

Miller, A. S. \& Stark, R. (2002) Gender and religiousness: Can socialization explanations be saved? American Journal of Sociology 107:1399-1423. [SK]

Miller, G. F. (2000) Sexual selection for indicators of intelligence. In: The nature of intelligence, ed. G. Bock, J. Goode \& K. Webb, pp. 260-75. Wiley. [DN]

(2001) Aesthetic fitness: How sexual selection shaped artistic virtuosity as a fitness indicator and aesthetic preferences as mate choice criteria. Bulletin of Psychology and the Arts 2:20-25. [BJAD]

Miller, J. L., Couch, J., Schmalfuss, I., He, G., Liu, Y. \& Driscoll, D. J. (2007) Morphological brain abnormalities detected by three dimensional magnetic resonance imaging in Prader-Willi syndrome. American Journal of Medical Genetics: Part A 143:476-83. [aBC]

Mills, J. L., Hediger, M. L., Molloy, C. A., Chrousos, G. P., Manning-Courtney, P., Yu, K. F., Brasington, M. \& England, L. J. (2007) Elevated levels of growth-related hormones in autism and autism spectrum disorder. Clinical Endocrinology 67:230-37. [aBC] 
References/Crespi \& Badcock: Psychosis and autism as diametrical disorders of the social brain

Milne, E., White, S., Campbell, R., Swettenham, J., Hansen, P. \& Ramus, F. (2006) Motion and form coherence detection in autistic spectrum disorder: Relationship to motor control and 2:4 digit ratio. Journal of Autism and Developmental Disorders 36:225-37. [aBC, MV]

Minshew, N. J., Goldstein, G. \& Siegel, D. J. (1997) Neuropsychologic functioning in autism: Profile of a complex information processing disorder. Journal of the International Neuropsychological Society 3:303-16. [aBC]

Mitchell, R. L. C. \& Crow, T. J. (2005) Right hemisphere language functions and schizophrenia: The forgotten hemisphere? Brain 128:963-78. [aBC]

Mitchell, T. N., Free, S. L., Merschhemke, M., Lemieux, L., Sisodiya, S. M. \& Shorvon, S. D. (2003) Reliable callosal measurement: Population normative data confirm sex-related differences. American Journal of Neuroradiology $24: 410-18 . \quad[\mathrm{aBC}]$

Miyazaki, K., Narita, N., Sakuta, R., Miyahara, T., Naruse, H., Okado, N. \& Narita, M. (2004) Serum neurotrophin concentrations in autism and mental retardation: A pilot study. Brain and Development 26:292-95. $[\mathrm{aBC}]$

Mlakar, J., Jensterle, J. \& Frith, C. (1994) Central monitoring deficiency and schizophrenic symptoms. Psychological Medicine 24:557-64. [AA-A]

Modahl, C., Green, L. A., Fein, D., Waterhouse, L., Feinstein, C., Morris, M. \& Levin, H. (1998) Plasma oxytocin levels in autistic children. Biological Psychiatry 43(4):270-77. [R-PB, LMG]

Mohr, C., Krummenacher, P., Landis, T., Sandor, P. S., Fathi, M. \& Brugger, P. (2005) Psychometric schizotypy modulates levodopa effects on lateralized lexical decision performance. Journal of Psychiatric Research 39:241-50. [aBC]

Mohr, C., Röhrenbach, C. M., Laska, M. \& Brugger, P. (2001) Unilateral olfactory perception and magical ideation. Schizophrenia Research 47:255-64. [aBC]

Moises, H. W., Zoega, T. \& Gottesman, I. I. (2002) The glial growth factors deficiency and synaptic destabilization hypothesis of schizophrenia. $B M C$ Psychiatry 2:8. [aBC, LMO]

Molesworth, C. J., Bowler, D. M. \& Hampton, J. A. (2005) The prototype effect in recognition memory: Intact in autism? Clinical Child Psychology and Psychiatry 46(6):661-72. [TV]

Money, J. (1993) Specific neuro-cognitive impairments associated with turner $(45, \mathrm{X})$ and Klinefelter $(47, \mathrm{XXY})$ syndromes: A review. Social Biology 40 : 147-51. [rBC]

Monk, D. \& Moore, G. E. (2004) Intrauterine growth restriction - Genetic causes and consequences. Seminars in Fetal and Neonatal Medicine 9:371-78. [aBC]

Montag, C., Heinz, A., Kunz, D. \& Gallinat, J. (2007) Self-reported empathic abilities in schizophrenia. Schizophrenia Research 92:85-89. [aBC, RL]

Moore, C. (2004) Different connections. Sunday Times, October 10, 2004, p. 10. $[\mathrm{rBC}]$

Moore, T. \& Haig, D. (1991) Genomic imprinting in mammalian development: A parental tug-of-war. Trends in Genetics 7:45-49. [BJAD]

Moriarty, P. J., Lieber, D., Bennett, A., White, L., Parrella, M., Harvey, P. D. \& Davis, K. L. (2001) Gender differences in poor outcome patients with lifelong schizophrenia. Schizophrenia Bulletin 27:103-13. [aBC]

Mors, O., Mortensen, P. B. \& Ewald, H. (2001) No evidence of increased risk for schizophrenia or bipolar affective disorder in persons with aneuploidies of the sex chromosomes. Psychological Medicine 31:425-30. [rBC]

Mottron, L., Dawson, M., Soulières, I., Hubert, B., Burack, J. A. (2006) Enhanced perceptual functioning in autism: An update, and eight principles of autistic perception. Journal of Autism and Developmental Disorders 36:27-43. [MKB, aBC]

Mouridsen, S. E. \& Hansen, M. B. (2002) Neuropsychiatric aspects of Sotos syndrome. A review and two case illustrations. European Child and Adolescent Psychiatry 11:43-48. [rBC]

Mouridsen, S. E., Rich, B. \& Isager, T. (2008) Psychiatric disorders in adults diagnosed as children with atypical autism. A case control study. Journal of Neural Transmission 115(1):135-38. [aBC, SvR, GV]

Moy, S. S., Nadler, J. J., Young, N. B., Perez, A., Holloway, L. P., Barbaro, R. P., Wilson, L. M., Threadgill, D. W., Lauder, J. M., Magnuson, T. R. \& Crawley, J. M. (2007) Mouse behavioral tasks relevant to autism: Phenotypes of 10 inbred strains. Behavioral Brain Research 176(1):4-20. [ТНJB]

Mraz, K. D., Green, J., Dumont-Mathieu, T., Makin, S. \& Fein, D. (2007) Correlates of head circumference growth in infants later diagnosed with autism spectrum disorders. Journal of Child Neurology 22:700-13. [arBC]

Mucci, A., Galderisi, S., Bucci, P., Tresca, E., Forte, A., Koenig, T. \& Maj, M. (2005) Hemispheric lateralization patterns and psychotic experiences in healthy subjects. Psychiatry Research 139:141-54. [aBC]

Mueser, K. T., Bellack, A. S., Douglas, M. S. \& Morrison, R. L. (1991) Prevalence and stability of social skill deficits in schizophrenia. Schizophrenia Research $5: 167-76 . \quad[\mathrm{ST}]$

Mukaddes, N. M. \& Herguner, S. (2007) Autistic disorder and 22q11.2 duplication. World Journal of Biological Psychiatry 8:127-30. [rBC]

Murray, D., Lesser, M. \& Lawson, W. (2005) Attention, monotropism and the diagnostic criteria for autism. Autism 9:139-56. [aBC]
Nakamura, M., Matsushima, E., Ohta, K., Ando, K. \& Kojima, T. (2003) Relationship between attention and arousal level in schizophrenia. Psychiatry and Clinical Neurosciences 57:472-77. [aBC]

Narr, K. L., Bilder, R. M., Toga, A. W., Woods, R. P., Rex, D. E., Szeszko, P. R., Robinson, D., Sevy, S., Gunduz-Bruce, H., Wang, Y. P., DeLuca, H. \& Thompson, P. M. (2005) Mapping cortical thickness and gray matter concentration in first episode schizophrenia. Cerebral Cortex 15:708-19. [aBC]

Narr, K. L., Thompson, P. M., Szeszko, P., Robinson, D., Jang, S., Woods, R. P., Kim, S., Hayashi, K. M., Asunction, D., Toga, A. W. \& Bilder, R. M. (2004) Regional specificity of hippocampal volume reductions in first-episode schizophrenia. NeuroImage 21:1563-75. [aBC]

Narr, K. L., van Erp, T. G., Cannon, T. D., Woods, R. P., Thompson, P. M., Jang, S., Blanton, R., Poutanen, V. P., Huttunen, M., Lönnqvist, J., StanderksjoldNordenstam, C. G., Kaprio, J., Mazziotta, J. C. \& Toga, A. W. (2002) A twin study of genetic contributions to hippocampal morphology in schizophrenia. Neurobiology of Disease 11:83-95. [aBC]

Nation, K. \& Penny, S. (2008) Sensitivity to eye gaze in autism: Is it normal? Is it automatic? Is it social? Development and Psychopathology 20:79-97. [rBC]

Nelson, K. B. (2001) Toward a biology of autism: Possible role of certain neuropeptides and neurotrophins. Clinical Neuroscience Research 1:300-306. [aBC]

Nesse, R. M. (2004) Cliff-edged fitness functions and the persistence of schizophrenia. Behavioral and Brain Sciences 27:862-63. [RP-B, aBC] (2005) Maladaptation and natural selection. Quarterly Review of Biology 80: $62-70 . \quad[\mathrm{aBC}]$

Nesse, R. M., Stearns, S. C. \& Omenn, G. S. (2006) Medicine needs evolution. Science 311(5764):1071. [GV]

Nettle, D. (2001) Strong imagination: Madness, creativity and human nature. Oxford University Press. [aBC]

(2006) Schizotypy and mental health amongst poets, visual artists, and mathematicians. Journal of Research in Personality 40:876-90. [arBC, DN] (2007) Empathizing and systemizing: What are they, and what do they contribute to our understanding of psychological sex differences? British Journal of Psychology, Part 2 98:237-55. [aBC]

Nettle, D. \& Clegg, H. (2006) Schizotypy, creativity and mating success in humans. Proceedings of the Royal Society of London Series B, Biological Sciences 273:611-15. [aBC, BJAD, DN, GV]

(2007) Personality, mating strategies and mating intelligence. In: Mating intelligence: Sex, relationships and the mind's reproductive system, ed. G. Geher \& G. F. Miller, pp. 121-34. Erlbaum. [DN]

Niculescu, A. B. (2005) Genomic studies of mood disorders - The brain as a muscle? Genome Biology 6:215. [aBC]

Niebauer, C. L. (2004) Handedness and the fringe of consciousness: Strong handers ruminate while mixed handers self-reflect. Consciousness and Cognition 13:730-45. $\quad[\mathrm{aBC}]$

Niemi, L. T., Suvisaari, J. M., Haukka, J. K. \& Lönnqvist, J. K. (2005) Childhood growth and future development of psychotic disorder among Helsinki high-risk children. Schizophrenia Research 76:105-39. [aBC]

Nilsson, E., Stålberg, G., Lichtenstein, P., Cnattingius, S., Olausson, P. O. \& Hultman, C. M. (2005) Fetal growth restriction and schizophrenia: A Swedish twin study. Twin Research and Human Genetics 8:402-408. [aBC]

Nishimura, K., Nakamura, K., Anitha, A., Yamada, K., Tsujii, M., Iwayama, Y., Hattori, E., Toyota, T., Takei, N., Miyachi, T., Iwata, Y., Suzuki, K., Matsuzaki, H., Kawai, M., Sekine, Y., Tsuchiya, K., Sugihara, G., Suda, S., Ouchi, Y., Sugiyama, T., Yoshikawa, T. \& Mori, N. (2007a) Genetic analyses of the brainderived neurotrophic factor (BDNF) gene in autism. Biochemical and Biophysical Research Communications 356:200-206. [aBC]

Nishimura, Y., Martin, C. L., Vazquez-Lopez, A., Spence, S. J., Alvarez-Retuerto, A. I., Sigman, M., Steindler, C., Pellegrini, S., Schanen, N. C., Warren, S. T. \& Geschwind, D. H. (2007b) Genome-wide expression profiling of lymphoblastoid cell lines distinguishes different forms of autism and reveals shared pathways. Human Molecular Genetics 16:1682-98. [rBC]

Nithianantharajah, J. \& Hannan, A. J. (2007) Dynamic mutations as digital genetic modulators of brain development, function and dysfunction. Bioessays 29(6):525-35. [THJB]

Noesselt, T., Driver, J., Heinze, H. J. \& Dolan, R. (2005) Asymmetrical activation in the human brain during processing of fearful faces. Current Biology 15:424-29. [aBC]

Nomura, T., Kimura, M., Horii, T., Morita, S., Soejima, H., Kudo, S. \& Hatada, I. (2008) MeCP2-dependent repression of an imprinted miR-184 released by depolarization. Human Molecular Genetics 17:1192-99. [rBC]

Nowicki, S. T., Tassone, F., Ono, M. Y., Ferranti, J., Croquette, M. F., GoodlinJones, B. \& Hagerman, R. J. (2007) The Prader-Willi phenotype of Fragile $\mathrm{X}$ syndrome. Journal of Developmental and Behavioral Pediatrics 28:13338. $[\mathrm{rBC}]$

Nurmi, E. L., Amin, T., Olson, L. M., Jacobs, M. M., McCauley, J. L., Lam, A. Y., Organ, E. L., Folstein, S. E., Haines, J. L. \& Sutcliffe, J. S. (2003) Dense 
References/Crespi \& Badcock: Psychosis and autism as diametrical disorders of the social brain

linkage disequilibrium mapping in the 15q11-q13 maternal expression domain yields evidence for association in autism. Molecular Psychiatry 8:624. [rBC]

Nurmi, E. L., Bradford, Y., Chen, Y., Hall, J., Arnone, B., Gardiner, M. B.,

Hutcheson, H. B., Gilbert, J. R., Pericak-Vance, M. A., Copeland-Yates, S. A., Michaelis, R. C., Wassink, T. H., Santangelo, S. L., Sheffield, V. C., Piven, J. Folstein, S. E., Haines, J. L. \& Sutcliffe, J. S. (2001) Linkage disequilibrium at the Angelman syndrome gene UBE3A in autism families. Genomics $77: 105-13 . \quad[\mathrm{aBC}]$

Nydén, A., Carlsson, M., Carlsson, A. \& Gillberg, C. (2004) Interhemispheric transfer in high-functioning children and adolescents with autism spectrum disorders: A controlled pilot study. Developmental Medicine and Child Neurology 46:448-54. [aBC]

Nylander, L. \& Gillberg, C. (2001) Screening for autism spectrum disorders in adult psychiatric out-patients: A preliminary report. Acta Psychiatrica Scandinavica 103:428-34. [aBC]

O'Neill, J. L. (1999) Through the eyes of aliens: A book about autistic people. Jessica Kinglsey. $\quad[\mathrm{aBC}]$

O'Shea, A. G., Fein, D. A., Cillessen, A. H., Klin, A. \& Schultz, R. T. (2005) Source memory in children with autism spectrum disorders. Developmental Neuropsychology 27:337-60. [aBC]

Ohama, K., Ueda, K., Okamoto, E., Takenaka, M. \& Fujiwara, A. (1986) Cytogenetic and clinicopathologic studies of partial moles. Obstetrics and Gynecology 68:259-62. [aBC]

Ohnishi, T., Matsuda, H., Hashimoto, T., Kunihiro, T., Nishikawa, M., Uema, T. \& Sasaki, M. (2000) Abnormal regional cerebral blood flow in childhood autism. Brain 123:1838-44. [aBC]

Osawa, J., Sugie, H., Fukuda, T., Ito, M., Sugie, Y. \& Ohzeki, T. (2005) Evaluation of the 2nd to 4th digit ratio in patients with autism. [Article in Japanese.] No To Hattatsu [Brain and Development] 37:424-25. [MV]

Oudejans, C. B. M., Mulders, J., Lachmeijer, A. M. A., van Dijk, M., Könst, A. A. M., Westerman, B. A., van Wijk, I. J., Leegwater, P. A. J., Kato, H. D., Matsuda, T., Wake, N., Dekker, G. A., Pals, G., ten Kate, L. P. \& Blankenstein, M. A. (2004) The parent-of-origin effect of 10q22 in pre-eclamptic females coincides with two regions clustered for genes with down-regulated expression in androgenetic placentas. Molecular Human Reproduction 10:589-98. [aBC]

Palomino, A., Vallejo-Illarramendi, A., González-Pinto, A., Aldama, A., GonzálezGómez, C., Mosquera, F., González-García, G. \& Matute, C. (2006) Decreased levels of plasma BDNF in first-episode schizophrenia and bipolar disorder patients. Schizophrenia Research 86:321-22. [aBC, LMO]

Paradiso, S., Andreasen, N. C., Crespo-Facorro, B., O’Leary, D. S., Watkins, G. L., Boles Ponto, L. L. \& Hichwa, R. D. (2003) Emotions in unmedicated patients with schizophrenia during evaluation with positron emission tomography. American Journal of Psychiatry 160:1775-83. [aBC]

Park, S., Lenzenweger, M. F., Püschel, J. \& Holzman, P. S. (1996) Attentional inhibition in schizophrenia and schizotypy, a spatial negative priming study. Cognitive Neuropsychiatry 1:125-49. [aBC]

Paul, S. N., Kato, B. S., Cherkas, L. F., Andrew, T. \& Spector, T. D. (2006) Heritability of the second to fourth digit ratio (2d:4d): A twin study. Twin Research and Human Genetics 9:215-19. [MV]

Pearson, C. E., Nichol Edamura, K. \& Cleary, J. D. (2005) Repeat instability: Mechanisms of dynamic mutations. Nature Reviews. Genetics 6(10):729-42 [THJB]

Penn, H. E. (2006) Neurobiological correlates of autism: A review of recent research. Child Neuropsychology 12:57-79. [aBC]

Pennington, B. F. \& Ozonoff, S. (1996) Executive functions and developmental psychopathology. Journal of Child Psychology and Psychiatry 37:51-87. [R-PB]

Perrin, M. C., Brown, A. S. \& Malaspina, D. (2007) Aberrant epigenetic regulation could explain the relationship of paternal age to schizophrenia. Schizophrenia Bulletin 33(6):1270-73. [THJB, rBC]

Perry, G. H., Tchinda, J., McGrath, S. D., Zhang, J., Picker, S. R., Cáceres, A. M., Iafrate, A. J., Tyler-Smith, C., Scherer, S. W., Eichler, E. E., Stone, A. C. \& Lee, C. (2006) Hotspots for copy number variation in chimpanzees and humans. Proceedings of the National Academy of Sciences USA 103(21): 8006-11. [CKD]

Perry, W., Minassian, A., Lopez, B., Maron, L. \& Lincoln A. (2007) Sensorimotor gating deficits in adults with autism. Biological Psychiatry 61:482-86. [MKB]

Peters, M., Mackenzie, K. \& Bryden, P. (2002) Finger length and distal finger extent patterns in humans. American Journal of Physical Anthropology 117: 209-17. [MV]

Peters, S. U., Beaudet, A. L., Madduri, N. \& Bacino, C. A. (2004) Autism in Angelman syndrome: Implications for autism research. Clinical Genetics 66(6):530-36. [aBC, CKD]

Petty, L. K., Ornitz, E. M., Michelman, J. G. \& Zimmerman, E. G. (1984) Autistic children who become schizophrenic. Archives of General Psychiatry 41: $129-35 . \quad[\mathrm{ST}]$
Philippi, A., Roschmann, E., Tores, F., Lindenbaum, P., Benajou, A., GermainLeclerc, L., Marcaillou, C., Fontaine, K., Vanpeene, M., Roy, S., Maillard, S., Decaulne, V., Saraiva, J. P., Brooks, P., Rousseau, F. \& Hager, J. (2005) Haplotypes in the gene encoding protein kinase c-beta (PRKCBl) on chromosome 16 are associated with autism. Molecular Psychiatry 10:950-60. [aBC]

Phillips, M. L., Senior, C. \& David, A. S. (2000) Perception of threat in schizophrenics with persecutory delusions: An investigation using visual scan paths. Psychological Medicine 30:157-67. [aBC]

Phillips, M. L., Williams, L., Senior, C., Bullmore, E. T., Brammer, M. J., Andrew, C., Williams, S. C. \& David, A. S. (1999) A differential neural response to threatening and non-threatening negative facial expressions in paranoid and non-paranoid schizophrenics. Psychiatry Research 92:11-31. [aBC]

Piccinelli, M. \& Wilkinson, G. (2000) Gender differences in depression. Critical review. British Journal of Psychiatry 177:486-92. [aBC]

Pickup, G. J. (2006) Theory of mind and its relation to schizotypy. Cognitive Neuropsychiatry 11(2):177-92. [arBC, SvR]

Pickup, G. J. \& Frith, C. D. (2001) Theory of mind impairments in schizophrenia: Symptomatology, severity and specificity. Psychological Medicine 31: 207-20. [aBC]

Piefke, M. \& Fink, G. R. (2005) Recollections of one's own past: The effects of aging and gender on the neural mechanisms of episodic autobiographical memory. Anatomy and Embryology 210:497-512. [aBC]

Pieper, A. A., Wu, X., Han, T. W., Estill, S. J., Dang, Q., Wu, L. C., Reece-Fincanon, S., Dudley, C. A., Richardson, J. A., Brat, D. J. \& McKnight, S. L. (2005) The neuronal PAS domain protein 3 transcription factor controls FGFmediated adult hippocampal neurogenesis in mice. Proceedings of the National Academy of Sciences USA 102:14052-57. [aBC]

Pies, R. (2007) The historical roots of the bipolar spectrum: Did Aristotle anticipate Kraepelin's broad concept of manic-depression? Journal of Affective Disorders 100:7-11. [MKB]

Pilcher, D. L., Hammock, E. A. \& Hopkins, W. D. (2001) Cerebral volumetric asymmetries in non-human primates: A magnetic resonance imaging study. Laterality 6:165-79. [aBC]

Pilowsky, T., Yirmiya, N., Arbelle, S. \& Mozes, T. (2000) Theory of mind abilities of children with schizophrenia, children with autism, and normally developing children. Schizophrenia Research 42:145-55. [aBC]

Pinkham, A. E., Hopfinger, J. B., Pelphrey, K. A., Piven, J. \& Penn, D. L. (2008) Neural bases for impaired social cognition in schizophrenia and autism spectrum disorders. Schizophrenia Research 99(1-3):164-75. [SvR]

Pinkham, A. E., Penn, D. L., Perkins, D. O. \& Lieberman, J. (2003) Implications for the neural basis of social cognition for the study of schizophrenia. American Journal of Psychiatry 160:815-24. [aBC]

Piven, J., Bailey, J., Ranson, B. J. \& Arndt, S. (1997) An MRI study of the corpus callosum in autism. American Journal of Psychiatry 154:1051-56. [aBC]

Pizzagalli, D., Lehmann, D. \& Brugger, P. (2001) Lateralized direct and indirect semantic priming effects in subjects with paranormal experiences and beliefs. Psychopathology 34:75-80. [aBC]

Pizzagalli, D., Lehmann, D., Gianotti, L., Koenig, T., Tanaka, H., Wackermann, J. \& Brugger, P. (2000) Brain electric correlates of strong belief in paranormal phenomena: Intracerebral EEG source and regional Omega complexity analyses. Psychiatry Research 100:139-54. [aBC]

Plagge, A., Gordon, E., Dean, W., Boiani, R., Cinti, S., Peters, J. \& Kelsey, G. (2004) The imprinted signaling protein $\mathrm{XL}$ alpha s is required for postnatal adaptation to feeding. Nature Genetics 36:818-26. [aBC]

Planansky, K. \& Johnston, R. (1962) The incidence and relationship of homosexual and paranoid features in schizophrenia. Journal of Mental Science 108:604-15. [aBC]

Plante, I., Davidovic, L., Ouellet, D. L., Gobeil, L., Tremblay, S., Khandjian, E. W. \& Provost, P. (2006) Dicer-derived MicroRNAs are utilized by the Fragile X mental retardation protein for assembly on target RNAs. Journal of Biomedicine and Biotechnology 2006:64347. [rBC]

Potash, J. B. (2006) Carving chaos: Genetics and the classification of mood and psychotic syndromes. Harvard Review of Psychiatry 14:47-63. [aBC]

Potocki, L., Bi, W., Treadwell-Deering, D., Carvalho, C. M. B., Eifert, A., Friedman, E. M., Glaze, D., Krull, K., Lee, J. A., Lewis, R. A., MendozaLondono, R., Robbins-Furman, P., Shaw, C., Shi, X., Weissenberger, G., Withers, M., Yatsenko, S. A., Zackai, E. H., Stankiewicz, P. \& Lupski, J. R. (2007) Characterization of Potocki-Lupski syndrome (dup(17)(p11.2p11.2)) and delineation of a dosage-sensitive critical interval that can convey an autism phenotype. American Journal of Human Genetics 80:633-49. [rBC]

Povinelli, D. J. (2000) Folk physics for apes: The chimpanzee's theory of how the world works. Oxford University Press. [rBC]

Preti, A., Sardu, C. \& Piga, A. (2007) Mixed-handedness is associated with the reporting of psychotic-like beliefs in a non-clinical Italian sample. Schizophrenia Research 92:15-23. [aBC]

Previc, F. H. (2007) Prenatal influences on brain dopamine and their relevance to the rising incidence of autism. Medical Hypotheses 68:46-60. [aBC] 
Pring, L. (2005) Savant talent. Developmental Medicine and Child Neurology 47:500-503. [aBC]

Procopio, M., Davies, R. J. \& Marriott, P. (2006) The hormonal environment in utero as a potential aetiological agent for schizophrenia. European Archives of Psychiatry and Clinical Neuroscience 256(2):77-81. [GV, MV]

Prokosch, M., Yeo, R. \& Miller, G. F. (2005) Intelligence tests with higher gloadings show higher correlations with body symmetry: Evidence for a general fitness factor mediated by developmental stability. Intelligence 33:203-13. [DN]

Putz, D. A., Gaulin, S. J. C., Sporter, R. J. \& McBurney, D. H. (2004) Sex hormones and finger length: What does 2D:4D indicate? Evolution and Human Behavior 25:182-99. [MV]

Pylyshyn, Z. (1985) Computation and cognition. MIT Press. [WF]

Quintana, J., Davidson, T., Kovalik, E., Marder, S. R. \& Mazziotta, J. C. (2001) A compensatory mirror cortical mechanism for facial affect processing in schizophrenia. Neuropsychopharmacology 25:915-24. [aBC]

Ramesh, A. \& Murty, J. S. (1977) Variation and inheritance of relative length of index finger in man. Annals of Human Biology 4:479-84. [MV]

Rampersaud, E., Morris, R. W., Weinberg, C. R., Speer, M. C. \& Martin, E. R. (2007) Power calculations for likelihood ratio tests for offspring genotype risks, maternal effects, and parent-of-origin (POO) effects in the presence of missing parental genotypes when unaffected siblings are available. Genetic Epidemiology 31:18-30. [rBC]

Randerson, J. (2002) All in the mind? New Scientist 176:10. [rBC]

Rapoport, J. L., Addington, A. M., Frangou, S. \& Psych, M. R. (2005) The neurodevelopmental model of schizophrenia: Update 2005. Molecular Psychiatry 10:434-49. [aBC]

Räsänen, S., Pakaslahti, A., Syvälahti, R., Jones, P. B. \& Isohanni, M. (2000) Sex differences in schizophrenia: A review. Nordic Journal of Psychiatry 54:37-45. [aBC]

Ratcliffe, S. G., Butler, G. E. \& Jones, M. (1990) Edinburgh study of growth and development of children with sex chromosome abnormalities. IV. Birth Defects Original Article Series 26:1-44. [rBC]

Redcay, E. \& Courchesne, E. (2005) When is the brain enlarged in autism? A metaanalysis of all brain size reports. Biological Psychiatry 58:1-9. [aBC]

Rees, S. \& Inder, T. (2005) Fetal and neonatal origins of altered brain development Early Human Development 81:753-61. [aBC]

Reichenberg, A., Gross, R., Weiser, M., Bresnahan, M., Silverman, J., Harlap, S., Rabinowitz, J., Shulman, C., Malaspina, D., Lubin, G., Knobler, H. Y., Davidson, M. \& Susser, E. (2006) Advancing paternal age and autism. Archives of General Psychiatry 63(9):1026-32. [THJB, rBC, GV]

Reik, W., Constancia, M., Fowden, A., Anderson, N., Dean, W., Ferguson-Smith, A., Tycko, B. \& Sibley, C. (2003) Regulation of supply and demand for maternal nutrients in mammals by imprinted genes. Journal of Physiology 547:35-44. [aBC]

Reik, W. \& Walter, J. (2001) Genomic imprinting: Parental influence on the genome. Nature Reviews. Genetics 2(1):21-32. [GV]

Rennison, N. (2005) Sherlock Holmes: The unauthorized biography. Atlantic. [rBC]

Richardson, A. J. (1994) Dyslexia, handedness and syndromes of psychosis-proneness. International Journal of Psychophysiology 18:251-63. [aBC]

Richell, R. A., Mitchell, D. G., Newman, C., Leonard, A., Baron-Cohen, S. \& Blair, R. J. (2003) Theory of mind and psychopathy: Can psychopathic individuals read the "language of the eyes"? Neuropsychologia 41:523-26. [AT]

Rickard, I. J., Russell, A. F. \& Lummaa, V. (2007) Producing sons reduces lifetime reproductive success of subsequent offspring in pre-industrial Finns. Proceedings of the Royal Society of London, Series B: Biological Sciences 274:2981-88. [arBC]

Riedel, H. (2004) GRB10 exceeding the boundaries of a common signaling adapter. Frontiers in Bioscience 9:603-18. [aBC]

Riikonen, R., Makkonen, I., Vanhala, R., Turpeinen, U., Kuikka, J. \& Kokki, H. (2006) Cerebrospinal fluid insulin-like growth factors IGF-1 and IGF-2 in infantile autism. Developmental Medicine and Child Neurology 48(9):751-55. [GV]

Rilling, J. K. \& Insel, T. R. (1999) Differential expansion of neural projection systems in primate brain evolution. Neuroreport 10:1453-59. [aBC]

Rim, Y. (1994) Impulsivity, venturesomeness, empathy and schizotypy. Personality and Individual Differences 17:853-54. [aBC]

Rimmer, J. \& Jacobsen, B. (1976) Differential fertility of adopted schizophrenics and their half-siblings. Acta Psychiatrica Scandinavica 54:161-66. [aBC, MCK]

Rinaldi, T., Silberberg, G. \& Markram, H. (2008) Hyperconnectivity of local neocortical microcircuitry induced by prenatal exposure to valproic acid. Cerebral Cortex 18:763-70. [aBC]

Rinehart, N. J., Bradshaw, J. L., Brereton, A. V. \& Tonge, B. J. (2002a) A clinical and neurobehavioural review of high-functioning autism and Asperger's disorder. Australian and New Zealand Journal of Psychiatry 36:762-70. [aBC]

(2002b) Lateralization in individuals with high-functioning autism and Asperger's disorder: A frontostriatal model. Journal of Autism and Developmental Disorders 32:321-31. [aBC]
Rio, M., Clech, L., Amiel, J., Faivre, L., Lyonnet, S., Le Merrer, M., Odent, S., Lacombe, D., Edery, P., Brauner, R., Raoul, O., Gosset, P., Prieur, M., Vekemans, M., Munnich, A., Colleaux, L. \& Cormier-Daire, V. (2003) Spectrum of NSD1 mutations in Sotos and Weaver syndromes. Journal of Medical Genetics 40:436-40. [rBC]

Ripoll, N., Bronnec, M. \& Bourin, M. (2004) Nicotinic receptors and schizophrenia. Current Medical Research and Opinion 20:1057-74. [aBC]

Rippon, G., Brock, J., Brown, C. \& Boucher, J. (2007) Disordered connectivity in the autistic brain: Challenges for the "new psychophysiology." International Journal of Psychophysiology 63(2):164-72. [CKD]

Ristic, J., Mottron, L., Friesen, C. K., Iarocci, G., Burack, J. A. \& Kingstone, A. (2005) Eyes are special but not for everyone: The case of autism. Cognitive Brain Research 24:715-18. [aBC, RL]

Rodríguez-Criado, G., Magano, L., Segovia, M., Gurrieri, F., Neri, G., GonzálezMeneses, A., Gómez de Terreros, I., Valdéz, R., Gracia, R. \& Lapunzina, P. (2005) Clinical and molecular studies on two further families with SimpsonGolabi-Behmel syndrome. American Journal of Medical Genetics: Part A 138:272-77. [rBC]

Rogers, S. J., Hepburn, S. \& Wehner, E. (2003) Parent reports of sensory symptoms in toddlers with autism and those with other developmental disorders. Journal of Autism and Developmental Disorders 33:631-42. $[\mathrm{aBC}]$

Rojas, D. C., Smith, J. A., Benkers, T. L., Camou, S. L., Reite, M. L. \& Rogers, S. J. (2004) Hippocampus and amygdala volumes in parents of children with autistic disorder. American Journal of Psychiatry 161:2038-44. [MKB]

Ronald, A., Happé, F., Bolton, P., Butcher, L. M., Price, T. S., Wheelwright, S., Baron-Cohen, S. \& Plomin, R. (2006) Genetic heterogeneity between the three components of the autism spectrum: A twin study. Journal of the American Academy of Child and Adolescent Psychiatry 45(6):691-99. [aBC, BJAD, LMG]

Roof, E., Stone, W., MacLean, W., Feurer, I. D., Thompson, T. \& Butler, M. G. (2000) Intellectual characteristics of Prader-Willi syndrome: Comparison of genetic subtypes. Journal of Intellectual Disability Research 44:25-30. [arBC]

Ropers, H. H. (2007) New perspectives for the elucidation of genetic disorders. American Journal of Human Genetics 81(2):199-207. [CKD]

Rosenfeld, H. A. (1965) Psychotic states: A psychoanalytical approach. Maresfield Reprints. [R-PB]

Ross, C. A., Margolis, R. L., Reading, S. A. J., Pletnikov, M. \& Coyle, J. T. (2006a) Neurobiology of schizophrenia. Neuron 52:139-53. [aBC]

Ross, R. G., Heinlein, S. \& Tregellas, H. (2006b) High rates of comorbidity are found in childhood-onset schizophrenia. Schizophrenia Research 88:90-95. $[\mathrm{aBC}]$

Rourke, B. P., Bakker, D. J., Fisk, J. L. \& Strang, J. D. (1983) Child neuropsychology. Guilford Press. [SvR]

Rowe, D. A., Bullock, P. R., Polkey, C. E. \& Morris, R. G. (2001) "Theory of mind" impairments and their relationship to executive functioning following frontal lobe lesions. Brain 124:600-16. [R-PB]

Royo, H., Bortolin, M., Seitz, H. \& Cavaillé, J. (2006) Small non-coding RNAs and genomic imprinting. Cytogenetic and Genome Research 113:99-108. $[\mathrm{rBC}]$

Rubenstein, J. L. \& Merzenich, M. M. (2003) Model of autism: Increased ratio of excitation/inhibition in key neural systems. Genes, Brain, and Behavior 2:255-67. [aBC, CKD]

Russell, T. A., Reynaud, E., Herba, C., Morris, R. \& Corcoran, R. (2006) Do you see what I see? Interpretations of intentional movement in schizophrenia. Schizophrenia Research 81:101-11. [aBC, RL]

Russell, T. A., Reynaud, E., Kucharska-Pietura, K., Ecker, C., Benson, P. J., Zelaya, F., Giampietro, V., Brammer, M., David, A. \& Phillips, M. L. (2007) Neural responses to dynamic expressions of fear in schizophrenia. Neuropsychologia 45:107-23. [R-PB]

Rutter, M. (2005) Incidence of autism spectrum disorders: Changes over time and their meaning. Acta Paediatrica 94:2-15. [MCK]

Rzhetsky, A., Wajngurt, D., Park, N. \& Zheng T. (2007) Probing genetic overlap among complex human phenotypes. Proceedings of the National Academy of Sciences USA 104(28):11694-99. [GV]

Sacco, R., Militerni, R., Frolli, A., Bravaccio, C., Gritti, A., Elia, M., Curatolo, P., Manzi, B., Trillo, S., Lenti, C., Saccani, M., Schneider, C., Melmed, R., Reichelt, K. L., Pascucci, T., Puglisi-Allegra, S. \& Persico, A. M. (2007) Clinical, morphological, and biochemical correlates of head circumference in autism. Biological Psychiatry 62:1038-47. [aBC]

Sack, A. T., van de Ven, Vincent, G., Etschenberg, S., Schatz, D. \& Linden, D. E. J. (2005) Enhanced vividness of mental imagery as a trait marker of schizophrenia? Schizophrenia Bulletin 31:97-104. [aBC]

Sahoo, T., Peters, S. U., Madduri, N. S., Glaze, D. G., German, J. R., Bird, L. M., Barbieri-Welge, R., Bichell, T. J., Beaudet, A. L. \& Bacino, C. A. (2006) Microarray based comparative genomic hybridization testing in deletion 
bearing patients with Angelman syndrome: Genotype-phenotype correlations. Journal of Medical Genetics 43:512-16. [aBC]

Salazar-Fraile, J., Tabarés-Seisdedos, R., Selva-Vera, G., Balanzá-Martinez, V., Martínez-Aran, A., Catalán, J., Baldeweg, T., Vilela-Soler, C., Leal-Cercós, C., Vieta, E. \& Gomez-Beneyto, M. (2004) Recall and recognition confabulation in psychotic and bipolar disorders: Evidence for two different types without unitary mechanisms. Comprehensive Psychiatry 45:281-82. $[\mathrm{aBC}]$

Salem, J. E. \& Kring, A. M. (1998) The role of gender differences in the reduction of etiologic heterogeneity in schizophrenia. Clinical Psychology Review 18:795-819. [aBC]

Salovey, P. \& Mayer, J. D. (1990) Emotional intelligence. Imagination, Cognition and Personality 9:185-211. [rBC]

Samaco, R. C., Hogart, A. \& LaSalle, J. M. (2005) Epigenetic overlap in autismspectrum neurodevelopmental disorders: MECP2 deficiency causes reduced expression of UBE3A and GABRB3. Human Molecular Genetics 14:483-92. [arBC]

Samaco, R. C., Nagarajan, R. P., Braunschweig, D. \& LaSalle, J. M. (2004) Multiple pathways regulate MeCP2 expression in normal brain development and exhibit defects in autism-spectrum disorders. Human Molecular Genetics 13:629-39. [aBC]

Sander, D., Grafman, J. \& Zalla, T. (2003) The human amygdala: An evolved system for relevance detection. Reviews in the Neurosciences 14:306-16. [aBC]

Sanfilipo, M., Lafargue, T., Rusinek, H., Arena, L., Loneragan, C., Lautin, A., Feiner, D., Rotrosen, J. \& Wolkin, A. (2000) Volumetric measure of the frontal and temporal lobe regions in schizophrenia: Relationship to negative symptoms. Archives of General Psychiatry 57:471-80. [rBC]

Sarimski, K. (2003) Behavioural and emotional characteristics in children with Sotos syndrome and learning disabilities. Developmental Medicine and Child Neurology 45:172-78. [rBC]

(2004) Communicative competence and behavioural phenotype in children with Smith-Magenis syndrome. Genetic Counseling 15:347-55. [rBC]

Sass, L. A. (2001) Self and world in schizophrenia: Three classic approaches. Philosophy, Psychiatry and Psychology 8:252-70. [rBC]

Sass, L. A. \& Parnas, J. (2003) Schizophrenia, consciousness, and the self. Schizophrenia Bulletin 29:427-44. [aBC]

Saugstad, L. F. (1998) Cerebral lateralization and rate of maturation. International Journal of Psychophysiology 28:37-62. [aBC]

(1999) A lack of cerebral lateralization in schizophrenia is within the normal variation in brain maturation but indicates late, slow maturation. Schizophrenia Research 39:183-96. [aBC]

Saxena, A., Frank, D., Panichkul, P., Van den Veyver, I. B., Tycko, B. \& Thaker, H. (2003) The product of the imprinted gene IPL marks human villous cytotrophoblast and is lost in complete hydatidiform mole. Placenta $24: 835-42$. [aBC]

Schachar, R. J., Crosbie, J., Barr, C. L., Ornstein, T. J., Kennedy, J., Malone, M., Roberts, W., Ickowicz, A., Tannock, R., Chen, S. \& Pathare, T. (2005) Inhibition of motor responses in siblings concordant and discordant for attention deficit hyperactivity disorder. American Journal of Psychiatry 162(6):1076-82. [LMG]

Schanen, N. C. (2006) Epigenetics of autism spectrum disorders. Human Molecular Genetics 15(S2):r138-r150. [aBC]

Schiffman, J., Pestle, S., Mednick, S., Ekstrom, M., Sorensen, H. \& Mednick, S. (2005) Childhood laterality and adult schizophrenia spectrum disorders: A prospective investigation. Schizophrenia Research $72: 151-60 . \quad[\mathrm{aBC}]$

Schneider, F., Weiss, U., Kessler, C., Salloum, J. B., Posse, S., Grodd, W. \& MüllerGärtner, H. W. (1998) Differential amygdala activation in schizophrenia during sadness. Schizophrenia Research 34:133-42. [aBC]

Schneider, U., Borsutzky, M., Seifert, J., Leweke, F. M., Huber, T. J., Rollnik, J. D. \& Emrich, H. M. (2002) Reduced binocular depth inversion in schizophrenic patients. Schizophrenia Research 53:101-108. [aBC]

Schoenemann, P. T. (2006) Evolution of the size and functional areas of the human brain. Annual Review of Anthropology 35:379-406. [aBC]

Schoenemann, P. T., Sheehan, M. J. \& Glotzer, L. D. (2005) Prefrontal white matter volume is disproportionately larger in humans than in other primates. Nature Neuroscience 8:242-52. [aBC]

Scholten, M. R., van Honk, J., Aleman, A. \& Kahn, R. S. (2006) Behavioral inhibition system (BIS), behavioral activation system (BAS) and schizophrenia: Relationship with psychopathology and physiology. Journal of Psychiatric Research 40:638-45. [R-PB]

Schroer, R. J., Phelan, M. C., Michaelis, R. C., Crawford, E. C., Skinner, S. A., Cuccaro, M., Simensen, R. J., Bishop, J., Skinner, C., Fender, D. \& Stevenson, R. E. (1998) Autism and maternally derived aberrations of chromosome 15q. American Journal of Medical Genetics 76:327-36. [aBC]

Schultz, R. \& Klin, A. (2002) Genetics of childhood disorders: XLIII. Autism, part 2 Neural foundations. Journal of the American Academy of Child and Adolescent Psychiatry 41:1259-62. [R-PB]
Schumann, C. M., Hamstra, J., Goodlin-Jones, B. L., Lotspeich, L. J., Kwon, H., Buonocore, M. H., Lammers, C. R., Reiss, A. L. \& Amaral, D. G. (2004) The amygdala is enlarged in children but not adolescents with autism; the hippocampus is enlarged at all ages. Journal of Neuroscience 24:6392-401. [aBC]

Schürhoff, F., Laguerre, A., Szöke, A., Méary, A. \& Leboyer, M. (2005) Schizotypal dimensions: Continuity between schizophrenia and bipolar disorders. Schizophrenia Research 80:235-42. [aBC]

Schwartz, C. E., Wright, C. I., Shin, L. M., Kagan, J. \& Rauch, S. L. (2003a) Inhibited and uninhibited infants "grown up": Adult amygdalar response to novelty. Science 300:1952-53. [aBC]

Schwartz, C. E., Wright, C. I., Shin, L. M., Kagan, J., Whalen, P. J., McMullin, K. G. \& Rauch, S. L. (2003b) Differential amygdalar response to novel versus newly familiar neutral faces: A functional MRI probe developed for studying inhibited temperament. Biological Psychiatry 53:854-62. [aBC]

Seal, M. L., Aleman, A. \& McGuire, P. K. (2004) Compelling imagery, unanticipated speech and deceptive memory: Neurocognitive models of auditory verbal hallucinations in schizophrenia. Cognitive Neuropsychiatry 9:43-72. [aBC]

Sebat, J., Lakshmi, B., Malhotra, D., Troge, J., Lese-Martin, C., Walsh, T., Yamrom, B., Yoon, S., Krasnitz, A., Kendall, J., Leotta, A., Pai, D., Zhang, R., Lee, Y. H., Hicks, J., Spence, S. J., Lee, A. T., Puura, K., Lehtimäki, T., Ledbetter, D., Gregersen, P. K., Bregman, J., Sutcliffe, J. S., Jobanputra, V., Chung, W., Warburton, D., King, M. C., Skuse, D., Geschwind, D. H., Gilliam, T. C., Ye, K., \& Wigler, M. (2007) Strong association of de novo copy number mutations with autism. Science 316(5823):445-49. [THJB, CKD, MCK]

Sebat, J., Lakshmi, B., Troge, J., Alexander, J., Young, J., Lundin, P., Månér, S., Massa, H., Walker, M., Chi, M., Navin, N., Lucito, R., Healy, J., Hicks, J., Ye, K., Reiner, A., Gilliam, T. C., Trask, B., Patterson, N., Zetterberg, A., \& Wigler, M. (2004) Large-scale copy number polymorphism in the human genome. Science 305(5683):525-28. [CKD]

Seeman, P., Weinshenker, D., Quirion, R., Srivastava, L. K., Bhardwaj, S. K., Grandy, D. K., Premont, R. T., Sotnikova, T. D., Boksa, P., El-Ghundi, M., O’Dowd, B. F., George, S. R., Perreault, M. L., Männistö, P. T., Robinson, S., Palmiter, R. D. \& Tallerico, T. (2005) Dopamine supersensitivity correlates with D2High states, implying many paths to psychosis. Proceedings of the National Academy of Sciences of the United States of America 102:3513-18. [aBC]

Seidman, L. J., Thermenos, H. W., Poldrack, R. A., Peace, N. K., Koch, J. K., Faraone, S. V. \& Tsuang, M. T. (2006) Altered brain activation in dorsolateral prefrontal cortex in adolescents and young adults at genetic risk for schizophrenia: An fMRI study of working memory. Schizophrenia Research 85: $58-72 . \quad[\mathrm{aBC}]$

Seldon, H. L. (2005) Does brain white matter growth expand the cortex like a balloon? Hypothesis and consequences. Laterality 10:81-95. [aBC]

Senju, A., Tojo, Y., Dairoku, H. \& Hasegawa, T. (2004) Reflexive orienting in response to eye gaze and an arrow in children with and without autism. Journal of Child Psychology and Psychiatry, and Allied Disciplines 45:445-58. [rBC, RL]

Serajee, F. J., Nabi, R., Zhong, H. \& Mahbubul Huq, A. H. M. (2003) Association of INPP1:PIK3CG, and TSC2 gene variants with autistic disorder: Implications for phosphatidylinositol signalling in autism. Journal of Medical Genetics 40:e119. [aBC]

Serajee, F. J., Zhong, H. \& Mahbubul Huq, A. H. (2006) Association of Reelin gene polymorphisms with autism. Genomics 87:75-83. [rBC]

Shamay-Tsoory, S. G., Shur, S., Barcai-Goodman, L., Medlovich, S., Harari, H. \& Levkovitz, Y. (2007) Dissociation of cognitive from affective components of theory of mind in schizophrenia. Psychiatry Research 149:11-23. [aBC, AT]

Shao, Y., Cuccaro, M. L., Hauser, E. R., Raiford, K. L., Menold, M. M., Wolpert, C. M., Ravan, S. A., Elston, L., Decena, K., Donnelly, S. L., Abramson, R. K., Wright, H. H., DeLong, G. R., Gilbert, J. R. \& Pericak-Vance, M. A. (2003) Fine mapping of autistic disorder to chromosome 15q11-q13 by use of phenotypic subtypes. American Journal of Human Genetics 72(3):539-48. [LMG]

Sharma, R. P., Dowd, S. M. \& Janicak, P. G. (1999) Hallucinations in the acute schizophrenic-type psychosis: Effects of gender and age of illness onset. Schizophrenia Research 37:91-95. [aBC]

Shaw, J., Claridge, G. \& Clark, K. (2001) Schizotypy and the shift from dextrality: A study of handedness in a large non-clinical sample. Schizophrenia Research $50: 181-89 . \quad[\mathrm{aBC}]$

Shaw, P., Lawrence, E. J., Radbourne, C., Bramham, J., Polkey, C. E. \& David, A. S. (2004) The impact of early and late damage to the human amygdala on "theory of mind" reasoning. Brain 127:1535-48. [aBC]

Shaywitz, B. A., Shaywitz, S. E., Pugh, K. R., Constable, R. T., Skudlarski, P., Fulbright, R. K., Bronen, R. A., Fletcher, J. M., Shankweiler, D. P. \& Katz, L. (1995) Sex differences in the functional organization of the brain for language. Nature 373:607-609. [aBC]

Sheitman, B. B., Kraus, J. E., Bodfish, J. W. \& Carmel, H. (2004) Are the negative symptoms of schizophrenia consistent with an autistic spectrum illness? Schizophrenia Research 69:119-20. [aBC] 
Sherr, E. H., Owen, R., Albertson, D. G., Pinkel, D., Cotter, P. D., Slavotinek, A M., Hetts, S. W., Jeremy, R. J., Schilmoeller, G., Schilmoeller, K., Wakahiro, M. \& Barkovich, A. J. (2005) Genomic microarray analysis identifies candidate loci in patients with corpus callosum anomalies. Neurology 65:1496-98. $[\mathrm{aBC}]$

Shibayama, A., Cook, E. H., Feng, J., Glanzmann, C., Yan, J., Craddock, N., Jones, I. R., Goldman, D., Heston, L. L. \& Sommer, S. S. (2004) MECP2 structural and 3'-UTR variants in schizophrenia, autism and other psychiatric diseases: A possible association with autism. American Journal of Medical Genetics. Part B, Neuropsychiatric Genetics 128:50-53. [rBC]

Shifman, S., Johannesson, M., Bronstein, M., Chen, S. X., Collier, D. A., Craddock, N. J., Kendler, K. S., Li, T., O’Donovan, M., O’Neill, F. A., Owen, M. J., Walsh, D., Weinberger, D. R., Sun, C., Flint, J. \& Darvasi, A. (2008) Genome-wide association identifies a common variant in the reelin gene that increases the risk of schizophrenia only in women. PLoS Genetics 4:E28. [rBC]

Shirakawa, O., Kitamura, N., Lin, X. H., Hashimoto, T. \& Maeda, K. (2001) Abnormal neurochemical asymmetry in the temporal lobe of schizophrenia. Progress in Neuro-Psychopharmacology and Biological Psychiatry 25:867-77. [aBC]

Siekmeier, P. J. \& Hoffman, R. E. (2002) Enhanced semantic priming in schizophrenia: A computer model based on excessive pruning of local connections in association cortex. British Journal of Psychiatry 180:345 -50. [aBC, LMO]

Silberberg, N. E. \& Silberberg, M. C. (1967) Hyperlexia - Specific word recognition skills in young children. Exceptional Children 34:41-42. [aBC]

(1971) Hyperlexia - The other end of the spectrum. Journal of Special Education 5:233-42. $\quad[\mathrm{aBC}]$

Silk, J. B. (2007) Social components of fitness in primate groups. Science 317(5843): 1347-51. [LMG]

Silk, T. J., Rinehart, N., Bradshaw, J. L., Tonge, B., Egan, G., O’Boyle, M. W. \& Cunnington, R. (2006) Visuospatial processing and the function of prefrontalparietal networks in autism spectrum disorders: A functional MRI study. American Journal of Psychiatry 163:1440-43. [aBC]

Simon, T. J., Bearden, C. E., Moss, E. M., McDonald-McGinn, E. Z. \& Wang, P. P. (2002) Cognitive development in VCFS. Progress in Pediatric Cardiology 15:109-17. [aBC]

Simpson, J. L., de la Cruz, F., Swerdloff, R. S., Samango-Sprouse, C., Skakkebaek, N. E., Graham, J. M., Jr., Hassold, T., Aylstock, M., Meyer-Bahlburg, H. F., Willard, H. F., Hall, J. G., Salameh, W., Boone, K., Staessen, C., Geschwind, D., Giedd, J., Dobs, A. S., Rogol, A., Brinton, B. \& Paulsen, C. A. (2003) Klinefelter syndrome: Expanding the phenotype and identifying new research directions. Genetics in Medicine 5:460-68. [aBC]

Singer, T. (2006) The neuronal basis and ontogeny of empathy and mind reading Review of literature and implications for future research. Neuroscience and Biobehavioral Reviews 30:855-63. [AT]

Singh, M. K., Giles, L. L. \& Nasrallah, H. A. (2006) Pain insensitivity in schizophrenia: Trait or state marker? Journal of Psychiatric Practice 12:90-102. [aBC]

Sipos, A., Rasmussen, F., Harrison, G., Tynelius, P., Lewis, G., Leon, D. A. \& Gunnell, D. (2004) Paternal age and schizophrenia: A population based cohort study. British Medical Journal 329(7474):1070. [GV, THJB]

Skinner, B. F. (1948) "Superstition" in the pigeon. Journal of Experimental Psychology 38:168-72. [rBC]

Skuse, D. H. (2000) Imprinting, the X-chromosome, and the male brain: Explaining sex differences in the liability to autism. Pediatric Research 47:9-16. [rBC]

(2005) X-linked genes and mental functioning. Human Molecular Genetics 14(1):R27-32. [arBC]

(2007) Rethinking the nature of genetic vulnerability to autistic spectrum disorders. Trends in Genetics 23:387-95. [rBC]

Skuse, D. H., James, R. S., Bishop, D. V., Coppin, B., Dalton, P., Aamodt-Leeper, G., Bacarese-Hamilton, M., Creswell, C., McGurk, R. \& Jacobs, P. A. (1997) Evidence from Turner's syndrome of an imprinted X-linked locus affecting cognitive function. Nature $387: 705-708 . \quad$ [rBC]

Skuse, D. H., Morris, J. S. \& Dolan, R. J. (2005) Functional dissociation of amygdala-modulated arousal and cognitive appraisal, in Turner syndrome. Brain 128:2084-96. [aBC]

Skuse, D., Morris, J. \& Lawrence, K. (2003) The amygdala and development of the social brain. Annals of the New York Academy of Sciences 1008:91-101. [aBC]

Smalley, S. L. (1998) Autism and tuberous sclerosis. Journal of Autism and Developmental Disorders 28(5):407-14. [CKD]

Smith, A. C. M., Dykens, E. \& Greenberg, F. (1998) Behavioral phenotype of Smith-Magenis syndrome (del 17p11.2). American Journal of Medical Genetics $81: 179-85 . \quad[\mathrm{rBC}]$

Smith, A. C. M., Magenis, R. E. \& Elsea, S. H. (2005) Overview of Smith-Magenis syndrome. Journal of the Association of Genetic Technologists 31:163-67. $[\mathrm{rBC}]$

Smith, F. M., Garfield, A. S. \& Ward, A. (2006) Regulation of growth and metabolism by imprinted genes. Cytogenetic and Genome Research 113:279-91. [aBC]
Smith, S. D. (2007) Genes, language development, and language disorders. Mental Retardation and Developmental Disabilities Research Reviews 13:96-105 $[\mathrm{aBC}]$

Smits, G. \& Kelsey, G. (2006) Imprinting weaves its web. Developmental Cell 11:598-99. [rBC]

Soderstrom, H., Rastam, M. \& Gillberg, C. (2002) Temperament and character in adults with Asperger syndrome. Autism 6:287-97. [aBC]

Sommer, I. E., Ramsey, N. F. \& Kahn, R. S. (2001) Language lateralization in schizophrenia, an fMRI study. Schizophrenia Research 52:57-67. [aBC]

Soni, S., Whittington, J., Holland, A. J., Webb, T., Maina, E., Boer, H. \& Clarke, D. (2007) The course and outcome of psychiatric illness in people with Prader-Willi syndrome: Implications for management and treatment. Journal of Intellectual Disability Research 51:32-42. [aBC]

(2008) The phenomenology and diagnosis of psychiatric illness in people with Prader-Willi syndrome. Psychological Medicine 51:32-42. [rBC]

Sorensen, H. J., Mortensen, E. L., Parnas, J. \& Mednick, S. A. (2006) Premorbid neurocognitive functioning in schizophrenia spectrum disorder. Schizophrenia Bulletin 32:578-83. [aBC]

Sparks, B. F., Friedman, S. D., Shaw, D. W., Aylward, E. H., Echelard, D., Artru, A A., Maravilla, K. R., Giedd, J. N., Munson, J., Dawson, G. \& Dager, S. R. (2002) Brain structural abnormalities in young children with autism spectrum disorder. Neurology 59:184-92. [R-PB]

Spence, S. J., Cantor, R. M., Chung, L., Kim, S., Geschwind, D. H., Alarcón, M. (2006) Stratification based on language-related endophenotypes in autism: Attempt to replicate reported linkage. American Journal of Medical Genetics, Part B: Neuropsychiatric Genetics 141(6):591-98. [CKD]

Sporn, A. L., Addington, A. M., Gogtay, N., Ordoñez, A. E., Gornick, M., Clasen, L., Greenstein, D., Tossell, J. W., Gochman, P., Lenane, M., Sharp, W. S., Straub, R. E. \& Rapoport, J. L. (2004a) Pervasive developmental disorder and childhood-onset schizophrenia: Comorbid disorder or a phenotypic variant of a very early onset illness? Biological Psychiatry 55:989-94. [aBC]

Sporn, A. L., Addington, A. M., Gornick, M., Kwon, L., Gogtay, N., Clasen, L. S., Greenstein, D., Ordonez, A. E., Bobb, A. J., Lenane, M. C., Gochman, P. A., Straub, R. E. \& Rapoport, J. L. (2004b) ATP10C and UBE3A genes in childhood onset schizophrenia. Biological Psychiatry 55:s171. [rBC]

Sprong, M., Schothorst, P., Vos, E., Hox, J. \& van Engeland, H. (2007) Theory of mind in schizophrenia: Meta-analysis. British Journal of Psychiatry 191:5-13. [rBC, SvR]

Srinivasan, T. N. \& Padmavati, R. (1997) Fertility and schizophrenia: Evidence for increased fertility in the relatives of schizophrenic patients. Acta Psychiatrica Scandinavica 96:260-64. [aBC, MCK]

Sritharan, A., Line, P., Sergejew, A., Silberstein, R., Egan, G. \& Copolov, D. (2005) EEG coherence measures during auditory hallucinations in schizophrenia. Psychiatry Research 136:189-200. [aBC]

Stahlberg, O., Soderstrom, H., Rastam, M. \& Gillberg, C. (2004) Bipolar disorder, schizophrenia, and other psychotic disorders in adults with childhood onset AD/HD and/or autism spectrum disorders. Journal of Neural Transmission 111:891-902. [aBC]

Stanfield, A. C., McIntosh, A. M., Spencer, M. D., Philip, R., Gaur, S. \& Lawrie, S. M. (in press) Towards a neuroanatomy of autism: A systematic review and meta-analysis of structural magnetic resonance imaging studies. European Psychiatry. $\quad[\mathrm{aBC}]$

Steiner, J. (2004) Gaze, dominance and humiliation in the Schreber case. International Journal of Psycho-Analysis 85:269-84. [aBC]

Stevenson, J. C., Everson, P. M., Williams, D. C., Hipskind, G., Grimes, M. \& Mahoney, E. R. (2007) Attention deficit/hyperactivity disorder (ADHD) symptoms and digit ratios in a college sample. American Journal of Human Biology 19:41-50. [MV]

Stopkova, P., Saito, T., Papolos, D. F., Vevera, J., Paclt, I., Zukov, I., Bersson, Y. B., Margolis, B. A., Strous, R. D. \& Lachman, H. M. (2004) Identification of PIK3C3 promoter variant associated with bipolar disorder and schizophrenia. Biological Psychiatry 55:981-88. [aBC]

Strelets, V. B., Novototsky-Vlasov, V. Y. \& Golikova, J. V. (2002) Cortical connectivity in high frequency beta-rhythm in schizophrenics with positive and negative symptoms. International Journal of Psychophysiology 44:101-15. [aBC]
[a.

Sugayama, S. M., Leone, C., Chauffaille, M. de L., Okay, T. S. \& Kim, C. A. (2007) Williams syndrome: Development of a new scoring system for clinical diagnosis. Clinics 62:159-66. [rBC]

Sugie, Y., Sugie, H., Fukuda, T. \& Ito, M. (2005) Neonatal factors in infants with autistic disorder and typically developing infants. Autism 9:487-94. $[\mathrm{aBC}]$

Sullivan, R. J. \& Allen, J. S. (1999) Social deficits associated with schizophrenia defined in terms of interpersonal Machiavellianism. Acta Psychiatrica Scandinavica 99:148-54. [aBC]

Sumich, A., Chitnis, X. A., Fannon, D. G., O'Ceallaigh, S., Doku, V. C., Faldrowicz, A. \& Sharma, T. (2005) Unreality symptoms and volumetric measures of Heschl's gyrus and planum temporal in first-episode psychosis. Biological Psychiatry 57:947-50. [aBC, R-PB] 
Sun, T., Patoine, C., Abu-Khalil, A., Visvader, J., Sum, E., Cherry, T. J., Orkin, S. H., Geschwind, D. H. \& Walsh, C. A. (2005) Early asymmetry of gene transcription in embryonic human left and right cerebral cortex. Science 308:1794-98. [aBC]

Surendran, S., Tyring, S. K. \& Matalon, R. (2005) Expression of calpastatin, minopontin, NIPSNAP1, rabaptin-5 and neuronatin in the phenylketonuria (PKU mouse brain: Possible role on cognitive defect seen in PKU. Neurochemistry International 46:595-99. [rBC]

Surguladze, S., Russell, T., Kucharska-Pietura, K., Travis, M. J., Giampietro, V., David, A. S. \& Phillips, M. L. (2006) A reversal of the normal pattern of parahippocampal response to neutral and fearful faces is associated with reality distortion in schizophrenia. Biological Psychiatry 60:423-31. [R-PB]

Sutcliffe, J. S., Delahanty, R. J., Prasad, H. C., McCauley, J. L., Han, Q., Jiang, L., Li, C., Folstein, S. E. \& Blakely, R. D. (2005) Allelic heterogeneity at the serotonin transporter locus (SLC6A4) confers susceptibility to autism and rigid-compulsive behaviors. American Journal of Human Genetics 77:265-79. [MCK]

Sutton, S. K., Burnette, C. P., Mundy, P. C., Meyer, J., Vaughan, A., Sanders, C. \& Yale, M. (2005) Resting cortical brain activity and social behavior in higher functioning children with autism. Journal of Child Psychology and Psychiatry 46:211-22. [aBC]

Suzuki, M., Zhou, S. Y., Takahashi, T., Hagino, H., Kawasaki, Y., Niu, L., Matsui, M., Seto, H. \& Kurachi, M. (2005) Differential contributions of prefrontal and temporolimbic pathology to mechanisms of psychosis. Brain 128:2109-22. $[\mathrm{aBC}]$

Svensson, A. C., Lichtenstein, P., Sandin, S. \& Hultman, C. M. (2007) Fertility of first-degree relatives of patients with schizophrenia: A three generation perspective. Schizophrenia Research 91:238-45. [MCK]

Svensson, A. C., Pawitan, Y., Cnattingius, S., Reilly, M. \& Lichtenstein, P. (2006) Familial aggregation of small-for-gestational-age births: The importance of fetal genetic effects. American Journal of Obstetrics and Gynecology 194:475-79. $\quad[\mathrm{aBC}]$

Swettenham, J., Condie, S., Campbell, R., Milne, E. \& Coleman, M. (2003) Does the perception of moving eyes trigger reflexive visual orienting in autism? In: Autism: Mind and brain, ed. U. Frith \& E. Hill, pp. 89-107. Oxford University Press. [RL]

Tabuchi, K., Blundell, J., Etherton, M. R., Hammer, R. E., Liu, X., Powell, C. M. \& Sudhof, T. C. (2007) A neuroligin-3 mutation implicated in autism increases inhibitory synaptic transmission in mice. Science 318:71-6. [aBC]

Tager-Flusberg, H., Joseph, R. \& Folstein, S. (2001) Current directions in research on autism. Mental Retardation and Developmental Disabilities Research Reviews 7:21-29. [R-PB]

Takarae, Y., Minshew, N. J., Luna, B. \& Sweeney, J. A. (2007) Atypical involvement of frontostriatal systems during sensorimotor control in autism. Psychiatry Research 156:117-27. [MKB]

Tallal, P., Ross, R. \& Curtiss, S. (1989) Unexpected sex-ratios in families of language/learning-impaired children. Neuropsychologia 27:987-98. [SK]

Tamimi, R. M., Lagiou, P., Mucci, L. A., Hsieh, C. C., Adami, H. O. \& Trichopoulos, D. (2003) Average energy intake among pregnant women carrying a boy compared with a girl. British Medical Journal 326:1245-46. [aBC]

Tamminga, C. A. \& Holcomb, H. H. (2005) Phenotype of schizophrenia: A review and formulation. Molecular Psychiatry 10:27-39. [aBC]

Tantam, D. (1988) Asperger's syndrome. Journal of Child Psychology and Psychiatry 29:245-55. [ST]

Tassabehji, M. (2003) Williams-Beuren syndrome: A challenge for genotypephenotype correlations. Human Molecular Genetics 12(S2):R229-37. [rBC]

Taylor, K. I., Zäch, P. \& Brugger, P. (2002) Why is magical ideation related to leftward deviation on an implicit line bisection task? Cortex 38:247-52. $[\mathrm{aBC}]$

Taylor, S. E. \& Brown, J. D. (1988) Illusion and well-being: A social psychological perspective on mental health. Psychological Bulletin 103:193-210. [ [rBC]

Temple, C. M. \& Carney, R. (1996) Reading skills in children with Turner's syndrome: An analysis of hyperplexia. Cortex 32:335-45. [rBC]

Temple, I. K. \& Shield, J. P. H. (2002) Transient neonatal diabetes, a disorder of imprinting. Journal of Medical Genetics 39:872-75. [rBC, BJAD]

Terracciano, A., Chiurazzi, P. \& Neri, G. (2005) Fragile X syndrome. American Journal of Medical Genetics. Part C, Seminars in Medical Genetics 137:32-37. [rBC]

Thoma, P. \& Daum, I. (2006) Neurocognitive mechanisms of figurative language processing - Evidence from clinical dysfunctions. Neuroscience and Biobehavioral Reviews 30:1182-1205. [aBC]

Tierney, E., Nwokoro, N. A., Porter, F. D., Freund, L. S., Ghuman, J. K. \& Kelley, R. (2001) Behavior phenotype in the RSH/Smith-Lemli-Opitz syndrome. American Journal of Medical Genetics 98(2):191-200. [CKD]

Toichi, M. \& Kamio, Y. (2002) Long-term memory and levels-of-processing in autism. Neuropsychologia 40:964-69. [aBC]
Toichi, M., Kamio, Y., Okada, T., Sakihama, M., Youngstrom, E. A., Findling, R. L. \& Yamamoto, K. (2002) A lack of self-consciousness in autism. American Journal of Psychiatry 159:1422-24. [aBC]

Tomasello, M., Carpenter, M., Call, J., Behne, T. \& Moll, H. (2005) Understanding and sharing intentions: The origins of cultural cognition. Behavioral and Brain Sciences 28:675-735. $\quad[\mathrm{aBC}]$

Tordjman, S., Anderson, G., Macbride, A., Hetzig, M., Snow, M., Hall, L., Ferrari, P. \& Cohen, D. J. (1997) Plasma B. endorphin, adrenocorticotropin hormone and cortisol in autism. Journal of Child Psychology and Psychiatry 38(6):705-16. [ST]

Tordjman, S., Antoine, C., Cohen, D. J., Gauvain-Piquard, A., Carlier, M., Roubertoux, P. L. \& Ferrari, P. (1999) Etude des conduites autoagressives, de la réactivité à la douleur et de leurs interrelations chez les enfants autistes [Study of the relationships between self-injurious behavior and pain reactivity in infantile autism]. L’Encéphale 25:122-34. [ST]

Tordjman, S., Drapier, D., Bonnot, O., Graignic, R., Fortes, S., Cohen, D., Millet, B., Laurent, C. \& Roubertoux, P. L. (2007) Animal models relevant to schizophrenia and autism: Validity and limitations. Behavior Genetics 37(1):61-78. [ST]

Tordjman, S., Gutknecht, L., Carlier, M., Spitz, E., Antoine, C., Slama, F., Carsalade, V., Cohen, D. J., Ferrari, P., Roubertoux, P. L. \& Anderson, G. M. (2001) Role of the serotonin transporter gene in the behavioral expression of autism. Molecular Psychiatry 6:434-39. [ST]

Torres, A. R., Maciulis, A., Stubbs, E. G., Cutler, A. \& Odell, D. (2002) The transmission disequilibrium test suggests that HLA-DR4 and DR13 are linked to autism spectrum disorder. Human Immunology 63:311-16. $[\mathrm{rBC}]$

Toulopoulou, T., Mapua-Filbey, F., Quraishi, S., Kravariti, E., Morris, R. G., McDonald, C., Walshe, M., Bramon, E. \& Murray, R. M. (2005) Cognitive performance in presumed obligate carriers for psychosis. British Journal of Psychiatry 187:284-85. [aBC]

Townsend, J., Courchesne, E., Singer-Harris, N., Covington, J., Westerfield, M., Lyden, P., Lowry, T. P. \& Press, G. A. (1999) Spatial attention deficits in patients with acquired or developmental cerebellar abnormality. Journal of Neuroscience 19:5632-43. [R-PB]

Treffert, D. A. (2000) Extraordinary people: Understanding savant syndrome. iUniverse.com. $[\mathrm{rBC}]$

Trémeau, F., Malaspina, D., Duval, F., Corrêa, H., Hager-Budny, M., Coin-Bariou, L., Macher, J. \& Gorman, J. M. (2005) Facial expressiveness in patients with schizophrenia compared to depressed patients and nonpatient comparison subjects. American Journal of Psychiatry 162:92-101. [aBC]

Trillingsgaard, A. \& Østergaard, J. R. (2004) Autism in Angelman syndrome: An exploration of comorbidity. Autism 8:163-74. [aBC]

Troisi, A. (2005) The concept of alternative strategies and its relevance to psychiatry and clinical psychology. Neuroscience and Biobehavioral Reviews 29:15968. $[\mathrm{AT}]$

Troisi, A., Pasini, A. \& Spalletta, G. (2001) Season of birth, gender and negative symptoms in schizophrenia. European Psychiatry 16:342-48. [aBC]

Troisi, A., Pompili, E., Binello, L. \& Sterpone, A. (2007) Facial expressivity during the clinical interview as a predictor functional disability in schizophrenia: A pilot study. Progress in Neuro-Psychopharmacology and Biological Psychiatry 31:475-81. [aBC]

Tsai, S. J. (2005) Is autism caused by early hyperactivity of brain-derived neurotrophic factor? Medical Hypotheses 65:79-82. [aBC]

Tsakanikos, E. \& Claridge, G. (2005) More words, less words: Verbal fluency as a function of "positive" and "negative" schizotypy. Personality and Individual Differences 39:705-13. [aBC]

Tsuang, M. T., Taylor, L. \& Faraone, S. V. (2004) An overview of the genetics of psychotic mood disorders. Journal of Psychiatric Research 38:3-15. [aBC]

Tuncer, M. C., Hatipoglu, E. S. \& Ozates, M. (2005) Sexual dimorphism and handedness in the human corpus callosum based on magnetic resonance imaging. Surgical and Radiologic Anatomy 27:254-59. [aBC]

Turetsky, B., Cowell, P. E., Gur, R. C., Grossman, R. I., Shtasel, D. L. \& Gur, R. E. (1995) Frontal and temporal lobe brain volumes in schizophrenia. Relationship to symptoms and clinical subtype. Archives of General Psychiatry 52:1061-70. [rBC]

Turkeltaub, P. E., Flowers, D. L., Verbalis, A., Miranda, M., Gareau, L. \& Eden, G. F. (2004) The neural basis of hyperlexic reading: An fMRI case study. Neuron $41: 11-25 . \quad[\mathrm{aBC}]$

Turner, M. A. (1999) Generating novel ideas: Fluency performance in high-functioning and learning disabled individuals with autism. Journal of Child Psychology and Psychiatry, and Allied Disciplines 40:189-201. [aBC]

Tycko, B. \& Morison, I. M. (2002) Physiological functions of imprinted genes. Journal of Cellular Physiology 192:245-58. [aBC]

Uhlhaas, P. J., Phillips, W. A., Schenkel, L. S. \& Silverstein, S. M. (2006) Theory of mind and perceptual context-processing in schizophrenia. Cognitive Neuropsychiatry 11:416-36. [MKB] 
Uhlhaas, P. J. \& Silverstein, S. M. (2005) Perceptual organization in schizophrenia spectrum disorders: A review of empirical research and associated theories. Psychological Bulletin 131:618-32. [MKB]

Valenza, E., Simion, F., Cassia, V. M. \& Umilta, C. (1996) Face preference at birth. Journal of Experimental Psychology. Human Perception and Performance 22:892-903. [R-PB]

van Daalen, E., Swinkels, S. H. N., Dietz, C., van Engeland, H. \& Buitelaar, J. K. (2007) Body length and head growth in the first year of life in autism. Pediatric Neurology 37:324-30. [rBC]

Van Den Bogaert, A., Del-Favero, J. \& Van Broeckhoven, C. (2006) Major affective disorders and schizophrenia: A common molecular signature? Human Mutation 27:833-53. [aBC]

Van Den Bosch, R. J., Van Asma, M. J., Rombouts, R. \& Louwerens, J. W. (1992) Coping style and cognitive dysfunction in schizophrenic patients. British Journal of Psychiatry Supplement 18:123-28. [ST]

van Elst, L. T. \& Trimble, M. R. (2003) Amygdala pathology in schizophrenia and psychosis of epilepsy. Current Opinion in Psychiatry 16:321-26. [aBC]

Van Engeland, H. \& Van Der Gaag, R. J. (1994) MCDD in childhood: A precursor of schizophrenic spectrum disorders. Schizophrenia Research 11:197. [ST]

van Rijn, S., Aleman, A., Swaab, H. \& Kahn, R. S. (2005) Neurobiology of emotion and high risk for schizophrenia: Role of the amygdala and the X-chromosome. Neuroscience and Biobehavioral Reviews 29:385-97. [arBC]

(2006a) Klinefelter's syndrome (karyotype 47,XXY) and schizophrenia-spectrum pathology. British Journal of Psychiatry 189(5):459-61. [SvR]

van Rijn, S., Aleman, A., Swaab, H., Krijn, T., Vingerhoets, G. \& Kahn, R. (2007) What is said versus how it is said: Comprehension of affective prosody in men with Klinefelter (47,XXY) syndrome. Journal of the International Neuropsychological Society 13(6):1065-70. [SvR]

(in press) Social behavior and autism traits in a sex chromosomal disorder: Klinefelter $(47, \mathrm{XXY})$ syndrome. Journal of Autism and Developmental Disorders. [SvR]

van Rijn, S., Swaab, H., Aleman, A. \& Kahn, R. S. (2006b) X chromosomal effects on social cognitive processing and emotion regulation: A study with Klinefelter men $(47, \mathrm{XXY})$. Schizophrenia Research 84(2-3):194-203. [SvR]

Varrault, A., Gueydan, C., Delalbre, A., Bellmann, A., Houssami, S., Aknin, C., Severac, D., Chotard, L., Kahli, M., Le Digarcher, A., Pavlidis, P. \& Journot, L. (2006) Zacl regulates an imprinted gene network critically involved in the control of embryonic growth. Developmental Cell 11:711-22. [rBC]

Vawter, M. P., Harvey, P. D. \& Delisi, L. E. (2007) Dysregulation of X-linked gene expression in Klinefelter's syndrome and association with verbal cognition. American Journal of Medical Genetics. Part B. Neuropsychiatric Genetics 144(B):728-34. [rBC]

Veltman, M. W., Craig, E. E. \& Bolton, P. F. (2005) Autism spectrum disorders in Prader-Willi and Angelman syndromes: A systematic review. Psychiatric Genetics 15:243-54. [aBC, DH]

Veltman, M. W., Thompson, R. J., Roberts, S. E., Thomas, N. S., Whittington, J. \& Bolton, P. F. (2004) Prader-Willi syndrome - A study comparing deletion and uniparental disomy cases with reference to autism spectrum disorders. European Child and Adolescent Psychiatry 13:42-50. [aBC]

Venables, P. H. \& Bailes, K. (1994) The structure of schizotypy, its relation to subdiagnoses of schizophrenia and to sex and age. British Journal of Clinical Psychology 33(3):277-94. [aBC]

Venkatasubramanian, G., Chittiprol, S., Neelakantachar, N., Naveen, M. N., Thirthall, J., Gangadhar, B. N. \& Shetty, K. T. (2007) Insulin and insulin-like growth factor-1 abnormalities in antipsychotic-naive schizophrenia. American Journal of Psychiatry 164(10):1557-1660. [GV]

Venkatasubramanian, G., Jayakumar, P. N., Gangadhar, B. N., Janakiramaiah, N., Subbakrishna, D. K. \& Keshavan, M. S. (2002) Never-treated, younger onset schizophrenia patients have smaller corpus callosum. Biological Psychiatry 51:28S. (abstract). [GV]

Vercammen, A., de Haan, E. H. \& Aleman, A. (in press) Hearing a voice in the noise: Auditory hallucinations and speech perception. Psychological Medicine. [TV]

Verdoux, H., Liraud, F., Droulout, T., Theillay, G., Parrot, M. \& Franck, N. (2004) Is the intensity of Schneiderian symptoms related to handedness and speech disorder in subjects with psychosis? Schizophrenia Research 67:167-73. $[\mathrm{aBC}]$

Verhoeven, W. M., Tuinier, S. \& Curfs, L. M. (2003) Prader-Willi syndrome: The psychopathological phenotype in uniparental disomy. Journal of Medical Genetics 40:E112. [aBC]

Vermeulen, P. (2001) Autistic thinking - This is the title. Jessica Kingsley. [aBC]

Vicari, S., Bates, E., Caselli, M. C., Pasqualetti, P., Gagliardi, C., Tonucci, F. \& Volterra, V. (2004) Neuropsychological profile of Italians with Williams syndrome: An example of a dissociation between language and cognition? Journal of the International Neuropsychological Society 10:862-76. [rBC]

Vidal, C. N., Nicolson, R., Devito, T. J., Hayashi, K. M., Geaga, J. A., Drost, D. J., Williamson, P. C., Rajakumar, N., Sui, Y., Dutton, R. A., Toga, A. W. \&
Thompson, P. M. (2006) Mapping corpus callosum deficits in autism: An index of aberrant cortical connectivity. Biological Psychiatry 60:218-25. [aBC]

Vogels, A., De Hert, M., Descheemaeker, M. J., Govers, V., Devriendt, K., Legius, E., Prinzie, P. \& Fryns, J. P. (2004) Psychotic disorders in Prader-Willi syndrome. American Journal of Medical Genetics: Part A 127:238-43. [aBC]

Vogels, A., Matthijs, G., Legius, E., Devriendt, K. \& Fryns, J. P. (2003) Chromosome 15 maternal uniparental disomy and psychosis in Prader-Willi syndrome. Journal of Medical Genetics 40:72-73. [aBC]

Voight, B. F., Kudaravalli, S., Wen, X. \& Pritchard, J. K. (2006) A map of recent positive selection in the human genome. PLoS Biology 4:e72. [aBC]

Volkmar, F. R. \& Cohen, D. J. (1991) Comorbid association of autism and schizophrenia. American Journal of Psychiatry 148:1705-707. [aBC]

Vollema, M. G., Sitskoorn, M. M., Appels, M. C. \& Kahn, R. S. (2002) Does the Schizotypal Personality Questionnaire reflect the biological-genetic vulnerability to schizophrenia? Schizophrenia Research 54:39-45. [aBC]

von Károlyi, C., Winner, E., Gray, W. \& Sherman, G. F. (2003) Dyslexia linked to talent: Global visual-spatial ability. Brain and Language 85:427-31. [aBC]

Voracek, M. \& Dressler, S. G. (2006) Lack of correlation between digit ratio (2D:4D) and Baron-Cohen's "Reading the Mind in the Eyes" test, empathy, systemising, and autism-spectrum quotients in a general population sample. Personality and Individual Differences 41:1481-91. [MV]

(2007) Digit ratio (2D:4D) in twins: Heritability estimates and evidence for a masculinized trait expression in women from opposite-sex pairs. Psychological Reports 100:115-26. [MV]

Voracek, M., Dressler, S. G. \& Manning, J. T. (2007) Evidence for assortative mating on digit ratio (2D:4D), a biomarker for prenatal androgen exposure. Journal of Biosocial Science 39:599-612. [MV]

Voracek, M. \& Offenmüller, D. (2007) Digit ratios (2D:4D and other) and relative thumb length: A test of developmental stability. Perceptual and Motor Skills 105:143-52. [MV]

Voracek, M. \& Pavlovic, S. (2007) The tell-tale hand: The relationship of 2D:4D to perceived attractiveness, sex typicality, and other attributes of palms. Journal of Individual Differences 28:88-97. [MV]

Voracek, M., Reimer, B., Ertl, C. \& Dressler, S. G. (2006) Digit ratio (2D:4D), lateral preferences, and performance in fencing. Perceptual and Motor Skills 103:427-46. [MV]

Vorstman, J. A. S., Morcus, M. E. J., Duijff, S. N., Klaassen, P. W. J., Heineman-de Boer, J. A., Beemer, F. A., Swaab, H., Kahn, R. S. \& van Engeland, H. (2006) The $22 q 11.2$ deletion in children: High rate of autistic disorders and early onset of psychotic symptoms. Journal of the American Academy of Child and Adolescent Psychiatry 45(9):1104-13. [THJB, aBC]

Vourdas, A., Pipe, R., Corrigall, R. \& Frangou, S. (2003) Increased developmental deviance and premorbid dysfunction in early onset schizophrenia. Schizophrenia Research 62:13-22. [aBC]

Vygotsky, L. S. (1962) Thought and language. MIT Press. [rBC]

Waddington, J. L. \& Youssef, H. A. (1996) Familial-genetic and reproductive epidemiology of schizophrenia in rural Ireland: Age at onset, familial morbid risk and parental fertility. Acta Psychiatrica Scandinavica 93:62-68. [aBC, MCK]

Wahlbeck, K., Forsén, T., Osmond, C., Barker, D. J. \& Eriksson, J. G. (2001a) Association of schizophrenia with low maternal body mass index, small size at birth, and thinness during childhood. Archives of General Psychiatry 58:48-52. [aBC]

Wahlbeck, K., Osmond, C., Forsén, T., Barker, D. J. \& Eriksson, J. G. (2001b) Associations between childhood living circumstances and schizophrenia: A population-based cohort study. Acta Psychiatrica Scandinavica 104:356-60. [aBC]

Waiter, G. D., Williams, J. H. G., Murray, A. D., Gilchrist, A., Perrett, D. I. \& Whiten, A. (2005) Structural white matter deficits in high-functioning individuals with autistic spectrum disorder: A voxel-based investigation. NeuroImage 24:455-61. [aBC, GV]

Walder, D. J., Andersson, T. L. C., McMillan, A. L., Breedlove, S. M. \& Walker, E. F. (2006a) Sex differences in digit ratio (2D:4D) are disrupted in adolescents with schizotypal personality disorder: Altered prenatal gonadal hormone levels as a risk factor. Schizophrenia Research 86:118-22. [aBC, MV]

Walder, D. J., Seidman, L. J., Cullen, N., Su, J., Tsuang, M. T. \& Goldstein, J. M. (2006b) Sex differences in language dysfunction in schizophrenia. American Journal of Psychiatry 163:470-77. [aBC]

Walker, E. \& Bollini, A. M. (2002) Pubertal neurodevelopment and the emergence of psychotic symptoms. Schizophrenia Research 54:17-23. [aBC]

Walston, F., Blennerhassett, R. C. \& Charlton, B. G. (2000) Theory of mind, persecutory delusions and the somatic marker mechanism. Cognitive Neuropsychiatry 5:161-74. [KNT]

Wassink, T. H., Brzustowicz, L. M., Bartlett, C. W., Szatmari, P. (2004) The search for autism disease genes. Mental Retardation and Developmental Disabilities Research Reviews 10(4):272-83. [CKD]

Watson, S. J., Akil, H., Berger, P. A. \& Barchas, J. D. (1979) Some observations on the opiate peptides and schizophrenia. Archives of General Psychiatry $36: 35-41$. [ST] 
Weaver, I. C. G., Cervoni, N., Champagne, F. A., D’Alessio, A. C., Sharma, S., Seckl, J. R., Dymov, S., Szyf, M. \& Meaney, M. J. (2004) Epigenetic programming by maternal behavior. Nature Neuroscience 7:847-54. [BJAD]

Webb, S. J., Nalty, T., Munson, J., Brock, C., Abbott, R. \& Dawson, G. (2007) Rate of head circumference growth as a function of autism diagnosis and history of autistic regression. Journal of Child Neurology 22:1182-90. [rBC]

Webster, J. P. (2007) The effect of Toxoplasma gondii on animal behavior: playing cat and mouse. Schizophrenia Bulletin 33:752-56. [rBC]

Webster, J. P., Lamberton, P. H. L., Donnelly, C. A. \& Torrey, E. F. (2006) Parasites as causative agents of human affective disorders? The impact of anti-psychotic, mood-stabilizer and anti-parasite medication on Toxoplasma gondii's ability to alter host behaviour. Proceedings of the Royal Society of London Series B, Biological Sciences 273:1023-30. [rBC]

Weickert, C. S., Hyde, T. M., Lipska, B. K., Herman, M. M., Weinberger, D. R. \& Kleinman, J. E. (2003) Reduced brain-derived neurotrophic factor in prefrontal cortex of patients with schizophrenia. Molecular Psychiatry 8:592-610. [aBC, LMO]

Weickert, C. S., Ligons, D. L., Romanczyk, T., Ungaro, G., Hyde, T. M., Herman, M. M., Weinberger, D. R. \& Kleinman, J. E. (2005) Reductions in neurotrophin receptor mRNAs in the prefrontal cortex of patients with schizophrenia Molecular Psychiatry 10:637-50. [aBC]

Weinzimer, S. A., McDonald-McGinn, D. M., Driscoll, D. A., Emanuel, B. S., Zackai, E. H. \& Moshang, T. (1998) Growth hormone deficiency in patients with 22q11.2 deletion: Expanding the phenotype. Pediatrics 101:929-32. $[\mathrm{rBC}]$

Weiss, E. M., Hofer, A., Golaszewski, S., Siedentopf, C., Felber, S. \& Fleischhacker, W. W. (2006) Language lateralization in unmedicated patients during an acute episode of schizophrenia: A functional MRI study. Psychiatry Research 146:185-90. [aBC]

Weißegger, V. (2004) Explorative KFA zur Beschreibung von neurobiologischen Typen mittels konsistentem und inkonsistentem zerebralen Dominanzmuster und dem Fingerlängenverhältnis [Explorative configuration frequency analysis (CFA) for the description of neurobiological types based on consistent and inconsistent cerebral dominance patterns and finger-length ratio.] Unpublished master's thesis, University of Graz, Austria. [MV]

Westergaard, T., Mortensen, P. B., Pedersen, C. B., Wohlfahrt, J. \& Melbye, M. (1999) Exposure to prenatal and childhood infections and the risk of schizophrenia: Suggestions from a study of sibship characteristics and influenza prevalence. Archives of General Psychiatry 56:993-98. [aBC]

(2001) Sibships characteristics, influenza and risk of schizophrenia. A population-based cohort study. Ugeskrift for Laeger (Weekly Journal for Doctors) 163:4745-49. [aBC]

Whalen, P. J., Kagan, J., Cook, R. G., Davis, F. C., Kim, H., Polis, S., McLaren, D G., Somerville, L. H., McLean, A. A., Maxwell, J. S. \& Johnstone, T. (2004) Human amygdala responsivity to masked fearful eye whites. Science 306:2061. [aBC]

Whalley, H. C., Simonotto, E., Flett, S., Marshall, I., Ebmeier, K. P., Owens, D. G. C., Goddard, N. H., Johnstone, E. C. \& Lawrie, S. M. (2004) fMRI correlates of state and trait effects in subjects at genetically enhanced risk of schizophrenia. Brain 127:478-90. [aBC]

Whalley, H. C., Simonotto, E., Marshall, I., Owens, D. G. C., Goddard, N. H., Johnstone, E. C. \& Lawrie, S. M. (2005) Functional disconnectivity in subjects at high genetic risk of schizophrenia. Brain 128:2097-2108. [aBC]

Wheelwright, S. \& Baron-Cohen, S. (2001) The link between autism and skills such as engineering, maths, physics and computing: A reply to Jarrold and Routh. Autism 5:223-27. [arBC]

Whitehouse, A., Maybery, M. T. \& Durkin, K. (2006) Inner speech impairments in autism. Journal of Child Psychology and Psychiatry 47:857-65. $[\mathrm{aBC}, \mathrm{WF}]$

Whittington, J. \& Holland, T. (2004) Prader-Willi syndrome: Development and manifestations. Cambridge University Press. [arBC]

Whittington, J., Holland, A., Webb, T., Butler, J., Clarke, D. \& Boer, H. (2004) Cognitive abilities and genotype in a population-based sample of people with Prader-Willi syndrome. Journal of Intellectual Disability Research 48:172-87. [aBC]

World Health Organisation (2007) World Health Organisation Core Health Indicators Database. Available at: http://www.who.int/whosis/database/ core/core_select.cfm [DN]

Wible, C. G., Anderson, J., Shenton, M. E., Kricun, A., Hirayasu, Y., Tanaka, S., Levitt, J. J., O’Donnell, B. F., Kikinis, R., Jolesz, F. A. \& McCarley, R. W. (2001) Prefrontal cortex, negative symptoms, and schizophrenia: An MRI study. Psychiatry Research 108:65-78. [rBC]

Wick, K. R., Werner, E. D., Langlais, P., Ramos, F. J., Dong, L. Q., Shoelson, S. E. \& Liu, F. (2003) GRB10 inhibits insulin-stimulated insulin receptor substrate (IRS)-phosphatidylinositol 3-kinase/Akt signaling pathway by disrupting the association of IRS-1/IRS-2 with the insulin receptor. Journal of Biological Chemistry 278:8460-67. [aBC]
Wicks, S., Hjern, A., Gunnell, D., Lewis, G. \& Dalman, C. (2005) Social adversity in childhood and the risk of developing psychosis: A national cohort study. American Journal of Psychiatry 162:1652-57. [rBC]

Wiedl, K. H. (1992) Assessment of coping with schizophrenia. Stressors, appraisals, and coping behaviour. British Journal of Psychiatry Supplement 18:114-22. $[\mathrm{ST}]$

Wilkins, J. F. (2005) Genomic imprinting and methylation: Epigenetic canalization and conflict. Trends in Genetics 21:356-65. [aBC]

Wilkins, J. F. \& Haig, D. (2003) Inbreeding, maternal care, and genomic imprinting. Journal of Theoretical Biology 221:559-64. [DH]

(2003) What good is genomic imprinting: The function of parent-specific gene expression. Nature Reviews. Genetics 4(5):1-10. [GV]

Wilkinson, L. S., Davies, W. \& Isles, A. R. (2007) Genomic imprinting effects on brain development and function. Nature Reviews. Neuroscience 8(11):832-43. [WD]

Williams, C. A., Beaudet, A. L., Clayton-Smith, J., Knoll, J. H., Kyllerman, M., Laan, L. A., Magenis, R. E., Moncla, A., Schinzel, A. A., Summers, J. A. \& Wagstaff, J. (2006a) Angelman syndrome 2005: Updated consensus for diagnostic criteria. American Journal of Medical Genetics: Part A 140:413-18. [aBC]

Williams, D. (1992) Nobody nowhere: The extraordinary autobiography of an autistic. Doubleday Canada. [aBC]

Williams, D., Happé, F. \& Jarrold, C. (2008) Intact inner speech use in autism spectrum disorder: Evidence from a short-term memory task. Journal of Child Psychology and Psychiatry 49:51-58. [WF]

Williams, D. L., Goldstein, G. \& Minshew, N. J. (2006b) The profile of memory function in children with autism. Neuropsychology 20:21-29. [aBC]

Williams, J. H., Whiten, A., Suddendorf, T. \& Perrett, D. I. (2001) Imitation, mirror neurons and autism. Neuroscience and Biobehavioral Reviews 25:287-95. $[\mathrm{aBC}]$

Williams, L. M. \& Barry, J. (2003) Do sex differences in emotionality mediate sex differences in traits of psychosis-proneness? Cognition and Emotion 17:747-58. $\quad[\mathrm{aBC}]$

Winslow, J. T. \& Insel, T. R. (2004) Neuroendocrine basis of social recognition. Current Opinion in Neurobiology 14(2):248-53. [LMG]

Wisniewski, A., Milde, K., Stupnicki, R. \& Szufladowicz-Wozniak, J. (2007) Weight deficit at birth and Turner's syndrome. Journal of Pediatric Endocrinology and Metabolism 20:607-13. [rBC]

Woo, T. W. \& Crowell, A. L. (2005) Targeting synapses and myelin in the prevention of schizophrenia. Schizophrenia Research 73:193-207. [aBC]

Woodruff, P. W., McManus, I. C. \& David, A. S. (1995) Meta-analysis of corpus callosum size in schizophrenia. Journal of Neurology, Neurosurgery and Psychiatry 58(4):457-61. [GV]

Woods, B. T., Ward, K. E. \& Johnson, E. H. (2005) Meta-analysis of the time-course of brain volume reduction in schizophrenia: Implications for pathogenesis and early treatment. Schizophrenia Research 73:221-28. [aBC]

Wright, P., Nimgaonkar, V. L., Donaldson, P. T. \& Murray, R. M. (2001) Schizophrenia and HLA: A review. Schizophrenia Research 47:1-12. [rBC]

Wu, S., Jia, M., Ruan, Y., Liu, J., Guo, Y., Shuang, M., Gong, X., Zhang, Y., Yang, X. \& Zhang, D. (2005) Positive association of the oxytocin receptor gene (OXTR) with autism in the Chinese Han population. Biological Psychiatry 58(1):74-77. [LMG

Xiong, N., Ji, C., Li, Y., He, Z., Bo, H. \& Zhao, Y. (in press) The physical status of children with autism in China. Research in Developmental Disabilities. $[\mathrm{rBC}]$

Yamasaki, K., Joh, K., Ohta, T., Masuzaki, H., Ishimaru, T., Mukai, T., Niikawa, N., Ogawa, M., Wagstaff, J. \& Kishino, T. (2003) Neurons but not glial cells show reciprocal imprinting of sense and antisense transcripts of Ube3a. Human Molecular Genetics 12:837-47. [aBC]

Yamasue, H., Abe, O., Suga, M., Yamada, H., Rogers, M. A., Aoki, S., Kato, N. \& Kasai, K. (in press) Sex-linked neuroanatomical basis of human altruistic cooperativeness. Cerebral Cortex. [rBC]

Yan, W. L., Guan, X. Y., Green, E. D., Nicolson, R., Yap, T. K., Zhang, J., Jacobsen, L. K., Krasnewich, D. M., Kumra, S., Lenane, M. C., Gochman, P., Damschroder-Williams, P. J., Esterling, L. E., Long, R. T., Martin, B. M., Sitransky, E., Rapoport, J. L. \& Ginns, E. I. (2000) Childhood-onset schizophrenia/ autistic disorder and $\mathrm{t}(1 ; 7)$ reciprocal translocation: Identification of a BAC contig spanning the translocation breakpoint al 7 q21. American Journal of Medical Genetics 96(6):749-53. [ST]

Yaralian, P. S., Raine, A., Lencz, T., Hooley, J. M., Bihrle, S. E., Mills, S. \& Ventura, J. (2000) Elevated levels of cognitive-perceptual deficits in individuals with a family history of schizophrenia spectrum disorders. Schizophrenia Research 46:57-63. [aBC]

Yobb, T. M., Somerville, M. J., Willatt, L., Firth, H. V., Harrison, K., MacKenzie, J., Gallo, N., Morrow, B. E., Shaffer, L. G., Babcock, M., Chernos, J., Bernier, F., Sprysak, K., Christiansen, J., Haase, S., Elyas, B., Lilley, M., Bamforth, S. \& McDermid, H. E. (2005) Microduplication and triplication of 22q11.2: A highly variable syndrome. American Journal of Human Genetics 76:865-76. $[\mathrm{rBC}]$ 
References/Crespi \& Badcock: Psychosis and autism as diametrical disorders of the social brain

Yokoi, F., Dang, M. T., Li, J. \& Li, Y. (2006) Myoclonus, motor deficits, alterations in emotional responses and monoamine metabolism in epsilon-sarcoglycan deficient mice. Journal of Biochemistry 140:141-46. [rBC]

Yolken, R. H. \& Torrey, E. F. (2008) Are some cases of psychosis caused by microbial agents? A review of the evidence. Molecular Psychiatry 13:470-79. [rBC]

Young, E. J., Lipina, T., Tam, E., Mandel, A., Clapcote, S. J., Bechard, A. R., Chambers, J., Mount, H. T., Fletcher, P. J., Roder, J. C. \& Osborne, L. R. (2008) Reduced fear and aggression and altered serotonin metabolism in Gtf2irdl-targeted mice. Genes, Brain and Behavior 7:224-34. [rBC]

Young, R. L., Ridding, M. C. \& Morrell, T. L. (2004) Switching skills on by turning off part of the brain. Neurocase 10:215-22. [aBC]

Yücel, M., Stuart, G. W., Maruff, P., Velakoulis, D., Crowe, S. F., Savage, G. \& Pantelis, C. (2001) Hemispheric and gender-related differences in the gross morphology of the anterior cingulate/paracingulate cortex in normal volunteers: An MRI morphometric study. Cerebral Cortex 11:17-25. [aBC]
Zaitchik, D. (1990) When representations conflict with reality: The preschooler's problem with false beliefs and "false" photographs. Cognition 35:41-68. $[\mathrm{rBC}]$

Zhao, X. H., Wang, P. J., Li, C. B., Hu, Z. H., Xi, Q., Wu, W. Y. \& Tang, X. W. (2007) Altered default mode network activity in patient with anxiety disorders: An fMRI study. European Journal of Radiology 63:373-78. [aBC]

Zilbovicius, M., Meresse, I., Chabane, N., Brunelle, F., Samson, Y. \& Boddaert, N. (2006) Autism, the superior temporal sulcus and social perception. Trends in Neurosciences 29:359-66. [aBC]

Zinkstok, J. \& van Amelsvoort, T. (2005) Neuropsychology, development, and cognition: A journal on normal and abnormal development in childhood and adolescence. Child Neuropsychology 11:21-37. [aBC]

Zinn, A. R., Roeltgen, D., Stefanatos, G., Ramos, P., Elder, F. F., Kushner, H., Kowal, K. \& Ross, J. L. (2007) A Turner syndrome neurocognitive phenotype maps to Xp22.3. Behavioral and Brain Functions 3:24. [rBC] 